\title{
Direct photon and light neutral meson production in hadron collisions at the LHC with ALICE
}

\author{
Dissertation \\ zur Erlangung des Doktorgrades \\ der Naturwissenschaften
}

\author{
vorgelegt beim Fachbereich Physik \\ der Johann Wolfgang Goethe-Universität \\ in Frankfurt am Main \\ von \\ Nicolas Schmidt \\ aus Starnberg
}

Frankfurt am Main 2020

(D 30) 
vom Fachbereich Physik der

Johann Wolfgang Goethe-Universität als Dissertation angenommen.

Dekan: Prof. Dr. Harald Appelshäuser

Gutachter: $\quad$ Prof. Dr. Henner Büsching

Prof. Dr. Harald Appelshäuser

Datum der Disputation: 


\section{Zusammenfassung}

Während den ersten Mikrosekunden nach dem Urknall glaubt man, dass unser Universum aus einer heißen, dichten und stark wechselwirkenden Materie bestanden haben soll, welche man das Quark-Gluonen-Plasma (QGP) nennt. In diesem Medium sind die elementaren Bausteine der Materie, die Quarks und die Gluonen, nicht mehr in Hadronen gebunden, sondern können sich stattdessen wie quasi-freie Teilchen verhalten. Für die ALICE Kollaboration an CERN's Large Hadron Collider (LHC) ist die Untersuchung dieses Mediums eines der Hauptziele. Um dieses Medium im Labor zu erzeugen, werden Protonen und Nukleonen auf nahezu Lichtgeschwindigkeit beschleunigt und anschließend zur Kollision gebracht. Dabei werden Schwerpunktsenergien von bis zu $\sqrt{s}=13 \mathrm{TeV}$ bei Proton-Proton (pp) Kollisionen und bis zu $\sqrt{s_{\mathrm{NN}}}=5.02 \mathrm{TeV}$ bei Blei-Blei $(\mathrm{Pb}-\mathrm{Pb})$ Kollisionen erreicht. Bei solchen hochenergetischen Kollisionen werden die kritischen Werte der Energiedichte und Temperatur von jeweils $\mu \approx 1 \mathrm{GeV} / c$ und $T_{c} \approx 155 \mathrm{MeV}$ überschritten, welche mithilfe von "lattice QCD" bestimmt wurden. Sie bieten daher die perfekten Voraussetzungen für einen Phasenübergang von normaler Materie zu einem QGP. Die Entwicklung eines solchen Mediums, beginnend bei der eigentlichen Kollision, gefolgt von der Ausbildung des Plasmas und der letztendlichen Hadronisierung, kann jedoch nicht direkt untersucht werden, da das Plasma eine extrem kurze Lebensdauer hat. Die Studien die das QGP untersuchen möchten, müssen sich deshalb auf Teilchenmessungen und deren Veränderung aufgrund von Einflüssen durch das Medium beschränken. Es ist noch nicht definitiv geklärt, ob sich ein QGP nur in Kollisionen schwerer Ionen bildet, oder ob dies auch in kleineren Kollisionssystemen wie Proton-Proton oder Proton-Blei der Fall ist.

Damit in dieser Thesis Einschränkungen bezüglich einer möglichen Erzeugung eines mini-GQP in kleinen Kollisionssystemen gemacht werden kann, wird der Fokus auf Messungen von neutralen Pionen und $\eta$ Mesonen mit dem ALICE Detektor am CERN LHC gesetzt. Hierfür wird in einem Referenzsystem von Proton-Proton Kollisionen bei $\sqrt{s}=8 \mathrm{TeV}$ und in einem Proton-Blei $(\mathrm{p}-\mathrm{Pb})$ System bei $\sqrt{s_{\mathrm{NN}}}=8.16 \mathrm{TeV}$, welches eine nukleare Modifikation erfährt, gemessen und die Ergebnisse verglichen. Da in Proton-Proton Kollisionen die Bildung eines QGP, aufgrund zu geringer Energiedichte, nicht erwartet wird, dient eine Messung in diesem System als Messbasis, um Effekte der Kollision selbst von Effekten nach der Kollision zu separieren, welche die Teilchenproduktion beeinflussen. Teilchen können zusätzlich zu dem QGP auch mit kalter Kernmaterie interagieren, was sich in asymmetrischen Proton-Blei Kollisionen testen lässt. In diesem Kollisionssystem wird größtenfalls ein vergleichsweise kleines QGP gebildet, wohingegen das Blei Ion selbst als kalte Kernmaterie agieren kann. Zusätzlich zu den Mesonenmessungen wird in dieser Thesis auch die Erzeugung von direkten Photonen bei niedrigen Transversalimpulsen $\left(p_{\mathrm{T}}\right)$ in multiplizitätsabhängigen $\mathrm{p}-\mathrm{Pb}$ Kollisionen bei einer Schwerpunktsenergie von $\sqrt{s_{\mathrm{NN}}}=5.02$ $\mathrm{TeV}$ gemessen, welche als direkte Probe, sowie als charakteristisches Signal des QGP gilt.

Die neutralen Pionen, welche in dieser Thesis gemessen werden, kann man als einen Überlagerungszustand der zwei leichtesten Quarksorten, dem "up" (u) und dem "down" (d) Quark, sowie deren entsprechenden Anti-Teilchen $(\bar{u}$ und $\bar{d})$ verstehen. Das $\eta$ meson hingegen hat einen zusätzlichen Anteil des "strange" ( $s$ und $\bar{s}$ ) Quarks und eine resultierende höhere Masse. Quarks sind Teil des Standardmodells der Teilchenphysik, welches die Elementarteilchen und die zwischen ihnen wirkenden Elementarkräfte, ausgeübt durch Bosonen, beschreibt. Das Modell umfasst insgesamt sechs Quarks, welche sich durch ihre Masse und Ladung unterscheiden und als Grundbestandteil von gebundenen Zuständen, sogenannten Hadronen, fungieren. Die "up" und "down" Quarks gelten hierbei als die leichtesten Quarks und kommen daher am häufigsten in der Natur vor. Das bekannteste Beipiel stellen hier die allgemein bekannten Protonen (uud) und 
Neutronen $(u d d)$ dar, welche die Grundkomponenten von Nukleonen sind. Die restlichen Quarks tragen eine deutlich höhere Masse und haben daher eine große Tendenz, sich in leichtere Quarks umzuwandeln, wodurch ihre Lebensdauer sehr gering ist. Die "top" und "bottom" Quarks, welche die Schwersten sind, können daher nicht in gewöhnlicher Materie gefunden werden. Sie können jedoch experimentell durch hoch energetische Teilchenkollisionen erzeugt werden und indirekt über ihre Zerfallsprodukte nachgewiesen werden. Quarks tragen eine elektrische Ladung von entweder $\pm 1 / 3$ oder $\pm 2 / 3$, sowie eine Farbladung, wobei Letztere verantwortlich für ihre Bindung in Hadronen ist. Hadronen bestehen entweder aus drei Quarks, dann werden sie Baryonen genannt, oder aus einem Quark-Antiquark Paar, welches Meson genannt wird. Diese gebundenen Zustände erfüllen eine insgesamt neutrale Farbladung, sowie eine vollzählige elektrische Ladung. Des Weiteren gibt es auch exotische Penta-Quark Zustände, welche aus vier Quarks und einem Antiquark bestehen und bereits experimentell nachgewiesen wurden. Aufgrund der starken Wechselwirkung, welche durch Gluonen vermittelt wird, können Quarks nicht einzeln beobachtet werden. Das Potenzial der starken Kraft wird durch einen Coulomb Term, welcher mit kleineren Abständen größer wird, und einen zweiten Term, welcher das String-Potential beschreibt und für große Abstände zunimmt. Durch den zweiten Term ist es daher möglich, dass bei genügend großen Abständen sich ein neues Quark-Antiquark Paar bildet, wenn die Energie ausreichend ist. Der Coulomb Term hängt zudem von der Kopplungskonstante $\alpha_{s}$ ab, welche für große Impulsüberträge $Q^{2}$ schwindet und daher auch laufende Kopplungskonstante genannt wird. In einem QGP sind die Energiedichte und Temperatur daher groß genug, um das Bindungspotential der Quarks zu überwinden, wodurch sich die Teilchen mit Farbladung wie quasi-freie Teilchen bewegen können.

Am CERN LHC werden die notwendigen hohen Energien für die Erzeugung eines QGP durch Kollisionen von Nukleonen mit nahezu Lichtgeschwindigkeit erreicht. Dies setzt eine lange Kette an Vorbeschleunigern voraus, welche mit Wasserstoff und Blei Atomen beginnt. Die Atomen werden von ihren Elektronen befreit und danach in Linear- und Kreisbeschleuniger eingespeist welche sie letztendlich in den LHC leiten. Im LHC werden die Teilchen auf entgegengesetzten Bahnen beschleunigt und an vier vorbestimmten Stellen, an denen sich die großen Experimente ALICE, ATLAS, CMS und LHCb befinden, zur Kollision gebracht. Jedes dieser Experimente ist mit einer Vielzahl an Detektoren ausgestattet, um ein individuelles Bild der Teilchen zu erstellen, welche bei den Kollisionen erzeugt werden. Da die meisten dieser Teilchen sehr kurzlebig sind und daher direkt in andere Produkte zerfallen, müssen die Detektoren in der Lage sein, die resultierenden neutralen und geladenen Teilchen $\mathrm{zu}$ verfolgen und $\mathrm{zu}$ identifizieren. Das ATLAS und das CMS Experiment sind als Vielzweckdetektoren gebaut worden, welche ihren Forschungsschwerpunkt auf die Messung des Higgs Bosons, sowie Physik außerhalb des Standardmodells gelegt haben. Bei den Detektoren dieser Experimente wurde daher besonders Fokus darauf gesetzt, dass sie hohe Luminositäten inspizieren können, um somit die Anzahl an Kollisionsereignissen (Events) zu maximieren, welche diese seltenen Prozesse mit geringem Wirkungsquerschnitt enthalten. Die Detektoren sind daher so gestaltet, dass sie hohe Luminositäten vertragen können, um die Aufzeichnung von seltenen und interessanten Events zu maximieren. Das LHCb Experiment folgt einem anderen Prinzip und wurde entworfen, um bei hohen Pseudrorapiditäten nach seltenen charm und beauty Zerfällen zu suchen, sowie Paritätsverletzungen zu untersuchen. Der ALICE Detektor, dessen aufgezeichneten Daten in dieser Thesis verwendet werden, ist das einzige Experiment am LHC, welches speziell für SchwerionenKollisionen vorgesehen ist. Der Detektor ist in der Lage tausende Teilchen pro Einheitsrapidität $\mathrm{zu}$ verfolgen und gleichzeitig zu identifizieren, was in zentralen $\mathrm{Pb}-\mathrm{Pb}$ Kollisionen notwendig ist. Grundsätzlich ist der ALICE Detektor in zwei Teile separierbar. Einerseits in den vorwärts gerichteten Myonenarm und andererseits in den sogenannten "central barrel", welcher den Interaktionspunkt mit zylindrischen Detektorsystemen umschließt und von einem großen roten 
Solenoidmagneten eingefasst ist, der ein homogenes magnetisches Feld von bis zu 0.5 T Feldstärke generieren kann. Die Analysen in dieser Thesis verwenden ausschließlich Detektoren aus dem "central barrel". Am nächsten zum Interaktionspunkt ist das Inner Tracking System (ITS) angeordnet, welches ein zylindrisches Siliziumdetektorsystem ist, das aus jeweils zwei Lagen SiliziumPixel-, Silizium-Streifen- und Silizium-Driftdetektoren aufgebaut ist. Zusammen mit der Time Projection Chamber (TPC), einer massiven Zeit-Projektionskammer, werden diese Systeme zur Spurrekonstruktion und Teilchenidenfikation bis zu niedrigen Transversalimpulsen verwenden, sowie für die Bestimmung des primären Interaktionspunktes. Die Spurrekonstruktion geladener Teilchen ist mit der TPC möglich, da diese Teilchen das Gas beim Durchqueren ionisieren und die resultierenden Elektronen und geladenen Ionen durch ein angelegtes elektrisches Feld entweder zur zentralen Anode driften oder zu den Endplatten, welche die Ausleseelektronik beherbergen. Je nachdem, wie stark das Gas ionisiert wird, lässt sich daraus schliessen, um was für ein Teilchen es sich handelt, da dessen Energieverlust $(\mathrm{d} E / \mathrm{d} x)$ mit dem erwarteten Energieverlust korreliert werden kann. Dies verlangt jedoch, dass die Detektoren besonders sorgfältig kalibriert sind, um die unbeständigen Bedingungen während der Datenaufzeichnung auszugleichen. Für geladene Teilchen kann zudem aus der rekonstruierten Spur der Transversalimpuls aus der Krümmung durch das magnetische Feld bestimmt werden, wobei die Genauigkeit der Bestimmung von der Anzahl der verfügbaren Spurpunkte abhängt. Weiter außen gelegen in radialer Richtung befindet sich der Transition Radiation Detektor (TRD), ein Übergangsstrahlungsdetektor, welcher in den vorliegenden Analysen ausschließlich zur Verbesserung der TPC Spurrekonstruktion verwendet wurde. Der Detektor selbst kann auch dazu verwendet werden, um Elektronen und Pion voneinander zu unterscheiden, sowie zur Auslösung der Eventaufzeichung, ein sogenannter Trigger, welcher bevorzugt Events mit interessanten Elektronensignalen betrachtet. Direkt anschließend an den TRD befindet sich der Time of Flight (TOF) Detektor, welcher in ALICE als Zeitgeber verwendet wird und des weiteren Teilchenidentifikation bei höheren Impulsen liefern kann.

Ein Großteil der Ergebnisse in dieser Thesis bei hohen Transversalimpulsen basiert auf dem elektromagnetischen Kalorimeter (EMCal/DCal), sowie dem Photon Spectrometer (PHOS). Diese Kalorimeter befinden sich mehr als vier Meter vom Interaktionspunkt und umschließen nicht den vollständigen Raumwinkel. Das EMCal deckt jedoch im Vergleich zu PHOS ungefähr eine zehnfache Akzeptanz ab. Die Kalorimeter erlauben zudem eine Verbesserung der Statistik bei hohen Transversalimpulsen durch speziell entwickelte Event Trigger, welche auf hohen deponierten Energien basieren und mit Photonsignalen assoziiert sind. Die Rekonstruktion von Teilchen mit den elektromagnetischen Kalorimetern basiert auf deren deponierter Energie, welche meistens über mehrere Zellen verteilt wird. Daher wird ein Algorithmus angewendet, welcher Zellen mit einem Signal in Cluster zusammenfasst ("clusterized"), sodass die gesamte Teilchenenergie in einem rekonstruierten Objekt erfasst ist. Hierfür müssen verschiedene kritische Kalibrierungen auf Zellen- und Clusterebene angewendet werden, welche unterschiedliche Detektoreffekte ausgleichen und dafür sorgen, dass Daten und Simulationen übereinstimmen. Eine der wichtigsten dahingehenden Korrekturen ist für die Nonlinearität der Detektor Hardware, wodurch überproportional geringe Energien bei hohen Energiedeponierungen rekonstruiert werden. Diese Korrekturen wurden durch detailierte Studien an EMCal Testmodulen mit verschiedenen Strahlenergien des Proton Synchrotron und Super Proton Synchrotron am CERN bestimmt. Zusätzlich wurden EMCal Module im Labor darauf getestet, wie sie auf eingehende Signale reagieren, wodurch eine signifikante Nonlinearität bestimmt wurde. Diese Studien haben notwendige Erkenntnisse gewährt, welche den Vorstoß in die hochenergetischen Regionen mit EMCal erlauben.

Ein Ziel dieser Thesis ist zu bestimmen, inwiefern sich die Teilchenausbeute von neutralen Pionen und $\eta$ Mesonen im p-Pb Kollisionssystem im Vergleich zu einem pp Referenzsystem bei der 
gleichen Schwerpunktsenergie verändert. Die neutralen Mesonen werden hierfür in ihrem zwei Photon Zerfallskanal rekonstruiert, welcher dominant für das $\pi^{0}(\mathrm{BR} \approx 98.9 \%)$ und das $\eta$ Meson $(\mathrm{BR} \approx 39.4 \%)$ ist. Es wird erwartet, dass die Teilchenproduktion durch die Gegenwart des Blei Nukleus beeinflusst wird im Vergleich zu reinen pp Kollisionen. Diese Modifikationen lassen sich zum Großteil auf Mehrfachstreuung, Gluonsaturation und Energieverlust in kalter und heißer Materie zurückführen. Ein Teil davon lässt sich theoretisch durch nukleare Partonverteilungsfunktionen (nPDF) beschreiben, welche die Partondichte im Hadron bei einem gegebenen Impulsanteil $x$ angeben und den Einfluss des Nukleus absorbieren können. Die in dieser Thesis präsentierten Ergebnisse können dazu dienen, die Unsicherheiten verschiedener nPDFs weiter einzuschränken, da die Messungen einen großen Bereich von $x$ und Impulsübertrag $Q^{2}$ abdecken. Des Weiteren lassen sich durch die neuen Ergebnisse bei hohen Transversalimpulsen weitere Einschränkungen bezüglich des Energieverlustes in kalter und heißer Materie machen, wobei letzteres in kleinen Kollisionssystemen ( $\mathrm{pp}$ und $\mathrm{p}-\mathrm{Pb}$ ) als vernachlässigbar gilt. Weitere Untersuchungen bezüglich einer Ausbildung eines QGP in $\mathrm{p}-\mathrm{Pb}$ Kollisionen werden zudem durchgeführt, indem die Produktion direkter Photonen bei niedrigen Transversalimpulsen und hoher Multiplizität mit dem Überschussverhältnis $R_{\gamma}$ bestimmt wird. Als direkte Photonen werden alle Photonen bezeichnet, die nicht aus Teilchenzerfällen kommen und bei niedrigen Teilchenimpulsen können sie ähnlich wie Schwarzkörperstrahlung vom heißen QGP abgestrahlt werden. Sollte kein Signal bei niedrigem $p_{\mathrm{T}}$ aufzufinden sein, dann könnte dies als Beweis gelten, dass sich in $\mathrm{p}-\mathrm{Pb}$ Kollisionen kein signifikantes QGP bildet. Bei hohem $p_{\mathrm{T}}$ werden in jedem Kollisionssystem signfikante Signale prompter Photonen erwartet, welche unabhängig vom QGP produziert werden, und sich durch NLO pQCD Berechnungen beschreiben lassen, sowie im selben Zuge diese Berechnungen weiter einschränken können.

Die Proton-Proton Daten, die in dieser Thesis verwendet werden, wurden bei einer Schwerpunktsenergie von $\sqrt{s}=8 \mathrm{TeV}$ im Jahr 2012 aufgezeichnet. Die Datennahmen in diesem Jahr folgten vorhergehenden Detektorakzeptanzerhöhungen, welche kritisch fuer die Ergebnisse dieser Thesis bei hohem Transversalimpuls sind. Insgesamt wird eine integrierte Luminosität von $\mathscr{L}=650 \mathrm{nb}^{-1}$ betrachtet, wobei die Daten mit Minimum-Bias- und EMCal-Triggern aufgezeichnet wurde. Die p-Pb Daten für die neutrale Mesonenanalyse wurden im Jahr 2016 erfasst bei einer Schwerpunktsenergie von $\sqrt{s_{\mathrm{NN}}}=8.16 \mathrm{TeV}$ und beinhalten auch Minimum-Bias- und EMCal-Triggerdaten mit einer integrierten Luminosität von $\mathscr{L}=46 \mathrm{nb}^{-1}$. Für die Analyse der direkten Photonen werden p-Pb Daten bei $\sqrt{s_{\mathrm{NN}}}=5.02 \mathrm{TeV}$ verwendet, welche im Jahr 2013 aufgezeichnet wurden und aus einer großen Menge an Minimum-Bias-Events bestehen, wodurch eine gute Präzision bei niedrigen Transversalimpulsen ermöglicht wird. Photonen werden in der Analyse entweder aus $e^{+} e^{-}$Paaren rekonstruiert, welche durch Photonkonversionen im Detektormaterial erzeugt werden, oder aus geclusterten deponierten Energien in den Kalorimetern. Die Methode basierend auf Photonkonversionen hängt stark vom verfügbaren Material im inneren Teil des "central barrel" ab, welches sich zu einer Strahlungslänge von $0.1 X / X_{0}$ summiert, was einer Konversionswahrscheinlichkeit von ungefähr $9 \%$ entspricht. Im Vergleich dazu können Photonen mit den elektromagnetischen Kalorimetern mit einer deutlich höheren Effizienz rekonstruiert werden, welche aber eine schlechtere Energieaufösung haben, da ungefähr $50 \%$ aller Photonen im äußeren Detektormaterial konvertieren und zusätzlich geladene Teilchen minimal ionisierenden Energieverlust im EMCal hinterlassen. Neutrale Mesonen werden aus jeweils zwei Photonen über ihre invariante Masse rekonstruiert, wobei beide Photonen entweder von der selben oder aus unterschiedlichen Rekonstruktionsmethoden stammen. Die invariante Massenverteilung zeigt dann einen deutlichen Überschuss in der Region um die eigentliche $\pi^{0}$ und $\eta$ Meson Masse angesiedelt auf einem kombinatorischen Hintergrund. Dieser Hintergrund wird beschrieben, indem Photonen aus unterschiedlichen Events gemischt werden, da dies auss- 
chließlich die kombinatorische und akzeptanzgetriebenen Effekte beschreibt. Eine zweite Methode, um neutrale Pionen zu messen, wird bei hohen Transversalimpulsen angewendet, da dort die einzelnen Zerfallsphotonen nicht mehr separat in der Granularität des EMCal rekonstruiert werden können. Die überlappenden elektromagnetischen Schauer beider Photonen werden stattdessen in der "merged" Clusteranalyse verwendet, da sie als ein zusammenhängender Schauer mit vorwiegend elliptischer Form rekonstruiert werden. Alle beschriebenen Rekonstruktionsmethoden benötigen verschiedene Korrekturfaktoren, um von unkorrigierten Teilchenausbeuten zu voll korrigierten differentiellen invarianten Ausbeuten zu gelangen. Diese Korrekturen werden mit Monte Carlo Simulationen bestimmt, welche eine vollständige Detektorsimulation durchlaufen haben. Zusätzlich werden Datengestütze Ansätze verwendet, um die Reinheit ("purity") der Kandidaten zu bestimmen.

Mithilfe der beschriebenen Rekonstruktionsmethoden ist es möglich, den $p_{\mathrm{T}^{\mathrm{T}}}$-differenziellen invarianten Wirkungsquerschnitt der $\pi^{0}$ Meson in einer Impulsregion von $0.3(0.4)<p_{\mathrm{T}}<200$ $\mathrm{GeV} / c$ und den der $\eta$ Mesonen in $0.5(1.0)<p_{\mathrm{T}}<35(50) \mathrm{GeV} / c$ in pp $(\mathrm{p}-\mathrm{Pb})$ Kollisionen zu bestimmen. Diese Messungen gelten als die bisher höchsten Transversalimpulsmessungen identifizierter Teilchensorten und übertreffen vorherige Messungen von ALICE um nahezu eine Größenordnung. Die systematischen Unsicherheiten auf den Spektren werden anhand von Variationen der Auswahlkriterien in der Analyse bestimmt und belaufen sich auf 4-15\% für das $\pi^{0}$ und 6-15\% für das $\eta$. Die Unsicherheiten stammen größtenteils von der Signalextraktion, der Unsicherheit über das Detektor Material und der Energieauflösung der EMCal Cluster, wobei die statistischen Unsicherheiten ungefähr einen Faktor 2-3 kleiner sind als ihr systematisches Gegenstück. Die individuellen Mesonspektren werden von NLO pQCD Berechnungen, basierend auf aktuellen nPDFs und Fragmentationsfunktionen (FF), signifikant überschätzt, wohingegen Pyтнia8 Vorhersagen die Spektren generell unterschätzen. Aus den Spektren wird zudem das $\eta / \pi^{0}$ Verhältnis bestimmt, welches sich auf einen übereinstimmenden Wert bei hohem $p_{\mathrm{T}}$ in beiden Kollisionssystemen mit $C_{\mathrm{pp}}^{\eta / \pi^{0}} \approx 0.48$ beläuft. Die neuen $\eta / \pi^{0}$ Messungen werden zudem verwendet, um neue globale Fits in pp und p-A zusammen mit Messungen bei niedrigeren Kollisionsenergien zu bestimmen.

Der nukleare Modifikationsfaktor $R_{\mathrm{pA}}$ is des Weiteren aus dem skalierten Verhältnis aus den pp und $\mathrm{p}-\mathrm{Pb}$ Messungen bestimmt und zeigt sich übereinstimmend mit pQCD NLO Berechnungen, Color Glass Condensate (CGC) Vorhersagen, sowie Berechnungen von Energieverlust in kalter Materie (FCEL). Bei hohen Transversalimpulsen zeigt sich keine Modifikation der Teilchenproduktion, da $R_{\mathrm{pA}}$ mit Eins innerhalb der Unsicherheiten übereinstimmt. Dieses Ergebnis zeigt eine mögliche Spannung mit einer Messung geladener Hadronen von CMS, in welcher eine leicht erhöhte Teilchenproduktion in $\mathrm{p}-\mathrm{Pb}$ bestimmt wurde bis zu $p_{\mathrm{T}} \approx 125 \mathrm{GeV} / c$. Die neue Messung zeigt zudem eine geringfügig stärkere Unterdrückung der Teilchenproduktion bei mittleren $p_{\mathrm{T}}$ Werten im Vergleich zu einer vorherigen ALICE Messung bei $\sqrt{s_{\mathrm{NN}}}=5.02 \mathrm{TeV}$. Zum Abschluss des Mesonenkapitels wird ein Ausblick auf multiplizitätsabhängige Messungen in p- $\mathrm{Pb}$ und $\mathrm{Pb}-\mathrm{Pb}$ bis zu sehr hohem Transversalimpuls gegeben, wodurch weitere Einschränkungen für theoretische Modelle zur Verfügung gestellt werden könnten.

Das letzte Kapitel befasst sich mit den Messungen von inklusiven und direkten Photonen als Funktion der Eventmultiplizität in p-Pb Kollisionen bei einer Schwerpunktsenergie von $\sqrt{s_{\mathrm{NN}}}=$ $5.02 \mathrm{TeV}$. Die differenziellen invarianten Photonausbeuten werden in einer Impulsregion von $0.4<$ $p_{\mathrm{T}}<30 \mathrm{GeV} / c$ extrahiert, wobei totale Unsicherheiten von $4-5 \%$ erreicht werden. Diese Ergebnisse, zusammen mit Zerfallssimulationen basierend auf Parametrierungen gemessener Spektren, werden verwendet, um das Überschussverhältnis $R_{\gamma}$ zu bestimmen. $R_{\gamma}$ zeigt keinen Überschuss direkter Photonen bei niedrigem $p_{\mathrm{T}}$ innerhalb der Unsicherheiten, wodurch sich kein Signal ther- 
maler Photonen als Nachweis eines QGP bestimmen lässt. Die Messung ist jedoch limitiert in ihrer Präzision durch die totalen Unsicherheiten von 5-10\%, welche keine Sensitivität auf die vorhergesagten 1-3\% an thermalen Photonen erlauben. Für zukünftige Messungen ist es daher kritisch, dass die statistischen, sowie systematischen Unsicherheiten reduziert werden. Bei hohen Transversalimpulsen und hohen Multiplizitäten zeigt sich hingegen ein Überschuss prompter Photonen, welcher mit NLO pQCD Vorhersagen übereinstimmt. Basierend auf den $R_{\gamma}$ Messungen lässt sich die differentielle invariante Ausbeute direkter Photonen für Datenpunkte, die einen Überschuss zeigen, bestimmen. In allen anderen Fällen werden obere Grenzen mit einem 90\% Konfidenzniveau bestimmt, welche mit den Vorhersagen theoretischer Modelle übereinstimmen. Zuletzt wird ein Ausblick auf mögliche Verbesserungen der Unsicherheiten gegeben, welche einen großen Minimum-Bias Datensatz aus dem Jahr 2016, sowie Studien hinsichtlich der Detektormaterialimplementation in Monte Carlo Simulationen umfassen. 


\section{Contents}

1 Introduction 1

2 Physics Effects and Underlying Theory 3

2.1 Standard Model and QCD . . . . . . . . . . . . . . . . . . 3

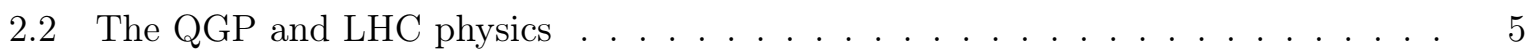

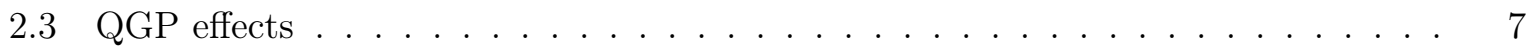

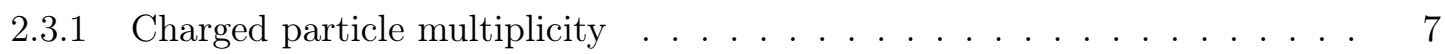

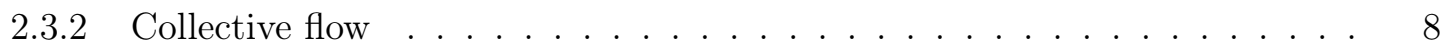

2.3.3 Modification of particle production . . . . . . . . . . . . . . 9

2.3.3.1 Cronin effect and multiple scattering . . . . . . . . . . 10

2.3.3.2 Nuclear parton distribution functions . . . . . . . . . . . . . 10

2.3.3.3 Energy loss in cold and hot nuclear matter . . . . . . . . . . . 11

2.3.4 Electromagnetic probes . . . . . . . . . . . . . . . . . 12

3 Experimental Overview $\quad 17$

3.1 The Large Hadron Collider . . . . . . . . . . . . . . . . . . . . . . . . . 17

3.1 .1 Proton and ion source . . . . . . . . . . . . . . . 18

3.1 .2 Injection scheme . . . . . . . . . . . . . . . . . . . . . . . 19

3.1.3 LHC experiments and physics . . . . . . . . . . . . . . . 20

3.2 A Large Ion Collider Experiment (ALICE) . . . . . . . . . . . . . . . . . . . 23

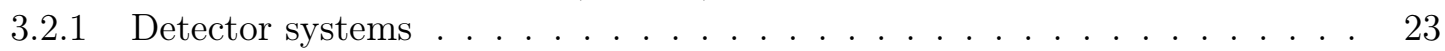

3.2.1.1 Inner Tracking System . . . . . . . . . . . . . . . . . 24

3.2.1.2 Time Projection Chamber . . . . . . . . . . . . . . . 25

3.2.1.3 Transition Radiation Detector . . . . . . . . . . . . 27

3.2.1.4 Time of Flight Detector . . . . . . . . . . . . . . 28

3.2.1.5 Electromagnetic Calorimeters EMCal and DCal . . . . . . . . 29

3.2.1.6 Photon Spectrometer PHOS . . . . . . . . . . . . . . . 31

3.2.1.7 High Momentum Particle Identification Detector (HMPID) . . . 32

3.2.1.8 Forward Detectors . . . . . . . . . . . . . . . . 32

3.2.2 ALICE software framework and reconstruction process . . . . . . . . . 33

4 Data sets $\quad 37$

4.1 Data samples . . . . . . . . . . . . . . . . . . . . . . 37

4.2 Event selection . . . . . . . . . . . . . . . . . . . 37

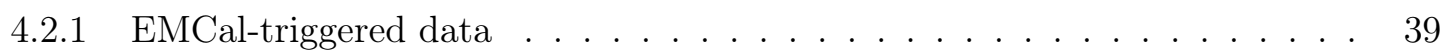

4.3 Monte Carlo simulations . . . . . . . . . . . . . . . . . . . . . 41

4.3.1 EMCal trigger simulation . . . . . . . . . . . . . . . 43

5 Photon Reconstruction $\quad 45$

5.1 Photon Conversion Method $(\mathrm{PCM}) \ldots \ldots \ldots \ldots \ldots$

5.2 EMCal photon clusters . . . . . . . . . . . . . . . . 50

5.2 .1 Cluster reconstruction . . . . . . . . . . . . . . . . 50

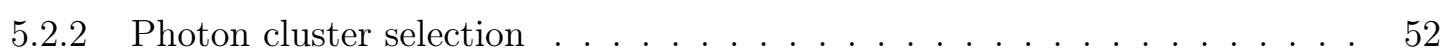

5.2.2.1 EMCal bad channel maps . . . . . . . . . . . . . . . . . 54

$5.2 .2 .2 \quad$ EMCal cell time calibration . . . . . . . . . . . . . . 54

5.2 .3 Energy nonlinearity and scale . . . . . . . . . . . . . . 55

5.2 .4 Cross-talk emulation . . . . . . . . . . . . . . . . . 57 
6 Meson Measurements $\quad 59$

6.1 Reconstruction via invariant mass . . . . . . . . . . . . . . . . . . . . 59

6.1 .1 Correction factors . . . . . . . . . . . . . . . . 64

6.1.1.1 Out-of-bunch pileup . . . . . . . . . . . . . . 6 64

6.1 .1 .2 Secondary neutral pions . . . . . . . . . . . . . . 66 66

6.1.1.3 Kinematic acceptance and reconstruction efficiency . . . . . . . 67

6.1 .2 EMCal triggered data . . . . . . . . . . . . . . . . . . . 69

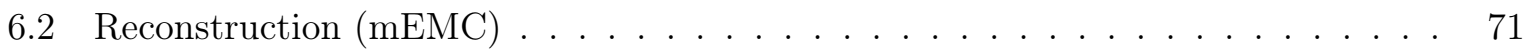

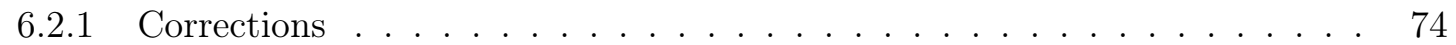

6.3 Systematic uncertainties . . . . . . . . . . . . . . . . . . 77

6.4 Correction for finite bin width . . . . . . . . . . . . . . . . . 82

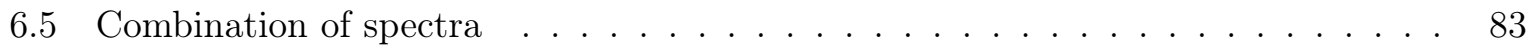

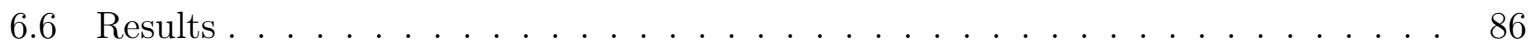

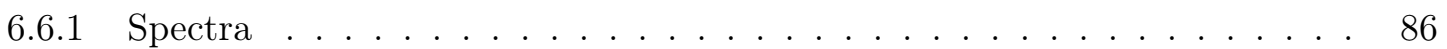

$6.6 .2 \eta / \pi^{0}$ ratio . . . . . . . . . . . . . . . . . . . . . . . 88

6.6 .3 Nuclear modification factor . . . . . . . . . . . . . . . . . . 90

6.7 Meson summary and outlook . . . . . . . . . . . . . . . . . 94

7 Direct Photon Measurements $\quad 97$

7.1 Cocktail simulation . . . . . . . . . . . . . . . . . . . 97

7.2 Multiplicity dependent inclusive and direct photon measurements . . . . . . . . 102

7.3 Systematic uncertainties . . . . . . . . . . . . . . . . . . . 107

7.4 Combination of measurements . . . . . . . . . . . . . . . . . . . . 110

7.5 Results . . . . . . . . . . . . . . . . . . . . . 114

7.6 Direct photon summary and outlook . . . . . . . . . . . . 117

$\begin{array}{llr}8 & \text { Summary } & 119\end{array}$

$\begin{array}{lr}\text { A Appendix } & 123\end{array}$

A.1 Overview of data samples and MC productions . . . . . . . . . . . . . 123

A.2 Example invariant mass distributions for $\mathrm{pp} \sqrt{s}=8 \mathrm{TeV} \ldots \ldots \ldots \ldots$

A.3 Invariant mass peak position and width in pp collisions . . . . . . . . . . . . . 124

A.4 Secondary neutral pion correction factors . . . . . . . . . . . 125

A.5 Correlations and weights for combination of triggers . . . . . . . . . 126

A.6 Systematic uncertainties . . . . . . . . . . . . . . . . . . . . . 128

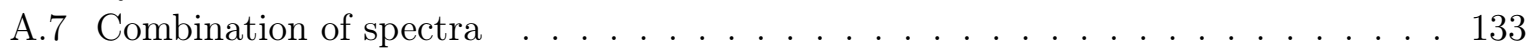

A.8 Nuclear modification factor . . . . . . . . . . . . . . . . . . . . . . . . 134

A.9 Direct and inclusive photons . . . . . . . . . . . . . . 135

A.9.1 Particle decay simulations . . . . . . . . . . . . . . . . . . . 135

A.9.2 Weights for combination . . . . . . . . . . . . . . 136

A.9.3 Relative uncertainties of combined spectra . . . . . . . . . . . . 137 


\section{Introduction}

The field of particle physics in on an ongoing quest to reach continuously higher precision measurements and to progress further into unexplored regimes. The main ingredient for these studies are high energetic particle collisions which lead to the creation of a multitude of predominantly unstable particles. At present, the Large Hadron Collider (LHC) at the European Organization for Nuclear Research (CERN) is the largest and most powerful particle accelerator allowing to collide protons at center-of-mass energies of up to $\sqrt{s}=14 \mathrm{TeV}$ and heavy-ions with a maximum of $\sqrt{s_{\mathrm{NN}}}=5.5 \mathrm{TeV}$ per nucleon pair. Such high energies provide ideal conditions in order to test theoretical models like the Standard Model as well as physics beyond the Standard Model. One of the main discoveries at the LHC was the measurement of the Higgs boson which was predicted in the 1960s as an additional constituent of the Standard Model. The focus of this thesis is on particle measurements with the ALICE experiment which is the dedicated heavyion experiment at the LHC and built to study a strongly coupled state of matter, called the quark-gluon plasma (QGP) which theoretical foundation and main signatures are discussed in Chapter 2. This medium, which should have existed shortly after the Big Bang, is expected to be produced in heavy-ion collisions and its presence in small collision systems, like pp and $\mathrm{p}-\mathrm{Pb}$, is still to be confirmed. Within the hot and dense QGP, quarks and gluons are no longer bound into hadrons and their unscreened color charges provide optimal circumstances to test the strong interaction. The QGP is probed either via measurements of physical observables that are affected by its presence, like jets and individual hadrons, or particles that are mostly unaffected, like direct photons. ALICE is capable of providing identified particle measurements up to the extreme charged-particle densities of central heavy-ion collisions while being able to cover a large transverse momentum range with its tracking and particle identification systems. The detector itself as well as the LHC accelerator complex are introduced in Chapter 3 which also provides additional information about the event reconstruction and analysis software framework.

In order to disentangle initial and final state effects observed in heavy-ion collisions a good understanding of small collision systems is necessary. This thesis therefore focuses on measurements of light neutral mesons, namely the neutral pion and eta meson, in $\mathrm{p}-\mathrm{Pb}$ collisions at $\sqrt{s_{\mathrm{NN}}}=8.16$ $\mathrm{TeV}$ with an additional reference measurement in pp collisions at a similar center-of-mass energy as well as on multiplicity dependent direct photons in $\mathrm{p}-\mathrm{Pb}$ collisions at $\sqrt{s_{\mathrm{NN}}}=5.02 \mathrm{TeV}$. The data samples as well as Monte Carlo simulations used to correct the data are introduced in Chapter 4. Both neutral mesons are reconstructed via their two-photon decay channel with up to five partially independent reconstruction techniques involving invariant mass-based signal extractions and a particle identification measurement focused on very high $p_{\mathrm{T}}$. The fundamentals of the photon reconstruction via the Photon Conversion Method (PCM) and the Electromagnetic Calorimeter (EMCal) are explained in Chapter 5 with further information on necessary detector calibrations. This is followed in Chapter 6 by detailed descriptions of the neutral meson reconstruction itself with the different reconstruction techniques in both collision systems as well as the corresponding systematic uncertainties. The chapter also outlines all necessary corrections, obtained either from Monte Carlo simulations or data-driven approaches, in order to obtain the fully corrected $p_{\mathrm{T}}$-differential invariant cross section spectra of both mesons. The measurement of multiplicity dependent direct photon production in $\mathrm{p}-\mathrm{Pb}$ collisions at $\sqrt{s_{\mathrm{NN}}}=5.02 \mathrm{TeV}$ is discussed in Chapter 7 with details on all necessary ingredients. The results of the meson and direct photon measurements are furthermore discussed with the goal to constrain cold nuclear matter effects and the possible creation of a QGP in such small systems. The thesis is subsequently wrapped up with a summary and an outlook to more differential studies. 


\section{Physics Effects and Underlying Theory}

This chapter provides an overview of the theoretical framework used to describe the results presented in this thesis. It briefly outlines the Standard Model of particle physics and the underlying field theories for the electromagnetic, weak and strong interaction in order to allow for a discussion of the heavy-ion experimental program and its key observables to study initial and final state effects on particle production.

\subsection{Standard Model and QCD}

With the discovery of the neutron as the last basic building block of atoms in 1932 by James Chadwick $[1,2]$ the nucleus could be described in its basic form. This discovery followed previous observations of electrons and protons in 1897 by J.J. Thomson [3] and in 1919 by Rutherford [4], respectively. Further investigations to improve on the definition of matter employed cyclotrons and cosmic rays via bubble chambers and lead to the discovery of muons in 1937 [5] and pions [6] and kaons [7] in the following decade. Shortly after, a variety of particle species with different combinations of spin, charge

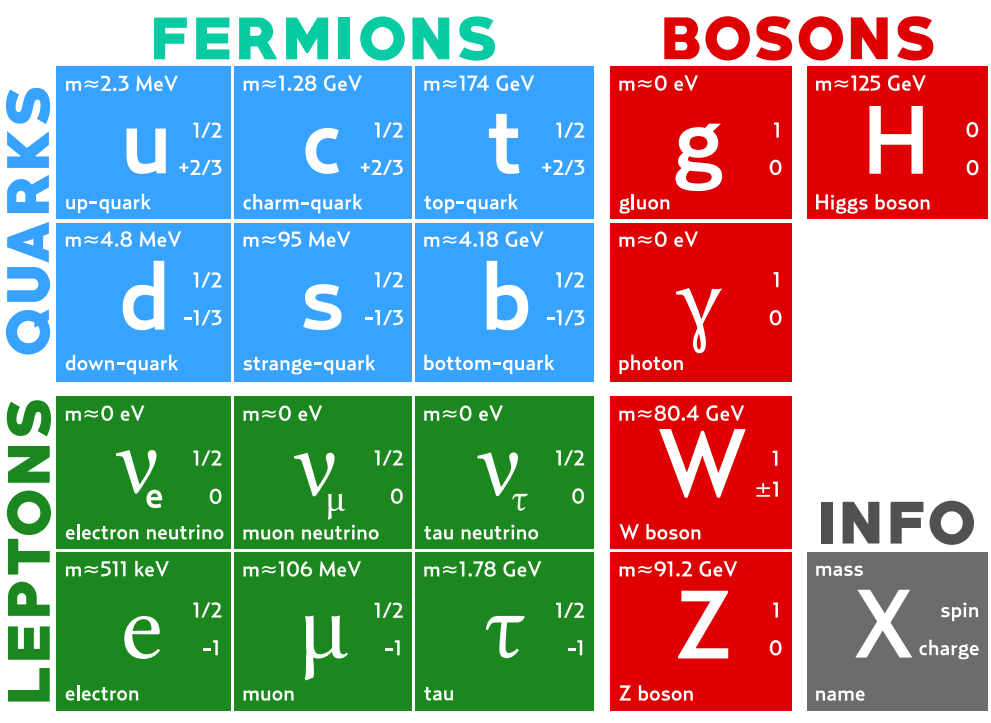

Figure 2.1: The Standard Model with its constituents and force carriers (fermions and bosons) as well as their mass, spin and charge.

or strangeness were discovered but did not fit into the existing model of protons, neutrons and electrons. This assortment of particles represented a challenge at the time called the "particle zoo" where it was not clear whether there was an order to the chaotic variety of different subatomic particles.

In 1962 Murray Gell-Mann organized these new particles according to their properties believing they must be made up of the same constituents and thus created triangular patterns referred to as the "eightfold way" based on strangeness, spin and charge [8]. From this, he determined a missing particle, the $\Omega^{-}$and therefore made a first prediction with his model. This prediction granted him a Nobel Price after the discovery of the particle in 1969. The triangular shape also hinted at three fundamental particles, later known as the up, down and strange quarks. With the addition of further quarks to this model, it was possible to explain even more particles and thus the Standard Model was formed [9-12]. The self-consistent Standard model, which is a Quantum Field Theory (QFT), is used to describe a variety of particles and their interactions via the electromagnetic, strong or weak force and is furthermore able to precisely explain a large fraction of experimental data [13].

With the addition of the Higgs boson prediction in 1964 by P. W. Higgs [14] and its subsequent discovery in 2012 by the CMS and ATLAS experiments at the CERN LHC [15,16], also the higgs mechanism could be described within this model [17].

A full overview of all fundamental particles and the force carriers of this model is shown in Figure 2.1. The fundamental particles are divided into two categories based on their spin; the half-integer spin fermions following Fermi-Dirac statistics and the integer spin bosons that 


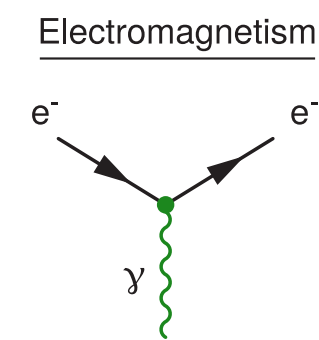

All charged particles Never changes flavour

$$
\alpha \approx 1 / 137
$$

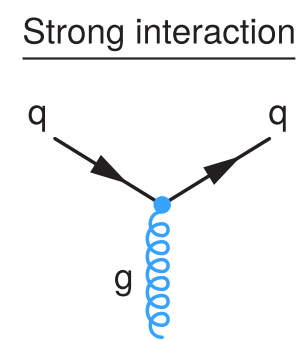

Only quarks

Never changes flavour

$$
\alpha_{S} \approx 1
$$

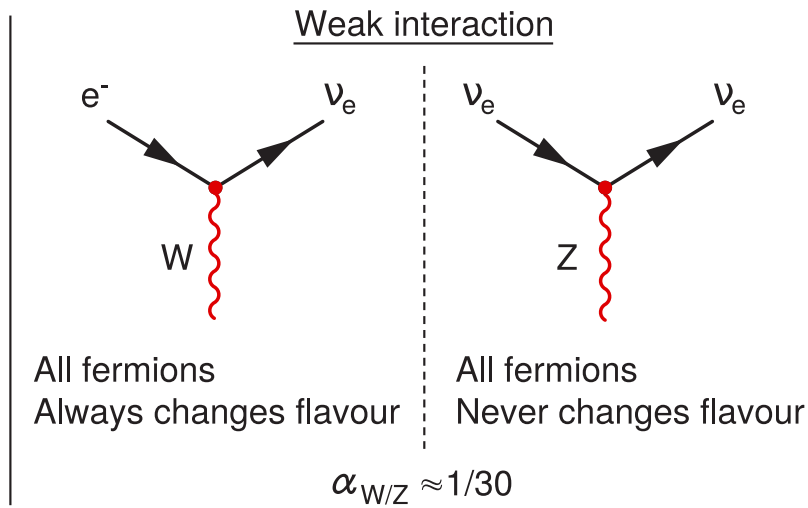

Figure 2.2: Feynman diagrams of the Standard Model interaction vertices for electromagnetic, weak and strong interactions together with their respective coupling constants $\alpha$. Adapted from Ref. [18].

follow Bose statistics. The gauge bosons mediate the three fundamental forces of the elementary interactions in the Standard Model as shown in Figure 2.2. These forces between the particles are described by Quantum Field Theories (QFT) and can be represented by Feynman diagrams. The electromagnetic interaction is described by Quantum Electrodynamics (QED) where the photon is the force carrying particle. This interaction is characterized by an infinite range and affects all electrically charged particles. Its electromagnetic coupling constant $(\alpha)$ is approximately $1 / 137$ allowing for precise calculations with perturbation theory. The weak interaction, which together with the electromagnetic interaction could be combined into the electro-weak theory, uses the $\mathrm{Z}^{0}$ and $\mathrm{W}^{ \pm}$bosons to mediate the force. As these bosons have a very high mass, this interaction only has a very limited range of $10^{-18} \mathrm{~m}$ and exclusively affects particles carrying weak hypercharge. The interaction furthermore allows for charge-parity $(\mathrm{CP})$ violation, breaking of parity symmetry and change of quark flavor, where the latter is given by the flavour quantum numbers (isospin, charm, strangeness, topness, bottomness) and consists of six flavors $(u, d, s, c, b, t)$. The underlying electro-weak theory additionally describes the Higgs mechanism which gives rise to the elementary particle masses in the Standard Model. The strong interaction is described by Quantum Chromodynamics (QCD) and its force is acting over small distances on the order of $10^{-15} \mathrm{~m}$ between particles that carry one of three color charges (red, green, blue) or their corresponding anti-colors. The three colors can be explained due to the degrees of freedom in the $\mathrm{SU}(3)$ gauge group on which QCD is based on. The force carriers are gluons which themselves carry a color charge and therefore can interact with each other. This self-coupling of the gluons results in an increase of the effective color charge leading to an anti-screening effect. The coupling strength of QCD $g_{s}$ is linked to the resulting running coupling constant $\alpha_{s}$ via $\alpha_{s}=g_{s}^{2} / 4 \pi$. This constant shows a strong dependence on the momentum transfer $Q^{2}$ which has to be largely de-

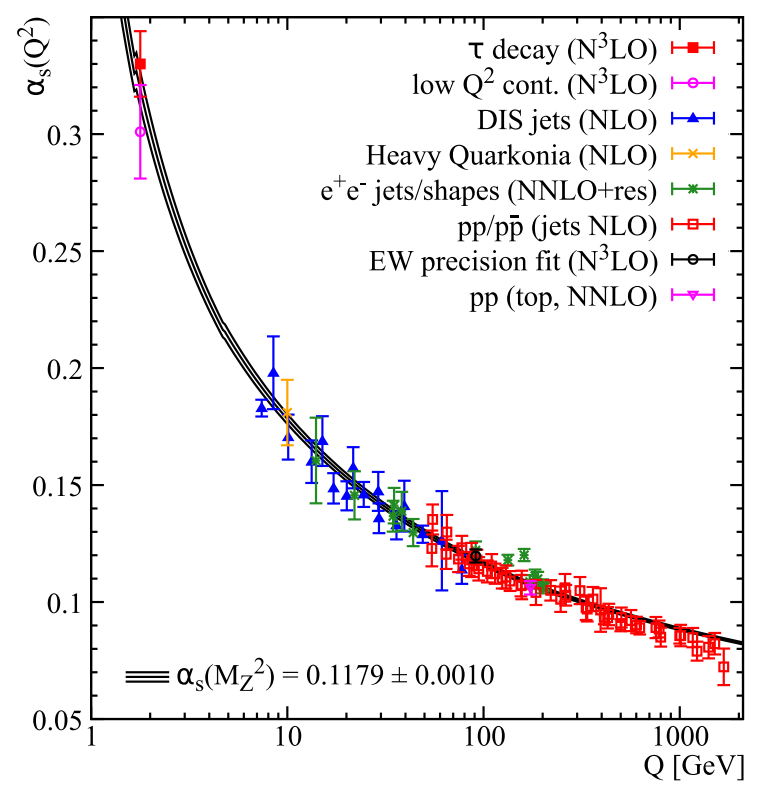

Figure 2.3: Energy scale $Q$ dependent measurements of $\alpha_{s}$ for different degrees of the QCD perturbation theory [19]. 
termined via experiments as it can only be partially constrained in the perturbative $\mathrm{QCD}$ regime. It is given to first order by

$$
\alpha_{s}\left(Q^{2}\right) \approx \frac{4 \pi}{\beta_{0} \ln \frac{Q^{2}}{\Lambda^{2}}}
$$

where the first order expansion of the QCD $\beta$ function is given by $\beta_{0}=\frac{11}{3} N_{c}-\frac{2}{3} N_{f}$ with $N_{c}$ and $N_{f}$ being the number of colors and active flavors $\left(m_{\text {quark }} \ll Q\right)$, respectively. The QCD scaling parameter is given by $\Lambda \approx 200 \mathrm{MeV}$ obtained from experiments. The dependence of $\alpha_{s}$ on $Q$ is shown in Figure 2.3 where a good agreement between the measured data and the pQCD theoretical prediction can be seen.

In the QCD theory two special properties are present; the socalled confinement and the asymptotic freedom depending on the

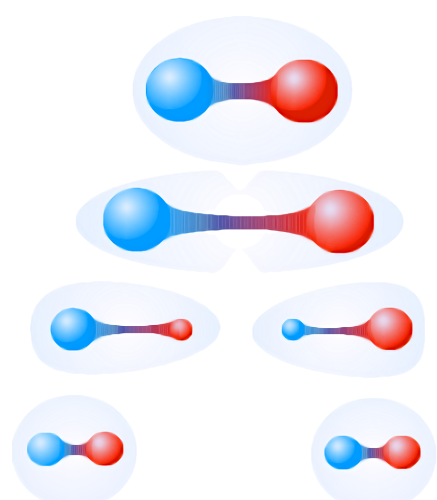

Figure 2.4: Confinement sketch with quark separation. Taken from Ref. [20]. momentum transfer region. The coupling constant perturbatively converges in the low momentum transfer region. This is interpreted as color confinement which is a property of color charge and states that quarks or gluons can not exist freely by themselves below the Hagedorn temperature of approximately $140 \mathrm{MeV}$ [21]. It is explained by the QCD potential of the strong interaction given by $V(r)=-\frac{4}{3} \frac{\alpha_{s}}{r}+\kappa r$, where the first term describes the asymptotic freedom like a Coulomb potential that becomes large for small distances $r$ and the second term describes the string potential with string tension $\kappa \approx 0.85 \mathrm{GeV} \mathrm{fm}^{-1}[22]$ which becomes large for large $r$. The consequence of this potential is that if two quarks would be pulled apart from each other, the energy density would increase and a new pair of quarks would be created as illustrated in Figure 2.4. We therefore only observe bound colorless quark states called hadrons which can be classified as mesons if they are made of a quark and anti-quark, or as baryons if they are made of three quarks, or exotic hadrons like tetra-quarks. In the region of large momentum transfer $Q \gg \Lambda$ the running coupling constant $\alpha_{s}$ is decreasing and therefore the coupling becomes weak. In the limit of $Q^{2} \rightarrow \infty$ the quarks and gluons are no longer coupled and can be treated as quasi-free particles. This feature is referred to as asymptotic freedom and allows the use of perturbative calculations.

\subsection{The QGP and LHC physics}

With the asymptotic freedom resulting from high energy densities it was deduced that a deconfined matter, called the QuarkGluon Plasma (QGP), can be formed which consists of free quarks and gluons instead of hadrons [23-25]. The conditions for such matter are expected to have existed until about 10 microseconds after the Big Bang. The two regions of the confined hadronic matter and the deconfined QGP can be seen in the QCD phase diagram shown in Figure 2.5 which represents the temperature $T$ versus the baryon chemical potential $\mu_{B}$. The regions are separated by a phase

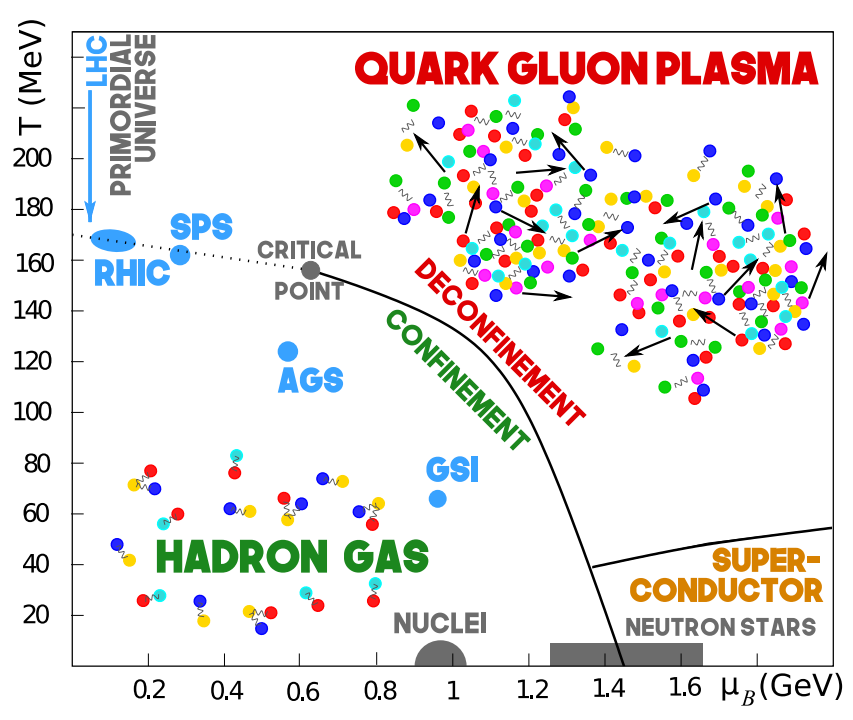

Figure 2.5: Schematic QCD phase diagram showing the temperature versus net baryon density. The critical point as well as the chemical freeze-out conditions of various experiments is indicated. 
transition which is smooth for vanishing baryon chemical potential $\left(\mu_{B}=0\right)$ up until a critical point where the transition turns into a first order phase transition. For high $\mu_{B}$ and low temperatures the transition is also expected. Such conditions are possible within neutron stars where the comparably low temperatures are accompanied by a large $\mu_{B}$ due to the gravitational collapse. In addition, another transition might be possible in this region into a hypothetical color superconducting phase.

The critical temperature $T_{c}$ at which hadronic matter undergoes its transition into the deconfined QGP has been estimated to be 100-250 MeV [26]. Furthermore, in the 1960s, Hagedorn discovered from his extensive matter studies that hadronic matter is limited to systems with temperatures of less than $140 \mathrm{MeV}$ [27].

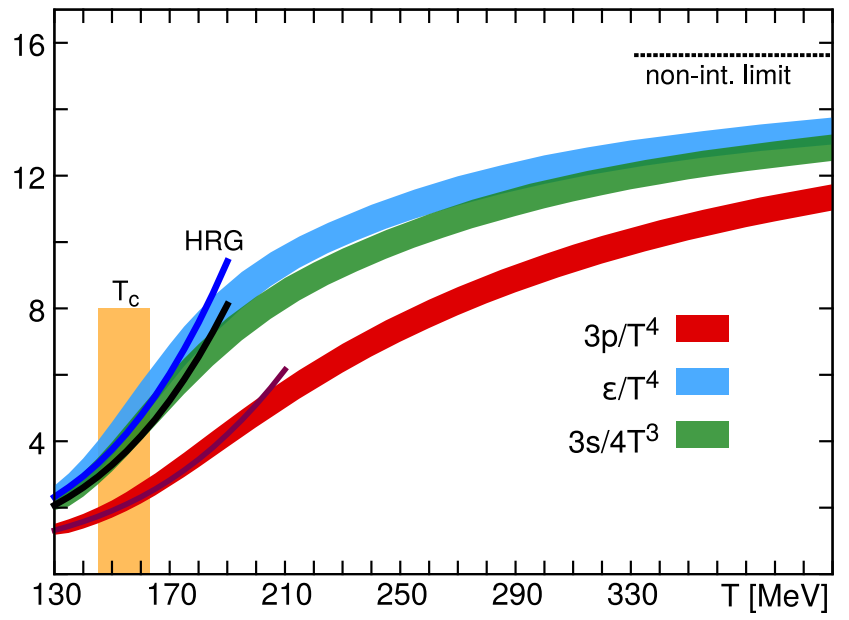

Figure 2.6: Temperature dependent normalized pressure, energy and entropy density compared to SHM model calculations [31]. The density limit for an ideal gas is indicated by the dotted line and the critical temperature region of $T_{c}=(154 \pm 9) \mathrm{MeV}$ is shown in orange. With the help of Lattice QCD (LQCD) calculations, which are non-perturbative calculations of OCD on a space-time lattice, $T_{c}$ could be determined in the low momentum transfer region leading to a more precise estimate of 150-170 $\mathrm{MeV}$ at $\mu_{B}=0[26,28]$. Such calculations also depend on the number of flavors that are used and thus can result in different critical temperatures [29,30]. When hadronic matter transitions to the deconfined phase, it splits up into its constituents of quarks and gluons leading to an increase of the degrees of freedom of the system. This effect can be seen in Figure 2.6 where a strong increase in the energy density is observed around the critical temperature. For higher temperatures, the medium does not gain additional degrees of freedom and instead only experiences increased heating leading to a flattening of the distributions. For sufficiently high enough temperatures, the system approaches the state of an ideal gas which is indicated in the same figure as the dotted line representing the Stephan Boltzmann limit.

In general, theoretical models of a QGP require the system to reach a state of local thermal equilibrium in order for their thermodynamic quantities to be defined $[32,33]$. This equilibrium state can be driven by large amounts of particles interacting with the medium itself, provided its lifetime is long enough. The conditions for a QGP are usually not fulfilled in small collision systems of particles like protons or leptons as such systems do not reach high enough particle densities. For larger collision systems, involving heavy-ions like lead or gold, it is very likely to produce a QGP state provided the collision energy is large enough. With such collisions it is possible to cover different areas of the phase diagram by using increasing center-of-mass energies which in turn lead to an increase of the temperature of the system and a resulting decrease in baryon density.

The exploration of relativistic heavy-ion collisions started in the early 1960s at the Alternating Gradient Synchrotron (AGS) experiment at the Brookhaven National Laboratory (BNL) [34] and the Super Proton Synchrotron (SPS) at CERN [35] with center-of-mass energies of $\sqrt{s_{\mathrm{NN}}}<20$ $\mathrm{GeV}$. The SPS accelerated protons as well as ${ }^{16} \mathrm{O}$ and ${ }^{28} \mathrm{Si}$ ions for its fixed target experiments where a first suppression of $\mathrm{J} / \Psi$ yield [36] depending on the centrality of the collision was observed. The centrality of a collision is related to the impact parameter $b$ which stands for the distance between the centers of both nuclei. Collisions with low values of $b$ are called "central" 
while for large values they are referred to as "peripheral". The relation between the impact parameter and the centrality of a collision can be described with the Glauber model [37]. The behavior of the centrality-dependent suppression was interpreted as the effect of the formation of a deconfined matter with a proper lifetime of $1.5 \mathrm{fm} \mathrm{[38]} \mathrm{for} \mathrm{which} \mathrm{the} \mathrm{energy} \mathrm{densities} \mathrm{at} \mathrm{the}$ SPS should suffice as shown in Figure 2.5. For further exploration of the QGP, the Relativistic Heavy Ion Collider (RHIC) was designed and built at BNL allowing for proton, deuteron, copper and gold ions to be accelerated up to $\sqrt{s}=510 \mathrm{GeV}$ in pp collisions and $\sqrt{s_{\mathrm{NN}}}=200 \mathrm{GeV}$ in $\mathrm{Au}+\mathrm{Au}$ collisions. Compared to the other accelerators, the LHC provides a thirtyfold increase in center-of-mass energy up to $\sqrt{s_{\mathrm{NN}}}=5.02 \mathrm{TeV}$ for $\mathrm{Pb}-\mathrm{Pb}$ collisions leading to temperatures much greater than the critical temperature and the possibility to study the QGP in great detail with dedicated experiments like the ALICE detector.

\subsection{QGP effects}

It is only via the final state quantities like the charged particle density, photon or lepton multiplicities, $p_{\mathrm{T}}$ spectra of various particles and elliptic flow measurements as direct observations of the medium that we can access the properties of the QGP, as it is so short lived. The mediums time evolution after the collision is responsible for different effects which are explained in the following.

\subsubsection{Charged particle multiplicity}

One of the most accessible observables for heavy-ion collisions is the charged particle multiplicity at mid-rapidity $\mathrm{d} N_{\mathrm{ch}} /\left.\mathrm{d} \eta\right|_{\eta=0}[40$, 41]. It is shown in Figure 2.7 versus center-ofmass energy for various experiments and collision systems. For a comparison between the collision systems, the dependence on the mean number of participant pairs is taken out. This $N_{\text {part }}$ normalization is chosen as particle production in heavy-ion collisions is expected to scale with the number of participating nucleons [42]. Furthermore, this scaling relation is dominantly assumed for soft processes which are responsible for the bulk of produced particles at low transverse momentum. From Figure 2.7 it can also be seen that measurements at high center-of-mass energies where necessary as with previous lower energies it was not possible to determine a reliable extrapolation to the LHC regime.

Aside from the center-of-mass energy dependence, there is also a centrality dependence of the particle production which can be described at RHIC and LHC energies with the same functional form with only a constant scaling factor accounting for the energy difference [43-45]. From this, it could be derived that at a given $N_{\text {part }}$ a similar production pro-

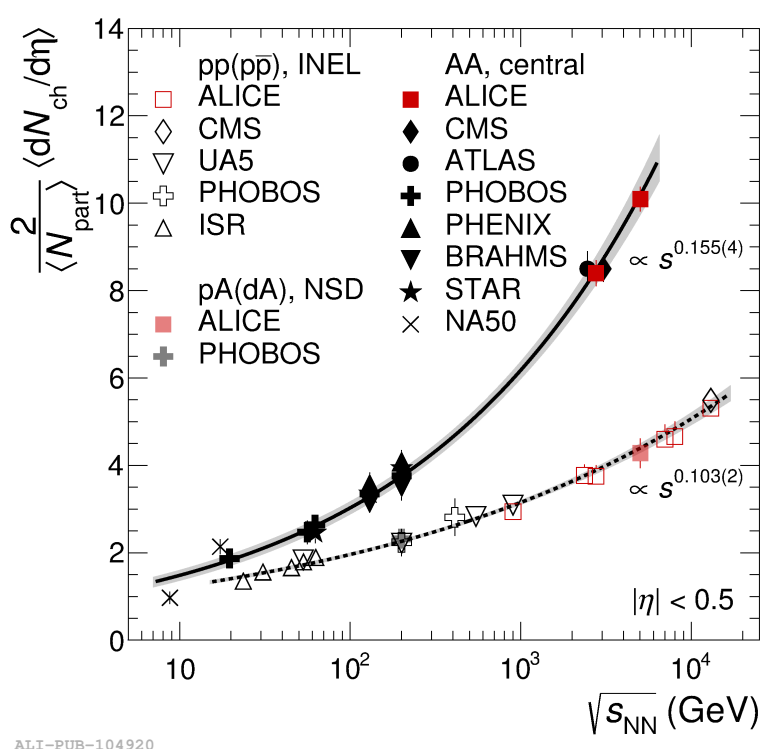

Figure 2.7: Dependence of the average charged particle density per unit pseudorapity versus $\sqrt{s_{\mathrm{NN}}}$ for various collision systems [39]. The small system $s$-dependence can be described by a power-law function with $s_{\mathrm{NN}}^{0.103}$ (dashed line) while heavy-ion collision data is described by $s_{\mathrm{NN}}^{0.155}$ (solid line) with the gray band indicating the uncertainty. 
cess is dominant independent of the center-of-mass energy and that an observed decrease of the charged particle density at lower centralities allows to probe a lower initial temperature of the system. Measurements as shown in Figure 2.7 provide a gauge for the energy density of the collision via the Bjorken estimate of the initial energy density $\epsilon_{B}[46]$ calculated as

$$
\epsilon_{B}=\left.\frac{\left\langle m_{\mathrm{T}}\right\rangle}{\tau \cdot A} \frac{\mathrm{d} N}{\mathrm{~d} \eta}\right|_{\eta=0}=\left.\frac{1}{\tau \cdot A} \frac{\mathrm{d} E_{\mathrm{T}}}{\mathrm{d} \eta}\right|_{\eta=0},
$$

with $A$ being the overlap region of the nuclei and $\tau$ the formation time of the system quanta which is in principle unknown but can be constrained for example from the formation time of quanta with an average transverse mass. If $\epsilon_{B}$ exceeds the critical value of $1 \mathrm{GeV} / \mathrm{fm}^{3}$ as predicted via lattice QCD calculations, the condition for the formation of a QGP is fulfilled [47]. From the measured values of $\mathrm{d} N_{\mathrm{ch}} / \mathrm{d} \eta$ in central $\mathrm{Au}-\mathrm{Au}$ collisions at $\sqrt{s_{\mathrm{NN}}}=200 \mathrm{GeV}$ at RHIC the lower limit of the energy density can be estimated to be around $\epsilon_{B}=5.4 \mathrm{GeV} \mathrm{fm}^{-3}$ assuming $\tau=1 \mathrm{fm} / c$ [44]. This exceeds the critical value by a factor of five and thus the conditions for the formation of a QGP is fulfilled. With the much higher center-of-mass energy at the LHC, a density of $\epsilon_{B}=14 \mathrm{GeV} \mathrm{fm}^{-3}$ can be reached in $\mathrm{Pb}-\mathrm{Pb}$ collisions at $\sqrt{s_{\mathrm{NN}}}=2.76 \mathrm{TeV}$ exceeding the critical density by an order of magnitude [48].

\subsubsection{Collective flow}

The overlap region of two colliding nuclei can be of nonspherical symmetry, which is especially the case for noncentral collisions. Due to this, anisotropic pressure gradients are introduced in the created medium which result in an anisotropic expansion which is stronger in the reaction plane direction and referred to as collective flow [50-52]. The reaction plane is defined as the plane spanned by a line connecting the centers of both nuclei and the beam axis. The distance between the centers of the nuclei is thereby defined as the impact parameter $b$. A schematic view of the evolution of the medium due to the pressure gradients is shown in Figure 2.8 where the almond-shaped overlap region $(\mathrm{red})$ is shown as well as the spectator parts of the nuclei (blue). Fourier expansions [53] can be used to describe the azimuthal asymmetry via

$$
\frac{\mathrm{d} N}{\mathrm{~d} \varphi}=1+2 \sum_{n=1}^{\infty} \nu_{n} \cos \left[n\left(\varphi-\Psi_{n}\right)\right],
$$

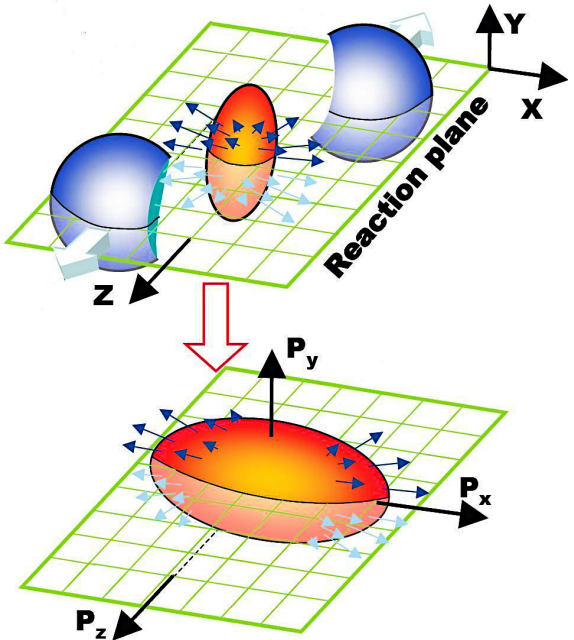

Figure 2.8: Evolution of the almond shaped interaction volume from a semi-central collision of two nuclei relative to the reaction plane due to the internal pressure gradients. Taken from [49].

where the azimuthal angle of the particle is given by $\varphi$, the azimuthal orientation of the reaction plane by $\Psi_{n}$ and the order of the harmonic by $n$. The ideal almond shape of the overlap region is dissolved into different shapes due to the flow as well as the inhomogeneous distribution of partons in the colliding nuclei. This effect is present on an event-by-event basis where pressure gradients as well as participants in the collision vary. One can therefore express higher order Fourier coefficients $\nu_{n}$ for the azimuthal anisotropy of these different shapes via

$$
\nu_{n}=\left\langle\cos \left[n\left(\varphi-\mid \Psi_{n}\right)\right]\right\rangle
$$

The first three harmonics $(n=1,2,3)$ are referred to as the direct, elliptic and triangular flow, respectively. The dominant contribution comes from $\nu_{2}$ as it is directly linked to the almond 

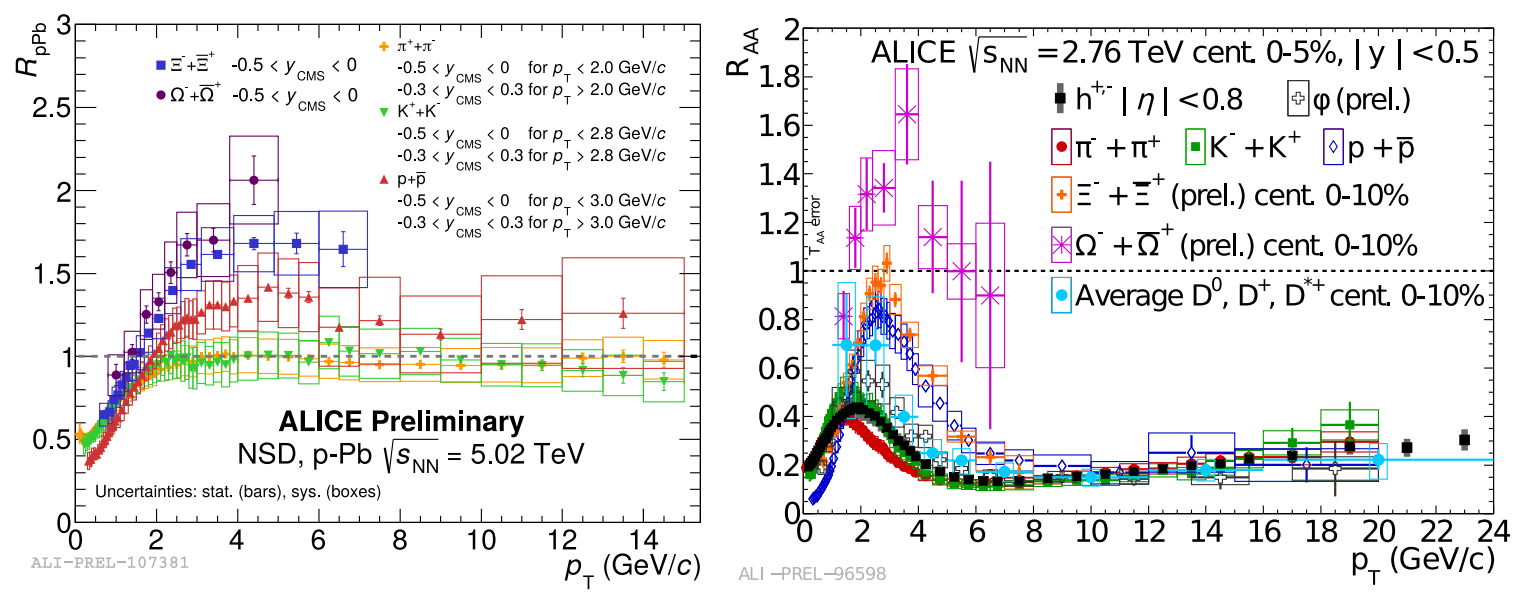

Figure 2.9: Left: Nuclear modification factor $R_{\mathrm{pA}}$ for identified charged hadrons [57] as well as for $\Omega$ and $\Xi$ [58] baryons in $\mathrm{p}-\mathrm{Pb}$ collisions at $\sqrt{s_{\mathrm{NN}}}=5.02 \mathrm{TeV}$. Right: Nuclear modification factor $R_{\mathrm{AA}}$ for multiple identified particle species in central $\mathrm{Pb}-\mathrm{Pb}$ collisions at $\sqrt{s_{\mathrm{NN}}}=2.76$ $\mathrm{TeV}$ [59-62].

shape of the medium while $\nu_{1}$ is concentrated towards beam rapidity [54]. Measurements of the elliptic flow and its centrality dependence can provide constraints on the equation of state, on the initial conditions of the hydrodynamic evolution as well as on the value of $\eta / s[55,56]$.

\subsubsection{Modification of particle production}

The key result of this thesis as well as one of the most indicative observables for the QGP is the nuclear modification factor $R_{\mathrm{pA}}$ or $R_{\mathrm{AA}}$ for $\mathrm{p}-\mathrm{A}$ and $\mathrm{A}-\mathrm{A}$ collisions, respectively. It quantifies how the particle production in a heavy-ion collision deviates from a simple superposition of individual nucleon collisions if the values of $R_{\mathrm{pA}}$ or $R_{\mathrm{AA}}$ are not equal to unity. The nuclear modification factor itself is defined as the ratio of the measured yield in the larger collision system $(X=\mathrm{pA}$ or AA) to the binary collision scaled reference yield from pp collisions at the same center-of-mass energy. It is defined as

$$
R_{\mathrm{X}}\left(p_{\mathrm{T}}\right)=\frac{\mathrm{d}^{2} N^{\mathrm{X}} / \mathrm{d} p_{\mathrm{T}} \mathrm{d} y}{\left\langle T_{\mathrm{X}}\right\rangle \mathrm{d}^{2} \sigma^{\mathrm{pp}} / \mathrm{d} p_{\mathrm{T}} \mathrm{d} y},
$$

where $\mathrm{d}^{2} N^{\mathrm{X}} / \mathrm{d} p_{\mathrm{T}} \mathrm{d} y$ is the double differential invariant yield of a given particle species in the larger collision system while $\sigma^{\mathrm{pp}} / \mathrm{d} p_{\mathrm{T}} \mathrm{d} y$ represents the invariant cross section for the same particle measured in the reference pp system. The average nuclear overlap function (or nuclear thickness) $\left\langle T_{\mathrm{X}}\right\rangle$ is related to the mean number of collisions via $\left\langle T_{\mathrm{X}}\right\rangle=\frac{\left\langle N_{\text {coll }}\right\rangle}{\sigma^{\mathrm{Pp}}}$ with $\left\langle N_{\text {coll }}\right\rangle$ being calculated using Glauber Monte-Carlo model simulations [37] and $\sigma^{\text {pp }}$ being the production cross section in the reference pp system.

A selection of nuclear modification factors for various particle species is shown in Figure 2.9 for $\mathrm{p}-\mathrm{Pb}$ collisions at $\sqrt{s_{\mathrm{NN}}}=5.02 \mathrm{TeV}$ (left) $[57,58]$ and central $\mathrm{Pb}-\mathrm{Pb}$ collisions at $\sqrt{s_{\mathrm{NN}}}=2.76$ $\mathrm{TeV}$ (right) [59-62]. As can be seen, the $R_{\mathrm{pA}}$ and $R_{\mathrm{AA}}$ show distinct deviations from unity where a suppression at low $p_{\mathrm{T}}$ is observed in all systems while for all particle species at intermediate $p_{\mathrm{T}}$ a particle species dependent behavior of suppression or enhancement is seen. At high $p_{\mathrm{T}}$, the nuclear modification factors in $\mathrm{p}-\mathrm{Pb}$ collisions are found to be consistent with unity while a strong suppression is seen in the heavy-ion collision measurements. The modifications of the particle yield are not only influenced by final state parton energy loss but also initial-state effects. In the following, the main initial and final state effects are introduced and their relevance for small and large systems is given. 


\subsubsection{Cronin effect and multiple scattering}

The enhancement seen in Figure 2.9 in the intermediate $p_{\mathrm{T}}$ region of $1-3 \mathrm{GeV} / c$ is largely attributed to the Cronin effect [63]. The hardening of the spectrum in proton-nucleus collisions was first observed in the 1970s which was accompanied by a depletion of particle yield at lower transverse momentum. This effect is interpreted as the increase of the protons parton transverse momentum $k_{\mathrm{T}}$ via multiple scatterings off partons from the nucleus [64] which leads to an increase of the transverse momentum of the afterwards produced hadron. For high $k_{\mathrm{T}}$, multiple scattering effects are suppressed as higher twist effects [65], which are described in pQCD and are antiproportional to powers of $k_{\mathrm{T}}$, become relevant and the additional transverse momentum due to the scatterings becomes negligible relative to the initial $p_{\mathrm{T}}$. The Cronin effect furthermore shows a dependence on the center-of-mass energy of the initial collision which in turn allows for a larger momentum transfer $Q^{2}$ as well as a dependence on the rapidity region which influences the $x$ values that are probed as shown in measurements by the BRAHMS collaboration $[66,67]$. These measurements showed that for large rapidities the Cronin peak is less pronounced leading even to a suppression at very high rapidities. Around mid-rapidity the Cronin effect shows a centrality dependence with a larger peak at more central collisions while this effect is inverted when going to forward rapidities [68]. As shown in Figure 2.9, the Cronin effect is stronger for baryons than for mesons which points to an additional dependence on the baryon production mechanism.

\subsubsection{Nuclear parton distribution functions}

The cross section for the production of a given hadron depends on the parton distribution functions (PDF) of the partons that take part in the collision of the nuclei. The PDFs $f_{i}^{p}\left(x, Q^{2}\right)$ describe the density of the different partons ( $i=$ quark or gluon) within the hadron at a given momentum fraction $x$ of the hadron momentum and the hard interaction momentum transfer $Q^{2}$ [69]. As shown in Figure 2.10, at low $x$ the PDFs, especially for the gluon distribution, show a strong dependence on $Q^{2}$ which can be interpreted as an increase in resolution of the proton which reveals more low momentum partons. Above a certain $Q^{2}$ and at low $x$, saturation effects on the gluon density are expected to appear where the gluons begin to interact with themselves and can fuse $(g g \rightarrow g)$. This effect can be described by the Color Glass Condensate (CGC) [70] field theory which treats valence quarks as static color sources and the dynamically produced gluons at momenta below a saturation scale $Q_{\text {sat }}$ with classical field theory. The presence of this effect is yet to be further experimentally constrained due to a lack of measurements to impose limits in the current predictions. PDFs can not be calculated from first principles, but can be experimentally determined via Deep Inelastic Scattering (DIS) [69, 72,73] of leptons off protons. The thereby measured structure functions allow to obtain parametrized PDFs which can be evolved using the DGLAP equation [74-76] in $Q^{2}$. For partons bound in a nuleon, the PDFs can change which in turn impacts directly the particle yield. The nuclear PDFs

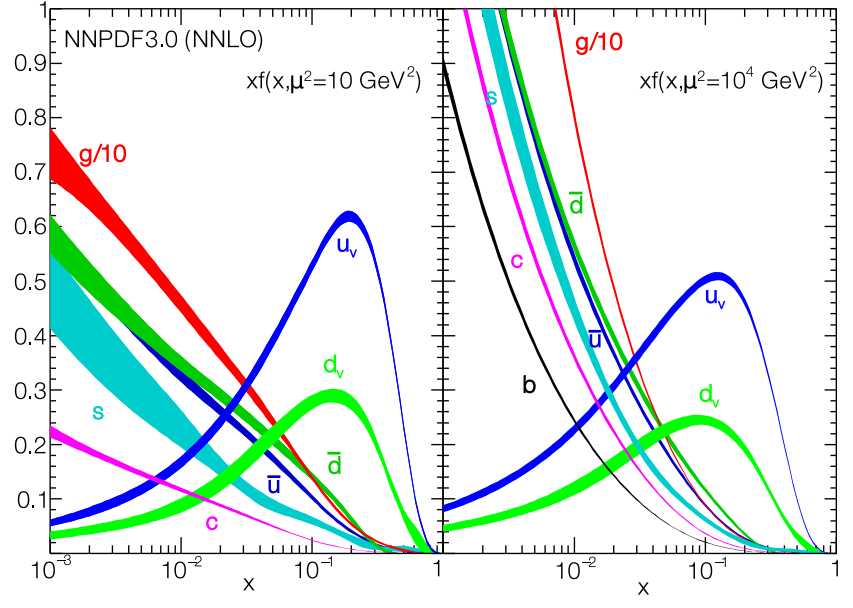

Figure 2.10: Parton distribution functions $x \cdot f(x)$ from the NNLO NNPDF3.0 global analysis [71] for $Q^{2}=10 \mathrm{GeV}^{2}$ (left) and $Q^{2}=10^{4} \mathrm{GeV}^{2}$ (right). 
(nPDF) can be expressed as

$$
f_{i}^{A}\left(x, Q^{2}\right)=R_{i}\left(A, x, Q^{2}\right) f_{i}^{p}\left(x, Q^{2}\right),
$$

where $R_{i}$ denotes the modification relative to the proton $\mathrm{PDF} f_{i}^{p}$. This initial state nuclear modification has been measured by comparisons of the structure functions obtained from DIS of deuterons up to heavy nuclei like iron which resulted in a maximum depletion of $\sim 20 \%$ for $0.5<x<0.8$ $[78,79]$. Figure 2.10 shows proton nuclear parton distribution functions at $Q^{2}=10$ $\mathrm{GeV}^{2}$ (left) and at $Q^{2}=10^{4} \mathrm{GeV}^{2}$ (right) highlighting the $Q^{2}$ and $x$ dependence of the various quark and gluon PDFs. Furthermore, in the nuclear PDFs for different collision energies the parton densities can

be depleted or enhanced depending on the $x$ and $Q^{2}$ values which are predominantly probed at the given center-of-mass energy. This effect is called shadowing (for the depletion) for $x<0.1$ and anti-shadowing (for the enhancement) around $x \approx 0.15$ [80]. Figure 2.11 shows this effect for the nCTEQ15 [77] nPDF at $Q=10 \mathrm{GeV}$ in terms of a nuclear modification factor defined as the ratio of the proton PDFs bound in a lead ion to the free proton PDFs. The ratio exhibits values below unity for low $x$ in the shadowing region while an enhancement in the anti-shadowing region $(x \sim 0.1)$ is visible. As a consequence, a depletion of soft particle yield and enhancement of mid $p_{\mathrm{T}}$ yield is expected in collisions involving a lead ion compared to purely proton-proton collisions. The dependence of $x$ on the center-of-mass energy of the collision is given by $x=\frac{m_{\mathrm{T}}}{\sqrt{s}} \exp ( \pm y)$ and therefore the shadowing effect at RHIC energies is only present for $p_{\mathrm{T}}<1-2 \mathrm{GeV} / c$ while at LHC energies the effect covers a larger momentum region. Measurements of the nuclear modification factor $R_{X}\left(p_{\mathrm{T}}\right)$ as given in Equation 5 for different particle species at various center-of-mass energies as well as over large transverse momentum ranges can therefore help constrain nuclear effects in the PDFs.

\subsubsection{Energy loss in cold and hot nuclear matter}

From the nuclear modification factor measurements presented in Figure 2.9 a strong suppression of single particle yield is observed in heavy-ion collisions compared to similar measurements in p-A collisions. This particle suppression, first fully observed at RHIC [81,82], is attributed to jet quenching and therefore strengthens the assumption that a dense medium is created in such collisions which leads to parton energy loss via medium interactions. These interactions can be categorized into either energy loss via collisions in the medium or as radiative energy loss. Collisional energy loss is the dominant process at low energies and describes elastic collisions of the hard parton with the partons of the medium. These elastic scatterings can be described by

$$
\langle\Delta E\rangle_{\mathrm{scatt}}=N \cdot\langle\Delta E\rangle \propto L,
$$

where $N=\sigma \rho L$ is the number of elastic scatters, calculated from the scatter density $\rho$, the elastic scattering cross section $\sigma$ and the path length in the medium $L$. As elastic scatterings are not coherent, only the average energy loss for one scattering process, given by $\langle E\rangle$, can be determined. Furthermore, the cross section in Equation 7 depends on color charge and is therefore smaller for quarks than for gluons. This is explained in Refs. [83, 84] via the Casimir factor $C_{r}$ which is $4 / 3$ for a quark-gluon interaction while it is 3 for a gluon-gluon interaction. 
The collisional energy loss is, however, not the dominant mechanism for light quarks and gluons or generally at high parton momentum. Instead the in-medium energy loss is dominated by the radiation of gluons via gluon bremsstrahlung which can be described as

$$
\langle\Delta E\rangle_{\text {radiative }} \propto m_{D}^{2} \rho \sigma L^{2} \propto L^{2},
$$

where $m_{D}^{2} \rho \sigma=\hat{q}$ is the transport coefficient that describes the aquired momentum in the medium which in turn can be emitted as a gluon. The dependence on $L^{2}$ shows the larger magnitude of gluon radiation compared to collisional energy loss. Overall, gluons experience the strongest radiative energy loss in the medium followed by the quarks in increasing mass order.

\subsubsection{Electromagnetic probes}

Electromagnetic probes such as photons are ideal to access the evolution of a high energetic or heavy-ion collision as they are produced during all stages and are not impacted significantly by strong final state interactions as they only interact electromagnetically [85]. Combined with their mean free path length that is much larger than the medium itself this allows them to carry information about the strongly interacting medium outwards [86]. In an experimental environment it is only possible to measure the inclusive photon sample which is the sum of several components originating from different production mechanisms. The inclusive photons can be separated into the decay photons $\left(\gamma_{\mathrm{dec}}\right)$, which are composed of all photons from hadron decays, and the direct photons $\left(\gamma_{\text {dir }}\right)$ which are all photons that do not originate from particle decays. The direct photons are further subdivided into fragmentation, prompt and thermal photon components as shown in Figure 2.12. For $p_{\mathrm{T}}>3 \mathrm{GeV} / c$, photons are dominantly produced in hard $2 \rightarrow 2$ scattering processes like Compton scattering or quark-antiquark annihilation which can be calculated within pQCD. Another feature of these $2 \rightarrow 2$ processes (e.g. $q g \rightarrow q \gamma$ or $q \bar{q} \rightarrow g \gamma$ ) is that the produced quark or gluon component of the scattering process will produce with a high probability a jet during hadronization. As the quark or gluon is subject to the strong interaction, its energy can be strongly reduced by interactions in the medium, whereas the outgoing photon remains unaffected. Measurements of $\gamma$-jet correlations therefore allow to probe the parton distribution functions and to get a handle on the energy loss effects in the medium as the unmodified photon provides a reference for the opposing parton energy. In systems where a sizeable QGP is produced, like A-A collisions or possibly high multiplicity $\mathrm{p}-\mathrm{A}$ collisions, further photon production mechanisms are contributing in terms of jet-medium interactions and thermal photon emission. Photons from jet-medium interactions are produced when hard partons (the jet) interact with the thermalized partons in the hot medium via Compton scattering, annihilation or bremsstrahlung. Thermal photons are emitted from the QGP and the hadron gas similar to black body radiation and are the dominant contribution at very low momentum following an exponential spectral shape as seen in Figure 2.12. Their spectrum is dependent on the temperature of the medium but as the medium is rapidly expanding and cooling down

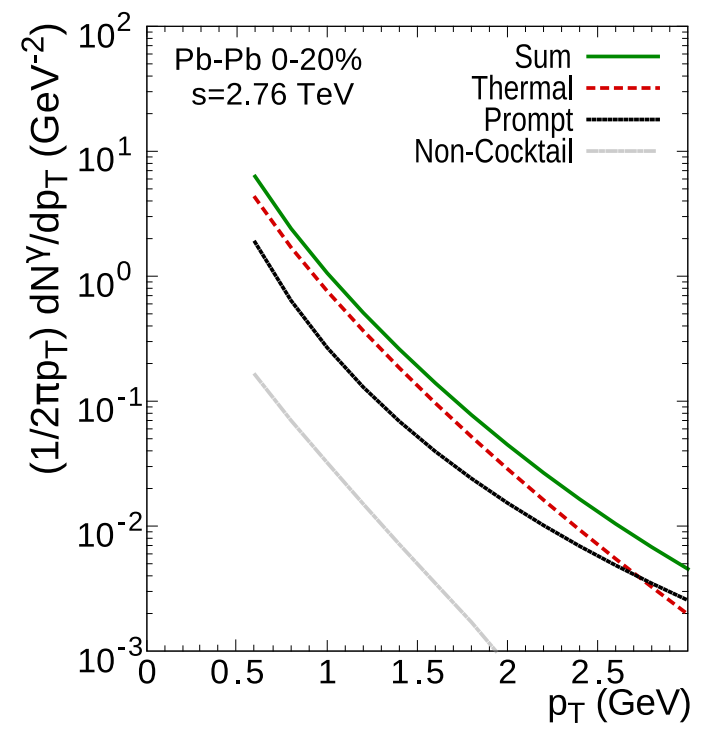

Figure 2.12: Invariant yield at midrapidity of the different direct photon components in $\mathrm{Pb}-\mathrm{Pb}$ collisions at an LHC energy of $\sqrt{s_{\mathrm{NN}}}=2.76 \mathrm{TeV}[87,88]$. 

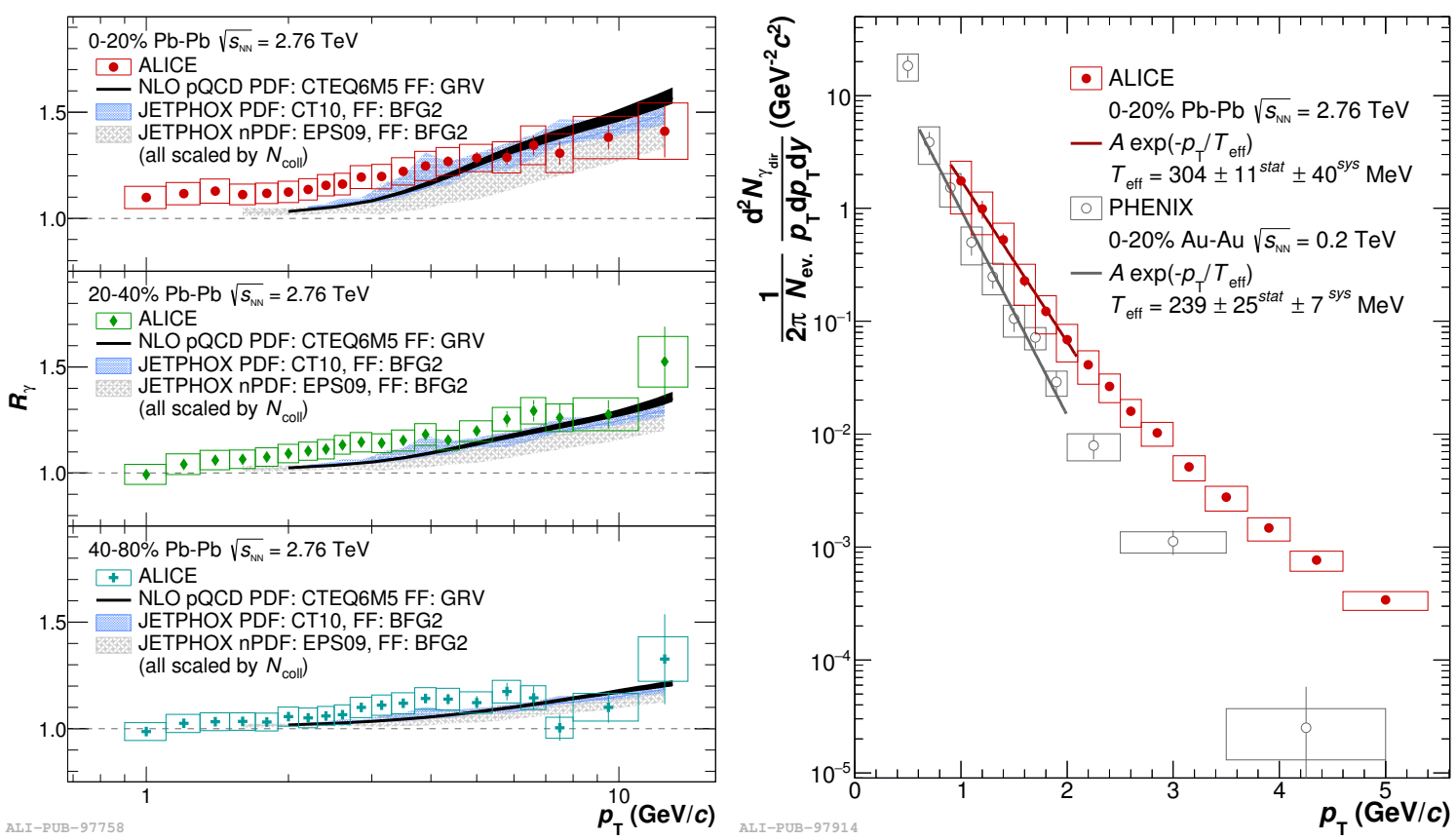

Figure 2.13: Left: Direct photon excess ratio $R_{\gamma}$ measured in three different centrality classes in $\mathrm{Pb}-\mathrm{Pb}$ collisions at $\sqrt{s_{\mathrm{NN}}}=2.76 \mathrm{TeV}$ together with a variety of theory predictions [89]. Right: Direct photon differential invariant yield in $0-20 \%$ central $\mathrm{Pb}-\mathrm{Pb}$ collisions at $\sqrt{s_{\mathrm{NN}}}=2.76$ $\mathrm{TeV}$ [89] and $\mathrm{Au}-\mathrm{Au}$ collisions at $\sqrt{s_{\mathrm{NN}}}=200 \mathrm{GeV}$ [90] together with an exponential fit on the thermal photon signal.

after its formation, only an effective temperature can be determined from the thermal photon spectral shape.

In order to access the direct photon signals experimentally it is necessary to measure the inclusive photon sample and subtract the decay photons from this sample. Therefore a subtraction method [91] is used according to

$$
\gamma_{\mathrm{dir}}=\gamma_{\mathrm{inc}}-\gamma_{\mathrm{dec}}=\left(1-R_{\gamma}^{-1}\right) \cdot \gamma_{\mathrm{inc}},
$$

where the ratio of inclusive over decay photons is defined as $R_{\gamma} \equiv \gamma_{\text {inc }} / \gamma_{\text {dec }}$ and called the direct photon excess ratio. The decay photon spectrum $\gamma_{\mathrm{dec}}$ is obtained from a particle decay simulation using parametrizations of measured particle spectra or $m_{\mathrm{T}}$ scaled [92] spectra as inputs in order to correctly describe the contributions of each particle. This so-called decay photon cocktail is dominated by contributions from the light neutral mesons $\pi^{0}(\sim 80-85 \%), \eta(\sim 10-15 \%)$ and $\omega(\sim 3 \%)$. The direct photon excess ratio is moreover approximated and extended into a double ratio defined as

$$
R_{\gamma} \approx \frac{\left(\gamma_{\text {inc }} / \pi^{0}\right)_{\text {meas }}}{\left(\gamma_{\text {dec }} / \pi_{\text {param }}^{0}\right)_{\text {cocktail }}}
$$

where the addition of the $\pi^{0}$ spectrum provides an internal normalization and allows to catch possible biases that would affect both measurements. Furthermore, this double ratio allows for strong cancellations of systematic uncertainties that are shared between the $\gamma_{\text {inc }}$ and the $\pi^{0}$ meson measurement $[92,93]$.

Direct photon measurements from ALICE in three centrality classes [89] can be seen in Figure 2.13. The direct photon excess ratio is shown in the left plot while the direct photon spectrum in $0-20 \%$ centrality $\mathrm{Pb}-\mathrm{Pb}$ collisions at $\sqrt{s_{\mathrm{NN}}}=2.76 \mathrm{TeV}$ is shown on the right side compared to a similar measurement from PHENIX [90] in 0-20\% Au-Au collisions at $\sqrt{s_{\mathrm{NN}}}=200 \mathrm{GeV}$. 
A clear excess of direct photons in all centrality classes at high $p_{\mathrm{T}}$ is seen, given by $R_{\gamma}$ values greater than unity. The measured excess is well described by various theoretical predictions. In central and semicentral collisions an excess of direct photons is seen at low $p_{\mathrm{T}}$, which is attributed to thermal radiation of the QGP. The excess is about $5-8 \%$ in semi-central collisions and reaches up to $13 \%$ in central collisions which, given the total uncertainties of the measurements, results in a thermal photon signal with $2.6 \sigma$ significance in the latter case. The differential invariant yield of direct photons at low momentum, shown in Figure 2.13 (right), can be described by an exponential $\propto \exp \left(-p_{\mathrm{T}} / T\right)$ which is fitted in the $p_{\mathrm{T}}$ range of $0.9<p_{\mathrm{T}}<2.1 \mathrm{GeV} / c$. From the fit, the effective temperature $T_{\text {eff }}=304 \pm 11^{\text {stat }} \pm 40^{\text {sys }} \mathrm{MeV}$ can be obtained from the inverse slope parameter $T$. This temperature is well above the critical temperature, as described in Section 2.2, and higher than the temperature of $T_{\text {eff }}=239 \pm 25^{\text {stat }} \pm 7^{\text {sys }} \mathrm{MeV}$ measured in $\mathrm{Au}-\mathrm{Au}$ collisions at $\sqrt{s_{\mathrm{NN}}}=200 \mathrm{GeV}$ by the PHENIX collaboration.

Aside from the direct photon spectra which provide access to the initial temperature of the QGP, also measurements of the direction photon flow are performed. The measurements follow the same basic principles as described in Section 2.3.2 and Equation 4 to access the flow $\nu$ of inclusive, decay and direct photons. Similar to the direct photon spectra, the direct photon flow is accessed via comparisons of the inclusive and the decay photon flow according to

$$
\nu_{2}^{\gamma_{\mathrm{dir}}}=\frac{R_{\gamma} \cdot \nu_{2}^{\gamma_{\mathrm{inc}}}-\nu_{2}^{\gamma_{\mathrm{dec}}}}{R_{\gamma}-1}
$$

It was expected that direct photons do not experience a substantial flow but first measurements by
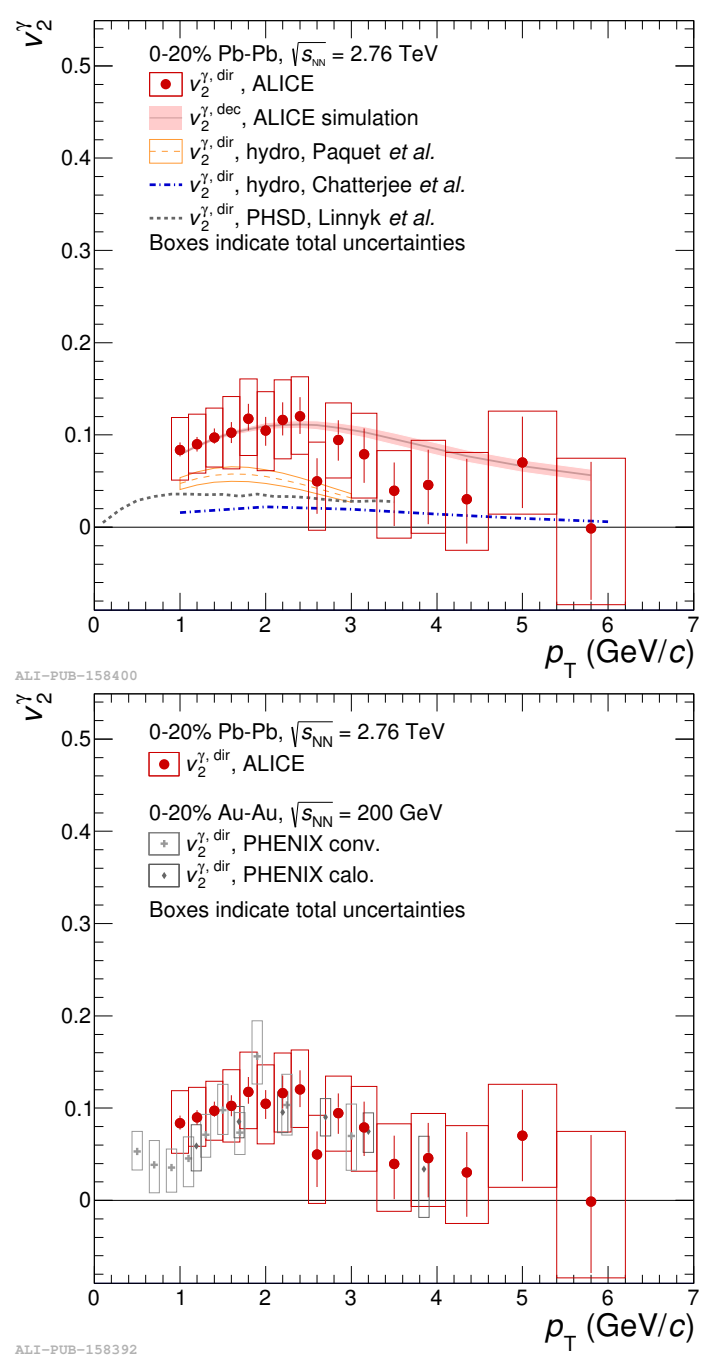

Figure 2.14: Top: Direct, inclusive and decay photon $\nu_{2}$ in central $\mathrm{Pb}-\mathrm{Pb}$ collisions at $\sqrt{s_{\mathrm{NN}}}=2.76 \mathrm{TeV}$ together with theory predictions. Bottom: Direct photon flow $\nu_{2}$ in central $\mathrm{Pb}-\mathrm{Pb}$ and $\mathrm{Au}-\mathrm{Au}$ collisions by ALICE and PHENIX at low $p_{\mathrm{T}}[94-96]$.

the PHENIX collaboration $[94,95]$ determined the

direct photon $\nu_{2}$ and $\nu_{3}$ to be of a similar magnitude as for charged particles. The measurements from PHENIX and ALICE [96] together with various theoretical model predictions are shown in Figure 2.14 The large flow measurements point to a late production of the direct photons when the flow is already established [97]. This stands in tension with the slope and absolute amount of measured direct photons that originate from an early production off the hot QGP. This resulted in the so-called direct photon puzzle $[98,99]$ which is neither supported nor opposed by ALICE measurements in central $\mathrm{Pb}-\mathrm{Pb}$ collisions [96] where the effect was only observed with $1.4 \sigma$. Higher precision measurements in the large data dample of $\mathrm{Pb}-\mathrm{Pb}$ collisions at $\sqrt{s_{\mathrm{NN}}}=5.02 \mathrm{TeV}$ recorded in 2018 should provide a significant constrain to this effect.

In addition to the measurements in heavy-ion collisions it remains to be seen if a thermal photon excess and possible flow can be observed in small systems like $\mathrm{p}-\mathrm{Pb}$. Recent measurements by PHENIX in minimum bias $\mathrm{d}-\mathrm{Au}$ collisions at $\sqrt{s_{\mathrm{NN}}}=200 \mathrm{GeV}$ [102] determined a direct 

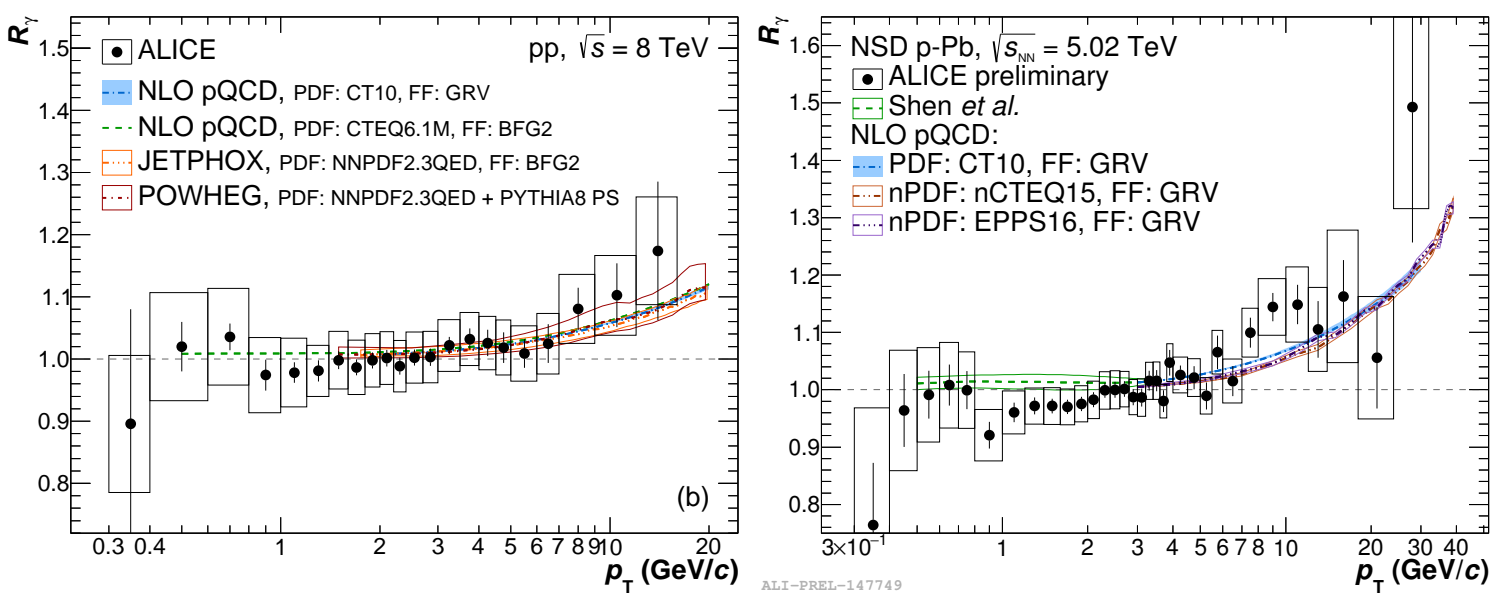

Figure 2.15: Direct photon excess ratio in pp collisions at $\sqrt{s}=8 \mathrm{TeV}$ [100] (left) and in minimum bias p-Pb collisions at $\sqrt{s_{\mathrm{NN}}}=5.02 \mathrm{TeV}[88,101]$ (right) measured by ALICE together with various theory predictions.

photon signal consistent with theory predictions but also still limited by statistical and systematic precision. Similar measurements performed by ALICE in pp collisions at $\sqrt{s}=2.76$ and $8 \mathrm{TeV}$ as well as in minimum bias $\mathrm{p}-\mathrm{Pb}$ collisions at $\sqrt{s_{\mathrm{NN}}}=5.02 \mathrm{TeV}$ did not show significant direct photon signals at low $p_{\mathrm{T}}$ as shown in Figure 2.15. The multiplicity dependent direct photon measurements in $\mathrm{p}-\mathrm{Pb}$ collisions at $\sqrt{s_{\mathrm{NN}}}=5.02 \mathrm{TeV}$ presented in Chapter 7 therefore aim to put constrains on the thermal photon production in small collision systems at LHC energies. 


\section{Experimental Overview}

CERN was founded in 1954 close to Geneva, Switzerland and has by now expanded to 22 member states and is composed of 3430 on-site scientists, engineers and further staff with additional 17663 external users which have access to computing and experimental resources. Furthermore, CERN is open to the public within guided tours of experiments and exhibitions bringing about 110,000 visitors every year in contact with its science program.

This chapter focuses on the description of the experimental setup at CERN and its various components ranging from the particle accelerator chain to the subsystems of the ALICE detector. In addition, key features of the ALICE detector for data taking and reconstruction will be explained.

\subsection{The Large Hadron Collider}

The worlds largest and highest energetic particle accelerator is the LHC at CERN, located at the French-Swiss border near Geneva [104] as shown in Figure 3.1. With the goal to explore physics beyond the Standard Model, the accelerator was designed to reach center-of-mass energies of up to $\sqrt{s}=14 \mathrm{TeV}$ for protons and up to $\sqrt{s_{\mathrm{NN}}}=5.5 \mathrm{TeV}$ for heavy ions including lead, argon, xenon and more. In order to supply the high luminosity experiments ATLAS [105] and CMS [106], a peak design luminosity of $\mathscr{L}=$ $10^{34} \mathrm{~cm}^{-2} \mathrm{~s}^{-1}$ was opted for in the case of proton-proton collisions and $\mathscr{L}=10^{27} \mathrm{~cm}^{-2} \mathrm{~s}^{-1}$ for $\mathrm{Pb}$-ion collisions. The luminosity is calculated as

$\mathscr{L}=\frac{N^{2} k f \gamma}{4 \pi \epsilon_{n} \beta} \cdot\left[1+\left(\frac{\theta \sigma^{\prime}}{2 \sigma *}\right)^{2}\right]^{-1 / 2}$

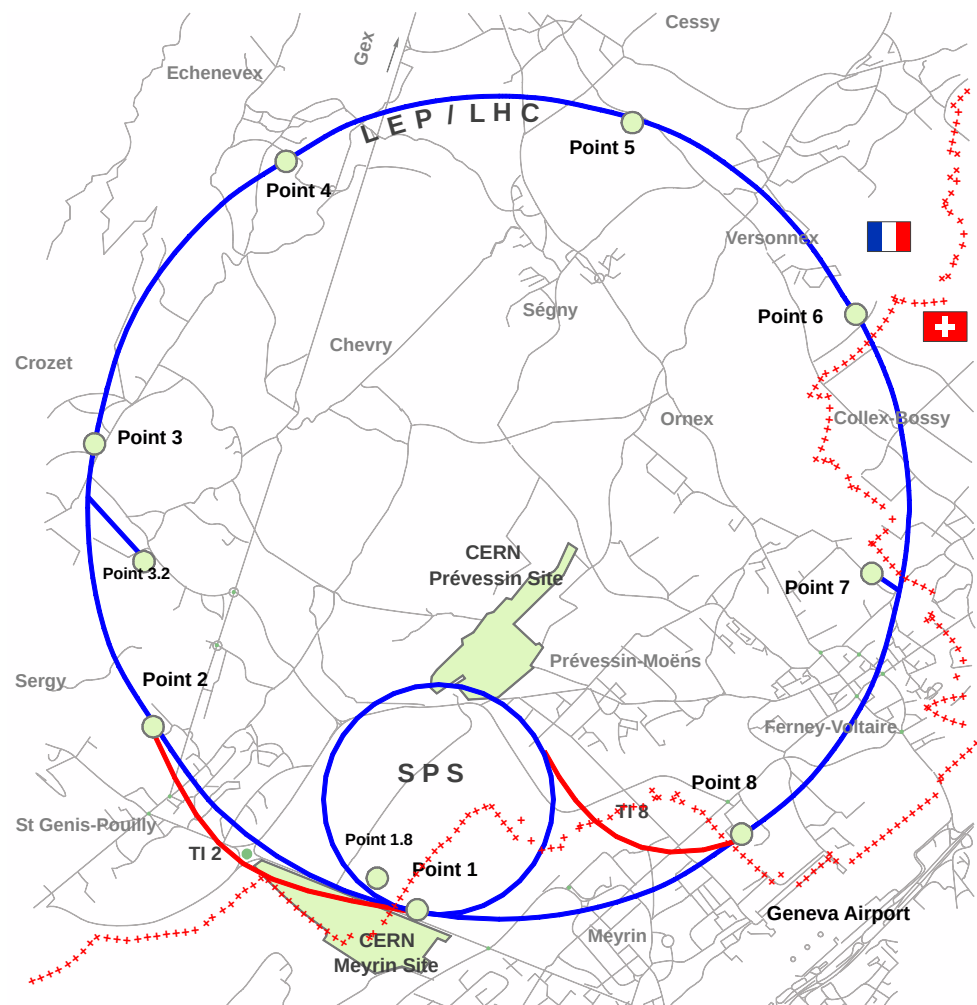

Figure 3.1: Map of the French and Swiss area with the LHC and its access points. Derived from [103].

where $N_{\text {part }}$ stands for the amount of particles per bunch, $k$ for the number of bunches per beam, $f$ for the frequency with which the beam revolves in the LHC, $\gamma$ as the relativistic gamma factor, and $\epsilon_{n} \beta$ for the product of beam emittance and beta function at the collision point. The term in the square brackets represents the geometric luminosity reduction factor which has to be added as the beams are brought to collision at a certain crossing angle $\theta$ with RMS bunch length $\sigma^{\prime}$ and transverse RMS beam size $\sigma *$. Center-of-mass energy as well as luminosity of the LHC were optimized in order to provide the necessary conditions and data to observe and furthermore probe the Higgs boson which was expected to have a mass between 115 and $145 \mathrm{GeV}$. The collider spans a circumference of about $27 \mathrm{~km}$ and is located at varying depth due to the proximity to the Jura mountains from 45 to $170 \mathrm{~m}$ underground. The tunnel for the 
LHC was re-purposed from the previous accelerator LEP, which operated until the year 2000, thus resulting in a significantly lowered overall build cost but imposing the restriction that there can not be two fully separate acceleration rings for the beams due to the tunnel dimensions. The tunnel consists of eight bent and eight straight sections and therefore forms a decagon instead of an actual ring. Dipole bending magnets cooled with liquid helium to $1.9 \mathrm{~K}$ and operated at up to $8.3 \mathrm{~T}$ field strength keep the beams in their orbit. They are located in the eight bent parts of the accelerator ring. Further multipole magnets are used to focus and stabilize the beam in the straight LHC sections to ensure a proper orbit.

An ultra-high vacuum of $10^{-10} \mathrm{mbar}$ is created in the beam pipes in order to keep nuclear scatterings with residual gases to an absolute minimum. With this vacuum, only a very small nuclear scattering probability in the residual $\mathrm{H}_{2}$, helium, $\mathrm{CO}_{2}$ and other gases is present resulting in $<30 \mathrm{~mW} \mathrm{~m}^{-1}$ energy loss per beam which is an order of magnitude less than the combined 0.4 $\mathrm{W} \mathrm{m}{ }^{-1}$ per beam from synchrotron light radiation and image currents [104].

The first stable beams in the LHC were achieved on 10 September 2008 but were unfortunately followed 9 days later by the LHC magnet quench incident [107] which destroyed 53 magnets as a consequence. This incident delayed the LHC time plan by more than a year resulting in the first proton-proton collisions at $\sqrt{s}=0.9 \mathrm{TeV}$ in November of the following year. During the LHC Run 1 (2008-2013), a maximum collision energy of $\sqrt{s}=8 \mathrm{TeV}$ was reached for pp collisions. Repairs and improvements on the accelerator during the first long shutdown should ensure a safe operation at the LHC design energies during Run 2. Furthermore, in November 2010 the first $\mathrm{Pb}$-ion beams were brought to collisions at a center-of-mass energy of $\sqrt{s_{\mathrm{NN}}}=2.76 \mathrm{TeV}$.

In the following sections, the full chain for LHC beam preparation as well as beam injection is briefly explained followed by an overview of the LHC interaction points.

\subsubsection{Proton and ion source}

The protons circulating in the LHC originate from a bottle of hydrogen located at the linear accelerator Linac $2[109,110]$ which also serves as the first accelerator in the injection scheme of the LHC as described in the next section [108]. In the duoplasmatron source [111], installed in 1992, the hydrogen atoms are stripped from their electron via an electrical discharge between a heated cathode at $91 \mathrm{kV}$ and an anode at $-3 \mathrm{kV}$ within a magnetic field and subsequently accelerated to $50 \mathrm{MeV}$ for injection into the Proton Synchrotron Booster (PSB). The source is shown schematically in Figure 3.2 with its various components

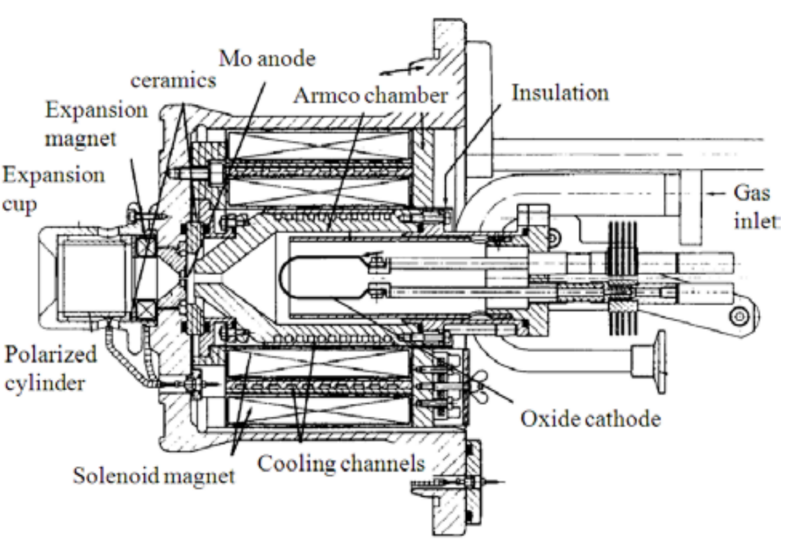

Figure 3.2: Slice view of the duoplasmatron proton source at the Linac 2 at CERN [108].

labeled. The heavier lead ions are obtained from an Electron Cyclotron Resonance Ion Source (ECRIS) [112] and are accelerated with the Linac 3 [113] to be injected into the Low Energy Ion Ring (LEIR) [114] for cooling and accumulation. For the ion production, a solid lead strip of 2 grams is slowly evaporated in micro ovens and the resulting Pb-gas is mixed with oxygen as a support gas. The electrons are stripped similarly to the hydrogen atoms for the proton beam in an electric field of varying strength depending on the ion ( $\mathrm{Pb}, \mathrm{Xe}, \mathrm{O}$ or $\mathrm{In})$. As high luminosities are desired in the LHC experiments, the heavy ions are accumulated in LEIR to be able to inject the maximum of 592 bunches needed into the LHC in a short time frame. Furthermore, LEIR provides electron cooling in order to reduce the emittance of the ion beam. 


\subsubsection{Injection scheme}

The electron stripped protons and heavy ions undergo several acceleration steps before they are injected into the LHC for collisions. A full schematic overview of the injection scheme is shown in Figure 3.3. The first acceleration steps are different for protons and heavy ions; For protons, the $80 \mathrm{~m}$ long linear accelerator Linac 2 accelerates them first to $50 \mathrm{MeV}$ from which they are inserted into the Proton Synchrotron Booster (PSB) [115] where they are brought up to 1.4 $\mathrm{GeV}$ before being injected into the Proton Synchrotron (PS) [116]. The PSB is made of four superimposed cyclotron rings of $25 \mathrm{~m}$ radius where the protons gain about $1 \mathrm{keV}$ of energy in each turn. Furthermore, the usage of the PSB allows over 100 times more protons to be accepted by the PS. For heavy ions, the initial acceleration from the Linac 3 is $4.2 \mathrm{MeV}$ per nucleon with which they are stored in LEIR which further accelerates them to $72.2 \mathrm{MeV}$ per nucleon.

The Proton Synchrotron accelerates protons up to $25 \mathrm{GeV}$ and $\mathrm{Pb}$-ions to $5.9 \mathrm{GeV}$ per nucleon while also stripping further electrons from the heavy ions. Its name originates from the fact that it is a cyclotron with synchronized increase of the beam bending with increasing beam energy. With its circumference of $628 \mathrm{~m}$ and the beam energies it can achieve, it was the worlds strongest particle accelerator when it became operational in 1959. During the 1990s the PS was modified to supply leptons for LEP with energies up to $3.5 \mathrm{GeV}$ followed by a full remodeling for the LHC era where all magnets were replaced.

From the PS, the protons or heavy ions are injected into the Super

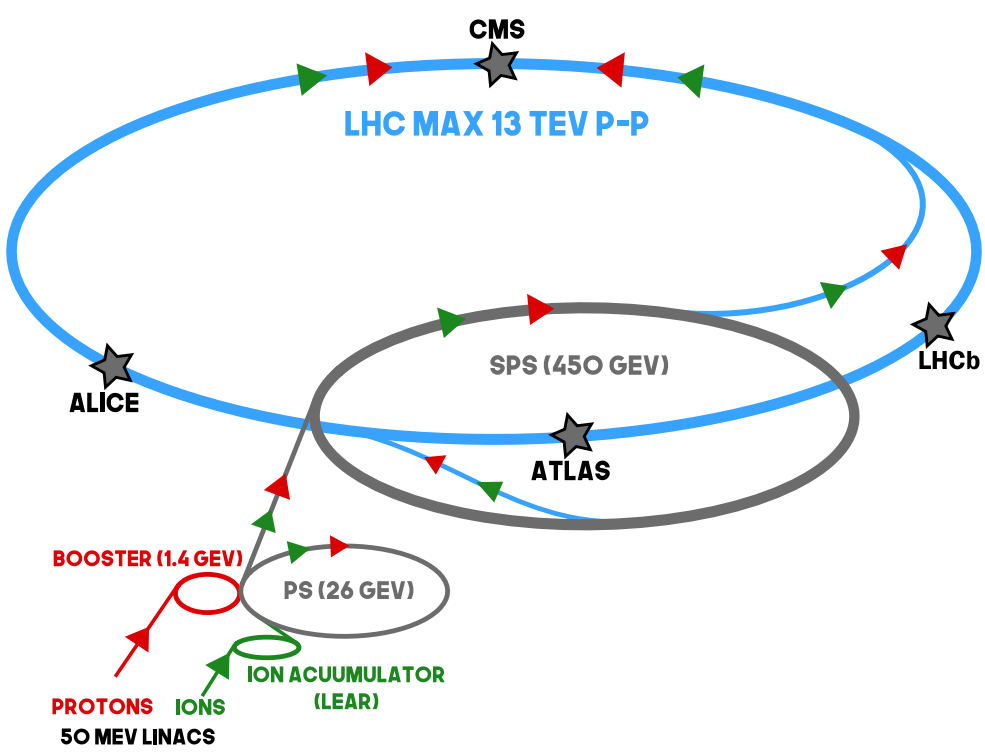

Figure 3.3: LHC injection scheme containing all linear and cyclotron accelerators with their intermediate beam energies and beam directions. Proton Synchrotron (SPS) [117] which further accelerates them to $450 \mathrm{GeV}$ or $176.4 \mathrm{GeV}$ per nucleon, respectively. The SPS has a circumference of $6.9 \mathrm{~km}$ and reached final comissioning in June 1976. In the same year Fermilab also began operation of its Tevatron [118] which exceeded the possible beam energies of SPS by $100 \mathrm{GeV}$ thus taking the crown as most powerful accelerator. During LEP times, the SPS could also accelerate electrons and positrons from 3.5 to $20 \mathrm{GeV}$ which required strong shielding for the magnets to be put in place to protect from the highly directional synchrotron radiation emitted by the leptons. Aside from supplying the previous LEP and current LHC with particle beams, it can also feed fixed target experiments in the SPS West (WA) and North Experimental Area (NA) and also provided proton beams for muon-neutrino experiments in conjunction with the OPERA detector in the Gran Sasso Underground Laboratory in Italy [119].

In the last part of the chain, the particle beams are injected into the LHC in opposing directions in the two beam lines where protons are accelerated up to $6.5 \mathrm{TeV}$ and heavy ions up to $2.5 \mathrm{TeV}$ per nucleon. 


\subsubsection{LHC experiments and physics}

The particle beams of the LHC are brought to collision at predefined interaction points for the four main LHC experiments ALICE, ATLAS, CMS and LHCb. Further smaller experiments focusing on particles produced in forward directions are TOTEM, LHCf and MoEDAL which are located along the beam line on either side of the CMS, ATLAS and LHCb experiment, respectively. A short overview of each experiment except for ALICE and their main physics goals is given in the following.

\section{CMS}

The Compact Muon Solenoid (CMS) [106] is a layered multipurpose detector which gets its name from the comparably compact design with approximately $20 \times 15 \mathrm{~m}^{2}$ in size. In the central detector region it consists of six layers in radial direction which are composed of two silicon detectors, an electromagnetic calorimeter, a hadronic calorimeter, a magnet and lastly a muon detector system. The innermost detector layers are made of silicon pixel detectors spread over three layers ranging from 5 to $10 \mathrm{~cm}$ in radial direction followed by ten layers of silicon strip detectors for 20 to $100 \mathrm{~cm}$. The silicon layers are followed by an electromagnetic calorimeter (ECAL) with projective geometry made of 75,848 lead tungstate crystals of $2.5 \times 2.5 \mathrm{~cm}^{2}$ in size distributed among the central barrel and the end caps. The CMS hadronic sampling calorimeter (HCAL) is made mostly of brass and plastic scintillator layers of $5-8 \mathrm{~cm}$ and $0.3 \mathrm{~cm}$, respectively. It is extended by additional layers located outside of the magnet in order to contain larger hadronic showers. The superconducting magnet surrounding the calorimeters spans a radial direction of 2.9-3.2 $\mathrm{m}$ and generates a strong magnetic field of $4 \mathrm{~T}$. A series of muon detectors is located on the outside of the magnet covering radial distances of 3.5 to $7 \mathrm{~m}$ and additional $5.5-10 \mathrm{~m}$ on the end caps. Figure 3.4 shows a slice through the detector highlighting the detector response to various particles and their trajectory depending on the changing magnetic field. The CMS experiment is focused on the search of various phenomena like supersymmetry (SUSY), extra dimensions and the exploration of unpredicted phenomena. The existence

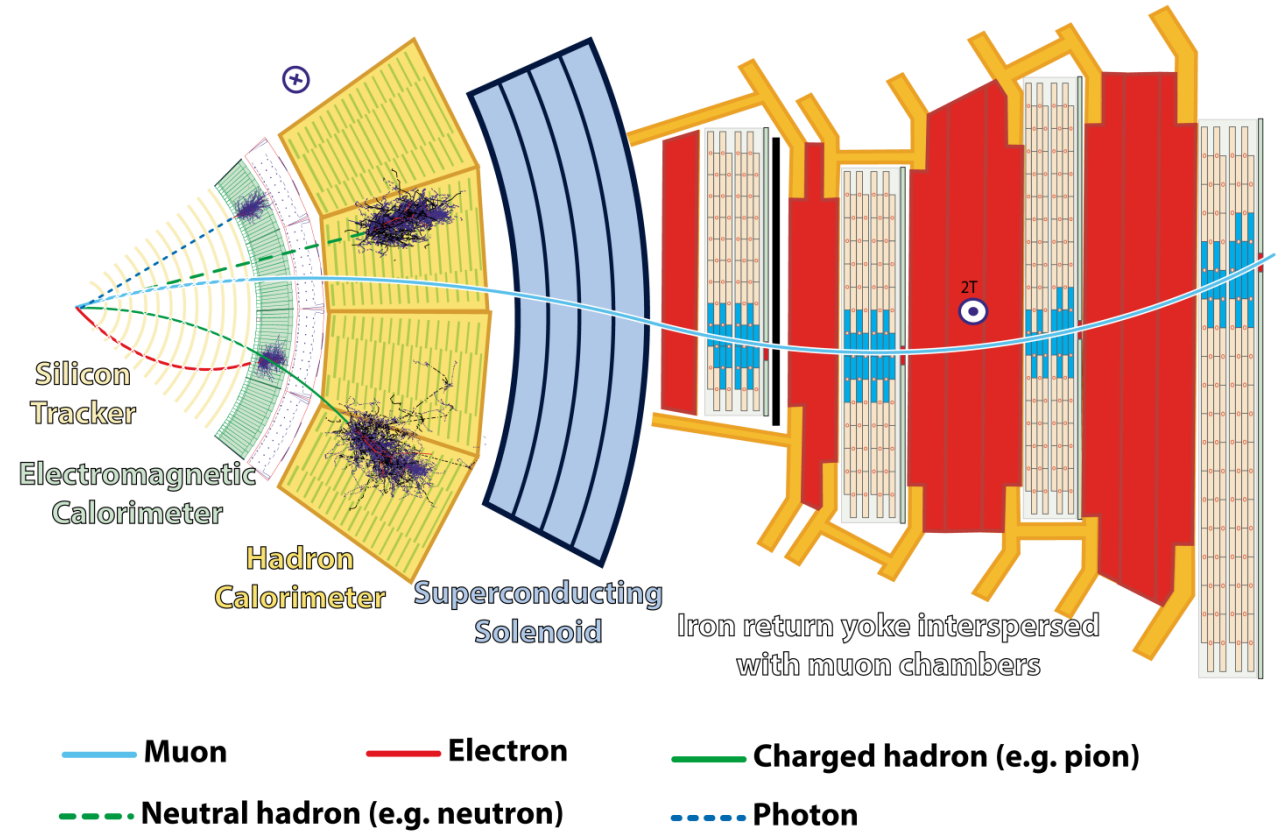

Figure 3.4: Slice of the CMS detector showing the various sub-detectors and their particle hit responses. [120]. 
of SUSY is investigated by searching for particles with opposite spin compared to already known particles like photons or electrons which could help in unifying the electromagnetic, the weak and the strong force. Together with ATLAS, CMS detected the Higgs boson in 2012 confirming theoretical predictions for gauge theories to account for the masses of fundamental particles in the Standard Model.

\section{ATLAS}

The ATLAS (A Toroidal LHC Apparatus) detector [105] is aiming for similar physics as the CMS experiment but with a different detector design focusing more on muon detection. The detector consists of three layers of silicon pixel detectors in radial distances from 5 to 25 $\mathrm{cm}$ followed by additional layers of silicon strip detectors at 30-60 $\mathrm{cm}$ from the interaction point. Different than the CMS detector, ATLAS uses a transition radiation tracking (TRT) and electron identification detector [121] in the next radial layer from 0.6 to $1 \mathrm{~m}$. It is made of 370,000 drift tubes (straws) which can record ionization in the trapped $\mathrm{Xe}-\mathrm{CO}_{2}$ gas mixture in order to track particles traversing the straws. The minimum ionizing tracking signal can be distinguished by the transition radiation induced signal due to the about 5 times larger magnitude of the latter signal. The TRT is surrounded by a solenoid magnet generating a $2 \mathrm{~T}$ magnetic field which is followed in radial direction by electromagnetic and hadronic sampling calorimeters. Similar to CMS, the ATLAS detector has large muon detection systems surrounding the central barrel with additional layers located at its end caps. The distinctive feature of the ATLAS detector is the large barrel toroid magnet that engulfs the muon detector system in a $4 \mathrm{~T}$ magnetic field and gives the experiment its name. ATLAS provided a Higgs measurement in 2012 in the two-photon decay channel in agreement with a measurement by CMS. Its other objectives include topics like SUSY, the search for new heavy bosons, CP-violation measurements and the hunt for new physics.

\section{$\mathrm{LHCb}$}

The LHCb (Large Hadron Collider beauty) experiment [123] follows a different design approach compared to the multipurpose experiments ATLAS and CMS with focus on forward

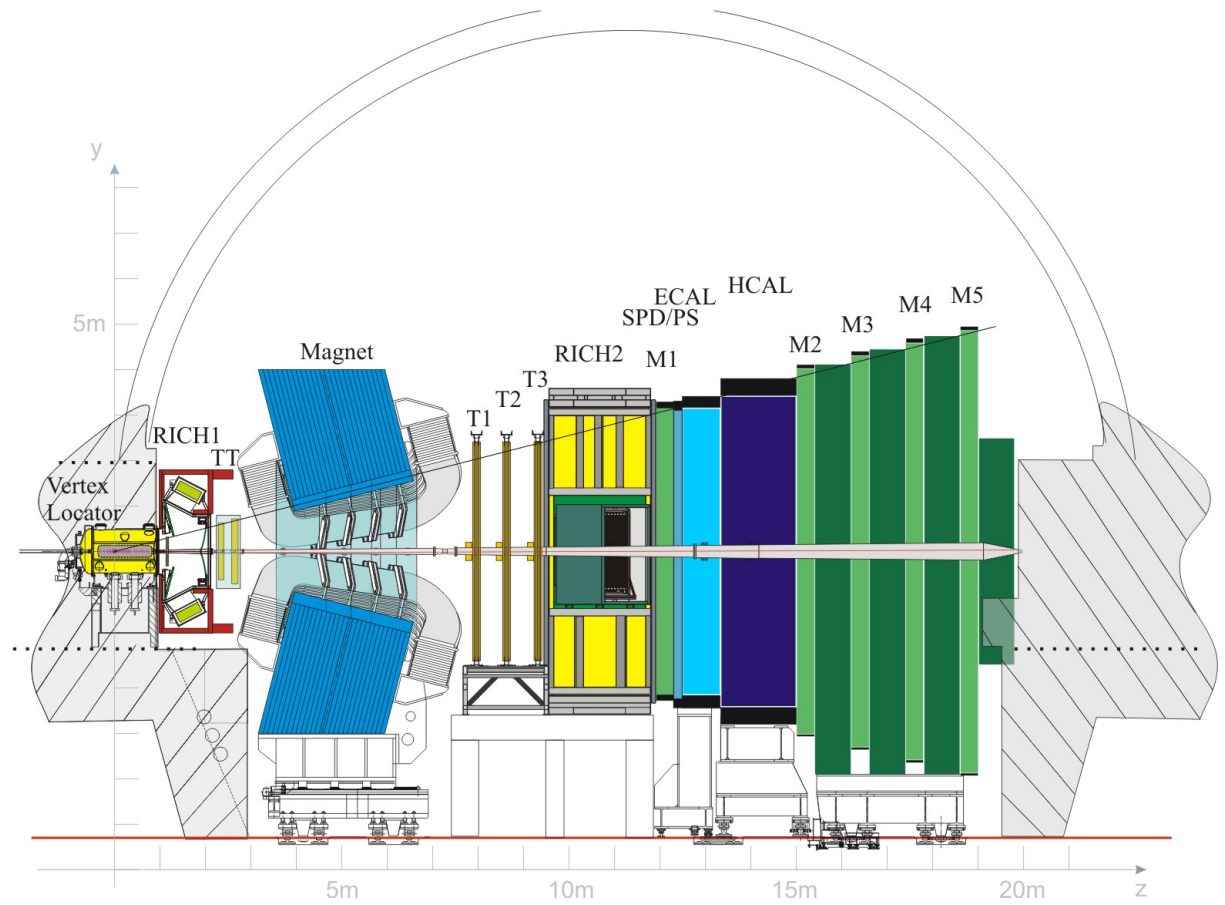

Figure 3.5: Slice of the LHCb detector showing the various sub-detectors. [122]. 
spectrometry. It is designed to measure rare beauty and charm hadron decays as well as CPviolating observables with great precision. As the b-hadrons are predominantly produced in the forward direction, the detector is build as a forward cone to maximize its acceptance for their measurements [124]. Starting at the interaction point, LHCb is equipped with a silicon strip detector called the VELO (Vertex Locator) which, as the name suggests provides the collision vertex location. An interesting design feature of the VELO is the ability to retract it to a safe distance from the beam line during LHC injection where the beams have not reached a stable condition yet. The VELO is followed in forward direction by an array of Ring Imaging Cherenkov (RICH-1) detectors which allows for particle identification via Cherenkov radiation. Cherenkov radiation occurs when a particle traverses a material with a greater speed than the phase velocity of light in this medium. The emitted photons stem from the asymmetric polarization of the material by the traversing charged particle in the $v_{\text {part }}>c / n$ case which causes dipole radiation to occur. This light is emitted under a specific angle $\cos \theta=1 /(n \beta)$ depending on the particle velocity and the refractive index $n$ of the medium allowing for particle identification and momentum measurements. Directly after the RICH another layer of silicon strip detectors is located forming the trigger tracker $(\mathrm{TT})$ which is $1.2 \times 1.5 \mathrm{~m}^{2}$ in size. In order to better distinguish the various particles traversing the $\mathrm{LHCb}$ detector and for momentum measurements of said particles, the TT is followed by the spectrometer magnet generating a strong magnetic field of $4 \mathrm{Tm}$. The main tracking system located shortly after the magnet consists of 12 planes whose parts closest to the beam bike are made of silicon strip detectors while the remaining plane consists of straw tubes for the outer tracker. Remaining particle identification and energy measurements are done using the RICH-2 Cherenkov detector as well as an electromagnetic and a hadronic calorimeter. Lastly the experiment is also equipped with five layers of Multi Wire Proportional Chamber (MWPC) muon detectors together with a selected region of Gas Electron Multiplier (GEM) detectors. A slice view of the LHCb detector visualizing its conical shape and the various sub detector systems in given in Figure 3.5.

\section{Forward experiments}

In forward directions piggybacked to the three large detector systems described above there are additional smaller experiments at the LHC. The TOTEM (TOTal cross section, Elastic scattering and driffraction dissociation Measurement at the LHC) experiment [125] is located within $500 \mathrm{~m}$ of the CMS interaction point and consists of four so-called particle telescopes and 26 Roman pot detectors. It is designed to determine precisely the protonproton interaction cross section and allows for detailed studies of the proton structure itself complementing the measurements in the main LHC detector systems. The MoEDAL (Monopole and Exotics Detector at the LHC) experiment [126] is a forward experiment located in the LHCb cavern aiming to detect magnetic monopoles as well as finding physics beyond the standard model via measurements of highly-ionizing massive long-lived particles. It is comprised of 400 modules of 10 layers each of plastic nuclear-track detectors (NDT) which retain a characteristic damage from the expected monopol particles. Until today the experiment could only provide limits on the monopol production cross section. The LHCf (Large Hadron Collider forward) experiment [127] is made of two sampling and imaging calorimeters located $140 \mathrm{~m}$ from the ATLAS interaction point on either side along the beamline. It aims to measure neutral particles produced in proton-proton collisions in order to further constrain Monte Carlo simulation modeling of cosmic ray interactions. 


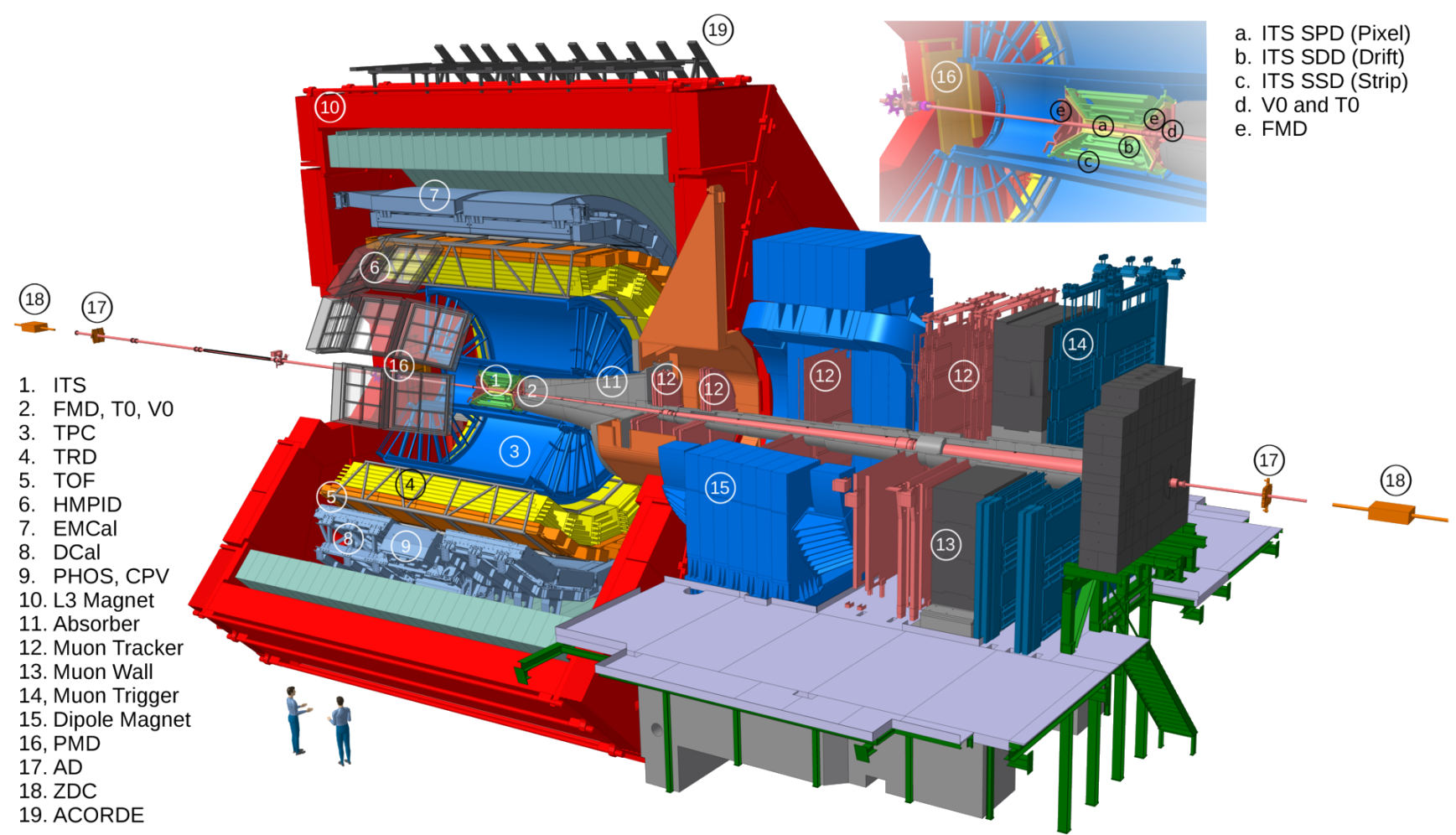

Figure 3.6: The ALICE detector at the CERN LHC in its configuration during the LHC Run 2 data taking periods [129] with an additional zoomed-in depiction of the most central detector systems.

\subsection{A Large Ion Collider Experiment (ALICE)}

The dedicated heavy-ion experiment ALICE [128] is a general-purpose experiment designed to provide particle tracking and identification even in high multiplicity environments of up to $\mathrm{d} N / \mathrm{d} y \approx 8000$ at mid-rapidity. Such conditions can be present in central heavy-ion collisions where ALICE is able to explore the strongly interacting QGP, as described in Section 2.2, and its properties. For this, the experiment focuses on measurements of rare probes which include for example heavy-flavor particles, real and virtual photons, flow measurements, low-mass dilepton studies as well as jet quenching effects. Such measurements require particle tracking as well as PID from very low $p_{\mathrm{T}}$ up to hundredth of $\mathrm{GeV} / c$ for which ALICE is equipped with dedicated subdetector systems. The detector systems can be grouped into two parts depending on whether they are in the central barrel, which includes every system within the red solenoid magnet or whether they are part of the forward muon spectrometer. Figure 3.6 shows a schematic overview of the ALICE detector and its subsystems in the detector configuration used during the LHC Run 2 data taking campaign.

\subsubsection{Detector systems}

The detector systems shown in Figure 3.6 are designed to complement each other in terms of particle identification and momentum reach. They allow to measure and identify a variety of charged and neutral particles from transverse momenta as low as $50 \mathrm{MeV} / c$. The central barrel detector systems are used for hadron and lepton measurements and are therefore embedded into the L3 solenoid magnet [130] which was repurposed from the L3 experiment [131]. The magnet generates a magnetic field of up to $0.5 \mathrm{~T}$ and can switch polarity for data taking and detector calibration. 


\begin{tabular}{|c|c|c|c|c|}
\hline Detector & $\begin{array}{l}\text { ACCEPTANCE } \\
\eta\end{array}$ & $\varphi$ & $\begin{array}{l}\text { RADIAL POS. OR } \\
\text { DISTANCE IP }[\mathrm{cm}]\end{array}$ & Main Purpose \\
\hline \multicolumn{5}{|l|}{ Mid-rapidity } \\
\hline \multicolumn{5}{|l|}{ ITS } \\
\hline SPD & $|\eta|<2(1.4)$ & full & $3.9(7.6)$ & tracking, vertex \\
\hline SDD & $|\eta|<0.9$ & full & $15.0(23.9)$ & tracking, PID \\
\hline SSD & $|\eta|<1$ & full & $38(43)$ & tracking, PID \\
\hline $\mathrm{TPC}$ & $|\eta|<0.9$ & full & $85<r<247$ & tracking, PID \\
\hline TRD & $|\eta|<0.8$ & full* & $290<r<368$ & tracking, $\mathrm{e}^{ \pm}$id \\
\hline TOF & $|\eta|<0.9$ & full* & $370<r<399$ & PID \\
\hline PHOS & $|\eta|<0.12$ & $220^{\circ}<\varphi<320^{\circ}$ & $460<r<478$ & photons \\
\hline EMCal & $|\eta|<0.7$ & $80^{\circ}<\varphi<187^{\circ}$ & $430<r<455$ & photons and jets \\
\hline DCal & $0.22<|\eta|<0.7$ & $260^{\circ}<\varphi<327^{\circ}$ & $430<r<455$ & photons and jets \\
\hline HMPID & $|\eta|<0.6$ & $1.2^{\circ}<\varphi<58.8^{\circ}$ & 490 & PID \\
\hline \multicolumn{5}{|l|}{ Forward } \\
\hline \multirow[t]{2}{*}{ V0 } & $2.8<\eta^{\mathrm{V} 0 \mathrm{~A}}<5.1$ & full & 340 & trigger, centrality, \\
\hline & $-3.7<\eta^{\mathrm{VOC}}<-1.7$ & full & 900 & luminosity \\
\hline \multirow[t]{2}{*}{ T0 } & $4.5<\eta^{\mathrm{T} 0 \mathrm{~A}}<5$ & full & 360 & time, vertex, \\
\hline & $-3.3<\eta^{\mathrm{T} 0 \mathrm{C}}<-2.9$ & full & 70 & trigger \\
\hline PMD & $1.8<\eta<2.6$ & full & 580 & multiplicity \\
\hline \multirow[t]{2}{*}{ FMD } & $1.7<\eta^{\mathrm{FMD} 1,2}<5.0$ & full & $75,83,320$ & multiplicity \\
\hline & $-3.4<\eta^{\mathrm{FMD} 3}<-1.7$ & full & $-75,83$ & \\
\hline
\end{tabular}

Table 1: Different detector systems of the ALICE experiment including their coverage and purpose [132].

As seen in Figure 3.6 and listed in Table 1 the ALICE experiment uses detector systems at mid-rapidity with full azimuthal coverage closest to the interaction point starting with the Inner Tracking System (ITS), followed by a large Time Projection Chamber (TPC), a Transition Radiation Detector (TRD) and lastly a Time-Of-Flight (TOF) detector. Furthermore, there are detector systems with limited acceptance in azimuth and rapidity including the High Momentum Particle Identification Detector (HMPID) and three electromagnetic calorimeters EMCal, DCal and PHOS.

In the following, the different subsystems of ALICE are introduced and their physics performance discussed. In addition, differences between the detector configurations during the LHC Run 1 and Run 2 data taking campaigns are explained.

\subsubsection{Inner Tracking System}

The Inner Tracking System (ITS) [134] of ALICE consists of six cylindrical layers of silicon detectors centered around and closest to the interaction point. It is arranged to cover the full space between the beam pipe and the inner wall of the Time Projection Chamber. The layering consists of two layers of Silicon Pixel Detectors (SPD) at radial position of 3.9 and $7.6 \mathrm{~cm}$, two layers of Silicon Drift Detectors (SDD) at 15 and 23.9 $\mathrm{cm}$ and lastly two layers of Silicon Strip

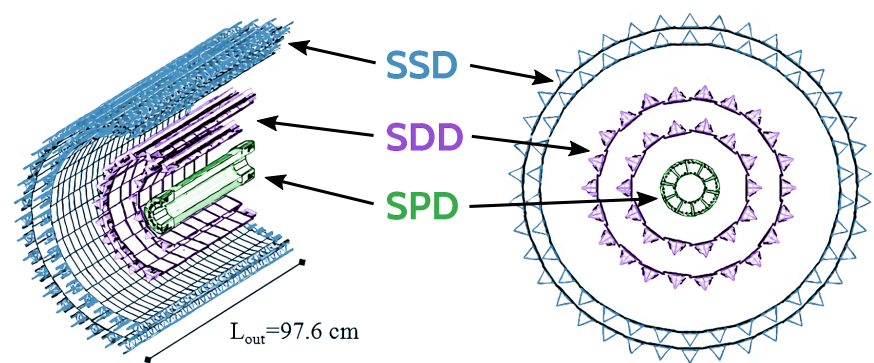

Figure 3.7: Profile view of the ITS and its subsystems. Adapted from Ref. [133]. 
Detectors (SSD) at 38 and $43 \mathrm{~cm}$ with a total of more than 17 million readout channels. The innermost layers furthermore cover $|\eta|<1.75$ in pseudorapidity while the full detector allows tracking for $|\eta|<0.9$. This increased acceptance of the inner layers was chosen to provide multiplicity measurements over a larger range. Illustrative cross section sketches of the ITS are shown in Figure 3.7 showing the radial and longitudinal arrangement of the different layers. The ITS was designed to determine the primary vertex and to separate it from secondary vertices, for example from $\mathrm{D}^{0}$ or $\mathrm{K}_{\mathrm{S}}^{0}$, with a spatial resolution better than $300 \mu \mathrm{m}$ in beam direction and $200 \mu \mathrm{m}$ in the $\varphi-R$ plane. With the close space points of the silicon layers, the ITS can provide tracking even for low $p_{\mathrm{T}}$ particles with $p<200 \mathrm{MeV} / c$. Furthermore, it is used in order to improve the tracking resolution of the TPC by adding additional space points for track reconstruction and allowing to constrain the tracks to the primary vertex. Besides its tracking capabilities, the ITS also provides particle identification at low momentum via energy loss measurements $\mathrm{d} E / \mathrm{d} x$ for which four of the six layers were equipped with analogue readout of a large enough dynamic range. The separation of electrons, pions, kaons and protons can be seen in Figure 3.8 which is feasible for particle momenta $p<1.5$ $\mathrm{GeV} / c$. The ITS was also optimized in terms of material budget in order to keep photon conversions, ionization energy loss as well as multiple scattering to a minimum. The detector represents a radiation length of $0.4 \% X_{0}$ due to the designed overlapping of layers and the incident angles of incoming particles.

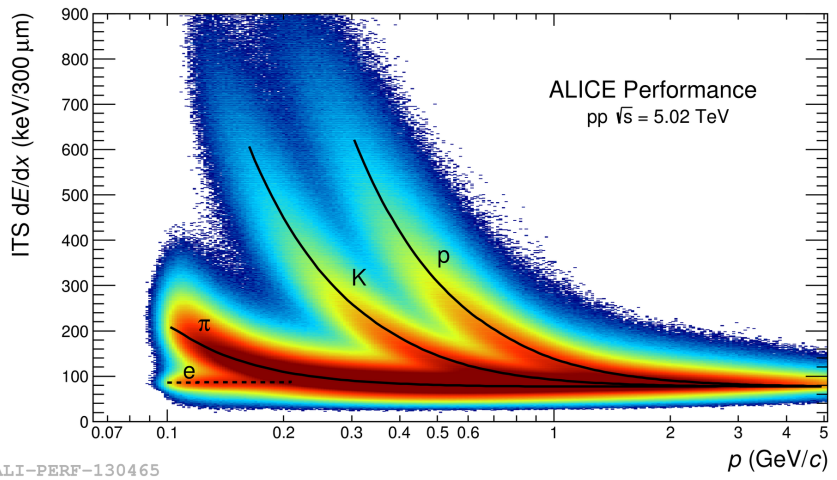

Figure 3.8: ITS-based $\mathrm{d} E / \mathrm{d} x$ energy loss measurement with the SDD and SSD versus combined ITS and TPC reconstructed track momentum in pp collisions at $\sqrt{s}=5.02 \mathrm{TeV}$ [129]. The Bethe-Bloch curves for the expected values are shown in black with the data providing a good separation of the different particle species.

\subsubsection{Time Projection Chamber}

The center piece of the ALICE central barrel is the large Time Projection Chamber (TPC) [135]. With $90 \mathrm{~m}^{3}$, its gas chamber fills a large portion of the L3 magnet and is in return completely covered by the $0.5 \mathrm{~T}$ magnetic field of the magnet. It is intended for tracking of charged particles with good momentum resolution as well as providing the possibility for particle identification via $\mathrm{d} E / \mathrm{d} x$. Furthermore, it must be able to handle charged particle densities up to $\mathrm{d} N_{\mathrm{ch}} / \mathrm{d} \eta=8000$ to deal with the high multiplicity environments of central $\mathrm{Pb}-\mathrm{Pb}$ collisions at interaction rates of about $8 \mathrm{kHz}$.

The TPC is designed with a total of $32.5 \mathrm{~m}^{2}$ readout pads at the end caps and a cylindrical field cage with a central electrode. Between the end caps and the central electrode a strong electric field is applied which leads to typical drift times of tens of $\mu$ s for the ionized particles to reach the readout region. The TPC covers radial distances from $84.8 \mathrm{~cm}$ up to $246.6 \mathrm{~cm}$ with respect to the beam pipe and wraps around the beam pipe in full azimuth over a length of $500 \mathrm{~cm}$ in beam direction. The dimensions of the TPC in $z$-direction allow for tracking over a pseudorapidity range of $|\eta|<0.9$ with the inner and outer readout pads. When using only the inner tracking part of the TPC a coverage of $|\eta|<1.4$ is possible.

The initial gas in the central barrel was chosen to be a $90 \% \mathrm{Ne}$ and $10 \% \mathrm{CO}_{2}$ mixture which followed studies at the NA49 [136] experiment. This mixture was modified during the Run 2 data taking campaign to an initial use of Nitrogen and a later transition to Argon instead of 
Neon in 2016 and 2018. This change was necessary in order to reduce the space charge buildup and the resulting tracking distortions in the TPC. The field cage with its common high-voltage electrode at $z=0$ provides an electric drift field of up to $400 \mathrm{~V} / \mathrm{cm}$ which therefore requires the electrode to be at a voltage of $100 \mathrm{kV}$ in order to allow for the drift over $2.5 \mathrm{~m}$. The readout planes at the TPC endcaps are segmented into 18 trapezoidal slices as well as an inner layer and outer layer. The design was optimized for minimal dead areas in both azimuthal and radial direction with only about $3 \mathrm{~cm}$ of dead area in azimuthal direction of which the pad frame is the dominant part. The signal itself is recorded via readout chambers consisting of one wire layer of a gating grid to reduce electron backflow, one layer of cathode wires and one layer of anode wires which are spaced $2.5 \mathrm{~mm}$ apart followed by multiple readout pads behind the wire grid.

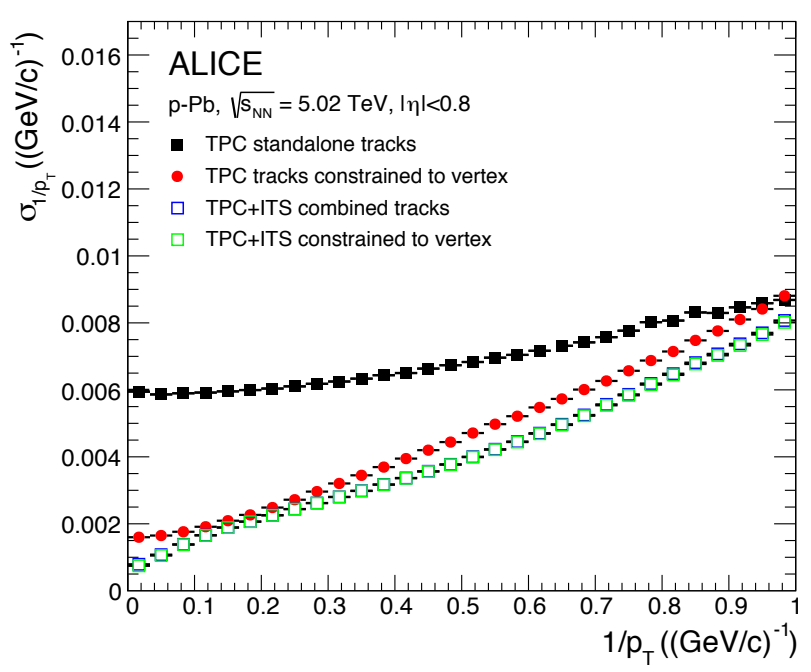

Figure 3.9: Transverse momentum resolution for tracking with standalone TPC and matched tracking of ITS-TPC which can further be constrained to the primary vertex. The constraint on the primary vertex is only relevant for TPC standalone tracks [137]. ing distance from the interaction point measuring $4 \times 7.5 \mathrm{~mm}^{2}$ in the inner chambers and $6 \times 15$ $\mathrm{mm}^{2}$ in the outer most chambers. In total, the TPC uses 570312 readout pads over both endcaps. For particle tracking the TPC uses the combined measurement of drift time towards the readout pads and the $x-y$-plane position of the readout pad signal. With this information, a 3-dimensional tracking in the large gas volume is possible and the transverse momentum of charged tracks due to the bending in the magnetic field can be determined. The $p_{\mathrm{T}}$ resolution of the TPC tracking is depicted in Figure 3.9 showing a sub-percent momentum resolution for $p_{\mathrm{T}}>1 \mathrm{GeV} / c$ TPC standalone tracks [137]. The tracking performance is further improved if the tracks are constrained to the vertex or if ITS-TPC track matching is performed.

Aside from the particle tracking, the TPC also provides particle identification via energy loss measurements in the gas. This energy loss signal originates from electromagnetic interactions of the charged particles with the gas which causes the gas in the particle trail to ionize. The energy loss from such interactions is usually small compared to the particle energy which causes the particles in most cases to not be stopped in the gas. The Bethe-Bloch-equation $[139,140]$ is

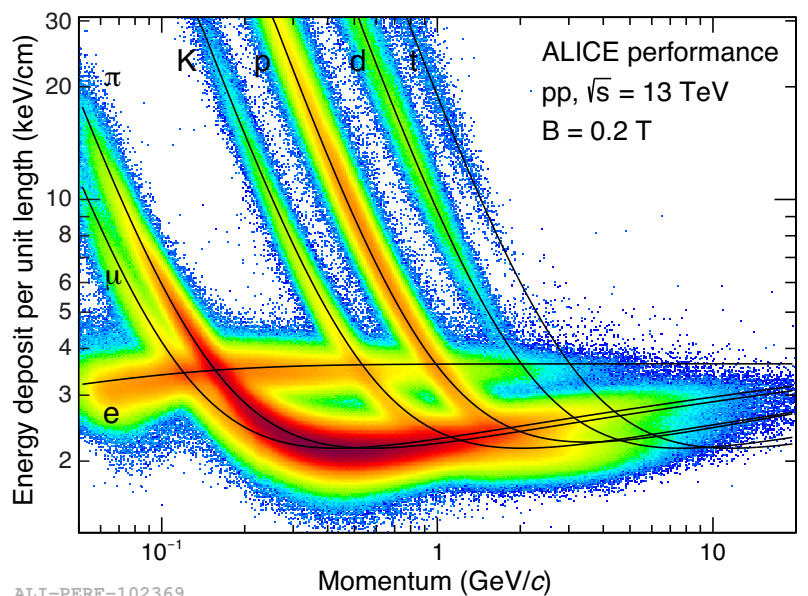

Figure 3.10: Energy loss signal $\mathrm{d} E / \mathrm{d} x$ versus the particle momentum $p$ for pp collision data at $\sqrt{s}=13 \mathrm{TeV}$ with a low magnetic field [138]. The Bethe-Bloch lines according to Equation 12 are shown for the different identified particle species as black lines. 
used to describe the mean energy loss signal per path length $\mathrm{d} E / \mathrm{d} x$ at intermediate energies via

$$
-\frac{\mathrm{d} E}{\mathrm{~d} x}=2 \pi N_{a} r_{e}^{2} m_{e} c^{2} \rho \frac{Z}{A} \frac{z^{2}}{\beta^{2}}\left[\ln \left(\frac{2 m_{e} \gamma^{2} v^{2} T_{\max }}{I^{2}}\right)-2 \beta^{2}-\delta 2 \frac{C}{Z}\right],
$$

where the atomic mass numbers of the medium are given by $Z$ and $A$ and the particle charge and velocity by $z$ and $v$, respectively. Further quantities are the Avogadro number $N_{a}$ and the mean excitation potential $I$ which is approximately $I=10 \cdot Z \mathrm{eV}$ [141].

Figure 3.10 shows the distribution of the TPC $\mathrm{d} E / \mathrm{d} x$ energy loss signal in pp collision data at $\sqrt{s}=13 \mathrm{TeV}$ with a low magnetic field setting of $0.2 \mathrm{~T}$ [138]. A clear separation of the energy loss signal from different particle species can be seen up to intermediate momenta. Up to $p \approx 4 \mathrm{GeV}$ the heavier charged particles, namely kaons, protons, deuterons and tritons are significantly separated and follow their Bethe-Bloch energy loss prediction. For high momentum, the different energy loss bands start to merge with each other making a separation of the particle species difficult. The TPC achieves a resolution of the energy loss signal based on cosmic tracks of $5-9 \%$ with better resolution for tracks with a higher number of available clusters.

\subsubsection{Transition Radiation Detector}

The ALICE Transition Radiation Detector (TRD) [142] is a six layered multi-wire proportional chamber (MWPC) detector at a radial distance of $2.9<R<3.7 \mathrm{~m}$ from the interaction point. It consists of 18 super-modules which are divided into five sectors in $z$ direction and covers full azimuth as well as $|\eta|<0.9$. Each detector element is made of a $48 \mathrm{~mm}$ thick radiator, a 30 $\mathrm{mm}$ long drift section followed by a $7 \mathrm{~mm}$ MWPC with readout pads. The drift region is filled with a $85 \%$ xenon and $15 \% \mathrm{CO}_{2}$ gas mixture to allow for a controlled ionization. The detector was designed to extend the tracking capabilities of the TPC at high transverse momentum and to reject charged pions and identify electrons over a large $p_{\mathrm{T}}$ range. The latter is achieved with transition radiation emitted by particles with a Lorentz factor $\gamma>1000$ in the alternating fiber and foam radiator layer in front of the TRD. Transition radiation has been predicted in 1946 and can occur when a charged particle crosses material boundaries in an inhomogeneous material [143]. The incident particles are required to be above the production threshold of $\gamma \approx 1000$ in order to produce transition radiation photons. The probability for the radiation of photons from this effect is small and thus requires a multitude of boundaries to cross which is realized for the TRD in the form of a fibre-foam sandwich material. Approximately 1.45 photons with energies of $1-$ $30 \mathrm{keV}$ are produced via transition radiation of an electron. Photons of such low energy convert within the first centimeter of the drift gas and the resulting electrons drift towards the readout area where they are amplified and detected. Electrons can be distinguished from charged pions in the TRD via the drift time and signal strength as shown in Figure 3.11 where a lower overall signal for pions is observed due to the lower specific energy loss at

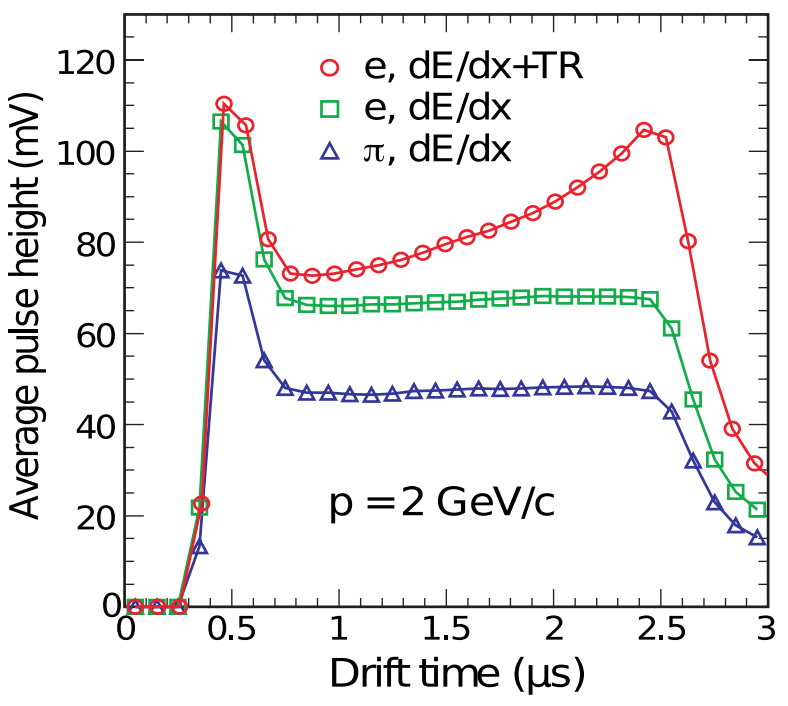

Figure 3.11: TRD pulse signal versus drift time for pions (blue), electrons without a radiator (green) and electrons with a radiator $(r e d)$ for a momentum of $2 \mathrm{GeV} / c$ [128]. the same momentum. 
Furthermore, at large drift times an additional signal for electrons is observed due to the early conversions of the transition radiation photons which will appear late due to the drift time in the readout pads [128]. A further use of the TRD is its tracking capability and the improvement of position and momentum resolution when combining its information with the TPC tracking. The TRD standalone tracking provides a momentum resolution of $\delta p_{\mathrm{T}} \approx 2.5-3 \%$ while the combined central barrel tracking including TRD can reach a resolution of less than $5 \%$ up to $100 \mathrm{GeV} / c$. In addition, the TRD can also provide Level 1 event triggers in order to enhance statistics for high- $p_{\mathrm{T}}$ electrons, heavy particles and jet measurements. The trigger employs the Global Tracking Unit (GTU) which inspects TRD tracklets during data taking and returns a trigger signal within $6.1 \mu$ s after the collision. A very important feature provided by the TRD and its tracking is the ability to correct TPC space points used for tracking. This feature is important as the high occupancies in high luminosity or high multiplicity events lead to an accumulation of positively charged ions in the TPC as they are about a factor 1000 slower than electrons. Such accumulations distort the otherwise uniform drift field and therefore cause the track space points to be shifted with respect to their actual position by up to several centimeters. The correction for these distortions is done by reconstructing tracks with combined ITS-TPC-TRDTOF tracking and comparing the resulting reference track points in the TPC with the distorted points to obtain correction factors. A visualization of what a distorted track looks like is given in Figure 3.12 with the distorted TPC track shown in blue, the combined central barrel track in red and the deduced reference track points in the TPC in green.

\subsubsection{Time of Flight Detector}

The ALICE TOF [145] detector is a cylindrical detector located about $3.7 \mathrm{~m}$ from the interaction point covering $|\eta|<0.9$ in pseudorapidity and the full azimuth. In order to reduce the material budget in front of PHOS, the modules in front of the calorimeter were left out. The TOF is designed to separate signals from pions, kaons and protons at intermediate $p_{\mathrm{T}}$ where the TPC and ITS $\mathrm{d} E / \mathrm{d} x$ energy loss signals can no longer be used as efficient discriminators. It furthermore provides electron identification at low momentum. For time-of-flight measurements, TOF uses 1593 Multigap Resistive Plate Chamber (MRPC) strip detectors spread over its acceptance and divided into 18 sectors. The strips were found to provide a time resolution better than $50 \mathrm{ps}$ in a test beam

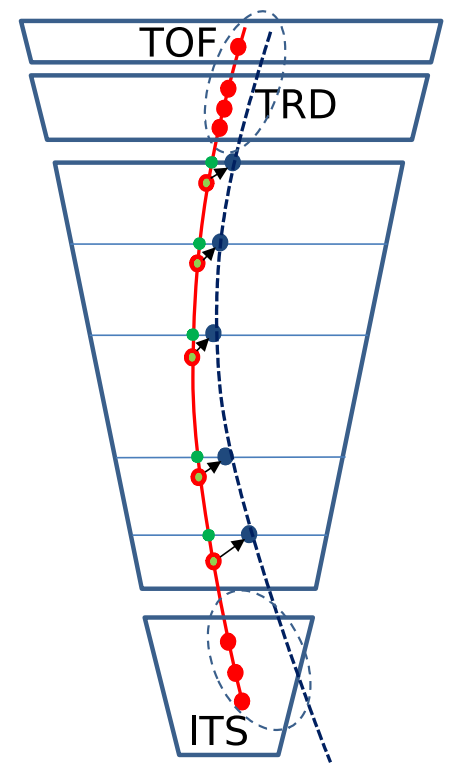

Figure 3.12: Distortion correction approach with combined ITS-TPC-TRDTOF information [144]. The actual TPC track space points (red) are compared to the distorted points (blue) providing reference points (green).

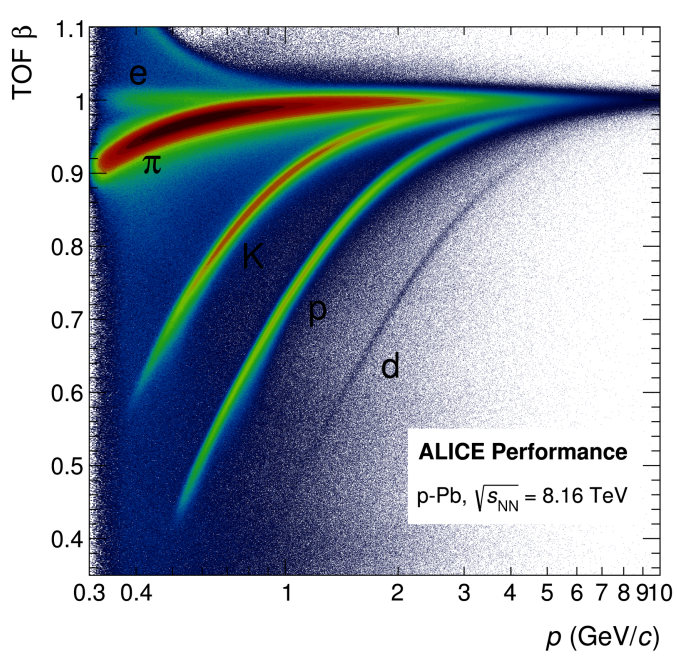

Figure 3.13: TOF $\beta$ versus track momentum in $\mathrm{p}-\mathrm{Pb}$ collision data at $\sqrt{s_{\mathrm{NN}}}=8.16$ $\mathrm{TeV}$ [129]. 


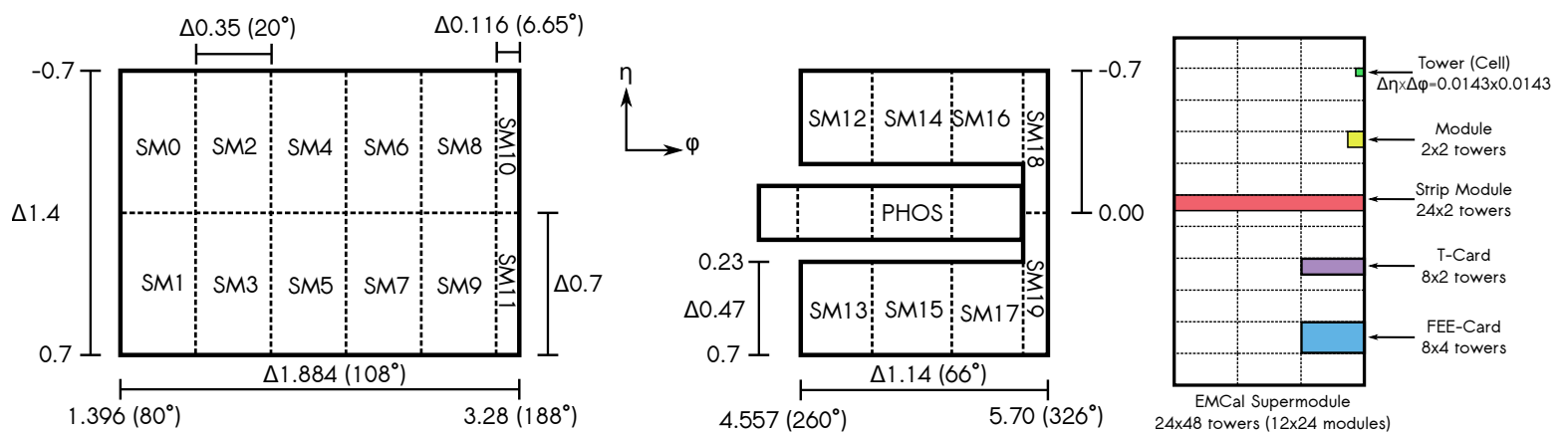

Figure 3.14: Left: Geometric overview of the EMCal and DCal detectors in the $\eta$ - $\phi$ plane. The full LHC Run 2 setup is shown with all 20 supermodules (SM0-SM19) as well as the PHOS detector in the DCal gap. The supermodule numbering scheme is the one used in EMCal offline treatment. Right: Zoom on a single EMCal supermodule in the $\eta$ - $\phi$ plane including visualizations of sub-components and their tower coverage.

setup [146]. Shown in Figure 3.13 is the TOF time of flight signal $\beta$ versus the incident particle momentum in $\mathrm{p}-\mathrm{Pb}$ collision data at $\sqrt{s_{\mathrm{NN}}}=8.16 \mathrm{TeV}$ [129]. A clear separation of various particle species is visible up to large momenta, especially for heavy particles where a separation of pions, kaons and protons better than $3 \sigma$ is achieved $[132,147]$.

\subsubsection{Electromagnetic Calorimeters EMCal and DCal}

The ALICE EMCal [148] was designed with a focus on jet quenching measurements where it serves as an event trigger and allows to measure the neutral jet components with high precision. Furthermore, it extends the $p_{\mathrm{T}}$ reach of the ALICE detector systems for electromagnetic probe measurements like photons and electrons. The detector is a large lead-scintillator sampling calorimeter about $R \approx 4.5$ $\mathrm{m}$ from the interaction point. It has a cylindrical geometry covering $|\eta|<0.7$ in pseudorapidity and $\Delta \varphi=107^{\circ}$ in azimuth and is located opposite of the PHOS detector. A visualization of the EMCal geometry in the ALICE central barrel is given in Figure 3.15. With the prospect of enabling di-jet analyses, the EMCal was extended in 2015 by the Di-jet Calorimeter (DCal) [149]. The DCal is located opposite in azimuth around the PHOS detector and covers $0.22<|\eta|<0.7$ for $260^{\circ}<\varphi<320^{\circ}$ and $|\eta|<0.7$ for $320^{\circ}<\varphi<327^{\circ}$ adding six $2 / 3$ and two $1 / 3$ supermodules to the overall calorimeter acceptance. The additional modules are based on the same hardware as EMCal with respect to cell design, detector readout as well as triggering. Figure 3.14 shows the $\eta-\varphi$ projection of the EMCal and DCal and the different full supermodules with $\Delta \varphi=20^{\circ}$

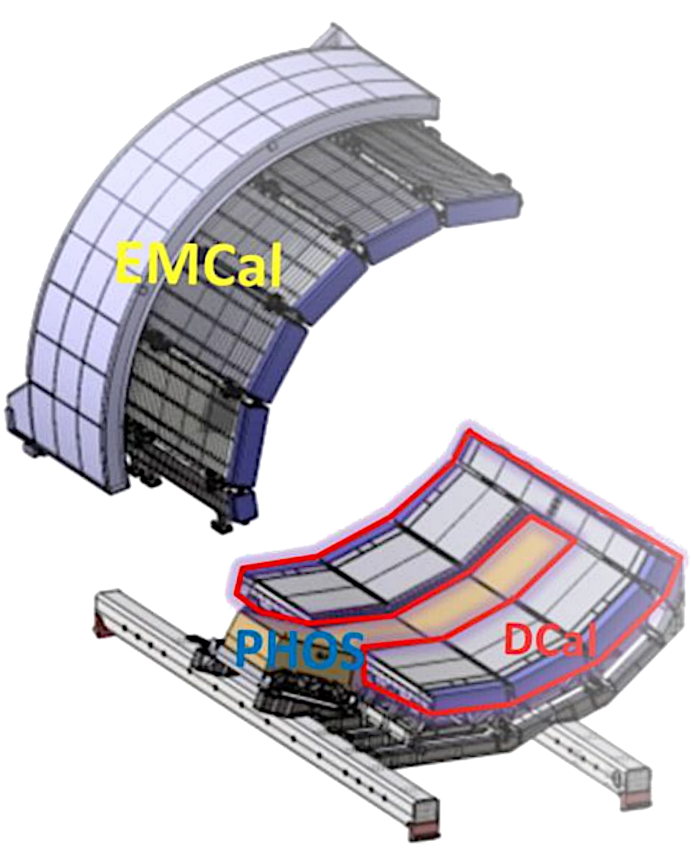

Figure 3.15: Visualization of the 3D geometry of the EMCal and DCal together with the PHOS detector. [148]. in azimuth, the $2 / 3$ supermodules of DCal and the $1 / 3$ supermodules that are located at the higher end 
in azimuth. Supermodules are sub divided into modules of $2 \times 2$ cells with are mounted projective to the interaction point and amount to a total of 12,288 cells for EMCal and 5,376 cells for DCal. Both detectors are segmented in azimuth similar to the TPC, TRD and TOF so that the borders between individual supermodules overlap with the TPC sector boundaries in order to make place for supporting structures in a region where readout is not necessary.

The individual cells of the calorimeter measure $\sim 6 \times 6 \mathrm{~cm}^{2}$ or $\Delta \eta \times \Delta \varphi=0.0143 \times 0.0143 \mathrm{rad}^{2}$ and are made of alternating layers of lead and polystyrene scintillator amounting to approximately 20.1 radiation lengths. With these dimensions and the used material, this corresponds to approximately two times the Molière radius. The scintillation photons are captured by wavelengthshifting fibers which run longitudinally through the cell and terminate on an avalanche photo diode (APD) with an active area of $5 \times 5 \mathrm{~mm}^{2}$. The EMCal has an energy resolution better than 5\% for high energetic clusters as shown in Figure 3.16 while for lower energies it worsens to about $14 \%$. The resolution was determined from a test beam setup where an EMCal test module with $8 \times 8$ cells was tested using particle beams (hadrons, electrons and muons) from the Proton Synchrotron and the Super Proton Synchrotron at CERN at beam energies of 0.5 $\mathrm{GeV}$ to $250 \mathrm{GeV}$. In order to track the particle beam hit position, MWPCs were placed in front of EMCal for exact position determination. From the testbeam data also the nonlinearity of the detector response as well as the dependence on the cell hit position was determined via comparisons of the $\mathrm{N} \times \mathrm{N}$ clusterizer reconstructed cluster energies in data and simulation as shown in Section 5.2.3. This nonlinearity correction is followed by a cell energy calibration where all EMCal and DCal cells are shifted to the same response via reconstruction of $\pi^{0} \rightarrow \gamma \gamma$ decays requiring each individual cell as the leading cell for one of the photon clusters. Some cells show a problematic energy response where they either do not register hits or they show a noisy or cold (lower than average energy spectrum) behavior. Such cells are excluded on analysis level via a bad channel masking procedure where the time and energy distributions of each cell are compared to an expected average of good cells. This procedure masks approximately $6-9 \%$ of all channels in the calorimeter with variations depending on the data taking conditions where also full front-end (FE) cards (see Figure 3.14 can become noisy and be masked.

Another part of the calibration is related to variations in the EMCal supermodule temperatures which can also depend on the current season during data taking. For the EMCal temperature variations of up to $4{ }^{\circ} \mathrm{C}$ from the average of $24.5{ }^{\circ} \mathrm{C}$ are seen while for the DCal due to the proximity to the strongly cooled PHOS detector the variations are less than $1.2{ }^{\circ} \mathrm{C}$ with an average of 20 ${ }^{\circ} \mathrm{C}$. The correction for the temperature dependent gain difference of the APDs is based on fixed signals induced by an LED light system. As the LED system is set to a specific amplitude and wavelength, the readout of this signal and its change due to the temperature change ( $\left.L E D_{\text {average }} / \mathrm{LED}_{\text {meas }}\right)$ can be parametrized with a linear function depending on the temperature resulting in an up to $5 \%$ correction factor. The final correction for EMCal cells is for the timing information and its shift due to the cable length of the readout

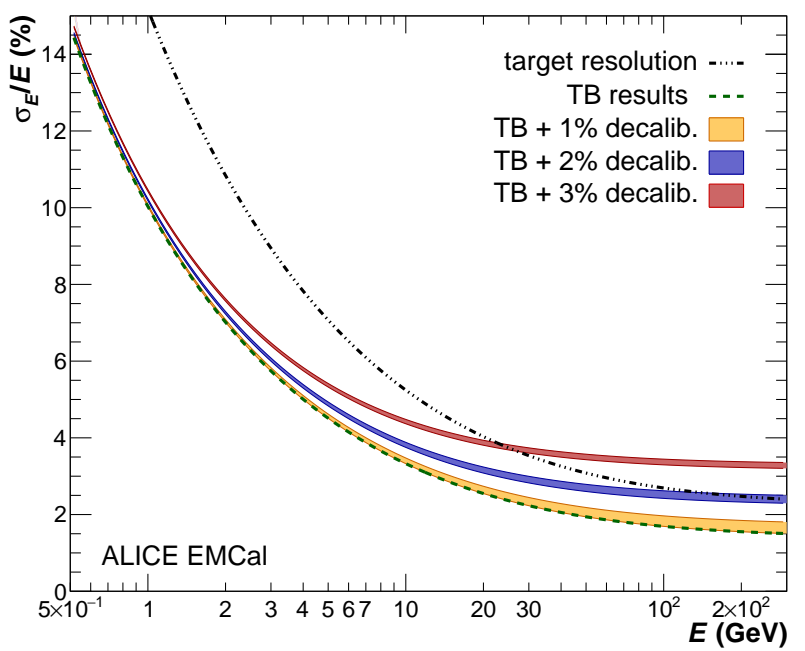

Figure 3.16: Relative energy resolution versus particle energy for the intended target resolution (black) and the resolution obtained from the test beam (green) [150]. 
system (around $600 \mathrm{~ns}$ ). Furthermore, it includes the correction for the shift of the internal EMCal clock of the ALTROs and the LHC clock which is determined on a run-by-run basis. For the time calibration, the time signal of each cell is shifted to the optimum value of 0 ns based on the average position of the cell signal obtained the mean value of the distribution only including cell signals with amplitudes of more than $400 \mathrm{MeV}$. This leads to a timing resolution of about 1.4-4 ns with better values for higher energies.

The EMCal is in addition used as an event trigger with focus on photon and jet signal enhancement. Its triggers have to be in coincidence with a minimum bias trigger and can be at level-0 (L0) and at level-1 (L1) which allows for a different amount of processing time of $1.2 \mu \mathrm{s}$ and $6.5 \mu \mathrm{s}$, respectively. The L0 trigger is based on the energy sum above a given threshold in a sliding $4 \times 4$ cell window within one Trigger Region Unit (TRU) which by itself covers $8 \times 48$ cells. The trigger is named depending on the underlying minimum bias event trigger as EMC1, EMC7 or EMC8. As the INT7 event trigger, see Section 3.2.1.8, is used in this analysis, the L0 trigger is referred to as EMC7 in the following. Given the additional time available for the L1 trigger signal, the EMCal L1 trigger can further process its signals by comparing multiple TRU signals within a Summary Trigger Unit (STU). This can provide designated photon triggers (EGA) based on a $4 \times 4$ cell sliding window which can now also cross TRU borders to increase the acceptance of the trigger. These triggers can be set to different thresholds and are referred to as EG2 and EG1 for the low and high threshold trigger, respectively. Further L1 triggers with focus on jet signal enhancement use energy sums over larger areas of $16 \times 16$ or $32 \times 32$ cells and are referred to EJ2 and EJ1 depending on their threshold.

\subsubsection{Photon Spectrometer PHOS}

The PHOS [151] is a high granularity electromagnetic calorimeter located in the central barrel at a radial position of $460 \mathrm{~cm}$ from the IP and covering $|\eta|<0.125$ in pseudorapidity and $70^{\circ}$ in azimuth. Its position relative to the EMCal and DCal is shown in Figure 3.15. The detector was designed to cover direct photon and neutral meson measurements from very low to high $p_{\mathrm{T}}$ in order to study these important probes of the QGP. The calorimeter is comprised of three full and one half module consisting of a total of 12544 cells made of lead-tungstate $\left(\mathrm{PbWO}_{4}\right)$ crystals with a size of $22 \times 22 \mathrm{~mm}^{2}$ each. It has a good energy resolution ranging from about $5.5 \%$ at $500 \mathrm{MeV}$ to $1.2 \%$ at $100 \mathrm{GeV}$ cluster energies due to the small Molière radius of $2 \mathrm{~cm}$ of the cells in combination with a high light yield.

The energy resolution as a function of the electron test beam energy from the CERN PS and SPS is shown in Figure 3.17 for the energy range $0.5<E_{\text {beam }}<150 \mathrm{GeV}[152]$. During the LHC Run 1 data taking campaign only three modules of the PHOS were installed and the additional half module was added in 2015. In order to reduce background in the calorimeter, it is cooled to $-25{ }^{\circ} \mathrm{C}$. In addition the TRD and TOF modules in front of the calorimeter were removed to keep the material budget to only $0.2 X_{0}$ thus reducing the energy loss from photon conversions. The PHOS is furthermore equipped with a charged particle veto (CPV) detector which allows to distinguish between charged and neutral clusters. This detector covers in its current configura-

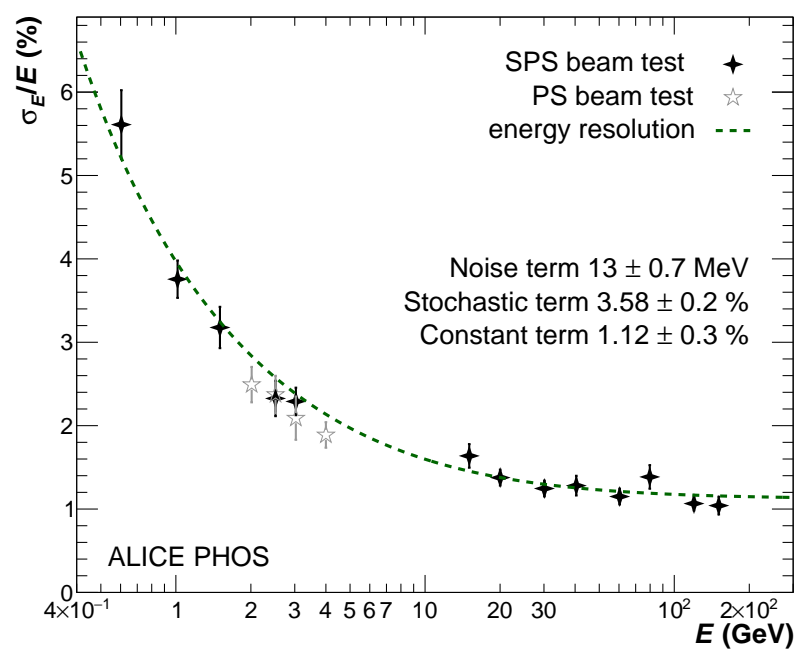

Figure 3.17: Average energy resolution of the PHOS versus SPS and PS electron beam energy together with the resolution function [152]. 
tion one module of the PHOS and provides a track position resolution of 3-4 mm while detecting close to all charged tracks in its acceptance [153]. The CPV itself is based on MWPC technology with an Ar- $\mathrm{CO}_{2}$ gas mixture and cathode readout pads of $22 \times 11 \mathrm{~mm}^{2}$. It is installed only $5 \mathrm{~mm}$ above the PHOS for the best possible charged track hit extrapolation. The PHOS can provide an event trigger at Level-0 based on the energy deposit in a sliding $4 \times 4$ cell array within one TRU region $(28 \times 16$ cells) [154]. Additional triggers at L1 are available with three different thresholds above the L0 threshold. All triggers aims to enhance statistics for photon analyses at high $p_{\mathrm{T}}$.

\subsubsection{High Momentum Particle Identification Detector (HMPID)}

The ALICE HMPID [155] consists of seven $1.5 \times 1.5 \mathrm{~m}^{2}$ large modules of proximity-focusing Ring Imaging Cherenkov (RICH) counters about $4.7 \mathrm{~m}$ from the interaction point covering $|\eta|<0.6$ in pseudorapidity and $57.6^{\circ}$ in azimuth. The detector is built projective on the interaction point and serves for measurements of charged particles where it extends the PID capabilities of ALICE to high momentum. It provides a separation of $3 \sigma$ for pions and kaons at $p \approx 1-3 \mathrm{GeV} / c$ as well as for $\mathrm{K} / \mathrm{p}$ in the momentum region $p<5 \mathrm{GeV} / c$.

\subsubsection{Forward Detectors}

ALICE is equipped with a variety of detectors in the forward (and backward) region. These detectors provide not only the minimum bias L0 event triggers but also the charged particle multiplicity and centrality in heavy ion collisions. Furthermore, they are used to determine the collision time and the event reaction plane. In the following, a short overview of the forward detectors is given:

The Zero Degree Calorimeter (ZDC) [156] is designed to determine the centrality of heavy ion collisions by determining the number of non-interacting nucleons (called spectators). The detector is divided into three separate subsystems which focus on the measurement of a different particle species due to the spatial separation of protons and neutrons in the magnetic elements of the LHC. For neutron detection the ZDC is equipped with a neutron calorimeter (ZN) between the LHC beam lines and for proton detection is uses its proton calorimeter (ZP) which is located on the side of the LHC beam pipe where positive particles are deflected to. An additional electromagnetic calorimeter about $7 \mathrm{~m}$ from the interaction point allows to measure mainly $\pi^{0}$ meson decay photon energy to help distinguish central and peripheral collisions.

The Photon Multiplicity Detector (PMD) [157] allows to measure the photon multiplicity as well as their spatial distribution for an event plane determination. The detector uses the preshower method to measure photon signals from electromagnetic showers induced in a three radiation length thick converter and read out by gas proportional chambers. An additional counter is installed in front of the converter to serve as a charged particle veto (CPV). With its photon measurements the PMD also allows for studies of azimuthal anisotropy and flow.

The Forward Multiplicity Detector (FMD) [158] is an additional detector system in ALICE that can provide charged particle multiplicity measurements as well as an event plane determination. Its five rings of silicon strip detectors are located around $60-320 \mathrm{~cm}$ from the interaction point and cover the combined forward regions of $-3.4<\eta<-1.7$ and $1.7<\eta<5.0$.

The V0 detector [159] is made of two arrays of scintillation counters that are placed on either side of the interaction point along the beam line at pseudorapidities of $2.8<\eta<5.1$ (V0A) and $-3.7<\eta<-1.7$ (V0B). The detector provides a fast minimum bias event trigger which 

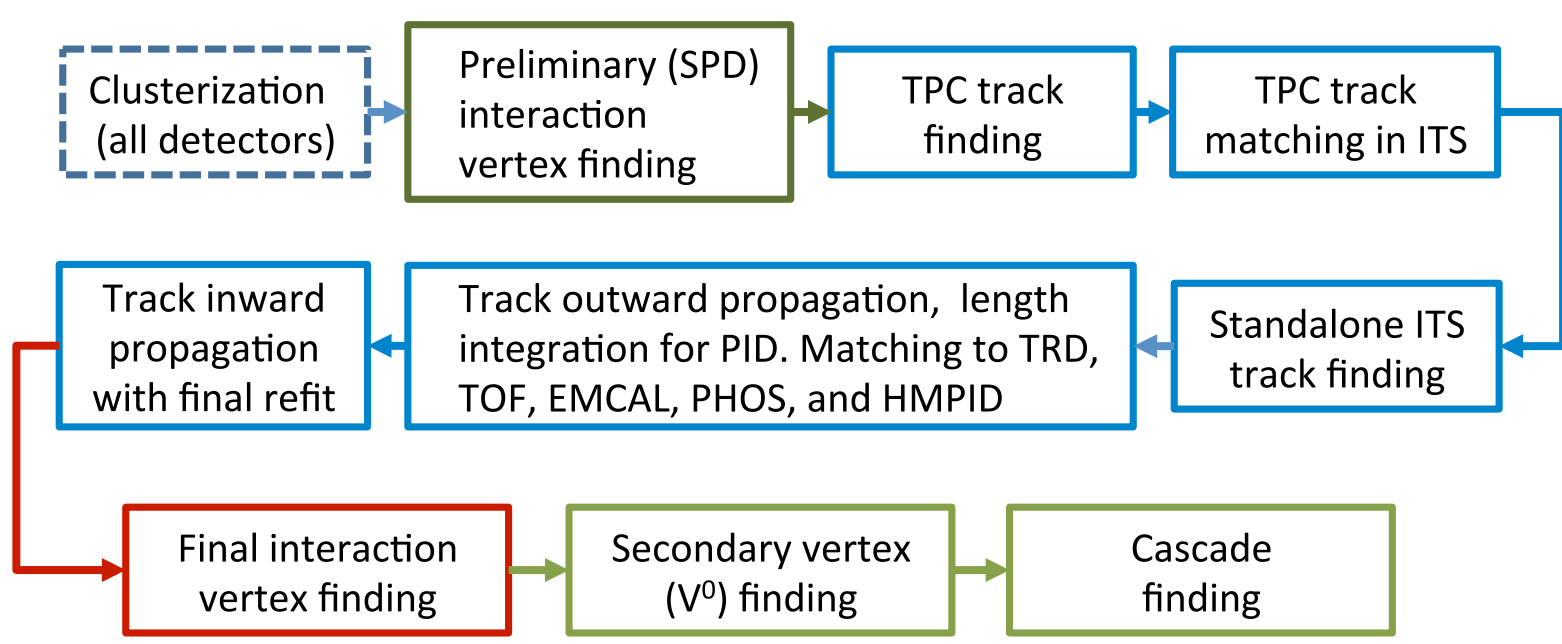

Figure 3.18: Reconstruction flow in ALICE from raw data to tracks and vertices. Taken from Ref. [137].

can operate in a coincidence mode (V0AND or INT7) of signals in both V0 arrays or in a mode requiring a signal in at least one of the arrays (V0OR or INT1). The V0AND trigger operates with an efficiency of about 75-84 \% in pp collisions providing events with at least two charged particles [160]. Due to a correlation between the V0 signals and the number of actual primary particles in a collision, the V0 furthermore can help determine the event multiplicity and therefore the centrality of heavy-ion collisions. The detector is also crucial in van der Meer scans in order to determine the luminosity [161]. With the time information provided by both V0 arrays, additional event selection criteria can be enforced to suppress beam-gas interactions or other background sources as described in Section 4.2.

The To detector [158] consists of two Cherenkov counters at $72.7 \mathrm{~cm}$ and $375 \mathrm{~cm}$ from the interaction point covering the forward pseudorapidities of $4.61<\eta<4.92$ (T0A) and $-3.28<$ $\eta<2.97$ (T0C). One of the main purposes of the T0 is to determine the start time $\left(t_{0}\right)$ for the TOF detector and a wake-up signal for the TRD detector with a precision better than 50 ps. This time is also the exact time of the collision and can be used to determine the position of the primary vertex by $\pm 1.5 \mathrm{~cm}$. With this information, the T0 can also provide a minimum bias event trigger with an approximate efficiency of $40-59 \%$ in pp collisions depending on the trigger condition.

\subsubsection{ALICE software framework and reconstruction process}

In the ALICE collaboration a central software framework based on ROOT [162] is used for reconstruction, data processing and analysis. ROOT was developed at CERN so handle the large amounts of data recorded from the various high energy experiments. It is based on $\mathrm{C}++$, but also provides an extension called Рувоот [162] which allows the usage of Python [163] for data analysis. The central analysis framework of ALICE is split into AliRoot [164] and AliPhysics [165] which handle different steps of the analysis procedure. The AliRoot framework provides an interface to the different Monte Carlo generators like Pythia [166,167], Phojet [168], DPMJET [169-171] or EPOS [172]. It furthermore contains the geometry of the ALICE detector to be used with particle transport tools like GEANT 3 [173], GEANT 4 [174] or FLUKA [175] in order to study and correct for detector effects.

These tools allow to trace particles on their path through the detector material, simulate material interactions like energy loss or photon conversions and return hit signals in active detector 
material similar to actual data. These signals, called hits, also emulate most of the detector readout effects like APD signal shapes or silicon detector signal amplitudes in order to provide a datalike detector response.

The full reconstruction chain, used in data and MC simulations, is shown schematically in Figure 3.18 and explained in great detail in Ref. [137]. This chain starts with the combination of detector hit signals into clusters of these signals where they are thought to originate from the same particle depending on time and spacial information. In the case of the SPD, this information is used to reconstruct the collision vertex (primary vertex) by combining straight lines formed from clusters, called tracklets, in both SPD layers which point to a common point. As this procedure is performed for each combination of tracklets, it can also identify pileup vertices from additional collisions which are dis-

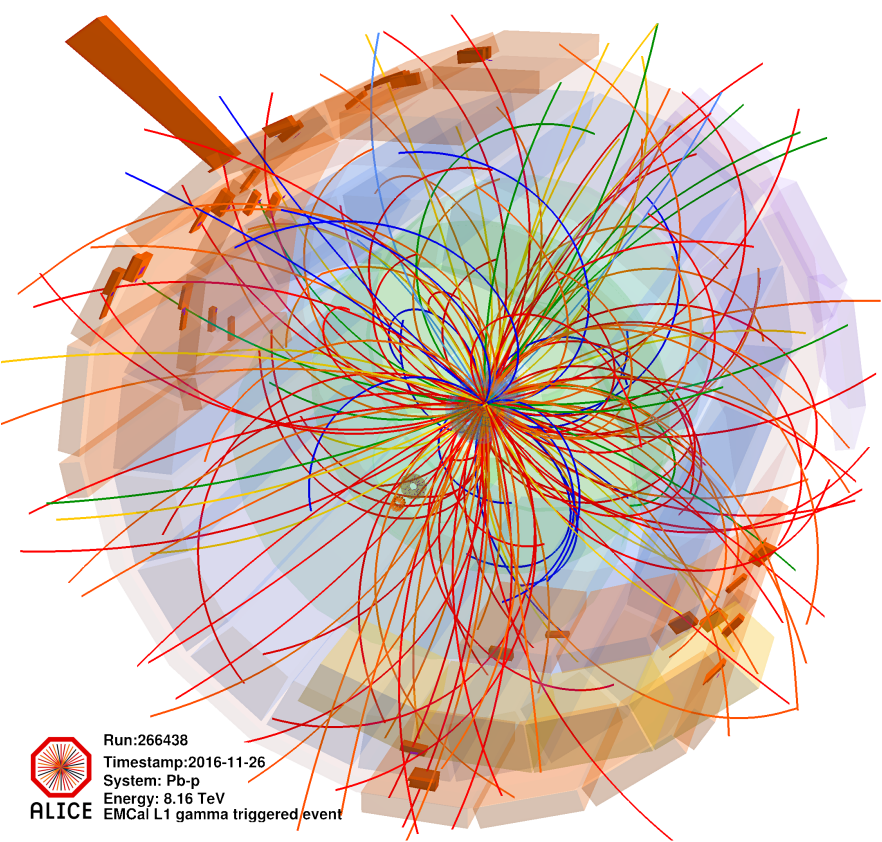

Figure 3.19: View of the ALICE central barrel geometry implemented in AliRoot with the reconstructed tracks and energy deposits in a recorded high multiplicity $\mathrm{p}-\mathrm{Pb}$ collision at $\sqrt{s_{\mathrm{NN}}}=8.16 \mathrm{TeV}$ from 2016 . placed compared to the primary vertex.

The vertex finding probability and its position resolution strongly depend on the number of available tracklets which varies by orders of magnitude going from pp collisions to $\mathrm{Pb}-\mathrm{Pb}$ collision. Therefore, the primary vertex resolution from this procedure is on the order of $150 \mu \mathrm{m}$ for pp collisions and one order of magnitude better in heavy-ion collisions. After the primary vertex finding procedure, the full tracking in ALICE is performed in a three-step process based on an inward-outward scheme. It starts with finding track seeds for a Kalman filter in the outer regions of the TPC by combining two TPC clusters together with the primary vertex position. In a subsequent step, the primary vertex constrain is omitted and additional TPC clusters compatible with the current track parameters are added. A track is accepted if it has at least 20 TPC clusters and more than $50 \%$ of the expected number of clusters for a track reaching the given distance in the TPC. During this procedure, the track is also assigned a mass hypothesis based on the energy loss signal $\mathrm{d} E / \mathrm{d} x$ where at least the charged pion mass is assigned omitting any electron hypothesis. This TPC-based procedure can find tracks with an efficiency of more than $70 \%$ at $p_{\mathrm{T}}=50 \mathrm{MeV}$ and with more than $85 \%$ around $p_{\mathrm{T}} \approx 10 \mathrm{GeV} / c$. Once the inner boundary of the TPC is reached, the tracking algorithm propagates the tracks to the outer ITS layers in order to create a seed for the ITS track finding procedure. Similar to the TPC algorithm, this tracking is also performed with and without the vertex constraint and information from each ITS layer is added to the track if possible. The tracking algorithm penalizes missing information from ITS layers by increasing the $\chi^{2}$ of the track and continues until the innermost layer is reached. Remaining clusters in the ITS after all TPC tracks have been propagated are then used in a second ITS-only track finding approach.

Every track is then propagated outwards starting with the extrapolated point closest to the vertex and using the track parameters to associate clusters from each ITS layer and the TPC. Wrongly assigned or missing clusters are removed or added, respectively, and the track is continued to 
larger radii including the time-of-flight information of the TOF to verify the mass hypothesis as well as tracklets in the TRD to further constrain the tracks.

The last of the three tracking steps is an additional inwards propagation from the outer boundary of the TPC where a refitting with the previously found clusters is performed and the final track parameters are determined. These tracks are used to determine the interaction vertex again but provide an improved position resolution compared to the SPD tracklet approach. Each track is also checked for a kink typology meaning a track that shows a deflection by a small angle at a certain point. Such a topology is possible for charged particles that decay into another charged particle plus a neutral particle. The most prominent cases for this are the $\mathrm{K}^{ \pm} \rightarrow \pi^{ \pm}+\pi^{0}$, $\pi^{ \pm} \rightarrow \mu \nu$ and $\mathrm{K}^{ \pm} \rightarrow \mu \nu$ decays.

A fully reconstructed EMCal-triggered event in $\mathrm{p}-\mathrm{Pb}$ collisions at $\sqrt{s_{\mathrm{NN}}}=8.16 \mathrm{TeV}$ is shown in Figure 3.19 showing reconstructed charged particle tracks as curved lines and energy deposits in the electromagnetic calorimeters as boxes whose size reflects the signal amplitude. The figure furthermore shows the central barrel detector geometry on a simplified level as implemented in the AliRoot framework. In addition to the primary vertex reconstruction and global tracking, also secondary vertices from particles, that either have a long decay length or that interact with the detector material, are reconstructed in ALICE. This includes decays of neutral particles, which show a 'V-shaped' topology, and are referred to as $\mathrm{V}^{0}$ candidates. Examples for such $\mathrm{V}^{0}$ candidates are $\mathrm{K}_{\mathrm{S}}^{0} \rightarrow \pi^{+} \pi^{-}$or $\Lambda^{0} \rightarrow p \pi^{-}$decays or photon conversions $\gamma \rightarrow e^{+} e^{-}$where the decay vertex of the particle can be reconstructed as the distance of closest approach of the oppositely charged tracks. Such decays are visualized in Figure 3.20 exemplary for $\mathrm{K}_{\mathrm{S}}^{0}$ and $\Xi^{-}$, where the latter is a cascaded decay with an intermediate $\Lambda^{0}$ decay. The figure shows the hits in the ITS as well as the reconstructed secondary tracks. A track is classified as secondary, if it shows a distance of closest approach (DCA) of more than $0.5 \mathrm{~mm}$ or $1 \mathrm{~mm}$ for pp and $\mathrm{Pb}-\mathrm{Pb}$ collisions, respectively. In addition, tracks for secondary vertex finding are required to be of opposite charge. The secondary vertex position is determined as the point of closest approach (PCA) of the two tracks, which must be less than $1.5 \mathrm{~cm}$. The resulting momentum vector $\bar{p}_{\text {pair }}$, obtained from the combined momenta of the individual tracks, must point to the primary vertex and fulfill $\cos \theta<0.85$.

In ALICE, two different $\mathrm{V}^{0}$ finding algorithms are employed. The on-the-fly $\mathrm{V}^{0}$ finder applies the Kalman filter [176, 177] during the reconstruction stage, thus it has access to raw cluster information for tracking, which allows for an excellent position and momentum resolution. In addition, the access to the track clusters enables the use of a causality cut, which exploits that no track clusters can be found at smaller radial positions than the actual decay point. The main caveat of this $\mathrm{V}^{0}$ finder is the inability to be rerun on analysis level, for example with looser selection criteria, as the track clusters are only available during the

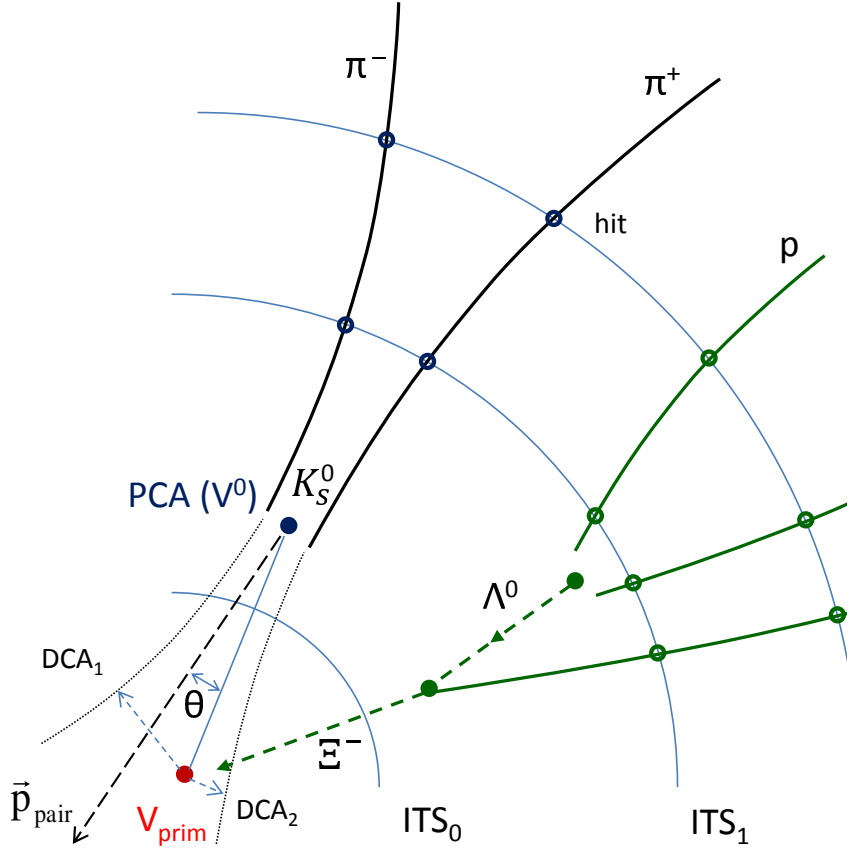

Figure 3.20: Secondary vertex reconstruction sketch for decays of $\mathrm{K}_{\mathrm{S}}^{0}$ and $\Xi^{-}$[137]. Hits in different ITS layers are indicated as circles and reconstructed tracks as solid lines. Dotted lines represent the extrapolated momentum vectors of the $\mathrm{V}^{0}$-particles in direction of the primary vertex. 
initial reconstruction stage. The alternative algorithm is the offline $\mathrm{V}^{0}$-finder, which performs its vertex finding on the already reconstructed tracks. It can therefore be re-run on analysis level, but results in a worse momentum and vertex resolution compared to the on-the-fly $\mathrm{V}^{0}$-finder due to the lack of the causality cut and the full tracking information. In addition, this algorithm allows to change the selection criteria, making it more flexible for different analyses. 


\section{Data sets}

This section introduces all data that is used in the analysis as well as the simulations that are used to correct the data. The event selection criteria are discussed with special focus on pileup removal. In addition, the minimum bias and special event triggers during data taking are explained.

\begin{tabular}{|c|c|c|c|c|c|c|c|c|c|c|}
\hline \multicolumn{3}{|c|}{ LHC RUN 1} & \multicolumn{3}{|c|}{ LS 1} & \multicolumn{3}{|c|}{ LHC RUN 2} & \multicolumn{2}{|c|}{ LS2 } \\
\hline 2010 & 2011 & 2012 & 2013 & 2014 & 2015 & 2016 & 2017 & 2018 & 2019 & 2020 \\
\hline
\end{tabular}

Figure 4.1: Schematic overview of the main LHC physics beam configurations over time until 2020. The approximate duration of each data taking campaign is indicated by the width of the correspondingly colored bar in the lowest row.

\subsection{Data samples}

The LHC has provided ALICE with a multitude of particle beam configurations ranging from symmetrical proton-proton $(\mathrm{pp})$, lead-lead $(\mathrm{Pb}-\mathrm{Pb})$ and xenon-xenon (Xe-Xe) collisions to asymmetric proton-lead $(\mathrm{p}-\mathrm{Pb})$ collisions. The center-of-mass energy in all collision systems has increased over time and by now ALICE has recorded data in pp collisions at six different center-ofmass energies ranging from $\sqrt{s}=0.9 \mathrm{TeV}$ up to $13 \mathrm{TeV}$ as well as p-Pb collisions at $\sqrt{s_{\mathrm{NN}}}=5.02$ and $8.16 \mathrm{TeV}$. The heavy ion program contained $\mathrm{Pb}-\mathrm{Pb}$ collisions at $\sqrt{s_{\mathrm{NN}}}=2.76$ and $5.02 \mathrm{TeV}$ as well as Xe-Xe collisions at $\sqrt{s_{\mathrm{NN}}}=5.44 \mathrm{TeV}$. A timeline with the various beam configurations is shown in Figure 4.1 also highlighting the approximate duration of the different data taking campaigns.

The data used in this thesis is from both, LHC Run 1 and LHC Run 2 and follows a general naming scheme within ALICE where the name of the data set begins with the year in which it was recorded (e.g. 2013), followed by a letter indicating the so-called period of data taking. During one period (which corresponded in LHC Run 1 to roughly one month of data taking) the detector setup, beam configuration as well as event triggers are kept as stable as possible without introducing significant changes (e.g. inverting the beams from $\mathrm{p}-\mathrm{Pb}$ to $\mathrm{Pb}-\mathrm{p}$ in the LHC). A period is then further divided into so-called runs which stand for a time interval between minutes and several hours in which data was continuously taken without interruptions by the LHC or errors in the ALICE detector systems.

The periods analyzed in this thesis are LHC12[a-i] for pp collision data at $\sqrt{s}=8 \mathrm{TeV}$ which were recorded during 2012. The periods LHC16r and LHC16s were taken in autumn of 2016 and contain collision data from $\mathrm{p}-\mathrm{Pb}$ and $\mathrm{Pb}-\mathrm{p}$ at $\sqrt{s_{\mathrm{NN}}}=8.16 \mathrm{TeV}$, respectively. A full overview of all employed ALICE data samples and Monte Carlo simulations is given in Table 14 in the appendix.

\subsection{Event selection}

The events that are accepted for analysis have to fulfill several requirements related to the event properties. In a first step, the events are required to be of a meaningful physics type for which the so called Physics Selection (PS) in the ALICE AliPhysics framework [165] is used. This selection tool allows to reject calibration events or background events, for example by applying cuts on 
the V0A and V0C time information in order to remove events from beam-gas interactions which compared to an actual collision induce early signals in one of the arrays. Furthermore, a selection on the SPD clusters versus tracklets distribution with $N_{\text {cluster }}>4 \times N_{\text {tracklet }}+65$ allows to remove background and pileup events. This cut relies on the proportionality between SPD clusters and their resulting tracklets where a large number of clusters but low number of tracklets points to background events leaving signals in the SPD. Further event selection criteria are applied in multiple steps in order to remove remaining contributions from in-bunch and out-of-bunch crossing pileup. In-bunch pileup is removed if multiple collision vertices with at least 3 contributing tracklets and at least $8 \mathrm{~mm}$ of separation are reconstructed with the SPD in its readout

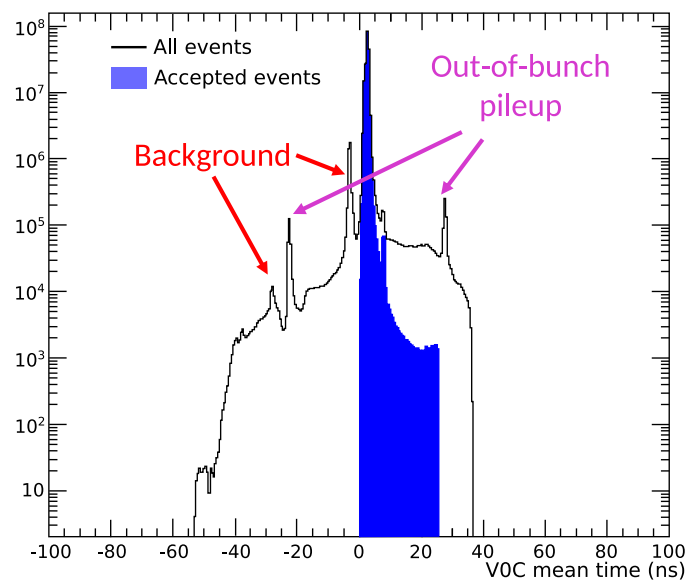

Figure 4.2: Mean V0C time distribution for all events (black) and after background and pileup rejection criteria are applied (blue). window of $300 \mathrm{~ns}$. Due to the large readout window, out-of-bunch pileup from the closest bunch crossing to the triggering event can also be removed with this method. The in-bunch pileup increases with higher luminosities due to the stronger collimation of the beams. In the pp and $\mathrm{p}-\mathrm{Pb}$ datasets the interaction rates in ALICE resulted in $3.5 \%$ of events coming from in-bunch pileup. For further out-of-bunch pileup rejection, the so-called past-future rejection is used which uses the V0 information for the surrounding 10 bunch crossings around the trigger event to check for additional activity in the V0 outside of the SPD integration time window. Figure 4.2 shows the effect of the background and pileup removal criteria on the V0C mean time distribution. The clear signals from beam-gas background and out-of-bunch pileup are largely removed. Events entering this analysis need to have a reconstructed primary vertex within $\left|z_{\mathrm{vtx}}\right|<10 \mathrm{~cm}$ from the nominal center of ALICE. This vertex has to have at least one contributing global track or SPD tracklet pointing to it. Both of these criteria ensure that a large fraction of the created particles traverses the central barrel detectors

in their active areas. Events also have to fulfill the minimum bias trigger condition. In ALICE this is based on signal detection in the SPD and VZERO detectors. The VZERO can additionally separate physics events from beam-gas interactions, for which the time difference between the nominal collision, based on the LHC clock, and the signals in the V0A and V0C arrays is used. For a nominal collision, particles reach the V0A (V0C) detectors about 11.3 (3.0) ns after the LHC-based collision time depending on where in the elongated bunches the collision happens. Beam-gas interactions which originate several centimeters up to meters away from the interaction point will induce signals in the VZERO arrays at significantly different times and thus these events can be rejected. The correlation between the V0A and V0C time is shown in Figure 4.3 where the black box indicates events from actual beam-beam interactions [161]. During the early data taking campaigns

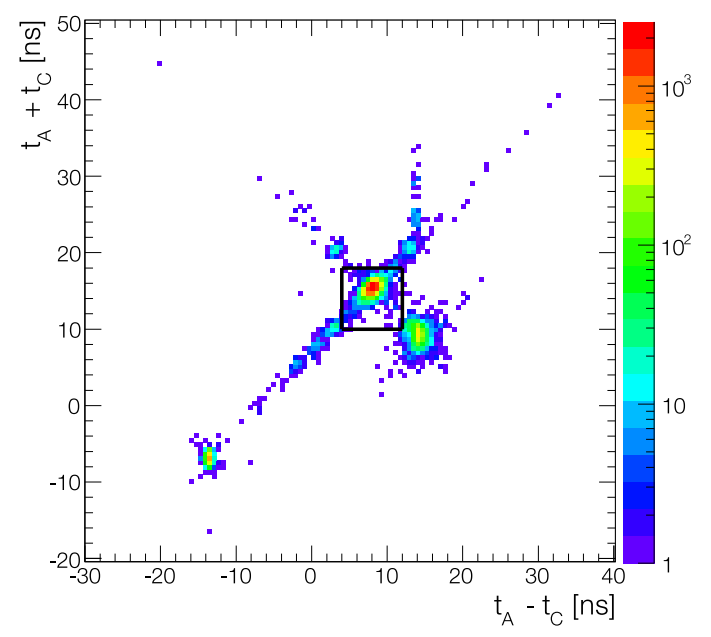

Figure 4.3: Correlation between the VOA and V0C arrival times relative to the bunch crossings. The black box indicates events from beam-beam interactions. [161] 
in 2009-2011, the minimum bias trigger V0OR (also called INT1) required a signal in either the SPD or any of the VZERO detectors. This rather loose requirement was performing sufficiently well in the moderate luminosity environment at the time. With higher luminosities, the minimum bias trigger condition was adapted to better suppress background events and to cope with the higher readout rate. The optimized trigger V0AND (INT7) requires a coincidence of signals in both VZERO detectors (V0A and V0C). Based on these criteria, the measurements presented in this thesis are normalized by a corrected number of events to account for the events that are lost when no vertex can be reconstructed via

$$
N_{\text {norm }, \text { evt }}=N_{\mathrm{Y}, \mathrm{vtx},\left|z_{\mathrm{vtx}}\right|<10 \mathrm{~cm}}+\frac{N_{\mathrm{Y}, \mathrm{vtx},\left|z_{\mathrm{vtx}}\right|<10 \mathrm{~cm}}}{N_{\mathrm{Y}, \mathrm{vtx},\left|z_{\mathrm{vtx}}\right|<10 \mathrm{~cm}}+N_{\mathrm{Y}, \mathrm{vtx},\left|z_{\mathrm{vtx}}\right|>10 \mathrm{~cm}}} N_{\mathrm{Y}, \mathrm{no} \mathrm{vtx}},
$$

where $Y$ stands for the used event trigger (minimum bias or EMCal triggers) as explained in the next section. The variable $N$ denotes the number of events with a reconstructed vertex $\left(N_{\mathrm{vtx}}\right)$, with a vertex within $\pm 10 \mathrm{~cm}$ of the IP in $z$ direction $\left(N_{\left|z_{\mathrm{vtx}}\right|<10 \mathrm{~cm}}\right)$ or with no reconstructed vertex $\left(N_{\text {no vtx }}\right)$. The correction term is negligible in $\mathrm{p}-\mathrm{Pb}$ collisions due to the much higher average multiplicity compared to pp collisions and the resulting vanishing fraction of events without a reconstructable vertex.

The integrated luminosity of each trigger sample is required to convert the measurements from invariant yield to an invariant cross-sections. It is calculated according to

$$
\mathscr{L}_{\text {int }}=\frac{N_{\text {trig,norm }}}{\sigma_{\mathrm{MB}}} \times R F_{\text {trig }},
$$

where $N_{\text {trig,norm }}$ is the number of events recorded with the corresponding event trigger and corrected according to Equation 13. The minimum bias trigger cross-section $\sigma_{\mathrm{MB}}$ was determined from van der Meer scans to be $\sigma_{\mathrm{MB}}^{\mathrm{pp}}=55.8 \pm 1.45 \mathrm{mb}$ for pp [178], $\sigma_{\mathrm{MB}}^{\mathrm{pPb}}=2.09 \pm 0.04 \mathrm{~b}$ for $\mathrm{p}-\mathrm{Pb}$ (LHC16r) and $\sigma_{\mathrm{MB}}^{\mathrm{Pbp}}=2.10 \pm 0.04 \mathrm{~b}$ for $\mathrm{Pb}-\mathrm{p}$ (LHC16s) [179]. Special event triggers in ALICE allow to inspect a much higher luminosity than the minimum bias trigger. Equation 14 therefore also contains the trigger rejection factor $R F_{\text {trig }}$ which denotes this enhancement and is explained in the next section. The integrated luminosities of each different data sample used in the analysis are listed in Table 2 for $\mathrm{pp}$ and $\mathrm{p}-\mathrm{Pb}$. For the $\mathrm{p}-\mathrm{Pb}$ data taking campaign, ALICE employed multiple so-called readout clusters which are a set of detector systems that are used to record a triggered event. Certain detectors, like the SDD, are excluded from the readout if they are found to be busy when the trigger is issued. This allows for significantly higher event recording rates compared to waiting for all detector systems to be ready. An additional special readout cluster without the TPC in the readout was used during the $\mathrm{p}-\mathrm{Pb}$ data taking in order to enhance the event counts for the EMCal triggers. Table 2 therefore lists the integrated luminosities separately for the cases with and without the TPC in the readout. The special readout cluster provides up to a factor three higher statistics by not requiring the TPC in the event record.

\subsubsection{EMCal-triggered data}

Aside from the minimum bias triggered data, this analysis uses EMCal Level-0 and Level-1 triggered data to enhance the statistics at high $p_{\mathrm{T}}$. The hardware implementation and design principle of these triggers are explained in detail in Section 3.2.1.5. For the pp analysis, one L0 (EMC7) and one L1 trigger (EGA) were in use while for $\mathrm{p}-\mathrm{Pb}$ the L0 trigger was only flagging minimum bias events in case they also fulfilled the trigger condition. However, in the $\mathrm{p}-\mathrm{Pb}$ data, two L1 triggers (EG2 and EG1) are available with thresholds as listed in Table 2. In order to 

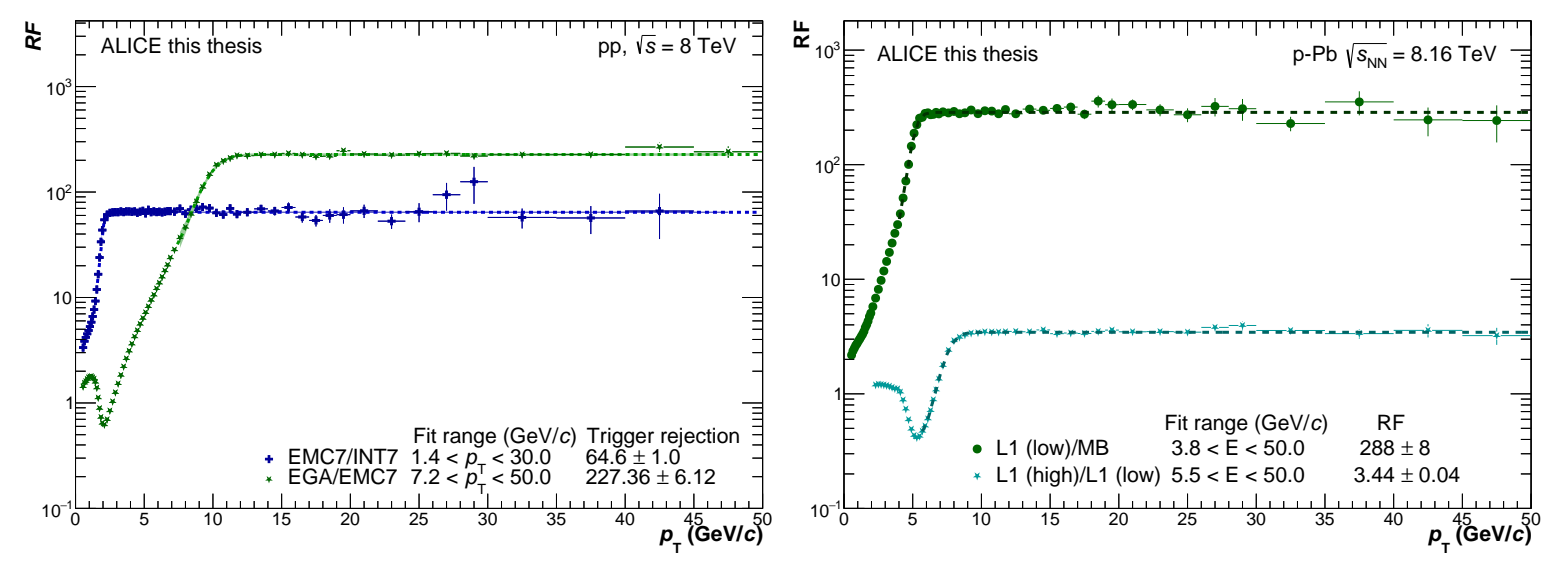

Figure 4.4: Trigger rejection factors in pp (left) and $\mathrm{p}-\mathrm{Pb}$ (right) for the EMCal L0 and L1 triggers with respect to the next lower threshold trigger. The plateau regions are fitted with error functions (Equation 15) in the ranges as written in the legends and indicated by the dotted lines including a systematic uncertainty band from variations of the fitting range.

prevent double counting of triggered events where multiple trigger conditions are fulfilled, each event is only associated with the lowest trigger that fired. This is especially important for the EMCal L1 triggers which had their readout rate reduced by trigger-dependent factors during data taking.

When using the triggered data for analysis, the enhancement factor of each trigger needs to be determined in order for the measurements to be scaled correctly to the minimum bias baseline. The so-called trigger rejection factor, $R F_{\text {trigg }}$, is used for this purpose. It is defined in this analysis as the ratio of the cluster energy spectra from the triggered data sample to that of the minimum bias sample. Under the assumption that the trigger does not modify the reconstruction efficiency of the clusters, this ratio should become constant at high energies. The trigger rejection factors are obtained from error function [180] fits on the cluster spectra ratios according to

$$
R F\left(p_{\mathrm{T}}\right)=R_{0}+\tau \cdot \operatorname{erf}\left(\frac{p_{\mathrm{T}}-p_{\mathrm{T}, 0}}{\sqrt{2} \cdot a}\right),
$$

with the global offset $R_{0}$, a scaling variable $\tau$, free parameters $p_{\mathrm{T}, 0}$ and $a$, as well as the error function $\operatorname{erf}$, which is defined as

$$
\operatorname{erf}(x)=\frac{2}{\sqrt{\pi}} \int_{x}^{\infty} e^{-t^{2}} \mathrm{~d} t .
$$

From this, $R F$ can be determined via $R F=R_{0}+\tau$ with the associated uncertainty being the Gaussian error propagation of the $R_{0}$ and $\tau$ fit uncertainties. Using the error function, instead of just a constant fit in the plateau region, allows to constrain $R F$ with the turn-on part of the ratio, thus significantly reducing uncertainties. Due to the limited statistics of the low threshold and minimum bias trigger samples, the rejection factor is determined in steps where the cluster spectrum ratio is always determined with respect to the next lower trigger. This prevents unnecessary large uncertainties in the ratios which would in every case be dominated by the minimum bias spectrum. The trigger rejection factors for the pp triggers (EMC7/INT7 and EGA/EMC7) as well as for the p-Pb triggers (EG2/INT7 and EG1/EG2) are shown in Figure 4.4 together with the error function fits and their corresponding uncertainty bands. The uncertainty is determined via symmetric variations of the fitting range around the default low $E$ starting value and is considered as systematic uncertainty on the integrated luminosity. Aside from the plateau region, the trigger rejection factor shows a distinct turn-on at lower energies which agrees with the trigger threshold as listed in Table 2 and can be described by the error 


\begin{tabular}{lllccc}
\hline \multirow{2}{*}{ System } & $\begin{array}{l}\text { Trigger } \\
\text { Name }\end{array}$ & $\begin{array}{l}\text { Threshold } \\
(\mathrm{GeV})\end{array}$ & $\begin{array}{c}\text { Trigger rejection } \\
\text { factor } R F\end{array}$ & \multicolumn{2}{c}{$\mathscr{L}_{\text {int }}\left(\mathrm{nb}^{-1}\right)$} \\
& $\mathrm{w} / \mathrm{TPC}$ & $\mathrm{w} / \mathrm{o}$ TPC \\
\hline $\mathrm{pp}$ & INT7 & 0 & 1 & 1.94 & - \\
& EMC7 & 2.0 & $64.6 \pm 1.0$ & $40.9 \pm 1.1$ & - \\
& EGA & 10 & $(14.7 \pm 0.6) \times 10^{3}$ & $615 \pm 15$ & - \\
\hline $\mathrm{p}-\mathrm{Pb}$ & $\mathrm{INT7}$ & 0 & 1 & 0.018 & 0.041 \\
& EG2 & 5.5 & $288 \pm 8$ & $0.081 \pm 0.002$ & $0.206 \pm 0.005$ \\
& EG1 & 8.0 & $991 \pm 29$ & $1.42 \pm 0.04$ & $5.67 \pm 0.16$ \\
\hline
\end{tabular}

Table 2: Integrated luminosity for the different EMCal triggers used in $\mathrm{pp}$ and $\mathrm{p}-\mathrm{Pb}$ together with their approximate trigger threshold during data taking and the trigger rejection factor relative to the minimum bias trigger. For $\mathrm{p}-\mathrm{Pb}$ the values are given for data with $\mathrm{TPC}$ in readout (for PCM and PCM-EMC) and without TPC in readout (for EMC and MEMC). The uncertainty from the determination of the $\mathrm{MB}$ trigger cross section of $1.9 \%$ for $\mathrm{p}-\mathrm{Pb}$ and $2.6 \%$ for $\mathrm{pp}$ is not included.

function. The width of this turn-on stems from the implementation of the trigger as an analog signal threshold in the detector hardware. When these analog signals are converted to energy values, they can differ between trigger channels depending on their calibration and thus result in a smeared trigger threshold energy. The table also lists the final trigger rejection factors with respect to the minimum bias trigger as well as the resulting integrated luminosity based on Equation 14.

\subsection{Monte Carlo simulations}

Full event and detector simulations are needed in order to determine correction factors like the kinematic acceptance, the reconstruction efficiency or the purity. PYTHIA8 [166, 167], PHOJET [168], DPMJET-III [169] and EPOS LHC [172] Monte Carlo event generators with subsequent detector simulation using GEANT 3 [173] are used to provide the full event and detector response information for data correction. In the following, an overview of the various event generators as well as the detector response via GEANT 3 is provided.

\section{Pythia}

The general purpose event generator PYTHIA8 $[166,167]$ is the most widespread generator used for comparisons to LHC physics capable of generating proton, lepton and nuclei collisions. It serves not only for generator level studies but also as input for full detector simulations that are essential for most physics measurements. The PYTHIA generator uses only the leading order (LO) of the strong interaction parameter $\alpha_{s}$ expansion to describe hard $2 \rightarrow 2$ and $2 \rightarrow 1$ QCD scattering processes (e.g. $g g \rightarrow g g$ and $g q \rightarrow g q$ ) which are the dominant source for jet and hadron production. Matrix elements are used as implementation for the transition from initial to final state effects. Following the hard processes, partonic interactions are described by a parton shower including Initial State Radiation (ISR) and Final State Radiation (FSR). PyTHIA also includes the simulation of multi parton interactions (MPIs) where more than one partonic interaction can occur in an event. At LHC energies, events contain around 4-10 interactions [181]. Furthermore, the implementation of Color Reconnection (CR) [182] assigns partons a color and a corresponding anti-color partner to form a color dipole which is connected via strings. In this model, gluons which carry both color and anti-color correspond to kinks in the strings. CR then allows the strings to be switched between partons and as a result decrease the overall string length. Hadronization in PYTHIA is simulated according to the Lund String fragmentation 
model [183] while particle decays are handled via branching ratios and decay properties provided by the particle data group [184]. PYTHIA moreover allows to enhance jets in the generated sample by enforcing a selection on the hard scattering parton momentum $p_{\mathrm{T}}^{\text {hard }}$. Such a selection provides larger statistics at high particle momentum without the need to simulate massive amounts of CPU and storage intensive events. Monte Carlo production where a $p_{\mathrm{T}}^{\text {hard }}$ selection is enforced are called Jet-Jet MC simulations and the Jet-Jet productions used in the presented analyses are listed in Table 14. As the selection of events for a given $p_{\mathrm{T}}^{\text {hard }}$ requires several simulations of initial conditions $\left(N_{\text {trials }}\right)$, the resulting spectrum must be weighted based on the average cross section of the hard scattering $\sigma_{\text {evt }}$ determined by PYTHIA. The weight for all events in a given $p_{\mathrm{T}}^{\text {hard }}$ slice is given by

$$
\omega_{\mathrm{JJ}}=\frac{\sigma_{\text {evt }}}{N_{\text {trials }} / N_{\text {gen.evt. }}},
$$

which includes the total number of events generated in the $p_{\mathrm{T}}^{\text {hard }}$ slice $\left(N_{\text {gen.evt. }}\right)$. In the presented analyses, further requirements are imposed on the PYTHIA Jet-Jet MC simulations on the transverse momentum of single particles. No particle in the event may have a $p_{\mathrm{T}}$ larger than $1.1 \cdot p_{\mathrm{T}}^{\text {hard }}$ of the event and in addition no jet in the event may exceed $p_{\mathrm{T} \text {,jet }}>2.5 p_{\mathrm{T}}^{\text {hard }}$. These criteria ensure that unwanted behaviors of the generator are excluded from the analysis which would show up as outliers in the particle $p_{\mathrm{T}}$ spectra.

\section{Phojet}

The Monte Carlo event generator Phojet [168] is able to describe hadron-hadron, photonhadron and photon-photon interactions at high energies [185] due to the comination of soft hadronic processes with the Dual Parton Model (DPM) [186]. It allows to calculate multiple soft and hard processes [187] where the multiplicities are calculated using a unitarization scheme [188].

\section{DPMJET}

The DPMJET-III [169] multipurpose event generator resulted from the combination of the Dpmjet-II $[170,171]$ and DTUnUC-2 [189] event generators into a unified framework. Due to the individual components, the generator is based largely on Phojet with the inclusion of the Dual Parton Model [186] in order to be able to describe low $p_{\mathrm{T}}$ particle production via soft multiparticle production. Nuclear cross sections in this generator are calculated via the Glauber-Gribov formalism as implemented in the code of the Glauber Diagram Generator (Diagen) [190].

\section{EPOS LHC}

The EPOS Monte Carlo event generator can simulate minimum bias or centrality dependent collisions of heavy ions. The model of the updated EPOS LHC [172] version is tuned to LHC data up to $\sqrt{s}=8 \mathrm{TeV}$ while the latest $\sqrt{s}=13 \mathrm{TeV}$ data is not yet tuned for. In this version flow is also parameterized differently in pp collisions compared to the large volume producing heavy-ion collisions. The model itself is based on the Gribov Regge multiple scattering framework [191] and uses the color exchange mechanisms of string excitation. For the simulation of the reaction volume this model uses a division into the core and the corona region with independent evolution of each volume using hydrodynamics and string decays, respectively.

\section{GEANT3}

The propagation of generated particles through the detector material as well as their interactions in the material are described by the GEANT3 package. GEANT provides particle hits in the material and takes into account the implemented detector configuration and 
its detailed material properties for the simulation. Effects like energy loss, photon conversions, nuclear interactions or scattering processes are all simulated following their basic theoretical models.

\subsubsection{EMCal trigger simulation}

The Monte Carlo simulations used for the correction of the data are by default not simulating the event selection of the EMCal L0 and L1 triggers. As described in Section 4.3 the simulations with a high $p_{\mathrm{T}}$ reach are based on a $p_{T}^{\text {hard }}$ dependent event selection with possible additional event selection on decay photons pointing to the EMCal surface above a certain energy threshold. For the invariant mass based analyses these criteria are not sufficient in order to describe the correction factors for clusters close to the trigger turn-on. An additional trigger simulation, called the trigger maker, is therefore applied for Monte Carlo simulations in order to mimic the Level-0 and Level-1 EMCal trigger event selection as described in Section 3.2.1.5. For the trigger emulation, the trigger patches from FastOR and FEE data are inspected and checked to be above the thresholds which were present dur-

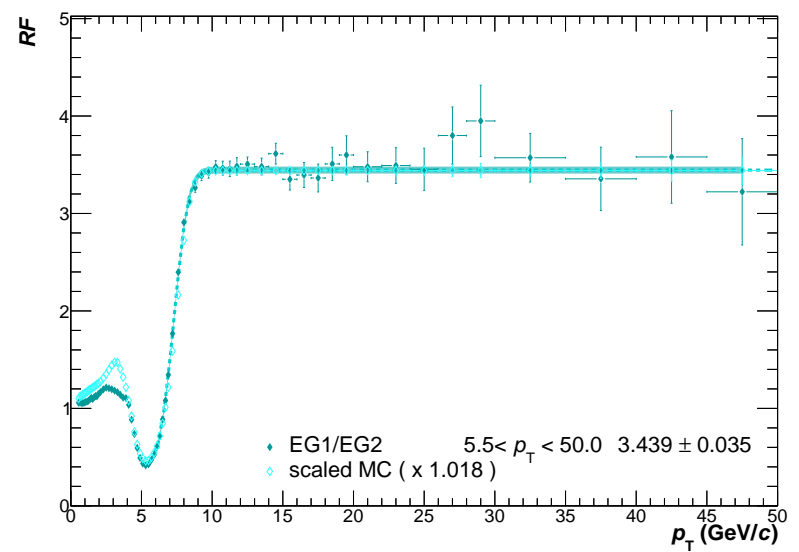

Figure 4.5: Comparison between the EMCal L1 (high) trigger rejection factor in data with the ratio of the trigger emulated cluster spectra in simulation. The Monte Carlo ratio is scaled to data in the plateau region to allow for a shape comparison. ing data taking. The trigger-dependent patch sizes as well as sliding windows are used for the trigger decision. As shown in Figure 4.5 the trigger maker is able to describe the trigger turn-on position and its width in near perfect agreement with data. This ensures an optimal description of the reconstruction efficiency down to energies close to the trigger turn-on in the presented analyses. 


\section{Photon Reconstruction}

The common observable for all analyses presented in this thesis are photons. They are needed for the reconstruction of light neutral mesons in their two-photon decay channel as well as for inclusive and direct photon measurements. This chapter therefore explains the two main methods to measure photons, with detailed descriptions of the necessary selection criteria, as well as a breakdown of the method-dependent calibration procedures. Several of the calibration procedures are in the process of being published as part of the EMCal performance paper [150].

\subsection{Photon Conversion Method (PCM)}

Photons traversing the detector material can convert into electron-positron pairs depending on the available detector material as seen in Figure 5.1 [192]. In ALICE, photons traverse approximately $0.1 X / X_{0}$ radiation lengths of material from the interaction point up to a radius of $180 \mathrm{~cm}$. Using the central barrel tracking detectors ITS and TPC, we can reconstruct the resulting oppositely charged tracks and pass them to a secondary vertex finder in order to obtain the $\mathrm{V}^{0}$ candidates. The secondary vertex finders in ALICE have been explained in Section 3.2.2 and for this analysis the higher efficiency on-the-fly $\mathrm{V}^{0}$-finder is utilized.

The $\mathrm{V}^{0}$ candidate resolution is further optimized for photons by applying the restriction that the decay particles must be parallel to each other at their point of creation. This can be required as the massless

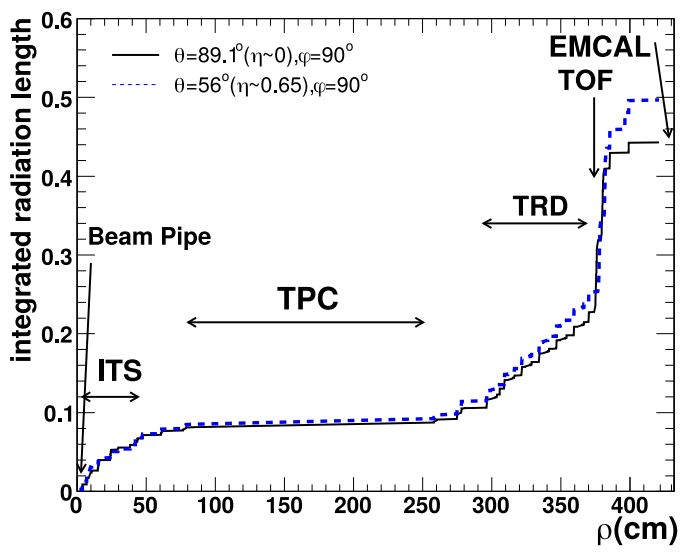

Figure 5.1: Material budget in terms of integrated radiation length in the ALICE central barrel for radii up to the EMCal. Adapted from [192]. photons convert into electron-positron pairs with zero opening angle. This optimization is described in detail in Refs. [193,194]. Distinct selection criteria are applied on the tracks of the $\mathrm{V}^{0}$ candidates in order to obtain a high purity and a high efficiency for the photon reconstruction. A full overview of all selection criteria is given in Table 3 for the track selection and Table 4 for the photon candidate selection. In the following, the individual criteria are explained in detail first for the track selection and then for the photon candidates.

The $\mathrm{V}^{0}$ candidate tracks are required to have a minimum transverse momentum of $0.05 \mathrm{GeV} / c$ in order to allow for the track to reach the TPC. Furthermore, the tracks are required to be within the geometrical acceptance of the ITS and the TPC and thus within a pseudorapidity window

\begin{tabular}{lll}
\hline Track cuts & & \\
\hline Quality & min. track $p_{\mathrm{T}}$ & $p_{\text {T,track }}>50 \mathrm{MeV} / c$ \\
& TPC clusters & $\frac{N_{\text {cluster TPC }}}{N_{\text {findable clusters }}}>60 \%$ \\
\hline \multirow{2}{*}{ Acceptance } & pseudorapidity & $|\eta|<0.9$ \\
& azimuth & $0<\varphi<2 \pi$ \\
\hline \multirow{2}{*}{ TPC PID } & $\mathrm{e}^{ \pm}$selection & $-3 \sigma<\mathrm{n} \sigma_{e}<4 \sigma$ \\
& $\pi^{ \pm}$rejection & $0.4<p_{\mathrm{T}}<3.5 \mathrm{GeV} / c: \mathrm{n} \sigma_{\pi}<1 \sigma$ \\
& & $p_{\mathrm{T}} \geq 3.5 \mathrm{GeV} / c: \mathrm{n} \sigma_{\pi}<0.5 \sigma$ \\
\hline
\end{tabular}

Table 3: Track selection cuts used for the PCM and PCM-EMC analyses. 

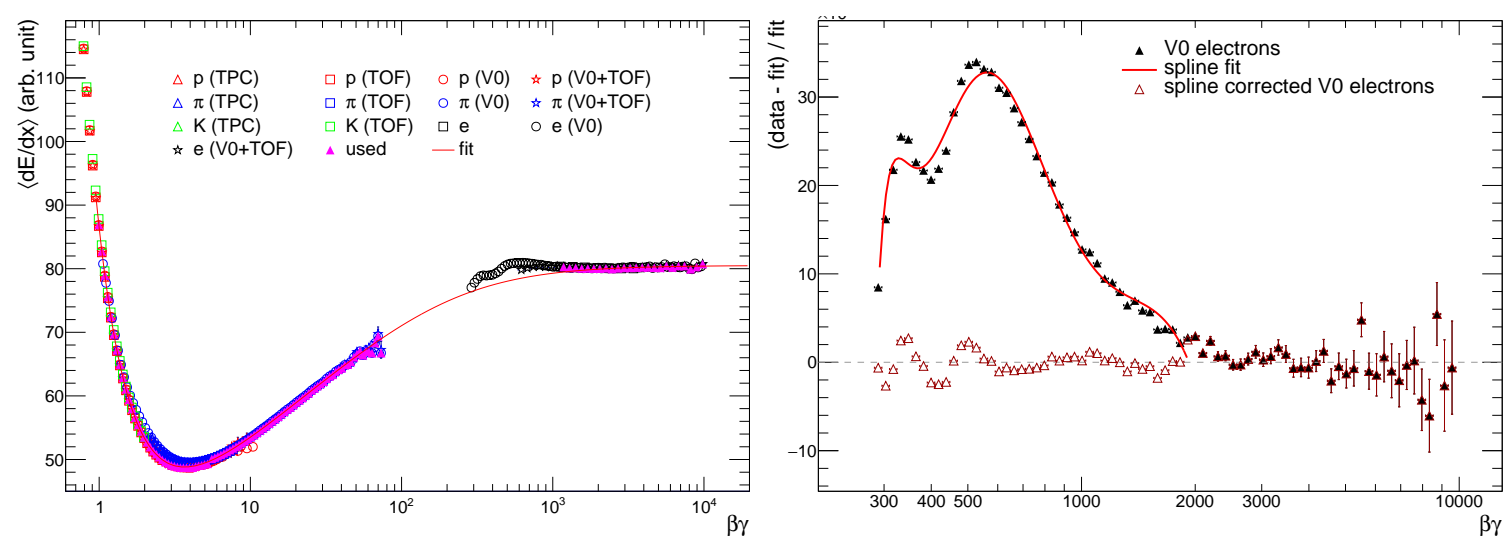

Figure 5.2: Left: Mean energy loss signal for four particle species identified using various detector systems versus $\beta \gamma$ together with a Bethe Bloch fit function shown in red. Right: Comparison of the electron data to the Bethe Bloch fit with the spline parametrization of the residual difference. The spline-corrected data is shown in open markers.

of $|\eta|<0.9$ with no restriction in azimuth. A further quality cut on the reconstructed tracks based on the number of reconstructed TPC clusters of more than $60 \%$ of the maximum possible TPC clusters for the given track is applied. This percentage-based criterium instead of a fixed minimum number of clusters is necessary as the radial position of the conversion as well as the momentum dependent track curvature affect the maximum amount of track points that can be found.

As we are only interested in electrons and positrons for the conversion reconstruction, a particle identification selection on the tracks that are used for $\mathrm{V}^{0}$ reconstruction is performed. For this, the TPC $\mathrm{d} E / \mathrm{d} x$ energy loss signal is used with a cut around the expected energy loss in terms of standard deviations defined as

$$
n \sigma^{\text {part }}=\frac{\langle\mathrm{d} E / \mathrm{d} x\rangle^{\text {meas }}-\langle\mathrm{d} E / \mathrm{d} x\rangle^{\text {spline,part }}}{\sigma(\langle\mathrm{d} E / \mathrm{d} x\rangle)},
$$

where $\langle\mathrm{d} E / \mathrm{d} x\rangle^{\text {meas }}$ is the measured energy loss signal for the track in the TPC, $\langle\mathrm{d} E / \mathrm{d} x\rangle^{\text {spline,part }}$ the expected energy loss signal based on the Bethe Bloch parametrization used for TPC calibration and $\sigma(\langle\mathrm{d} E / \mathrm{d} x\rangle)$ the resolution or width of the Bethe Bloch parametrization.

The Bethe Bloch function for the expected $\mathrm{d} E / \mathrm{d} x$ signal is obtained in a central calibration step for each ALICE data set. The function according to Equation 12 is fitted to data from various
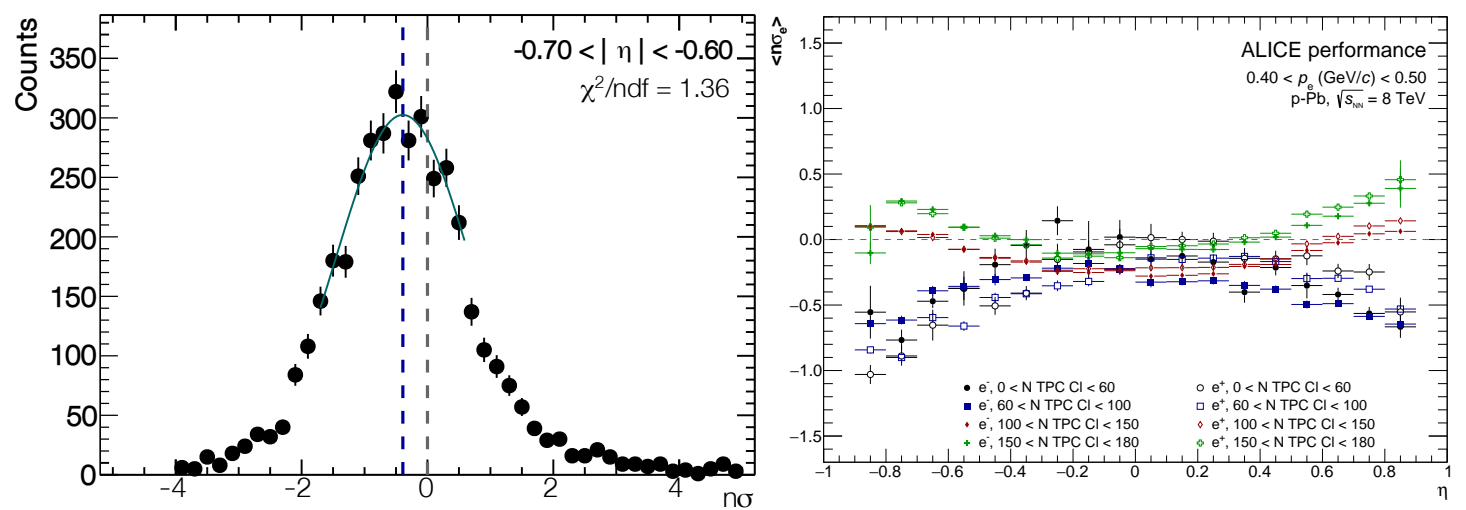

Figure 5.3: Left: $\mathrm{d} E / \mathrm{d} x$ signal projections for the $p_{\mathrm{T}}$ bin $0.1-0.2 \mathrm{GeV} / c$ and $60-100 \mathrm{TPC}$ clusters in multiple pseudorapidity slices. The Gaussian fit for the recalibration (blue) as well as its mean value (dotted line) are shown in addition. Right: Mean $\mathrm{d} E / \mathrm{d} x$ value for each TPC cluster class (separate figures) and for each transverse momentum bin versus $\eta$. 
particle species (protons, charged pions, kaons and electrons) which are identified with TPC, TOF or from $\mathrm{V}^{0}$ candidates as shown in Figure 5.2 (left). The figure shows the mean energy loss signals for these identified particles versus $\beta \gamma$ where the points that are considered for the Bethe Bloch fit are shown in pink and the fit itself in red. As can be seen, the data is in general well described by this fit. However, each particle species slightly deviates from the general Bethe Bloch parametrization for lower values of $\beta \gamma$ where an additional recalibration step is added. This is shown in Figure 5.2 (right) exemplary for electrons where the deviation of the electron data from the fit is visible. The residual difference is parametrized using a spline fit (shown in red) which is then used to shift the data resulting in a per-mille level agreement with the initial Bethe Bloch fit. This procedure is repeated for all particle species entering the initial fit and afterwards a two-dimensional map in $\eta-p_{\mathrm{T}}^{-1}$ is created with the difference between data and expected signal. This map in combination with the Bethe Bloch and spline fits are used to obtain the calibrated energy loss signal on analysis level. For a further improvement of the $\mathrm{d} E / \mathrm{d} x$ selection performance, an additional recalibration of the average TPC energy loss signal to a mean value of 0 depending on $p_{\mathrm{T}}, \eta$ and the mean number of TPC clusters is performed. This is achieved by Gaussian fits to the $\mathrm{n} \sigma \mathrm{d} E / \mathrm{d} x$ signal projections for multiple $p_{\mathrm{T}}$ bins as well as slices of 0.1 in $\eta$ in each $p_{\mathrm{T}}$ bin. An example of these projections is given in Figure 5.3 (left) where a deviation of the mean value from 0 is visible. These gaussian fits are repeated in all $p_{\mathrm{T}}$ and $\eta$ slices for each TPC cluster class in order to obtain an additional recalibration factor that can be applied on analysis level depending on $p_{\mathrm{T}}, \eta$ and the TPC clusters. The resulting calibration factors for an example $p_{\mathrm{T}}$ bin and all TPC cluster slices versus $\eta$ are shown in Figure 5.3 (right). This procedure itself is necessary as the central ALICE calibration does
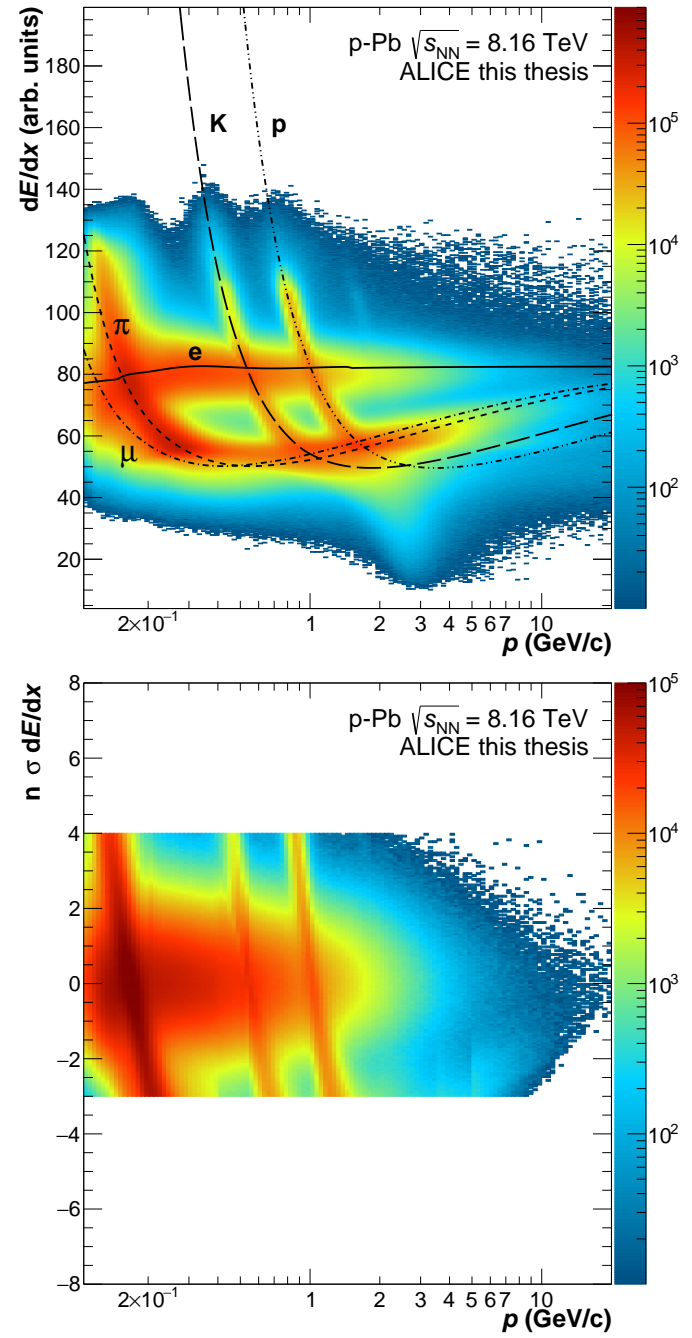

Figure 5.4: TPC energy loss signal $\mathrm{d} E / \mathrm{d} x$ versus track momentum for secondary tracks before cuts (top), except for the cuts imposed by the data format used in the analysis. The distribution after PID cuts in terms of standard deviations from the energy loss hypothesis $n \sigma \mathrm{d} E / \mathrm{d} x$ are applied on data (bottom) is given versus secondary track momentum for $\mathrm{p}-\mathrm{Pb}$ collisions at $\sqrt{s_{\mathrm{NN}}}=8.16 \mathrm{TeV}$. not cover the dependence on the number of available TPC clusters which directly affect the energy loss signal performance. Following this, a selection of $-3 \sigma<n \sigma^{e^{ \pm}}<4 \sigma$ is applied in order to select mainly electron tracks from the sample. However, at low and high track momentum, the energy loss signal of charged pions crosses that of electrons and thus an additional rejection based on the charged pion energy loss hypothesis is applied. This rejection removes all low momentum $\left(0.4<p_{\mathrm{T}}<3.5 \mathrm{GeV} / c\right)$ tracks with a $\mathrm{d} E / \mathrm{d} x$ up to $1 \sigma$ above the expected value for charged pions. The energy loss signal distribution of the TPC is shown in Figure 5.4 once for all tracks (left) and after application of the electron selection and pion rejection cuts (middle and right) for data and simulation. The left plots shows the 


\begin{tabular}{lll}
\hline $\mathbf{V}^{0}$ (photon) cuts & & \\
\hline Acceptance & pseudorapidity & $|\eta|<0.8$ \\
& azimuth & $0<\varphi<2 \pi$ \\
& radius & $5 \mathrm{~cm}<R_{\text {conv }}<180 \mathrm{~cm}$ \\
& $z$-position & $\left|Z_{\text {conv }}\right|<240 \mathrm{~cm}$ \\
\hline Quality & 2D cut on $\left|\psi_{\text {pair }}\right|$ and $\chi_{\gamma}^{2} /$ ndf & $\left|\psi_{\text {pair }}\right|<0.18 \cdot \exp \left(-0.065 \cdot \chi_{\gamma}^{2}\right)$ \\
& cut on $q_{\mathrm{T}}$ & $q_{\mathrm{T}}<0.125 \cdot p_{\mathrm{T}}^{\gamma}$ and $q_{\mathrm{T}}<0.05$ \\
$\quad \& \quad\left|\alpha_{\gamma}\right|$ & $<0.95$ \\
& $\cos \left(\theta_{\text {point }}\right)$ & $>0.85$ \\
\hline
\end{tabular}

Table 4: Photon candidate selection cuts used for the PCM and PCM-EMC analyses.

distribution in arbitrary energy loss units while the other plots are given in terms of $\mathrm{n} \sigma$ around the electron line. Clear lines for various particles species $\left(\mathrm{K}^{ \pm}, \mathrm{p}(\overline{\mathrm{p}}), \mathrm{d}, \mathrm{e}^{ \pm}, \pi^{ \pm}\right)$are visible in these distributions which are strongly suppressed once the PID cuts are applied. Further particle identification criteria could be applied at this point on the energy loss signal provided by the ITS or the timing information of the TOF. While such selections would improve the purity of the $\mathrm{V}^{0}$ even further, they disproportionately decrease the reconstruction efficiency at the same time. It was therefore decided to only rely on the TPC particle identification which results in a purity of about $80 \%$ of the sample.

Further constrains are imposed on the $\mathrm{V}^{0}$ candidates after the track quality has been ensured. The selection criteria from Table 4 are applied which add additional acceptance constrains on the conversion radius of the $\mathrm{V}^{0}$ candidates of $5<R_{\text {conv }}<180 \mathrm{~cm}$ in order to suppress contributions from Dalitz decays of both the $\pi^{0}$ and $\eta$ mesons. In addition, the pseudorapidity of the candidates was restricted to $\left|\eta_{\text {cut }}\right|<0.8$ in order to suppress edge effects in the tracking quality as well as Monte Carlo efficiency discrepancies which arise for larger pseudorapidities. As the pseudorapidity is calculated simply based on the $\mathrm{V}^{0}$ momentum vector in relation to the beam axis, an additional geometrical criterium needs to be fulfilled in order to account for a displacement in $z$ direction of the $\mathrm{V}^{0}$ candidate which could lead to it being partially outside of the detector acceptance. The restriction with $R_{\text {conv }}>\left|Z_{\text {conv }}\right| \cdot S_{\mathrm{ZR}}-Z_{0}$, where $S_{\mathrm{ZR}}=\tan \left(2 \arctan \left(\exp \left(-\eta_{\text {cut }}\right)\right)\right)$ and $Z_{0}=7 \mathrm{~cm}$ is the so-called line-cut which takes care of the geometrical acceptance of the detector when applying the pseudorapidity cut. For the calculation of the line-cut, the secondary vertex coordinates $(Z$ and $R$ ) are determined with respect to the nominal center of the detector $(X, Y, Z)=(0,0,0)$ and the variable shift of the primary vertex in $Z$-direction is accounted for by $Z_{0}$.

As the presented analyses utilize very large data samples with additional focus on high $p_{\mathrm{T}}$ measurements, large efforts have been undertaken in order to increase the efficiency of the PCM method at high transverse momentum. For this, the purity-optimized cuts from these recent measurements $[195,196]$ were further improved by introducing a $p_{\mathrm{T}}$-dependence or by using an improved cut shape to reduce combinatorial background while largely maintaining the same high purity. The first cut that was improved is the two-dimensional selection criterium on the reduced $\chi^{2}$ of the Kalman filter-based photon conversion fit versus $\left|\Psi_{\text {pair }}\right| . \Psi_{\text {pair }}$ is the angle between the $\mathrm{e}^{+} \mathrm{e}^{-}$pair plane and the transverse plane and for its determination tracks are first propagated to a radial distance of $50 \mathrm{~cm}$ from the conversion point. The calculation then follows $\Psi_{\text {pair }}=\arcsin \left(\frac{\Delta \theta}{\xi_{\text {pair }}}\right)$, where $\Delta \theta=\theta_{e^{-}}-\theta_{e^{+}}$stand for the angle in the polar direction and $\xi_{\text {pair }}$ is given by $\xi_{\text {pair }}=\arccos \left(\frac{p_{e^{-} \cdot} \cdot \overrightarrow{e^{+}}}{\left|p_{e^{-}}\right| \cdot\left|p_{e^{+}}\right|}\right)$. 
While for the $\mathrm{e}^{+} \mathrm{e}^{-}$pair $\Delta \theta$ remains constant, their opening angle in the transverse plane increases as an effect of the magnetic field. The $\Psi_{\text {Pair }}$ cut therefore allows to suppress remaining combinations of tracks by limiting the ratio of the relative $\mathrm{e}^{+} \mathrm{e}^{-}$pair opening angle directly after creation to the opening angle when propagated to a radial distance of $50 \mathrm{~cm}$ from the conversion point [197].

True photon candidates are therefore centered around values of 0 with a small, but increasing spread for smaller values of $\chi^{2}$ as shown in Figure 5.5 while combinatorial background is distributed uniformly over the full range. The cut in this distribution has been improved compared to the previously used triangular cut (also shown in the figure) by introducing an exponential function which allows cutting closer to the actual signal and thus removing a significant additional portion of combinatorial background. The improved cut is motivated by the near perfect reproduction of the distribution in simulation and the narrow distribution of true signal which is much better captured with the exponential cut.

From Figure 5.6 a strong background in the $\mathrm{V}^{0}$ sample from $\mathrm{K}_{S}^{0}, \Lambda$ and $\bar{\Lambda}$ is visible in the Armenteros-Podolanski distribution [198]. The plot shows the momentum asymmetry of the daughter particles $\alpha=\left(p_{\mathrm{L}}^{+}-p_{\mathrm{L}}^{-}\right) /\left(p_{\mathrm{L}}^{+}+p_{\mathrm{L}}^{-}\right)$ versus $q_{\mathrm{T}}$ which is the projection of the daughter particle (e.g. $\mathrm{e}^{+}$or $\mathrm{e}^{-}$for conversions) transverse momentum on the $\mathrm{V}^{0}$ candidate momentum given by

$$
q_{\mathrm{T}}=\left|\overrightarrow{p_{e^{-}}}\right| \cdot \sqrt{1-\left(\frac{\overrightarrow{p_{e^{-}}} \cdot \overrightarrow{p_{\gamma}}}{\left|\overrightarrow{p_{e^{-}}}\right| \cdot\left|\overrightarrow{p_{\gamma}}\right|}\right)^{2}} .
$$
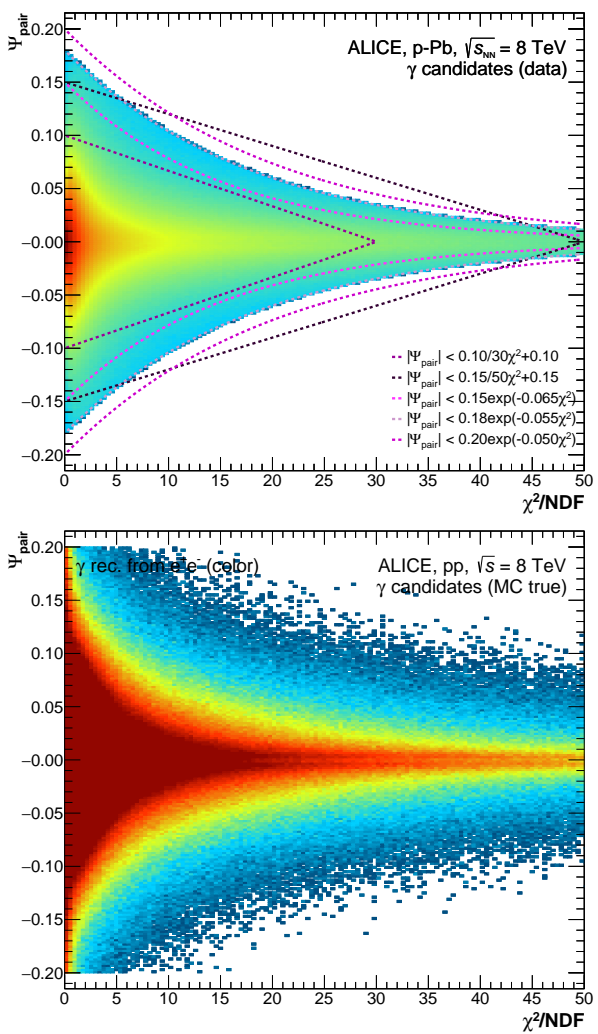

Figure 5.5: Top: Two dimensional distribution of $\chi^{2}$ versus $\left|\Psi_{\text {pair }}\right|$ for $\mathrm{p}-\mathrm{Pb}$ data at $\sqrt{s_{\mathrm{NN}}}=8.16 \mathrm{TeV}$. Bottom: Same distribution for MC simulations for true photons only. Systematic cut variations are indicated by dotted lines.

For true photon conversions small values of $q_{\mathrm{T}}$ are expected as the $\mathrm{e}^{+} \mathrm{e}^{-}$originate from a massless photon and thus they have small (zero) opening angles and their momentum vectors point in the same direction as the mother particle.
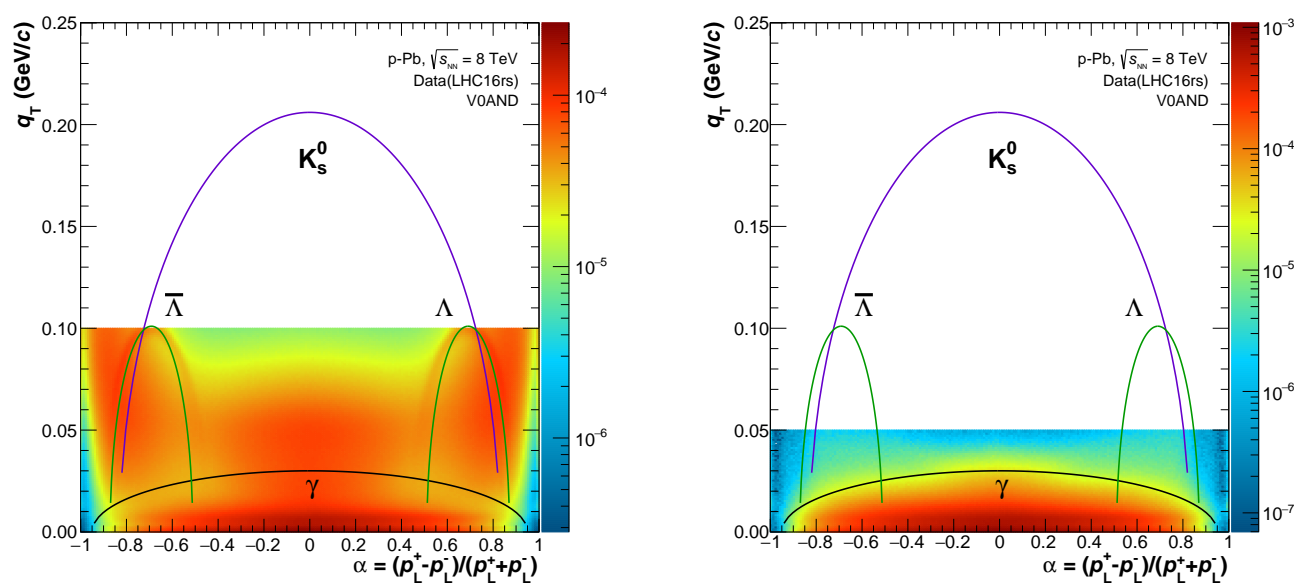

Figure 5.6: Left: Armenteros-Podolanski plot of $\mathrm{V}^{0}$ candidates that passed the track selection cuts in $\mathrm{p}-\mathrm{Pb}$ data at $\sqrt{s_{\mathrm{NN}}}=8.16 \mathrm{TeV}$. The data format used in the analysis already enforces a $q_{\mathrm{T}}<0.1$ preselection. Right: The distribution after all $\mathrm{V}^{0}$ candidate selection cuts are applied. 
In contrast, the heavy particles $\mathrm{K}_{S}^{0}$ and $\Lambda / \bar{\Lambda}$ lead to large opening angles of the daughters and therefore large values of $q_{\mathrm{T}}$. The momentum asymmetry $\alpha$ is distributed symmetric around values of zero due to the identical mass of the electrons and positions while one could easily distinguish $\Lambda$ from $\bar{\Lambda}$ due to the different charge of the heavier decay particle. In previous analyses, an elliptic cut (roughly indicated bu the black line in Figure 5.6) was applied in order to select the photon candidates from the $\mathrm{V}^{0}$ sample. This selection, however, rejected true high $p_{\mathrm{T}}$ photons from the sample and missed parts of the background at low $p_{\mathrm{T}}$. Therefore, optimized criteria are used where a $p_{\mathrm{T}}$ dependence of the $q_{\mathrm{T}}$ cut is applied as shown in Figure 5.7 with $q_{\mathrm{T}}<0.125 p_{\mathrm{T}}$ while still requiring $q_{\mathrm{T}}<0.05$ based on the true photon distribution. The cut on the asymmetry is loosened to $\alpha<0.95$ allowing for an increase in reconstruction efficiency compared to previous analyses. Overall, the reconstruction efficiency was increased by up to $15 \%$ with these optimized cuts while the pho-

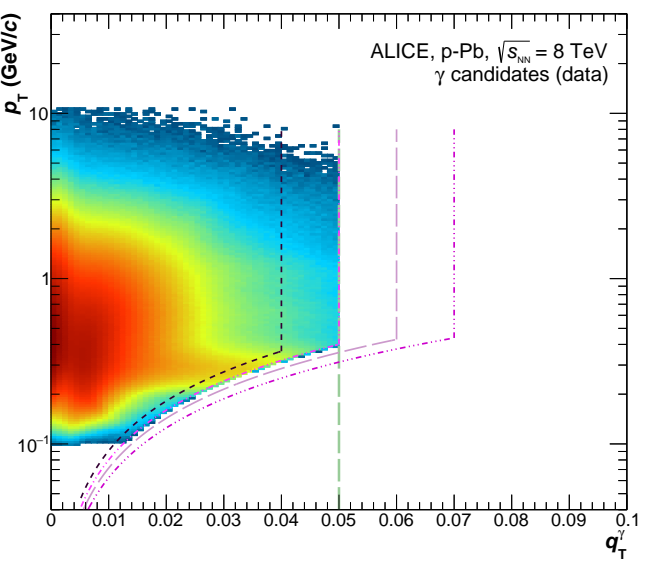

Figure 5.7: Two dimensional distribution of $q_{\mathrm{T}}$ versus $p_{\mathrm{T}}$. Distributions are shown after the standard cut for the analysis has been applied. Further cut variations used in the systematic uncertainty studies are indicated by dotted lines. ton purity reaches about $96 \%$.

\subsection{EMCal photon clusters}

Photons can be reconstructed using their energy deposit from electromagnetic showers induced in calorimeter material. This reconstruction technique is referred to as EMC for photons reconstructed with the EMCal and as PHOS for reconstruction with the PHOS detector. The following section covers the cluster reconstruction algorithm as well as the selection criteria for photon cluster candidates with the ALICE EMCal.

\subsubsection{Cluster reconstruction}

Photons hitting the EMCal induce an electromagnetic shower starting in a given cell. Depending on the particle incident angle as well as particle energy, the shower usually spreads to adjacent cells inducing further signals. However, most of the particle energy (on average about $70 \%$ ) is deposited in the leading cell that was directly hit. In order to reconstruct the full particle energy, the energy deposits in adjacent cells need to be added to the leading cell using a process called clusterization. In EMCal two main clusterization algorithms are used, the V1 and V2 clusterizers. Both clusterizers start with a seed cell which exceeds an energy threshold (in this analysis $E_{\text {seed }}=0.5 \mathrm{GeV}$ ). Adjacent cells with a common edge to this seed cell are added to the cluster if their energy surpasses an aggregation threshold (here $E_{\text {cell }}=0.1 \mathrm{GeV}$ ). This process of adding adjacent cells is repeated around each added cell until no further cell above the threshold is found. The difference between the V1 and V2 clusterizers lies within the condition to stop the aggregation. While the V1 will add all possible adjacent cells above threshold, the V2 will not add an adjacent cell whose energy is higher compared to the already clusterized reference cell. Based on these algorithms, a V2 cluster can only have one maximum while a V1 cluster can have several local maxima in the shower.

Another version of the V1 clusterizer, the V1 unfolding clusterizer can provide clusters similar to the V2 clusterizer by splitting the cluster based on the energy distributions around the local maxima. After the splitting, the energy of a certain cell can be split between multiple of the 


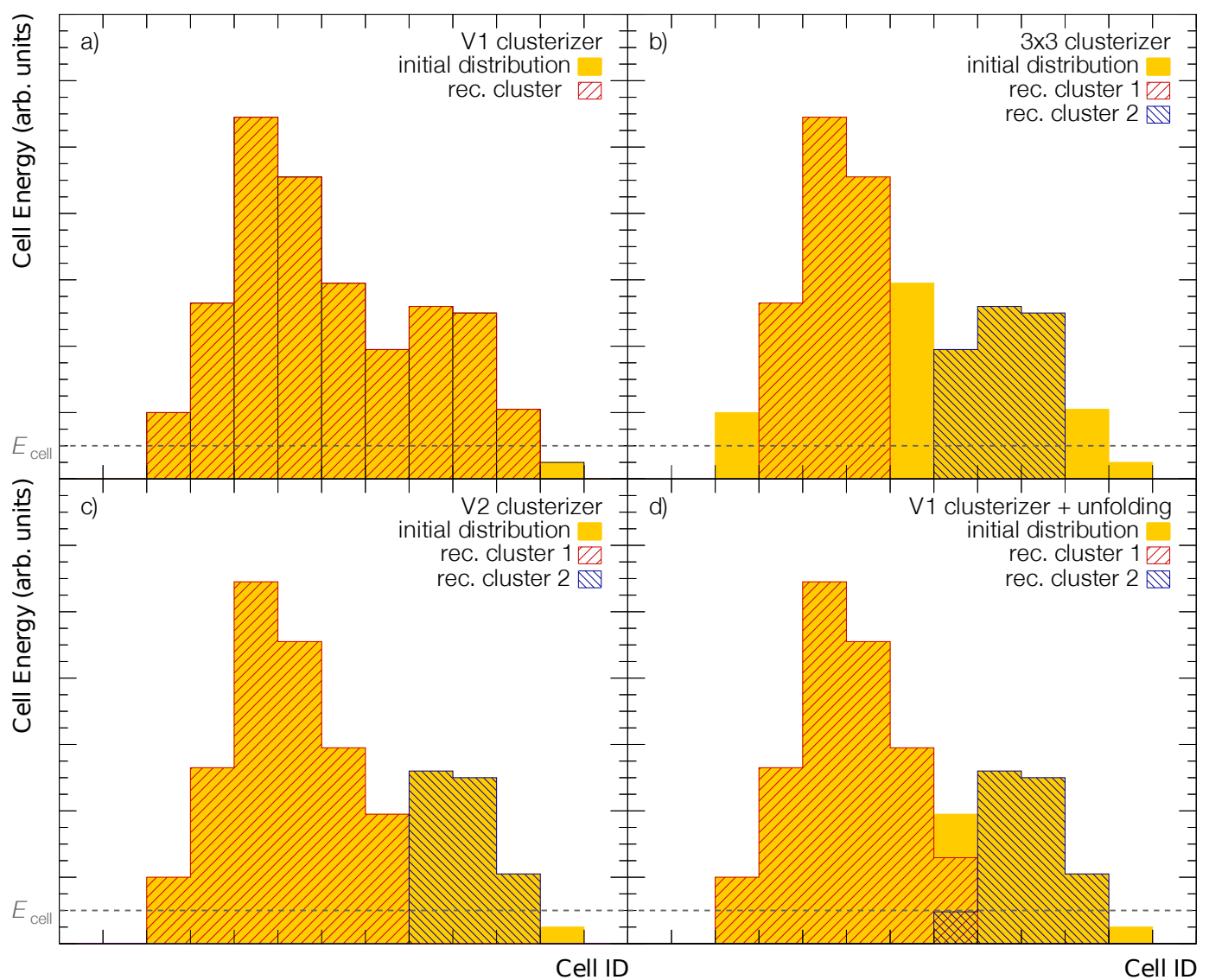

Figure 5.8: Illustrative visualization of the four different clusterization algorithms available for EMCal. Shown are V1 in a), $3 \times 3$ in b), V2 in c) and V1 unfolding in d). Indicated by yellow boxes are example energy deposits for adjacent cells in $\eta$ or $\phi$ direction on the calorimeter. The threshold for cell aggregation $E_{\text {cell }}$ is indicated with a gray dotted line. Adapted from [150].

unfolded clusters. This can theoretically provide a better energy resolution for overlapping particle showers compared to the V2 clusterizer which uses a sharp cut-off.

A fourth clusterizer $(\mathrm{N} \times \mathrm{N})$ can be used with the EMCal, which sums energy deposits in an array of cells, usually $3 \times 3$, around a leading cell above the clusterization threshold. This clusterizer performs well in low density environments and requires the least computing time of all discussed algorithms. It has been used in the EMCal test beam studies, but was not considered in the presented analyses due to it's reduced performance in high density environments, like high multiplicity $\mathrm{p}-\mathrm{Pb}$ collisions. The behavior of all four clusterizers for an example energy distribution in adjacent cells is shown illustrative in Figure 5.8. Despite comparable performance of these clusterizers, it has been found in previous analyses that the V2 clusterizer is best suited for particle measurements involving two-photon decay channels. This was concluded as the resulting cluster properties are better reproduced in simulations pointing to a lower sensitivity to shower overlaps from other particles in high multiplicity environments. In the following analyses, the V2 clusterizer is therefore chosen as the default clusterizer. In order to reduce contributions from out-of-bunch pileup, strict timing cuts are applied on the reconstructed cluster times around the main bunch crossing based on the bunch spacing during the given data taking period.

The cluster time distributions for $\mathrm{pp}$ and $\mathrm{p}-\mathrm{Pb}$ data are shown in Figure 5.9 where the clusters coming from different bunch crossings are visible as equidistant peaks. The bunch crossings closest to the main peak in the $\mathrm{p}-\mathrm{Pb}$ data are largely rejected by the past-future rejection applied in the event selection which is reflected by the full suppression of the expected peak at 
100 ns. The past-future rejection is not available for pp data, as the necessary algorithms were not yet implemented during data taking campaign in 2012. It was introduced to better deal with pileup removal in the high luminosity environment during the LHC Run 2. For pp $\sqrt{s}=8 \mathrm{TeV}$ the bunch spacing was $25 \mathrm{~ns}$, so a timing cut of about $\pm 30 \mathrm{~ns}$ was applied while for $\mathrm{p}-\mathrm{Pb} \sqrt{s_{\mathrm{NN}}}=8.16 \mathrm{TeV}$ a looser cut of \pm 50 ns could be used due to a much larger bunch spacing. As timing information as well as out-of-bunch pileup is not simulated in ALICE, the timing cuts are only applied in data. Moreover, for the cells entering the clusterization process an additional timing cut was en-

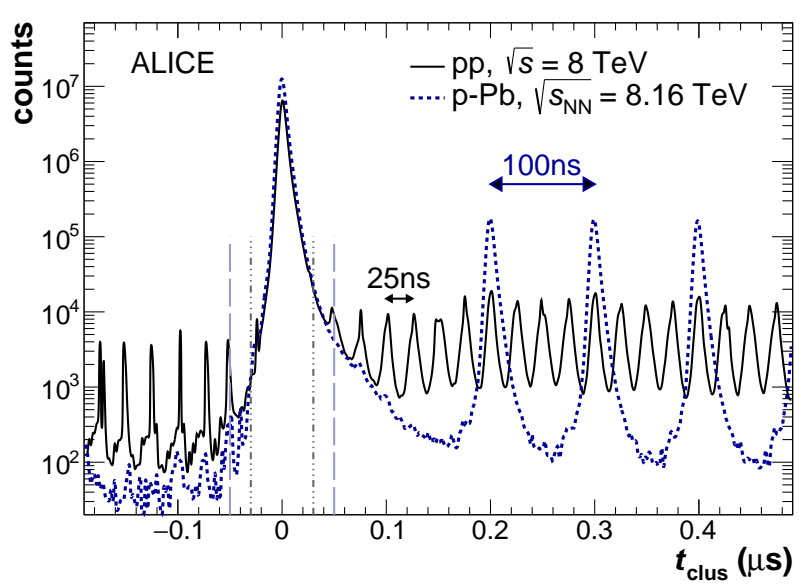

Figure 5.9: Cluster time and bunch spacing in $\mathrm{pp}$ (black) and $\mathrm{p}-\mathrm{Pb}$ (blue) data.

forced of $\pm 500 \mathrm{~ns}$ which is rather loose due to the poor timing resolution at very low cell energies where a tighter cut would remove actual signal in data and induce a bias in the measurement.

\subsubsection{Photon cluster selection}

In order to select photon candidates from the V2 clusterizer cluster sample, several selection criteria are applied as listed in Table 5. The selection begins with the clusterizer settings requiring a seed energy of $500 \mathrm{MeV}$ and a minimum cluster energy of $700 \mathrm{MeV}$. With this, minimum ionizing energy loss signals from charged particles are strongly suppressed. These signals have been determined to be about $236 \mathrm{MeV}$ based on a Landau-Gaussian fit on testbeam data from muon and hadron beams [150]. EMCal clusters are additionally required to be made up of at

\begin{tabular}{lll}
\hline Cluster selection criteria & \\
\hline Clusterizer & algorithm & V3 $(\mathrm{V} 2$ with optimized algorithm $)$ \\
& min. seed energy $E_{\text {seed }}$ & $0.50 \mathrm{GeV}$ \\
& min. cell energy $E_{\text {cell }}$ & $0.10 \mathrm{GeV}$ \\
& cell timing & $\left|t_{\text {cell }}\right|<500 \mathrm{~ns}$ \\
\hline Acceptance & pseudorapidity & $|\eta|<0.67$ \\
& azimuth & $1.4<\varphi<3.28(\mathrm{EMCal})$ \\
& & $4.56<\varphi<5.70(\mathrm{DCal})$ \\
\hline Quality & min. cluster energy $E_{\min }$ & $>0.7 \mathrm{GeV}$ \\
& min. \# of cells & $N_{\text {cluster }}^{\text {cells }} \geq 2$ \\
& exotics removal & $F_{+}<0.97$ \\
& cluster time & $-30 \mathrm{~ns} \leq t_{\text {cluster }}<35 \mathrm{~ns}(\mathrm{pp} \sqrt{s}=8 \mathrm{TeV})$ \\
& shower shape parameter & $\left|t_{\text {cluster }}\right|<50 \mathrm{~ns}\left(\mathrm{p}-\mathrm{Pb} \sqrt{s_{\mathrm{NN}}}=8.16 \mathrm{TeV}\right)$ \\
& & $\sigma_{\text {long }}^{2}>0.2(\mathrm{mEMC})$ \\
& track matching & $|\Delta \eta|<0.010+\left(p_{\mathrm{T}}+4.07\right)^{-2.5}$ \\
& & $|\Delta \varphi|<0.015+\left(p_{\mathrm{T}}+3.65\right)^{-2}$ \\
& & $E_{\text {clus }} / p_{\text {track }}<1.75$ \\
\hline
\end{tabular}

Table 5: EMCal clusterization and photon candidate selection criteria used in the EMC, PCMEMC and mEMC analyses. 
least two cells in order to suppress noise in the readout electronics or signals from neutrons directly hitting the APD which will only induce a high energetic signal in a single cell. The latter is called an exotic cluster in the following. Exotic clusters are furthermore removed by requiring the leading cell to contain less than $97 \%$ of the cluster energy via a cut on the exoticity $F_{+}=1-E_{+} / E_{\text {cell }}^{\max }<0.97$. This cut has been determined from comparisons of data with $\mathrm{MC}$ simulations where neutron hits on APDs are not simulated and the respective distributions for data and MC are shown in Figure 5.10. A clear rise in data towards higher values of $F_{+}$is observed which is associated with the exotic cluster

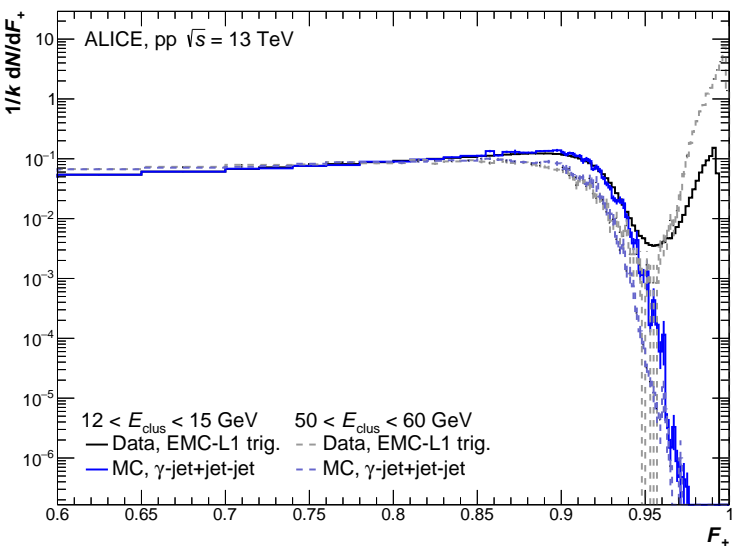

Figure 5.10: Exoticity of clusters in two energy ranges for EMCal L1 triggered data and Jet-Jet MC simulation [150]. signal. A neutralization criterium is applied on clusters in the form of a track matching procedure. This procedure rejects clusters where a track propagated to the average shower depth of EMCal (about $440 \mathrm{~cm}$ ) lies within a certain $|\Delta \eta|$ or $|\Delta \varphi|$ window depending on the track momentum as given in Table 5. In addition, if the condition $E_{\text {cls }} / p_{\text {track }}<1.75$ is fulfilled, the cluster rejection based on the given track is vetoed. This condition is especially important at high cluster energies where a random low $p_{\mathrm{T}}$ track could be matched to a cluster which would lead to the unwanted removal of the cluster. The addition of this condition improved the reconstruction efficiency at high $p_{\mathrm{T}}$ by up to $50 \%$ at $200 \mathrm{GeV}$ with negligible effects on the cluster purity. The track matching procedure is also important for the PCM-EMC neutral meson reconstruction technique (explained in Section 6.1) where a conversion electron cluster could otherwise be combined with the conversion photon leading to autocorrelations [88]. The removal of the $\mathrm{V}^{0}$ tracks is done with an efficiency of more than $99 \%$ due to the fact that the tracks are well constrained by the $\mathrm{V}^{0}$ conditions. One of the most important selection criteria for photon identification is applied on the shower shape in the $\eta-\varphi$ plane which can be characterized by the eigenvalue squared based on the energy distribution in this plane [199]. The shower shape long axis $\sigma_{\text {long }}^{2}$ is defined as

$$
\sigma_{\text {long }}^{2}=\frac{1}{2}\left(\sigma_{\varphi \varphi}^{2}+\sigma_{\eta \eta}^{2}+\sqrt{\left(\sigma_{\varphi \varphi}^{2}-\sigma_{\eta \eta}^{2}\right)^{2}+4 \sigma_{\varphi \eta}^{4}}\right)
$$

where the values of $\sigma_{a b}^{2}=\langle a b\rangle-\langle a\rangle\langle b\rangle$ and $\langle a\rangle=$ $\left(w_{\text {tot }}\right)^{-1} \sum w_{i} a_{i}$ are based on the weighted cell energy compared to the cluster energy as well as in relative $\eta$ and $\phi$ direction to the leading cell. The weighting is $\operatorname{logarithmic}$ with $w_{i}=\max \left(0,4.5+\log \left(E_{i} / E_{\text {clus }}\right)\right.$ where the sum of all $w_{i}$ equals $w_{\text {tot }}[199] . \quad \sigma_{\text {long }}^{2}$ is used as an additional criterium to reject exotic clusters with a minimum cut requiring $\sigma_{\text {long }}^{2}>0.1$ as very small shower shape values are not expected for higher energetic clusters due to the spread of the electromagnetic shower over many adjacent cells. For photon identification, an additional maximum cut of $\sigma_{\text {long }}^{2}<0.5$ is applied in order to reject largely elliptic clusters which either originate from non-resolvable overlapping showers or from electrons entering the EMCal with a strong inclination

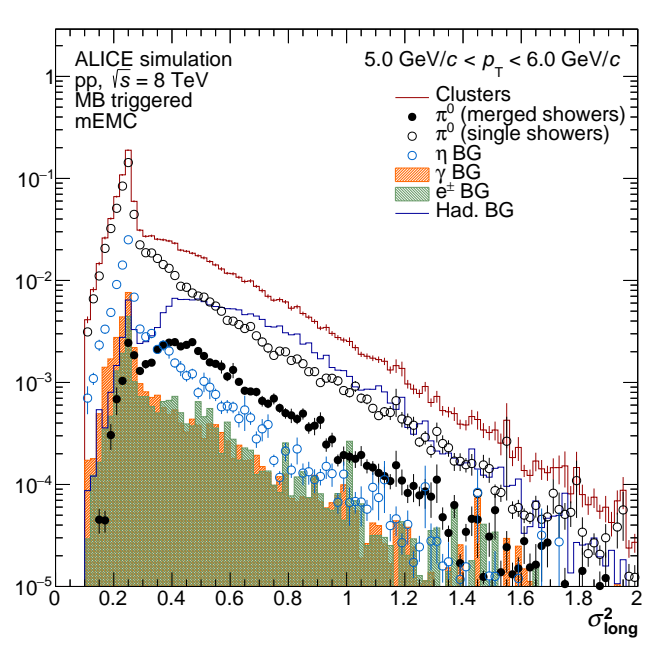

Figure 5.11: Example $\sigma_{\text {long }}^{2}$ distribution from simulation highlighting the different particle contributions. 
due to the magnetic field. This maximum cut is in addition chosen such, that is still allows to capture clusters from late conversions where both electrons are contained. As photons are unaffected by the magnetic field they mainly hit the EMCal (aside from the large $\eta$ regions) perpendicular and induce a shower with circular shape in the $\eta-\varphi$ plane. The average shower shape value for photons is around $\sigma_{\text {long }}^{2} \approx 0.25$ as seen in Figure 5.11 while charged hadrons or electrons are shifted systematically to larger values. For the merged cluster $\pi^{0}$ analysis (see Section 6.2) the photon peak is mainly considered background and therefore a default minimum cut of $\sigma_{\text {long }}^{2}>0.27$ is applied in this analysis. In addition, the merged pion analysis only considers clusters with energies of $E_{\text {clus }}>15 \mathrm{GeV}$ due to the decay kinematics of the $\pi^{0}$ combined with the detector resolution.

\subsubsection{EMCal bad channel maps}

A fraction of EMCal cells produce a bad time or energy response signal for incoming particle hits. Such cells need to be excluded from the analysis as MC simulations are not designed to reproduce such effects. Bad cells can be subdivided into groups of hot, cold or dead cells depending on their response compared to the expected signal distribution. Cells are considered hot if they constantly produce a signal even if they are not hit or if they produce unreasonably large signals. Such cells are easily found by comparing their energy signal spectrum to a reference spectrum of known good cells. A strong difference in

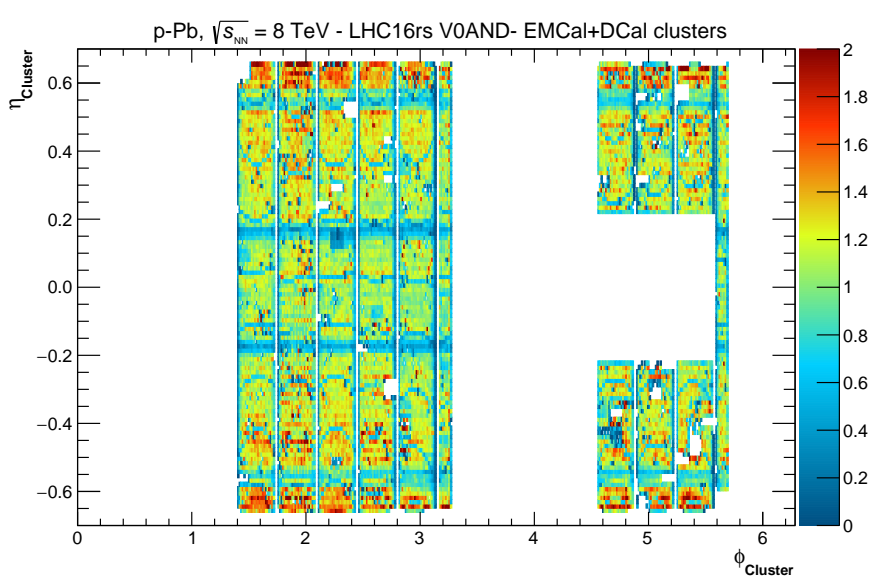

Figure 5.12: $\eta-\phi$ projection of clusters in EMCal and DCal. Deactivated channels and module borders as well as the PHOS hole are visible. shape or a peak in the distribution at larger energies is used to flag those cells as hot and therefore as bad. For cold cells we see only small signals which are often visible as a steeply falling energy spectrum where no high energetic hits can be found. Lastly, dead cells register no hits and can therefore be automatically detected and excluded. In total about $6 \%$ of EMCal cells are masked which also contains those parts of the detector that were deactivated during data taking due to noise. Figure 5.12 shows the projection of all cluster positions in the $\eta-\varphi$ plane in $\mathrm{p}-\mathrm{Pb}$ data where masked bad channels are visible as white space in the modules. Furthermore, the module borders are visible between all 20 supermodules as well as the presence of the TRD support structures in front of EMCal which suppress particle hits in certain areas (visible in the horizontal blue lines). The projection also shows the geometry of the EMCal and the DCal, accurately visualizing the gap in the pseudorapidity coverage between the first six of the DCal modules where the PHOS detector is located.

\subsubsection{EMCal cell time calibration}

The time signal of cells in EMCal requires careful calibration in order to allow for strict time cuts to remove out-of-bunch signals. It is affected by the length of the cables used to read out the detector which approximates to $\approx 600 \mathrm{~ns}$ of signal delay as well as by effects of the readout electronics. Additionally, an alignment between the EMCal ALTRO and the LHC clocks is performed due to the difference in bunch spacing relative to the 100ns sampling of the detector hardware. The time calibration is performed on cells with energies of $E_{\text {cell }}>0.4 \mathrm{GeV}$ in order 
to ensure a proper resolution of the cells timing information. The cell time distributions are then shifted by the correction offsets for the cable length and clock difference in order to have their main peak centered around 0 ns. After the calibration, a timing resolution of less than $3 \mathrm{~ns}$ is achieved. Figure 5.9 shows the cell times of the leading cell in clusters found in EMCal, visualizing the main peak centered around 0 ns with repeating peaks at each subsequent (and previous) bunch crossing.

\subsubsection{Energy nonlinearity and scale}

The EMCal, like most calorimeters, provides a nonlinear response between the incoming and reconstructed energy. This effect originates from the calorimeter design as well as its hardware and can be split into several components which affect the readout. The main components are nonlinearities in the detector hardware, effects due to the hit position of the particle relative to the cell center as well as energy loss in the shower between cells. In addition, particle energy losses due to the material budget in front of EMCal affect the reconstructed energy as well. These effects are corrected for in three steps starting

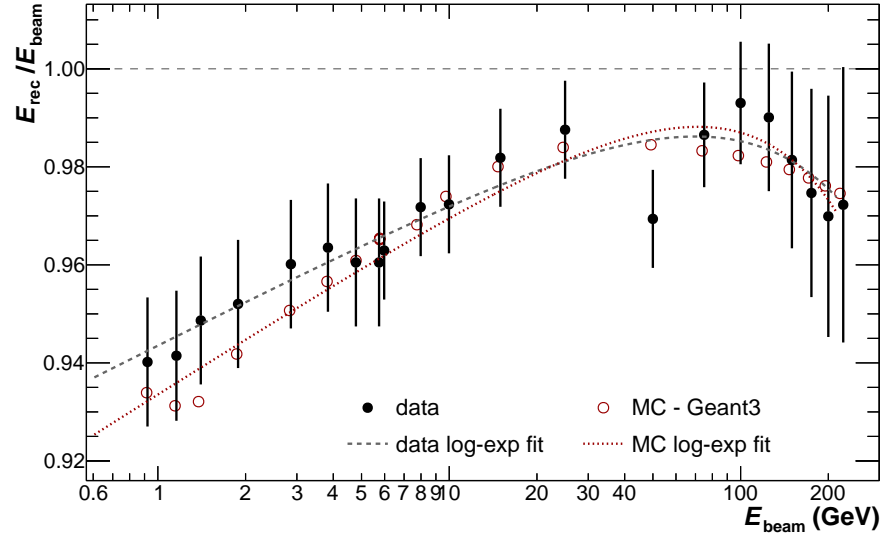

Figure 5.13: Energy and position nonlinearity correction from electron testbeam data (black) and corresponding GEANT3 MC simulations (red) together with the log-exp fits [150]. with studies on the EMCal test beam setup [200,201] at the Proton Synchrotron (PS) and the Super Proton Synchrotron (SPS) where a test setup of $8 \times 8$ EMCal cells was used together with multi-wire proportional chamber (MWPC) tracking detectors in order to determine the response to incident electron and hadron beams at varying energies. The test setup was calibrated for energy and temperature and data with beam energies from $750 \mathrm{MeV}$ up to $230 \mathrm{GeV}$ was recorded. The high energetic electron beam data $E_{\text {beam }}>100 \mathrm{GeV}$ showed a significant nonlinear response which was determined in the laboratory to originate from the shapers on the front end electronics (FEE). The nonlinearity was parametrized with a sixth order polynomial resulting in about $2 \%$ missing energy at 100 $\mathrm{GeV}$ and $13 \%$ missing energy at $E_{\text {cell }}=150 \mathrm{GeV}$ strongly increasing for higher energies. This correction is applied on analysis level on each cell with $E_{\text {cell }}>16 \mathrm{GeV}$ before clusterization.

In a next step, the calorimeter response due to the hit position in the cells, the light collection efficiency and the shower leakage are corrected for by comparison of the incident beam energy with the reconstructed cluster energies as a function of beam energy as shown in Figure 5.13. The data shows a necessary correction of about $6 \%$ at low energies while for $E_{\text {beam }}>20 \mathrm{GeV}$ the correction is in order of $2-3 \%$. Simulations are able to describe the data on a percent level and both data and MC are fitted with a functional form given by

$$
f(E)=\frac{p_{0}+p_{1} \ln (E)}{1+p_{2} \exp \left[\left(E-p_{3}\right) / p_{4}\right]}
$$

which is able to describe the low and high energy region within uncertainties. The parametrizations according to Equation 21 which are shown in Figure 5.13 are used to correct the reconstructed cluster energies in data and $\mathrm{MC}$ on analysis level. With these nonlinearity corrections applied, the data and MC simulations are checked for residual differences which are attributed mainly to the material budget in front of EMCal. The material, composed of the TRD and 


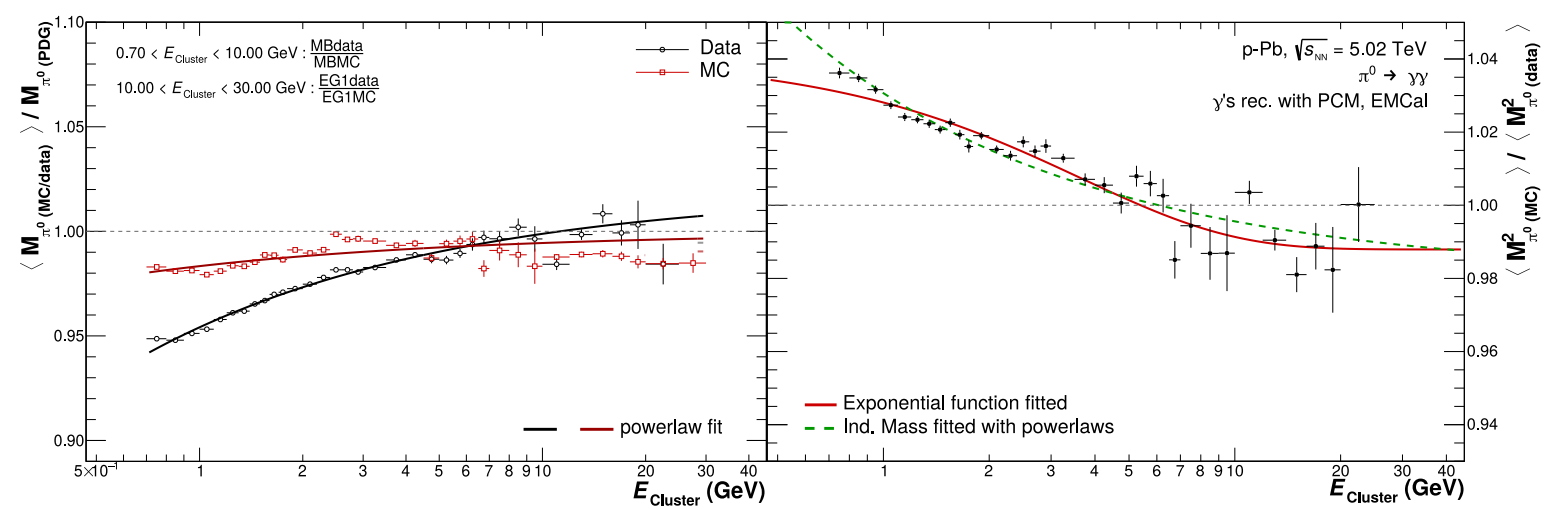

Figure 5.14: Left: $\pi^{0}$ invariant mass peak positions in data (black) and simulation (red) relative to the nominal PDG mass together with power-law fits. Right: Ratio of the squared $\pi^{0}$ invariant mass peak positions in simulation and data together with ratios of the respective power-law fits as well as with a separately fitted exponential ratio function.

TOF detectors as well as support structures, amounts to a combined radiation length of about $0.3 X / X_{0}$ and is responsible for large amounts of photon conversions that smear and reduce the reconstructed cluster energies. Figure 5.14 shows the $\pi^{0}$ meson invariant mass peak positions in data and simulations versus cluster energy in $\mathrm{p}-\mathrm{Pb}$ collisions at $\sqrt{s_{\mathrm{NN}}}=5.02 \mathrm{TeV}$. This dataset was chosen for determining the correction factor as it provides an order of magnitude more statistics in the minimum bias data set, thus reducing uncertainties. It has been verified that the correction factors in all LHC Run 2 data recorded by ALICE are consistent within uncertainties. For the correction procedure, $\pi^{0}$ mesons are reconstructed with a hybrid PCM-EMC approach where PCM photons are combined with EMCal photon candidates in order to calculate the invariant mass. This allows to profit from the high momentum resolution of the PCM photon and circumvents effects of cluster merging that would appear for $E_{\text {clus }}>6 \mathrm{GeV}$ when using two EMCal photons instead. The invariant mass $m_{\gamma \gamma}$ is calculated from the energies of the respective photons $E_{\gamma_{1,2}}$ and the opening angle between both photons in the laboratory frame via

$$
m_{\gamma \gamma}=\sqrt{2 E_{\gamma_{1}} E_{\gamma_{2}}\left(1-\cos \theta_{12}\right)} .
$$

An excess around the $\pi^{0}$ as well as around the $\eta$ meson invariant mass becomes visible in the resulting invariant mass distribution around $m_{\gamma \gamma}=135 \mathrm{MeV} / c^{2}$ and $m_{\gamma \gamma}=548 \mathrm{MeV} / c^{2}$, respectively. The peak positions shown in Figure 5.14 are obtained from Gaussian fits on the invariant mass distributions in slices of cluster energy where the fitting range is restricted to only include the region within the FWHM of the peak. This way, exponential tails on the left or right side of the peak from electron bremsstrahlung or shower overlaps and smearing are largely excluded from the fitting procedure. A residual difference between data and simulation can be seen while in both cases the reconstructed $\pi^{0}$ mass lies below the PDG mass. The difference is better visualized in the right panel of the same figure where the ratio of the squared masses in simulation and data is given. This shows an approximate $3 \%$ higher invariant mass in simulation compared to data at low energies while at high energies simulations underestimate the data by about $1 \%$. In order to obtain the correction factor, the ratio in the right panel is fitted with an exponential function defined as

$$
f_{\text {ratio }}=p_{0}+\exp \left(p_{1}+p_{2} E_{\text {cluster }}\right) .
$$

The resulting correction function is used on analysis level to correct the MC simulation cluster energies in order to bring them into agreement with data. In this procedure, data is therefore 


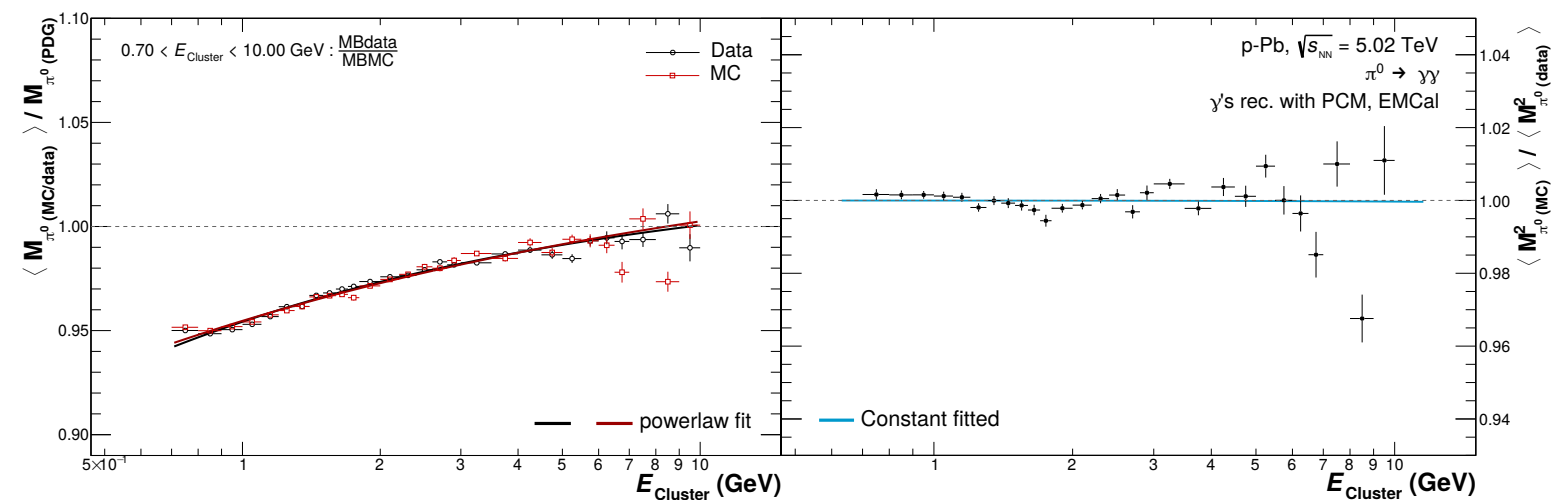

Figure 5.15: Left: $\pi^{0}$ invariant mass peak positions in data (black) and simulation including fine tuning correction (red) relative to the nominal PDG mass together with power-law fits. Right: Ratio of the squared $\pi^{0}$ invariant mass peak positions in simulation and data together with a constant fit function.

considered as "truth" and the data clusters are not modified by the correction. The invariant mass peak positions and their squared ratio after the correction factor has been applied in simulation can be seen in Figure 5.15. A per-mille level agreement between data and MC is achieved with this procedure resulting in a minimal systematic uncertainty due to the energy scale as described in Section 6.3.

\subsubsection{Cross-talk emulation}

The shower shape distribution according to Equation 20 is sensitive to the energy distribution within the clusters. A discrepancy between data and MC has been found early on in analyses where an excess of low invariant mass signals appeared in $\pi^{0}$ analyses. This was attributed to a hardware-level effect, called cross-talk, where a signal induced in a given EMCal tower affects the baseline of surrounding towers within the same T-Card [199]. Such a baseline change is the main reason for a significant broadening of the $\sigma_{\text {long }}^{2}$ distribution in data compared to $\mathrm{MC}$ which is especially observed for supermodules 3 and 7 of the EMCal. An emulation for this cross talk induced distribution of energy was developed for Monte Carlo simulations considering that cross talk affects all cells, that it can affect even cells that are two rows apart and that there is a possible cell energy and supermodule dependence. The cross talk furthermore does not induce additional signal, it is just a re-distribution of a fraction of the selected cell energy into surrounding cells.

For full jet measurements, this effect is therefore less relevant as it conserves the total energy when inspecting large areas on the EMCal surface. It

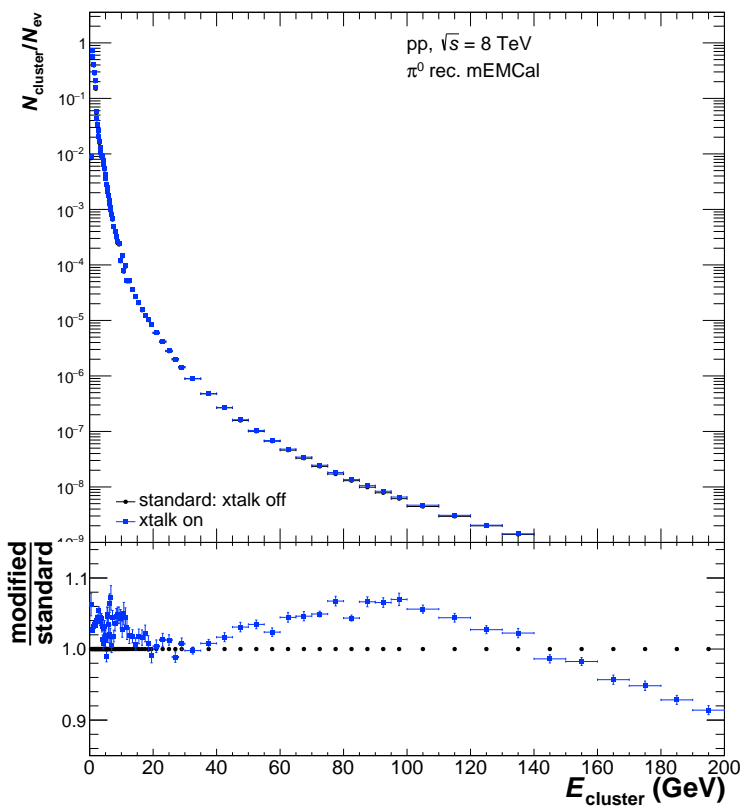

Figure 5.16: Cluster energy spectrum for clusters with $\sigma_{\text {long }}^{2}>0.2$ with and without cross talk emulation in pp collisions at $\sqrt{s}=8$ $\mathrm{TeV}$. 

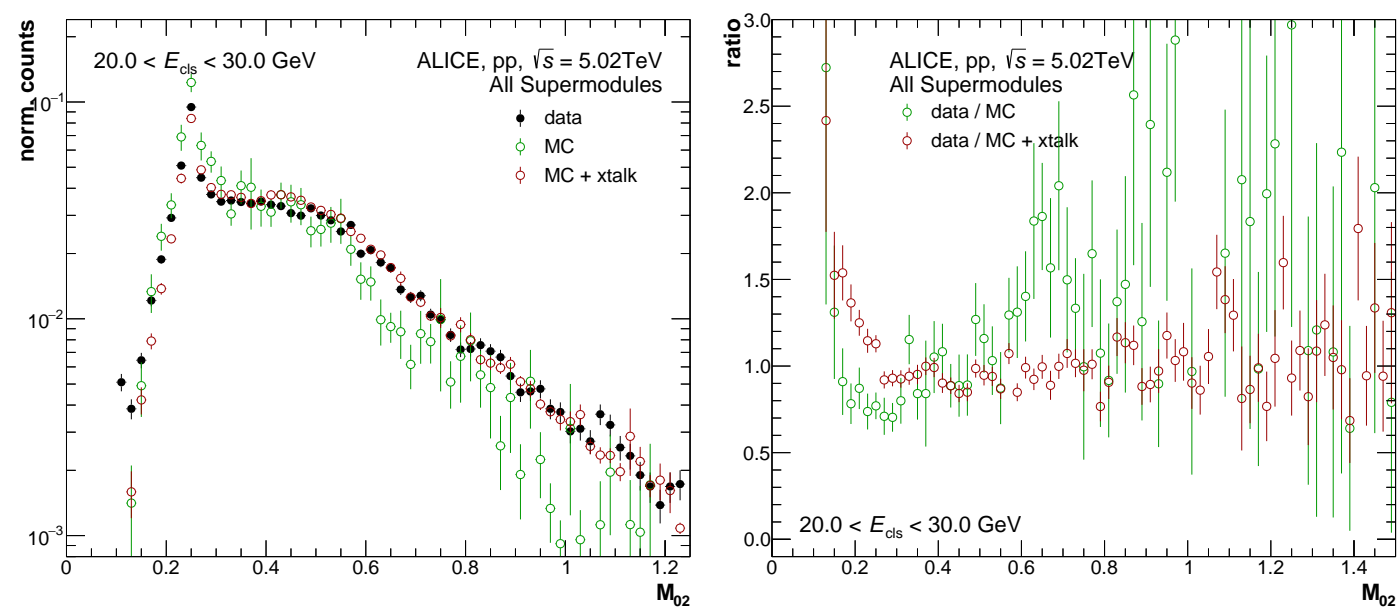

Figure 5.17: Left: Shower shape distribution $\left(\sigma_{\text {long }}^{2}\right)$ in data and Monte Carlo simulations with and without the cross talk emulation in pp collisions at $\sqrt{s}=5.02 \mathrm{TeV}$. Right: Ratio of the $\sigma_{\text {long }}^{2}$ distributions of data and Monte Carlo simulations with the cross talk emulation and without it.

tribution of energy within a given cluster is important [202]. From detailed studies on pp collision data and simulations at $\sqrt{s}=7$ and $13 \mathrm{TeV}$ it was determined that about $0.35-1.8 \%$ of the cell energy is distributed to surrounding cells due to cross talk. A comparison of the shower shape distribution with and without the cross talk emulation can be found in Figure 5.17. A significant improvement especially for the $\sigma_{\text {long }}^{2}>0.2$ region where instead of an up to $50 \%$ discrepancy one now has a $\pm 5 \%$ agreement between data and $\mathrm{MC}$ in the shower shape. The effect on the cluster spectrum in simulations for clusters with $\sigma_{\text {long }}^{2}>0.2$ (as used in Section 6.2 for the merged cluster analysis) is shown in Figure 5.16. An up to $10 \%$ effect on the cluster energy spectrum can be seen with a change in the overall spectral slope for $E_{\text {clus }}>100 \mathrm{GeV}$ which affects the reconstruction efficiency of these clusters in a similar way. The emulation of cross talk is one of the key ingredients for single cluster analyses with EMCal where a shower shape cut is applied. 


\section{Meson Measurements}

In this chapter the neutral pion and $\eta$ meson analyses in pp collisions at $\sqrt{s}=8 \mathrm{TeV}$ and $\mathrm{p}-\mathrm{Pb}$ collisions at $\sqrt{s_{\mathrm{NN}}}=8.16 \mathrm{TeV}$ are described. They present in both collision systems the highest ever measured identified particle spectra with $\pi^{0}$ meson $p_{\mathrm{T}}$-differential invariant cross sections and nuclear modification factors reaching transverse momenta of $200 \mathrm{GeV} / c$. With such a $p_{\mathrm{T}}$ reach, this exceed previous $\pi^{0}$ measurements by nearly an order of magnitude $[195,196]$. The same measurements are performed for the $\eta$ meson, reaching in $\mathrm{p}-\mathrm{Pb}$ transverse momenta of up to $p_{\mathrm{T}}=50 \mathrm{GeV} / c$. The measurements are performed either based on a statistical invariant mass based approach using the photons reconstructed with PCM and EMC as described in the last section or using a particle identification approach for the high $p_{\mathrm{T}}$ measurement via the shower shape of overlapping (merged) photon clusters. In total, up to five reconstruction techniques are used for the $\pi^{0}$ andthe $\eta$ meson measurements. In these techniques, either both photons for the invariant mass calculation are taken from the same photon detection method (called PCM, EMC and PHOS) or a conversion photon is mixed with a calorimeter photon in the PCM-EMC hybrid approach. The PHOS measurements were provided independently from the presented analyses and are combined with the results in both collision systems [196,203,204]. For all techniques besides PCM, triggered data is used to extend the spectra to higher momenta, combining in total data from four event triggers for the measurements.

The highlight of these measurements is the merged EMCal (mEMC) analysis which has been refined and, due to a highly improved understanding of the method and the detector response, extended to unprecedented $p_{\mathrm{T}}$ as described in Section 6.2. The general method itself has previously been used in a neutral pion measurement in pp collisions at $\sqrt{s}=2.76 \mathrm{TeV}$ [195] where it extended the invariant mass-based spectra up to $40 \mathrm{GeV} / c$.

With the measurements of the $\pi^{0}$ and $\eta$ meson, the $\eta / \pi^{0}$ ratio is also determined up to $50 \mathrm{GeV} / c$ in $\mathrm{p}-\mathrm{Pb}$ and $35 \mathrm{GeV} / c$ in $\mathrm{pp}$, exploiting the additional mEMC spectrum for a higher $p_{\mathrm{T}}$ reach. Lastly, the cross section measurements in $\mathrm{pp}$ and $\mathrm{p}-\mathrm{Pb}$ are combined for the nuclear modification factor $\left(R_{\mathrm{pA}}\right)$ measurement up to the same high transverse momenta.

The first sections in the following will introduce the meson reconstruction techniques, where several components have been optimized compared to previous analyses. Afterwards, the correction factors are introduced which is followed by an overview of the systematic uncertainty determination procedures. In a last step, the spectra of all individual reconstruction techniques are combined into the final results for the various spectra. The results are then compared to theoretical predictions of next-to-leading order pQCD as well as PYTHIA8.2 calculations. The chapter is concluded with a summary of the presented measurements and an outlook to new measurements motivated by these results is given.

\subsection{Reconstruction via invariant mass}

The neutral mesons $\left(\pi^{0}\right.$ and $\left.\eta\right)$ can be reconstructed from their two-photon decay channel, with branching ratios of $\mathrm{BR}_{\pi^{0} \rightarrow \gamma \gamma}=98.82 \%$ and $\mathrm{BR}_{\eta \rightarrow \gamma \gamma}=39.41 \%$ [19], via excess yield in the invariant mass $\left(M_{\gamma \gamma}\right)$ distribution. $M_{\gamma \gamma}$ is calculated according to

$$
M_{\gamma \gamma}=\sqrt{2 E_{\gamma_{1}} E_{\gamma_{2}}\left(1-\cos \theta_{12}\right)}
$$

where the photon energies are given by $E_{\gamma_{1}}$ and $E_{\gamma_{2}}$ and the opening angle between both photons in the laboratory frame by $\theta_{12}$ as previously described in Section 5.2.3. While the photon candidates are either reconstructed from photon conversions (PCM) or from energy deposits in a calorimeter (EMC, PHOS), the neutral meson candidates can be formed from either two photon 

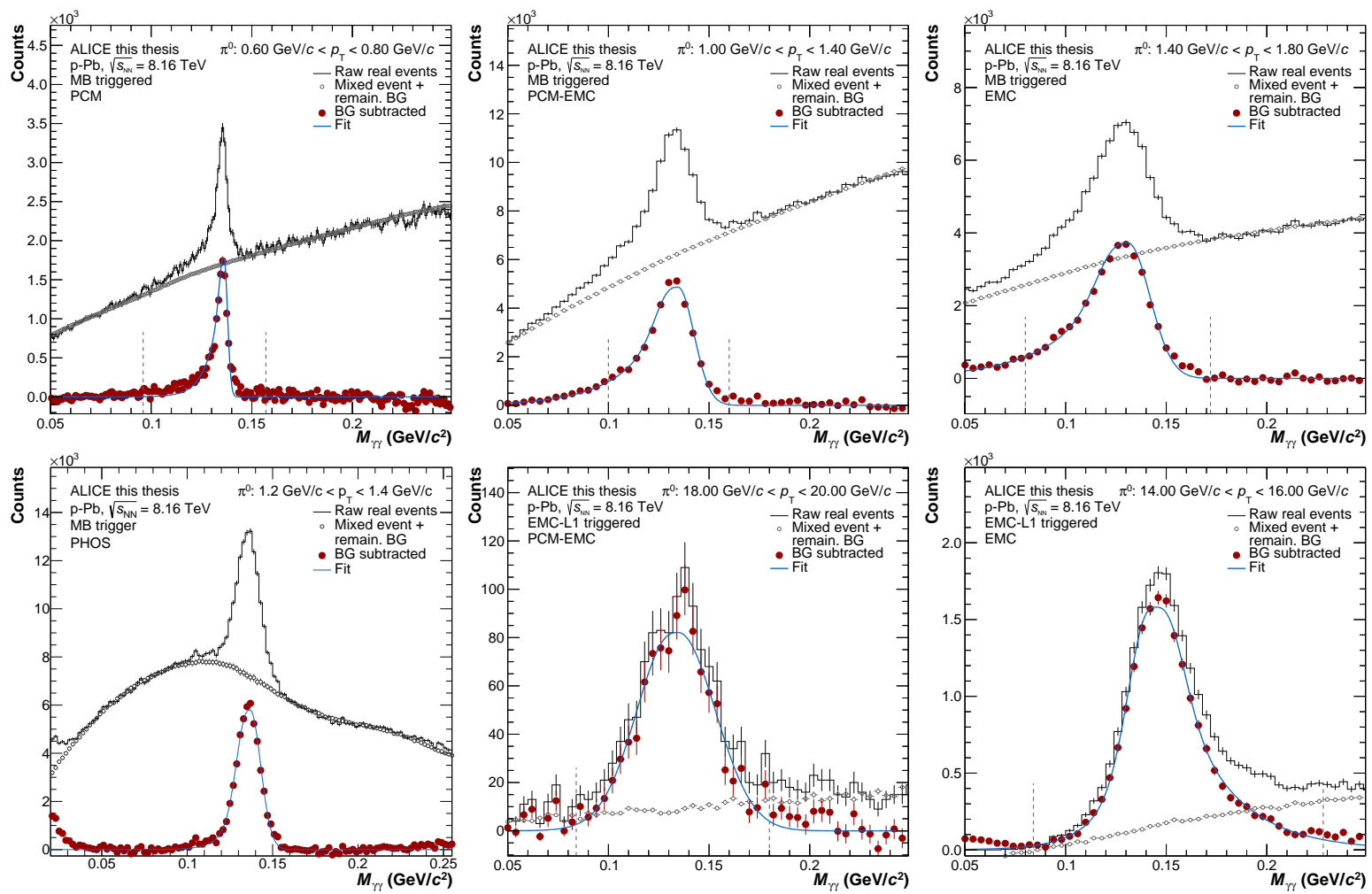

Figure 6.1: Invariant mass distributions in a wide window around the nominal $\pi^{0}$ meson mass for minimum bias data of $\mathrm{p}-\mathrm{Pb}$ collisions at $\sqrt{s_{\mathrm{NN}}}=8.16 \mathrm{TeV}($ top $)$ and triggered data (bottom) for PCM (top left), PCM-EMC (middle), EMC (right) and PHOS (bottom left).

candidates of the same reconstruction technique or via a hybrid approach, where a PCM photon is paired with a calorimeter photon, called PCM-EMC. A PCM-PHOS hybrid approach was not feasible in the presented data sets due to statistical limitations.

The invariant mass distributions are then calculated in a rapidity window of $|y|<0.8$ via combination of all possible photon candidate pairs for the respective methods in each event. Example distributions are shown in Figure 6.1 in the vicinity of the nominal neutral pion mass and in Figure 6.2 of the $\eta$ meson mass in the $\mathrm{p}-\mathrm{Pb} \sqrt{s_{\mathrm{NN}}}=8.16 \mathrm{TeV}$ data. Similar example distributions for the pp $\sqrt{s}=8 \mathrm{TeV}$ data are given in Figure A.1 and Figure A.2 for the $\pi^{0}$ and $\eta$, respectively. A clear excess is visible in all distributions close to the nominal meson mass of $135 \mathrm{GeV} / c^{2}$ for the $\pi^{0}$ and $548 \mathrm{GeV} / c^{2}$ for the $\eta$ meson. The peak is positioned on top of a combinatorial background distribution, which is substantial especially at low transverse momenta. The underlying combinatorial background needs to be subtracted and is calculated based on an event mixing technique [205]. Within this technique, photon candidates from an event pool based on the primary vertex position as well as the photon multiplicity are mixed with photons from the current event. The pool is set to contain 80 photon candidates from previous events binned into four sub-pools based on photon or charged particle multiplicity and seven sub-pools based on the $z$ position of the primary vertex in order to only mix events with similar event properties. The resulting event-mixed invariant mass distribution is by construction correlation free and therefore does not show excess yield around the neutral meson masses. It is scaled to the right side of the peak as shown in the open black points in Figure 6.1 and Figure 6.2. For the same and mixed event invariant mass calculation only photon candidate pairs with a minimum opening angle of $\theta_{\min }>5 \mathrm{mrad}$ for PCM and PCM-EMC and $\theta_{\min }>17 \mathrm{mrad}$ for EMC are combined based on the resolution provided by the different reconstruction techniques. For the EMC analysis, the larger opening angle requirement prevents decay photons from high 

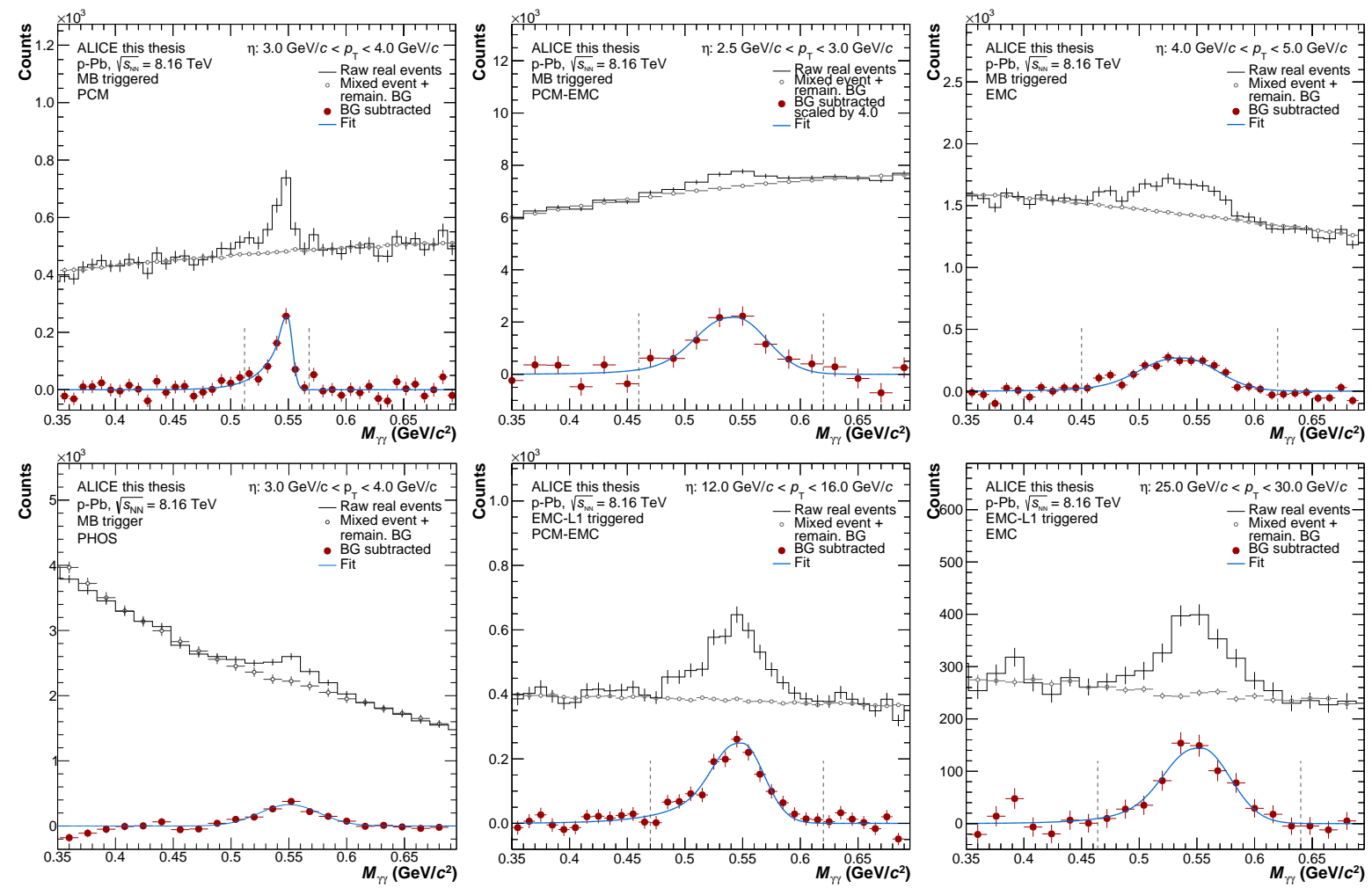

Figure 6.2: Invariant mass distributions in a wide window around the nominal $\eta$ meson mass for $\mathrm{p}-\mathrm{Pb}$ collisions at $\sqrt{s_{\mathrm{NN}}}=8.16 \mathrm{TeV}$ minimum bias data $($ top $)$ and triggered data (bottom) for PCM (left), PCM-EMC (middle), EMC (right) and PHOS (left).

$p_{\mathrm{T}} \pi^{0}$ decays from hitting the EMCal surface in the same cell. This therefore suppresses cluster merging of the photons and in return improves the energy resolution of the reconstructed $\pi^{0}$ meson candidates. Due to the higher mass of the $\eta$ meson, its decay photons are produced at larger opening angles and thus this cut has a negligible effect for the heavier meson. The cut itself is slightly larger than a cell width $(14 \mathrm{mrad})$ in order to deal with the limited projectivity of the EMCal in the $\eta-\varphi$ plane as well as possible primary vertex shifts along the $z$-axis. In addition, the leading cells of the photon candidate clusters are not allowed to be neighboring cells in the EMC method. As seen in the invariant mass distributions, the combinatorial background from the event mixing is not able to fully describe the background under the peak especially for the $\eta$ meson. This is due to further photon correlations in the event, for example from in-jet photons produced in the initial parton shower or from three particle decays (e.g. $\eta \rightarrow \pi^{0} \pi^{0} \pi^{0}$ ). The removal of this background source is absorbed into the combined signal and background fit by assuming that it follows a linear functional shape. The functional form to describe the event mixing subtracted distribution is given by

$$
\begin{array}{r}
y=A \cdot\left\{G\left(M_{\gamma \gamma}\right)+\exp \left(\frac{M_{\gamma \gamma}-M_{\pi^{0}, \eta}}{\lambda}\right)\left[1-G\left(M_{\gamma \gamma}\right)\right] \theta\left(M_{\pi^{0}, \eta}-M_{\gamma \gamma}\right)\right\}+B+C \cdot M_{\gamma \gamma} \\
\text { with } G\left(M_{\gamma \gamma}\right)=\exp \left[-0.5\left(\frac{M_{\gamma \gamma}-M_{\pi^{0}, \eta}}{\sigma_{M_{\gamma \gamma}}}\right)^{2}\right]
\end{array}
$$

where $G$ stands for a Gaussian distribution around the mean value $M_{\pi^{0}, \eta}$ with a width of $\sigma_{M_{\gamma \gamma}}$ and an amplitude given by $A$. The Gaussian is combined with a one-sided exponential tail for $M_{\gamma \gamma}<M_{\pi^{0}, \eta}$ which has an inverse slope given by $\lambda$ and is enabled with a Heavyside function $\theta\left(M_{\pi^{0}, \eta}-M_{\gamma \gamma}\right)$. For the analysis of the EMCal-triggered data Equation 26 is extended by an additional exponential tail term for the $M_{\gamma \gamma}>M_{\pi^{0}, \eta}$ side of the peak to account for 
cluster cluster overlap and resolution effects smearing the reconstructed invariant mass to larger values. The remaining background under the peak is described with the linear function given by $B+C \cdot M_{\gamma \gamma}$ and shown separately from the remaining fit function in Figure 6.1 and Figure 6.2 by the open gray points. The exponential tail in this fit function is necessary to describe the tails on the either side of the invariant mass peak as seen in the example distributions. The left side tail seen for PCM originates from electron bremsstrahlung which results in an energy loss and thus a smearing of the peak to lower invariant masses while for PCM-EMC and EMC late photon conversions are responsible for a cluster energy smearing resulting in lower invariant
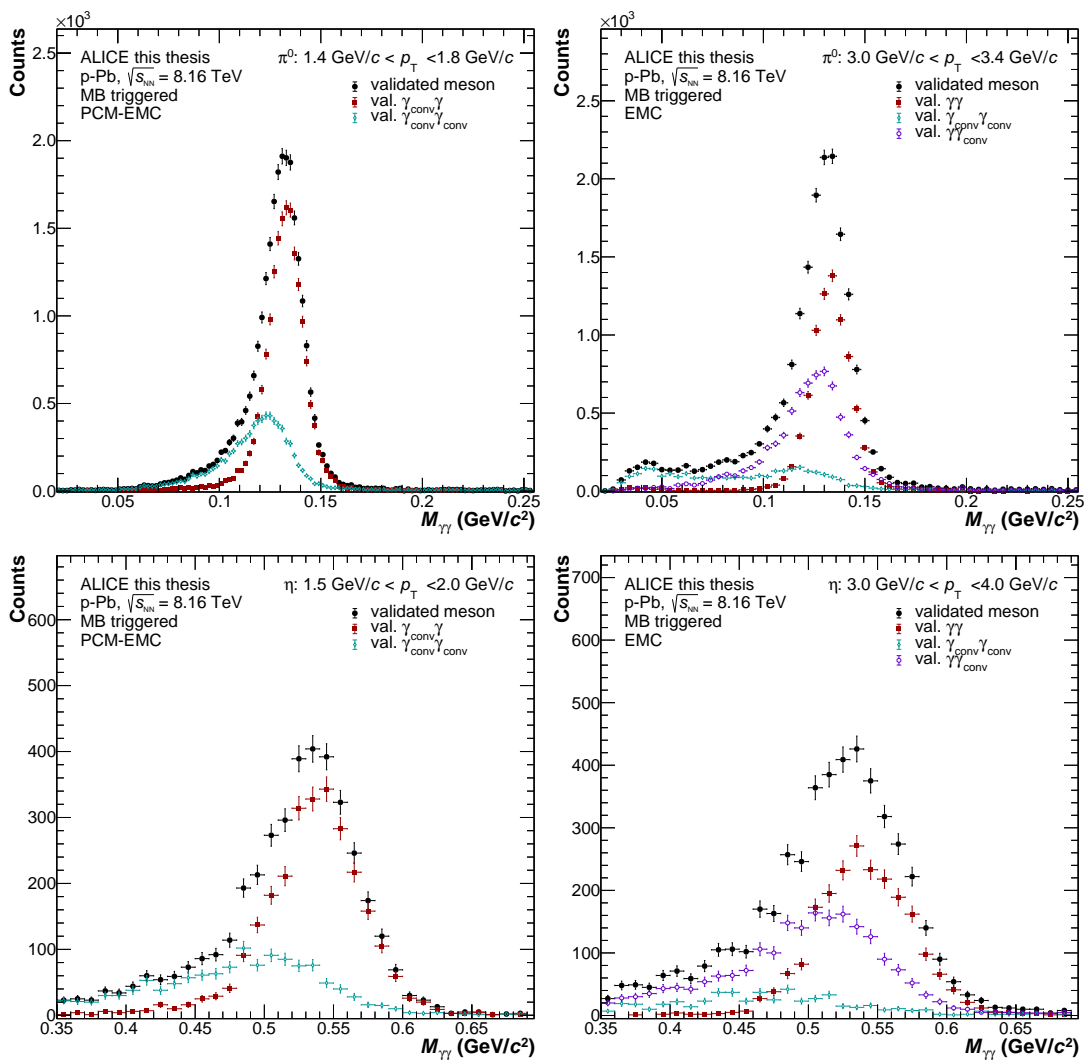

Figure 6.3: True validated invariant mass distributions in a wide window around the nominal $\pi^{0}(t o p)$ and $\eta$ (bottom) meson mass for $\mathrm{p}-\mathrm{Pb}$ collisions at $\sqrt{s_{\mathrm{NN}}}=8.16 \mathrm{TeV}$ DPMJET simulation data shown for PCM-EMC (left) and EMC (right). Contributions from combinations of true photons and late photon conversion clusters are shown separately in different colors.

masses. This smearing of

the distributions is shown in Figure 6.3 where for PCM-EMC (left) and EMC (right) the distributions in DPMJET MC simulations are shown for the various possible combinations of converted photons and true photons. If both clusters for the invariant mass calculation originate from late conversions, the invariant mass is shifted significantly to lower values. The fits according to Equation 26 are used in all $p_{\mathrm{T}}$ bins in order to obtain the peak width as well as the peak position. The peak width is calculated as the full width at half maximum (FWHM) via $2.35 \cdot \sigma_{M_{\gamma \gamma}}$ and the peak positions are given by $M_{\pi^{0}, \eta}$. The latter are in addition used as starting points for the raw yield peak integration. Both values are shown in Figure A.3 for pp and in Figure 6.4 for $\mathrm{p}-\mathrm{Pb}$ for all reconstruction techniques including the external inputs provided by PHOS [196, 203]. As can be seen, the peak positions in data and simulation agree in all methods within the per-mille level due to the extensive efforts that went into the calibration of data and simulation as described in Chapter 5. A similar behavior is observed for the peak widths which show a good agreement between data and simulation while at the same time reflecting the resolution ordering of the different reconstruction techniques. The PCM method exhibits a superior resolution of 2-4 MeV/c $c^{2}$ up to $10 \mathrm{GeV} / c$, while the increasing inclusion of EMCal photons for EMC and PCM-EMC decreases the resolution due to the worse energy resolution of clusters compared to the high tracking resolution of ITS and TPC. Another feature seen in the peak positions is the strong rise of $M_{\gamma \gamma}$ for $p_{\mathrm{T}}>16 \mathrm{GeV} / c$ for EMC $\pi^{0}$ 's, which is a result of shower merging or shower overlaps that are sensitive to the opening angle cut that is applied in the analysis. This cut slowly 

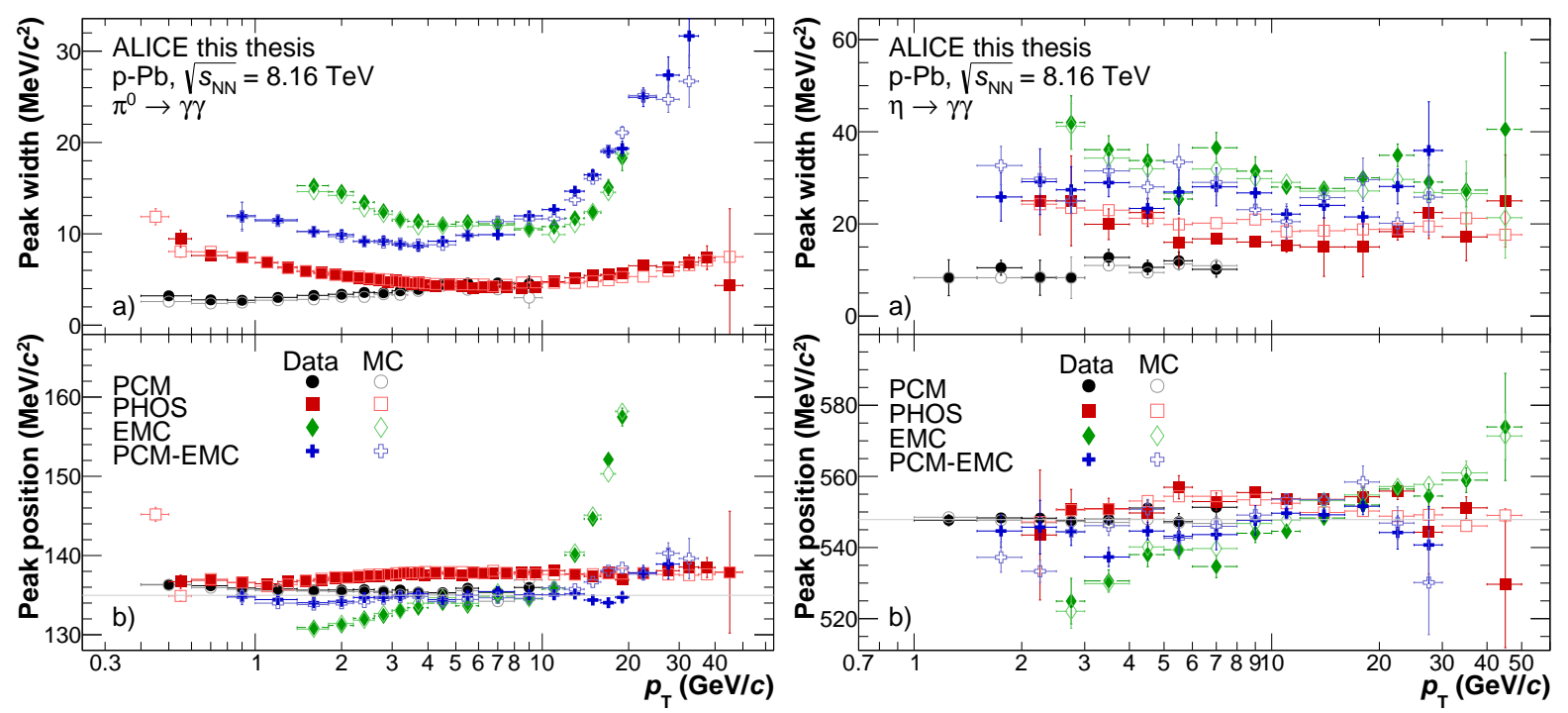

Figure 6.4: Invariant mass peak positions (bottom panels) and width (top panels) obtained from the fits according to Equation 26 for the $\pi^{0}$ (left) and $\eta$ meson (right) versus transverse momentum for $\mathrm{p}-\mathrm{Pb}$ collisions at $\sqrt{s_{\mathrm{NN}}}=8.16 \mathrm{TeV}$. Data is shown in full markers for PCM (black), PCM-EMC (blue), EMC (green) and PHOS (red) while the corresponding MC is shown in open markers.

removes the lower invariant region of the distribution for high $p_{\mathrm{T}}$ thus shifting the peak position to larger values until for $p_{\mathrm{T}}=20 \mathrm{GeV} / c$ the EMC method is no longer able to resolve the $\pi^{0}$ meson decay photons as two separate clusters. For the $\eta$ meson similar observations are made but with the caveat of significantly larger statistical uncertainties due to a reduced production of the heavier meson and a lower two-photon decay branching ratio compared to the $\pi^{0}$ as well as a much larger background under the invariant mass signal peak.

The signal extraction of the neutral meson raw yield is done via integration in terms of bin counting of the background subtracted invariant mass distributions (red points in Figure 6.1 and Figure 6.2) in fixed invariant mass windows around the peak positions obtained from the fits given in Figure 6.4. These integration windows are indicated in the invariant mass distributions with the gray vertical dotted lines. Each reconstruction technique has been assigned a different mass window for integration based on its respective resolution. Moreover, for triggered data the integration windows have been widened due the slightly worsened resolution. The windows are generally asymmetric in order to account for the smearing of the invariant mass distribution to smaller or higher values and a detailed list of all windows is given in Table 6 .

\begin{tabular}{llcc}
\hline Method & Trigger & $\boldsymbol{\pi}^{\mathbf{0}}\left(\mathrm{GeV} / c^{2}\right)$ & $\boldsymbol{\eta}\left(\mathrm{GeV} / c^{2}\right)$ \\
\hline PCM & MB & {$\left[M_{\pi^{0}}-0.030, M_{\pi^{0}}+0.015\right]$} & {$\left[M_{\eta}-0.068, M_{\eta}+0.032\right]$} \\
PCM-EMC & MB & {$\left[M_{\pi^{0}}-0.032, M_{\pi^{0}}+0.022\right]$} & {$\left[M_{\eta}-0.060, M_{\eta}+0.055\right]$} \\
& EMCal L1 (low) & {$\left[M_{\pi^{0}}-0.035, M_{\pi^{0}}+0.029\right]$} & {$\left[M_{\eta}-0.066, M_{\eta}+0.060\right]$} \\
& EMCal L1 (high) & {$\left[M_{\pi^{0}}-0.045, M_{\pi^{0}}+0.037\right]$} & {$\left[M_{\eta}-0.072, M_{\eta}+0.066\right]$} \\
EMC & MB & {$\left[M_{\pi^{0}}-0.050, M_{\pi^{0}}+0.040\right]$} & {$\left[M_{\eta}-0.080, M_{\eta}+0.080\right]$} \\
& EMCal L1 (low) & {$\left[M_{\pi^{0}}-0.050, M_{\pi^{0}}+0.060\right]$} & {$\left[M_{\eta}-0.080, M_{\eta}+0.080\right]$} \\
& EMCal L1 (high) & {$\left[M_{\pi^{0}}-0.060, M_{\pi^{0}}+0.080\right]$} & {$\left[M_{\eta}-0.080, M_{\eta}+0.080\right]$} \\
\hline
\end{tabular}

Table 6: Invariant mass integration windows used for the raw yield integration of the $\pi^{0}$ and $\eta$ meson. $M_{\pi^{0}}$ and $M_{\eta}$ represent the peak positions obtained from the fits according to Equation 26. 


\subsubsection{Correction factors}

The neutral meson raw yield obtained from the integration of the invariant mass distributions need to be corrected for various detector effects as well as for contamination from secondary particles or background. In the following, each correction step is explained and its effect on the spectrum is discussed. First, an out-of-bunch pileup correction needed for the PCM analysis is described followed by a correction for the contamination from secondary neutral pions. Afterwards, acceptance as well as reconstruction efficiency for all reconstruction methods are determined and finally the treatment of the various event triggers is explained.

\subsubsection{Out-of-bunch pileup}

A significant portion of neutral mesons in the PCM raw yield does not stem from the current collision but instead from previous or subsequent bunch crossings due to the long electron drift time and therefore equally long read-out time of the TPC with about 92 $\mu \mathrm{s}$. This is further worsened by the fact that many of the low $p_{\mathrm{T}}$ tracks used in the PCM analysis are reconstructed only with TPC tracking information as the conversions appear in the outer layers of the ITS or in the TPC inner field cage and thus no ITS track points are available to constrain the tracks to the primary vertex. Furthermore, the high luminosities and therefore high interaction rates during the $2012 \mathrm{pp}$ and the $2016 \mathrm{p}-\mathrm{Pb}$ data taking campaigns resulted in an environment that allowed for a large fraction of out-of-bunch collisions to occur during the TPC readout window. For the pp data, the main and satellite bunches in the LHC were brought to collisions, which resulted in small bunch spacing as shown previously in Figure 5.9, thus further increasing the pileup. As there is no information available on the pileup tracks to individually remove them from the analysis, a statistical approach is employed in order to reject the resulting $\pi^{0}$ candidates. This approach is based on the distance of closest approach (DCA) in beam direction $(z)$ of the $\mathrm{V}^{0}$ photon candidates compared to the primary vertex position, called $d c a_{z}$. It is calculated by extrapolating the momentum vector of the $\mathrm{V}^{0}$ candidate to the beam axis and calculating the smallest distance between the primary vertex and this vector. In the pileup determination three different categories of photon candidates are considered based on the tracking detector information on the conversion electron tracks. Both electrons either have no ITS track points available (category 1), one of the electrons has at least two ITS track points (category 2) or both electrons have at least two ITS track points (category 3). The first category of photons where only TPC tracking information is available holds true for all conversions with $R_{\text {conv }}>50 \mathrm{~cm}$ and

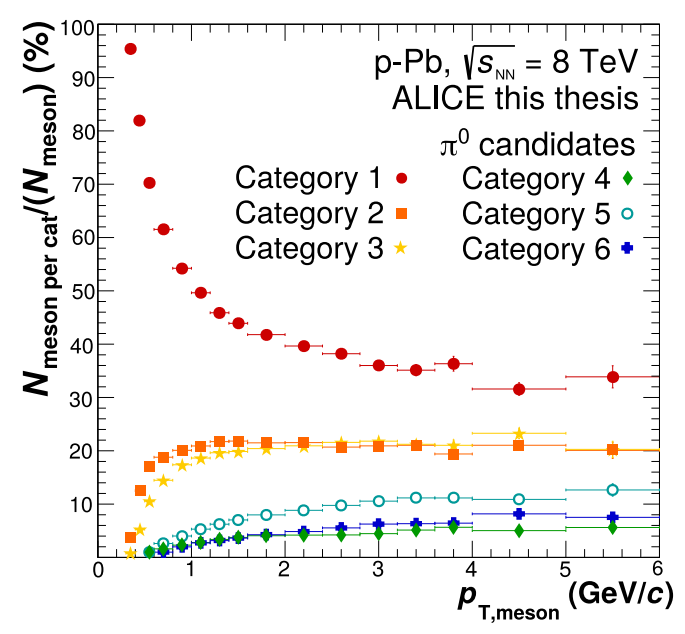

Figure 6.5: Fraction of $\pi^{0}$ candidates in each of the categories based on the track quality of their photon constituents in $\mathrm{p}-\mathrm{Pb} \sqrt{s_{\mathrm{NN}}}=8.16 \mathrm{TeV}$.

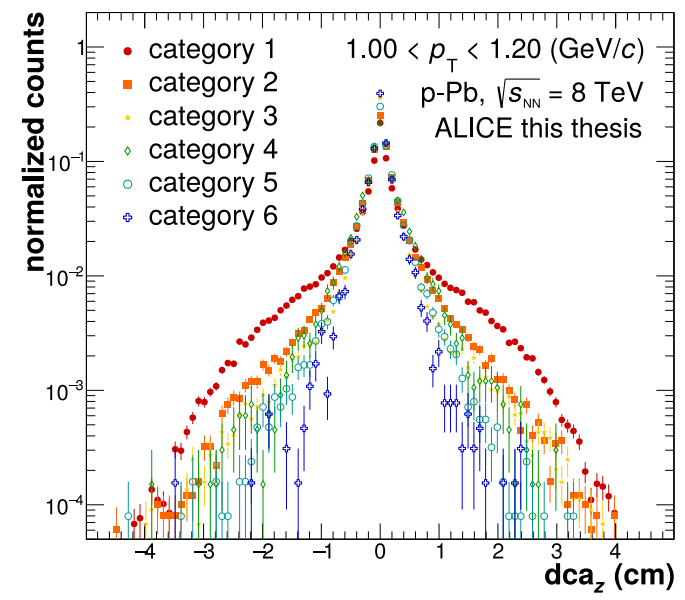

Figure 6.6: $d c a_{z}$ distribution of photons from $\pi^{0}$ candidates for the different categories according to Table 7 in $\mathrm{p}-\mathrm{Pb}$ $\sqrt{s_{\mathrm{NN}}}=8.16 \mathrm{TeV}$. 
affects more than $60 \%$ of the low momentum photon candidates as an electron that could have ITS hits would require at least a transverse momentum of $p_{\mathrm{T}}>120 \mathrm{MeV} / c$ in order to still reach the TPC. Category 2 and 3 are considered to be unaffected by out-of-bunch pileup due to the much better time and position resolution of the ITS which allows to constrain tracks to the primary vertex. Category 2 photons are also less frequent than category 3 photons as one usually

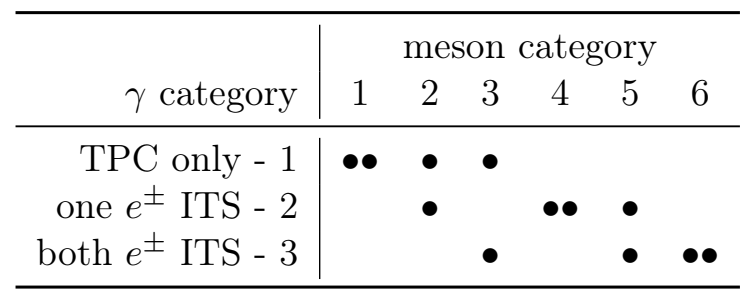

Table 7: Overview of the photon compositions of the different meson categories considered for the out-of-bunch pileup determination. obtains the same amount of ITS clusters on each electron track for early conversions. However, dead areas in the ITS as well as overlapping space points of both electrons can result in reduced ITS information on one leg leading to a category 2 photon. The three photon categories now allow to differentiate between six meson categories with the first category consisting of TPC only tracking and the last category of tracking with at least 2 ITS hits on each of the four electrons. The meson categories from the photon combinations are described in Table 7 where each circle represents one of the two decay photons. The categories are ordered from highest to lowest contamination of out-of-bunch pileup.

The fractions of $\pi^{0}$ candidates in each of the categories is shown in Figure 6.5 for $\mathrm{p}-\mathrm{Pb}$. The high pileup affected category 1 candidates are dominant throughout the $p_{\mathrm{T}}$ range and make up nearly all candidates at low momentum. The out-of-bunch pileup correction factor will therefore have to correct for a much larger fraction at low transverse momentum. Furthermore, for each category the $d c a_{z}$ distribution at low $p_{\mathrm{T}}$ is shown in Figure 6.6 presenting a significantly wider distribution for the TPC only tracking category 1 meson candidates. The widening originates from constraining the out-of-bunch photons to the wrong vertex in the $d c a_{z}$ calculation as well as from a decreased pointing resolution for late conversions in the outer ITS or inner TPC material. This widened distribution due to pile-up is also not present in MC simulations were only single collisions are generated and propagated through the detector as seen in Figure 6.7. The underlying gaussian-like distribution from pileup is then described with varying methods

where the main parametrization is done using the "ShowBackground" function implemented in ROOT. This function follows an iterative procedure to describe a given distribution without its peak and can be modified in terms of smoothness, number of iterations or general expected background shape. It is shown in the example distribution in Figure 6.7 as the blue line which captures the widened part of the $d c a_{z}$ data distribution perfectly. The parameters of the function are optimized so that data and simulation agree on a percent level after subtraction as seen in the subtracted data points (cyan) compared to MC (red points). The background estimation is performed in various $p_{\mathrm{T}}$ bins which are optimized in terms of statistics and can therefore differ in bin width from the $p_{\mathrm{T}}$ binning used for the raw yield extraction. The resulting contamination from out-of-bunch pileup in the $\pi^{0}$ raw yield is show in Figure 6.8 for $\mathrm{p}-\mathrm{Pb}$ versus transverse momentum. A contamination of more than $35 \%$

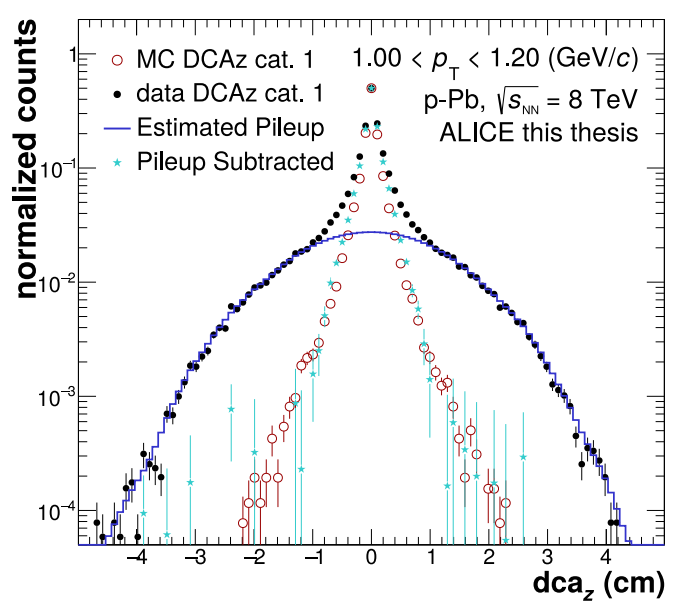

Figure 6.7: $d c a_{z}$ distribution of photons from category $1 \pi^{0}$ candidates in $\mathrm{p}-\mathrm{Pb} \sqrt{s_{\mathrm{NN}}}=8.16 \mathrm{TeV}$ data and MC together with the ShowBackground-based pileup estimate. is observed for $p_{\mathrm{T}}<0.6 \mathrm{GeV} / c$ with values of about 
$15 \%$ at high $p_{\mathrm{T}}$ which is about $8 \%$ more than in pp at high $p_{\mathrm{T}}$. The higher contamination in $\mathrm{p}-\mathrm{Pb}$ stems from the higher interaction rate compared to pp. Due to the slightly different binning in the pileup estimation compared to the raw yield extraction, the contamination is fitted with a power-law function, as shown in Figure 6.8, which is able to describe the distribution over the full $p_{\mathrm{T}}$ range. Based on this function the corresponding fraction of out-of-bunch pileup is evaluated in each $p_{\mathrm{T}}$ slice and subtracted from the meson raw yield.

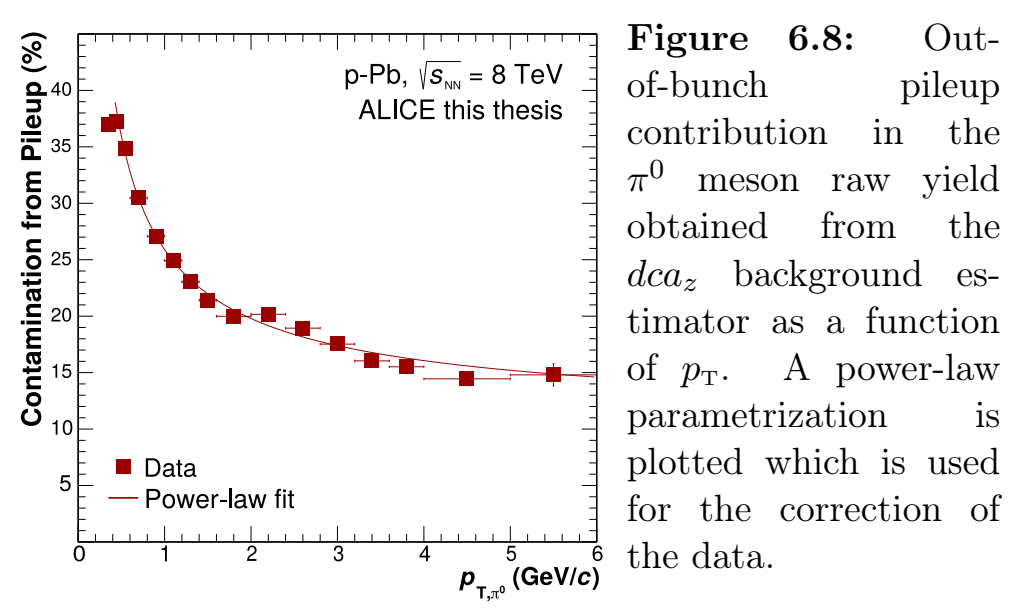

\subsubsection{Secondary neutral pions}

The raw yield obtained from the integration of the invariant mass distributions is not a pure sample of primary neutral mesons. Instead it contains contributions from secondary $\pi^{0}$ mesons from decays of long-lived strange particles, namely $\mathrm{K}_{\mathrm{S}}^{0}, \mathrm{~K}_{\mathrm{L}}^{0}$ and $\Lambda$, as well as contributions from neutral pions produced in hadronic interactions in the material. The latter is obtained from Monte Carlo simulations with full propagation through the ALICE detector via GEANT 3 [173]. For this propagation, a well described implementation of the material and geometry of the ALICE detector is available in the AliRoot framework [164]. The resulting correction exhibits a strong $p_{\mathrm{T}}$ dependence with about 1-2\% correction for PCM for $p_{\mathrm{T}}<0.6 \mathrm{GeV} / c$ while for intermediate $p_{\mathrm{T}}(2-6 \mathrm{GeV} / c)$ the correction factors for PCM and PCM-EMC are only up to $0.2 \%$ whereas for EMC the correction factor is around $0.5-1 \%$ in this region.

Using the same approach for the contributions from weak decays is not feasible as the absolute fractions in simulation and data do not match to a sufficient degree. Instead, a particle decay simulation based on parametrizations of measured or extrapolated spectra is used, which is combined with reconstruction efficiencies and acceptance corrections obtained from Monte Carlo simulation that include a full detector propagation. A detailed overview of the simulation is given in Section 7.1. For pp, no measurements of the required strange particle spectra exist and therefore extrapolations from mea-

\begin{tabular}{llll}
\hline particle & $\begin{array}{l}\text { decay } \\
\text { channel }\end{array}$ & $\begin{array}{l}\text { branching } \\
\text { ratio }\end{array}$ & $\begin{array}{l}\text { decay } \\
\text { length }(c \tau)\end{array}$ \\
\hline$K_{S}^{0}$ & $\pi^{0} \pi^{0}$ & $30.69 \%$ & $2.6844 \mathrm{~cm}$ \\
\hline$K_{L}^{0}$ & $\pi^{0} \pi^{0} \pi^{0}$ & $19.46 \%$ & $15.34 \mathrm{~m}$ \\
& $\pi^{+} \pi^{-} \pi^{0}$ & $12.50 \%$ & \\
\hline$\Lambda$ & $\mathrm{n} \pi^{0}$ & $35.80 \%$ & $7.89 \mathrm{~cm}$ \\
\hline
\end{tabular}

Table 8: Strange particles and a selection of their weak decay channels that include $\pi^{0}$ mesons with the respective branching ratios and decay lengths used in the particle decay simulation [19]. surements in pp collisions at $\sqrt{s}=2.76$ and $7 \mathrm{TeV}[195,206]$ are made. For the $\mathrm{p}-\mathrm{Pb}$ analysis, preliminary measurements of charged particles are available covering the low to intermediate $p_{\mathrm{T}}$ region [207]. In both collision systems, charged kaon measurements are used as proxies for the $\mathrm{K}_{\mathrm{S}}^{0}$ and $\mathrm{K}_{\mathrm{L}}^{0}$ parametrization as their spectra were studied in the past to agree on the percent level and the uncertainties on the charged kaon spectra are significantly smaller. The strongest contribution to the secondary $\pi^{0}$ meson yield is expected to come from the $\mathrm{K}_{\mathrm{S}}^{0}$ decay while the 
$\mathrm{K}_{\mathrm{L}}^{0}$ decay contributions are largely suppressed due to the significantly larger decay length as given in Table 8. The same table also lists the branching ratios and decay channels that are considered for the particle decay simulation. For the particle decay simulations the spectra are parametrized with modified Hagedorn functions given by

$$
\frac{\mathrm{d} N}{\mathrm{~d} y \mathrm{~d} p_{\mathrm{T}}}=p_{\mathrm{T}} \cdot A \cdot\left[\exp \left(a p_{\mathrm{T}}+b p_{\mathrm{T}}^{2}\right)+\frac{p_{\mathrm{T}}}{p_{0}}\right]^{-n},
$$

which provides an excellent description of the spectral shape over the full $p_{\mathrm{T}}$ range. The simulation of particle decays is handled by PYTHIA6.4 [208] and the resulting decay and mother particle spectra are weighed with the input parametrizations to obtain the correct abundances. In order to subtract the secondary $\pi^{0}$ contamination, the spectra from the particle decay simulation need to be converted to raw yield by applying their respective acceptance and efficiency correction factors. These factors for the secondary decay pions differ from those of the primary pions and are therefore separately determined using the full Monte Carlo simulations that include the detector response.

The reconstruction efficiencies for the different secondary $\pi^{0}$ sources are shown in Figure 6.9 for the EMC reconstruction technique. Strong differences are visible especially between the long and short lived neutral kaons, whose decay $\pi^{0}$ are produced either close or far away from the calorimeter, respectively. These $\pi^{0}$, which are produced late and thus at large radii, experience a worse momentum resolution in the EMCal reconstruction, as their production vertex is assumed to be the IP in the EMCal cluster momentum calculation. Similar observations can be made for the other reconstruction techniques where the secondary $\pi^{0}$ efficiencies are systematically smaller than the primary $\pi^{0}$ reconstruction efficiency and differences between the various sources are present. The resulting relative fractions of secondary $\pi^{0}$ mesons in the raw yield are shown in Figure 6.10 for the contributions from the $\mathrm{K}_{\mathrm{S}}^{0}$ for all

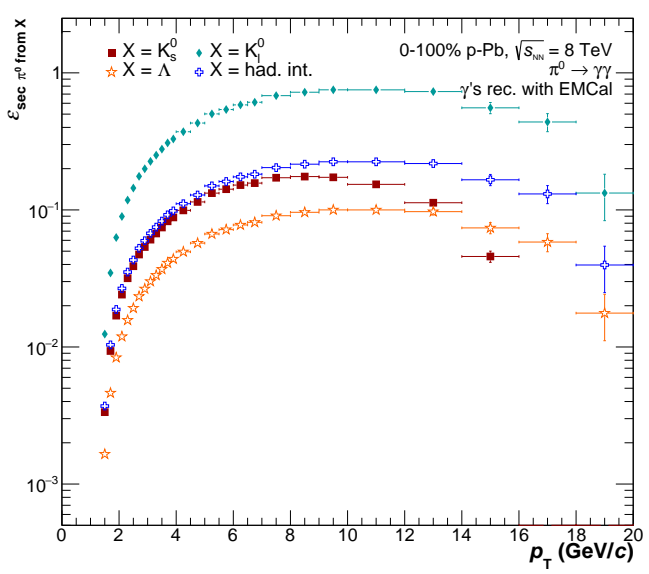

Figure 6.9: Reconstruction efficiency for secondary $\pi^{0}$ mesons from various sources for EMC reconstruction in $\mathrm{p}^{-}$ $\mathrm{Pb}$ collisions at $\sqrt{s_{\mathrm{NN}}}=8.16 \mathrm{TeV}$. reconstruction techniques used in $\mathrm{pp}$ (left) and $\mathrm{p}-\mathrm{Pb}$ (right). The respective fractions for all other sources are given in Figure A.4 for both collision systems. The fractions show a strong $p_{\mathrm{T}}$ and method dependence with the correction for PCM being the lowest with $0.5-2 \%$ and the correction for mEMC being the largest. At low momentum the fractions increase for the methods including PCM photons due to the $\mathrm{V}^{0}$ constrains that force the particles to point to the primary vertex. The contributions from the other particles are nearly negligible compared to the $\mathrm{K}_{\mathrm{S}}^{0}$ with fractions on the order of much less than $1 \%$. The contamination from $\pi^{0}$ mesons from material interactions is on the order of a percent depending on $p_{\mathrm{T}}$. The secondary contributions are then subtracted from the $\pi^{0}$ raw yield for all reconstruction methods before any further correction is applied.

\subsubsection{Kinematic acceptance and reconstruction efficiency}

In order to get from the out-of-bunch pileup and secondary contamination corrected raw yield to fully corrected spectra, two additional corrections are required: the kinematic acceptance correction and the reconstruction efficiency. Both corrections are obtained from the Monte Carlo simulations introduced in Section 4.3, however a special acceptance treatment for the decay photon triggered jet-jet MC simulations is required. The kinematic acceptance is calculated via 
the fraction of $\pi^{0}$ or $\eta$ mesons where both decay photons point onto the respective detector acceptance for the different methods compared to all $\pi^{0}$ or $\eta$ mesons that are generated in the rapidity window $|y|<0.8$. For PCM both decay photons as well as their conversion products have to point into the TPC acceptance with $0<\varphi<2 \pi$ rad and $|\eta|<0.8$ while for PCM-EMC one photon has to follow the PCM acceptance requirement and the other photon has to point on EMCal/DCal. The EMCal acceptance for the pp analysis with the 2012 data is $1.4<\varphi<$ $3.15 \mathrm{rad}$ for $|\eta|<0.67$ while for the $2016 \mathrm{p}-\mathrm{Pb}$ data this is extended to $1.4<\varphi<3.28 \mathrm{rad}$ and an additional $4.56<\varphi<5.7 \mathrm{rad}$ for $|\eta|<0.67$ to account for the DCal modules. For the EMC analysis, both photons have to point into the respective EMCal/DCal acceptance. In the decay photon triggered MC simulations that are used for the correction of the EMCal-triggered data, the acceptance correction contains a strong bias due to the requirement of having a decay photon point to the EMCal surface in each event. As the kinematic acceptance of the $\pi^{0}$ and $\eta$ mesons is based purely on decay kinematics and not on detector effects, this bias can be recovered by using separate generator-level decay simulations to obtain the acceptance correction factor. For this, both neutral mesons are generated individually flat in $p_{\mathrm{T}}$ and in the full azimuth for a large rapidity window of $|y|<1.2$ using PYTHIA8.2 and the resulting decay information is used to calculate the kinematic acceptance correction factors based on the respective EMC and PCM-EMC detector acceptance.

The reconstruction efficiency is calculated solely using the full detector MC simulations by performing the reconstruction procedure the same way as it is done for data. It is then calculated via the fraction of reconstructed $\pi^{0}$ or $\eta$ meson candidates with respect to all generated mesons that pass the acceptance criteria. Furthermore, a second type of efficiency, the "true" reconstruction efficiency is determined by validating on the reconstructed sample which candidates consist of two true photons that come from the same primary meson. Both efficiencies agree on a percent level depending on the invariant mass background description quality, which is worst for EMC due to a visible shape difference at low invariant masses.

Figure 6.11 shows the normalized correction factor $\epsilon$ that contains the reconstruction efficiency $\epsilon_{\text {rec }}$, the acceptance $A$ and the purity $P$ which is only needed for mEMC as described in Section 6.2. The factor reflects the overall ability of each reconstruction technique to reconstruct neutral mesons depending on their transverse momentum. It is strongly affected by the selection criteria used in the analysis as well as the reconstruction technique itself which employs different detector systems. For PCM it shows the strong effect of the conversion probability of about $9 \%$ for a single photon which enters twice for the neutral meson reconstruction while for EMC
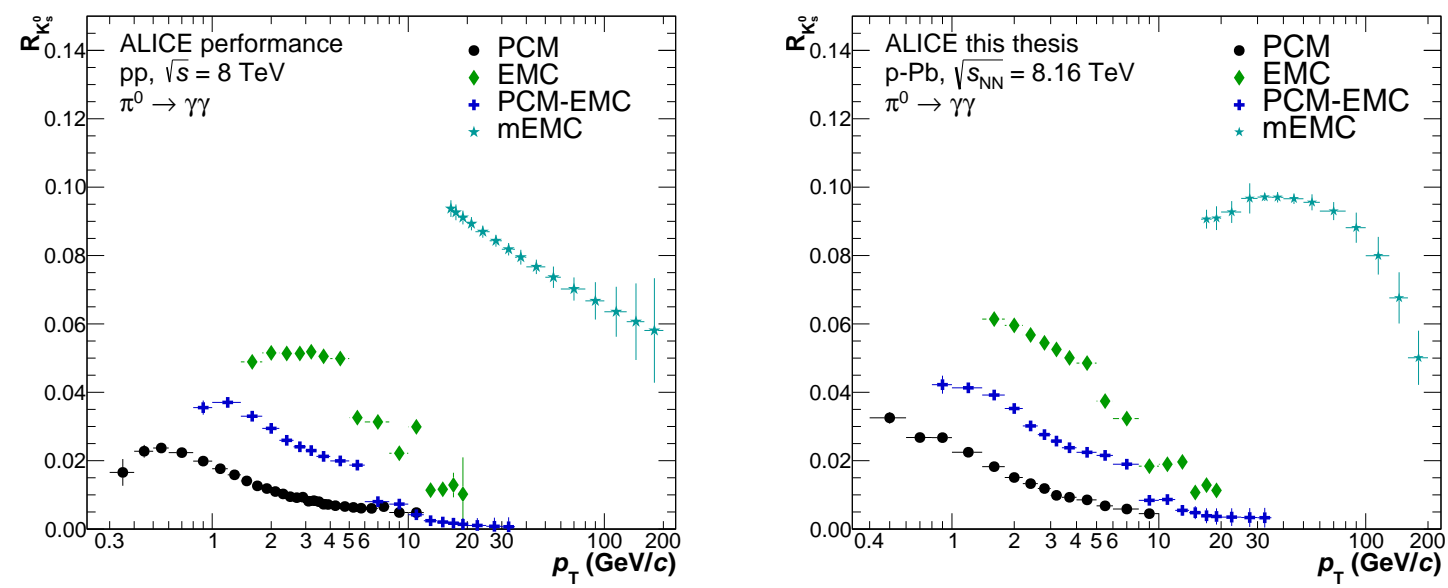

Figure 6.10: Effective correction factors for $\pi^{0}$ mesons from $K_{S}^{0}$ decays for the different reconstruction techniques used in $\mathrm{pp}$ (left) and in $\mathrm{p}-\mathrm{Pb}$ (right). 

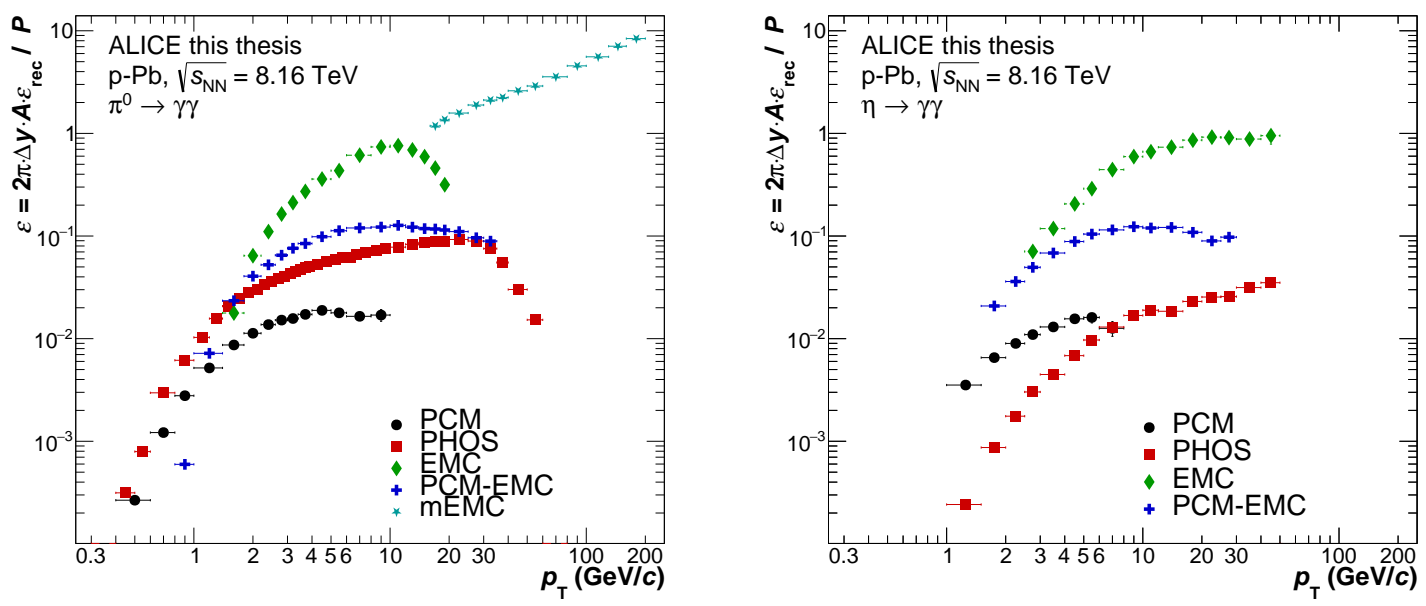

Figure 6.11: Reconstruction efficiency for $\pi^{0}$ (left) and $\eta$ (right) mesons from the different reconstruction techniques in $\mathrm{p}-\mathrm{Pb}$ collisions at $\sqrt{s_{\mathrm{NN}}}=8.16 \mathrm{TeV}$ normalized by the detector acceptances and including the purity correction for mEMC.

the minimum cluster energy cut causes a vanishing efficiency at low momentum. The kinematic acceptance has very minimal effect on the $\pi^{0}$ mesons for PCM for $p_{\mathrm{T}}>1 \mathrm{GeV} / c$, however for the $\eta$ mesons a strong $p_{\mathrm{T}}$ dependence is observed due to the much larger opening angle resulting from the higher meson mass. Especially for PHOS the $\eta$ acceptance is small due to the detector geometry. With the acceptance for both mesons reaching similar values in all reconstruction techniques (except PHOS) at high $p_{\mathrm{T}} \approx 10 \mathrm{GeV} / c, \epsilon$ approaches the same values for $\pi^{0}$ and $\eta$ at these momenta. The total correction factor $\epsilon$ is smallest for PCM reaching at most $1-2 \%$ while for EMC it approaches values of $80 \%$ and PCM-EMC is located in-between with values around $10 \%$ in the plateau region. Furthermore, for EMC only symmetric decays are possible at very low momentum while additional phase space opens towards higher momentum. However, above $10 \mathrm{GeV} / c$ the $\pi^{0}$ decay photon clusters in the EMCal start to merge which one tries to counter with a minimum opening angle cut in the analysis. Therefore, the efficiency starts to drop again towards higher momenta which is not observed for the $\eta$ meson where merging appears at much higher $p_{\mathrm{T}}$ which are not reached in the presented analysis.

\subsubsection{EMCal triggered data}

For the EMC and PCM-EMC reconstruction techniques, EMCal triggered data is used to enhance the statistics as well as the high $p_{\mathrm{T}}$ reach of the spectra. One effect of the triggers is a reduction in reconstruction efficiency below the trigger threshold. The onset and width of this effect depends on the trigger threshold of the respective EMCal trigger and has been simulated using the trigger maker framework as described in Section 4.3.1. The bias on the efficiency is described by $\kappa_{\text {Trig }}$ which is the ratio of the efficiencies with and without the trigger maker emulation. It is shown in Figure 6.12 for PCM-EMC and EMC in $\mathrm{p}-\mathrm{Pb}$ for the EG2 and EG1 triggers for the $\pi^{0}$ reconstruction efficiency. $\kappa_{\text {Trig }}$ is steeply falling towards low $p_{\mathrm{T}}$ and approaches unity for high $p_{\mathrm{T}}$ as expected. The trigger turn-on shows a different width depending

on the reconstruction technique due to a smearing for PCM-EMC from the different decay

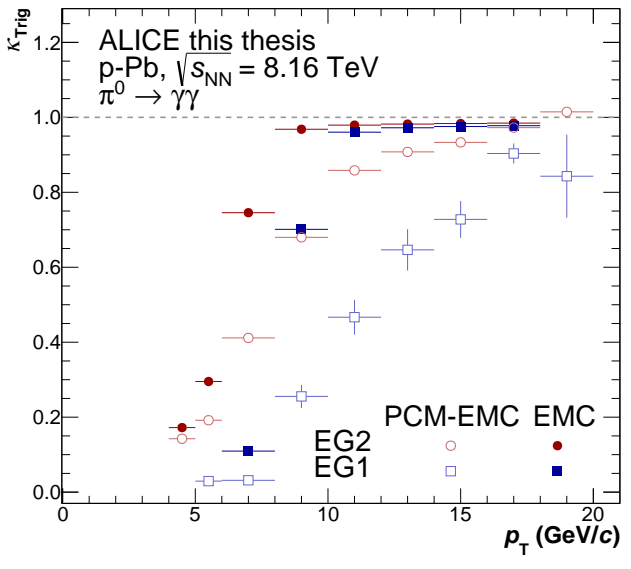

Figure 6.12: Trigger efficiency bias for the EMCal Level 1 triggers EG2 and EG1 for $\pi^{0}$ mesons reconstructed with PCM-EMC (open markers) and EMC (full markers). 

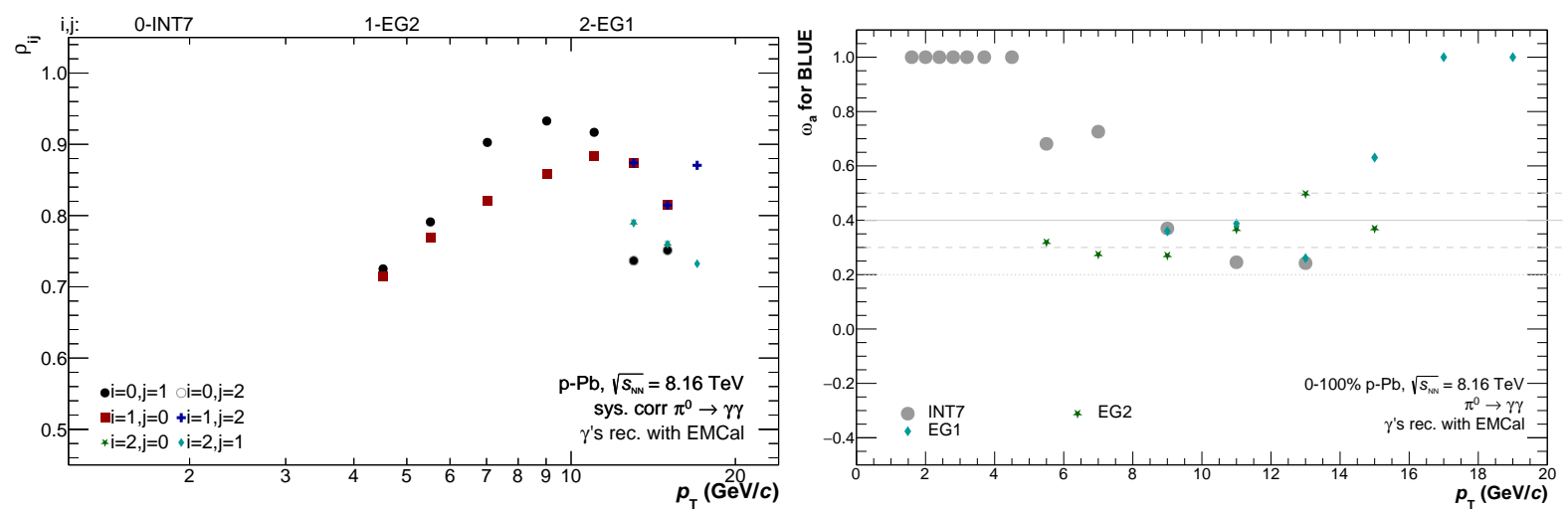

Figure 6.13: Left: Correlation factors $\rho_{i j}$ of the $\pi^{0}$ meson spectra between the minimum bias and the EMCal L1 triggers. Right: Weights $\omega_{a}$ for the BLUE method combination of the $\pi^{0}$ meson spectra reconstructed with EMC in the different triggered samples.

kinematics as well as the reduced chance to trigger on the conversion electrons. With the trigger emulation and the resulting correction for the trigger efficiency bias, the EMCal triggered data can be corrected in the same way as the minimum bias data. This results in separate spectra for each triggered data sample. In total, three triggers are used in the pp and the $\mathrm{p}-\mathrm{Pb}$ analyses for the measurement of the neutral meson spectra. Based on the various spectra, one fully combined spectrum for each of the reconstruction techniques is obtained using the Best Linear Unbiased Estimate (BLUE) method $[209,210]$. The combination procedure creates a weighted average defined by

$$
\begin{aligned}
\left\langle Y\left(p_{\mathrm{T}}\right)\right\rangle & =\omega^{T}\left(p_{\mathrm{T}}\right) Y\left(p_{\mathrm{T}}\right) \\
& =\sum_{a=1}^{n} \omega_{a}\left(p_{\mathrm{T}}\right) Y_{a}\left(p_{\mathrm{T}}\right),
\end{aligned}
$$

where $n=3$ is the number of available individual measurements that should be combined. The individual $p_{\mathrm{T}}$-dependent spectra are denoted as $Y_{a}\left(p_{\mathrm{T}}\right)$ which are assigned the weights $\omega_{a}$. Furthermore, each spectrum enters the combination procedure with its own set of statistical, systematic and resulting total uncertainties which can be denoted as $R_{i}\left(p_{\mathrm{T}}\right), S_{a}\left(p_{\mathrm{T}}\right)$ and $T_{a}\left(p_{\mathrm{T}}\right)$, respectively. A $6 \times 6$ matrix $(C)$, as given by

$$
C_{i j}\left(p_{\mathrm{T}}\right)=\frac{\rho_{i j} S_{i}\left(p_{\mathrm{T}}\right) \rho_{j i} S_{j}\left(p_{\mathrm{T}}\right)}{T_{i}\left(p_{\mathrm{T}}\right) T_{j}\left(p_{\mathrm{T}}\right)}
$$

is used to describe the full correlations with individual coefficients correlating a given trigger $i$ with another trigger $j$. Here, $\rho_{i j}$ denotes the $p_{\mathrm{T}}$-dependent fractional amount of correlations in the systematic uncertainties between trigger $i$ and $j$. The $p_{\mathrm{T}}$-dependence of these fractions is shown in Figure 6.13 (left) exemplary for the EMC $\pi^{0}$ spectra in $\mathrm{p}-\mathrm{Pb}$ for the minimum bias trigger INT7 and the two EMCal L1 triggers EG2 and EG1. The $\rho_{i j}$ fractions present values of $55-96 \%$ as correlations between the different triggers where $100 \%$ would correspond to fully correlated measurements. The yield extraction uncertainties in this case are assumed to be fully uncorrelated and remaining uncertainties are correlated to a strong degree. This strong correlation is a result of the analysis selection criteria having similar effects on the clusters or $\mathrm{V}^{0}$ candidates in the various trigger samples. The criteria mainly depend on differences in the detector conditions, like between various periods, than on different event triggers that are analyzed within the same data set. The $p_{\mathrm{T}}$-dependent weights $\omega_{a}\left(p_{\mathrm{T}}\right)$ for Equation 29 are then 
given by

$$
\omega_{a}\left(p_{\mathrm{T}}\right)=\frac{\sum_{b=1}^{n} C^{-1}}{\sum_{a, b=1}^{n} C^{-1}},
$$

based on the correlation matrix elements. They are shown exemplary for the different triggers used in the EMC based $\pi^{0}$ measurement in $\mathrm{p}-\mathrm{Pb} \sqrt{s_{\mathrm{NN}}}=$ 8.16 TeV in Figure 6.13 (right) and similar plots for the other reconstruction techniques can be found in the appendix in Section A.5. Those figures also show the $p_{\mathrm{T}}$ range in which the triggered data is considered for the combination. For both EMCal triggers, the data is only used slightly above the trigger threshold as the trigger maker emulation does not provide a perfect efficiency description around the trigger turn on. The comparison of the individual $\pi^{0}$ meson spectra from the different triggers to a combined fit is shown in Figure 6.14 for the EMC method in $\mathrm{p}-\mathrm{Pb}$. In general, the triggered spectra agree with each other as well as with the minimum bias spectrum within $1-10 \%$ which is well within the statistical or total uncertainties. The resulting spectra from the combination procedure then profit from the statistics of the individual samples as well as the reduction of systematic uncertainties due to correlations between the measurements. Furthermore, this procedure allows to cover a much larger transverse momentum range from very low $p_{\mathrm{T}}$ using the minimum bias triggered data up to the highest measurable $p_{\mathrm{T}}$ within the given statistics of the highest EMCal L1 trigger.

\subsection{Reconstruction (mEMC)}

With the invariant mass based techniques a statistical limit is reached above a certain transverse momentum in the presented data. In addition, the reconstruction methods themselves reach a limit at high $p_{\mathrm{T}}$, when the two decay photons of the $\pi^{0}$ meson (or of the $\eta$ for even higher $p_{\mathrm{T}}$ ) can no longer be resolved separately in the detectors due to their decreasing opening angle. In order to extend the $\pi^{0}$ measurements to momenta beyond the invariant mass limit, a different type of analysis is employed: the merged cluster analysis. In this thesis the method is employed for EMCal, but it could theoretically also be performed using the PHOS where merging appears at much higher momenta due to its finer granularity. The basic principle of this method is that the $\pi^{0}$ decay photons at high momentum are produced under such small opening angles that the resulting electromagnetic showers in the EMCal can not be resolved separately and instead overlap or merge. This merging is shown in Figure 6.15 for the V2 clusterizer which is used in the analysis. The fig-
Figure 6.14: Comparison of the $\pi^{0}$ meson spectra reconstructed with EMC with three different event triggers (INT7, EMCal-EG2, EMCal-EG1) to a combined fit in $\mathrm{p}-\mathrm{Pb}$ data at $\sqrt{s_{\mathrm{NN}}}=8.16 \mathrm{TeV}$.

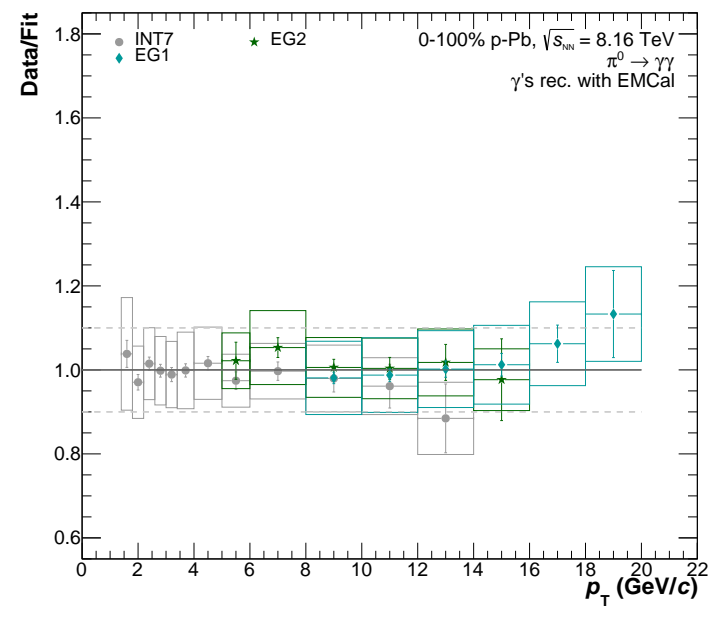

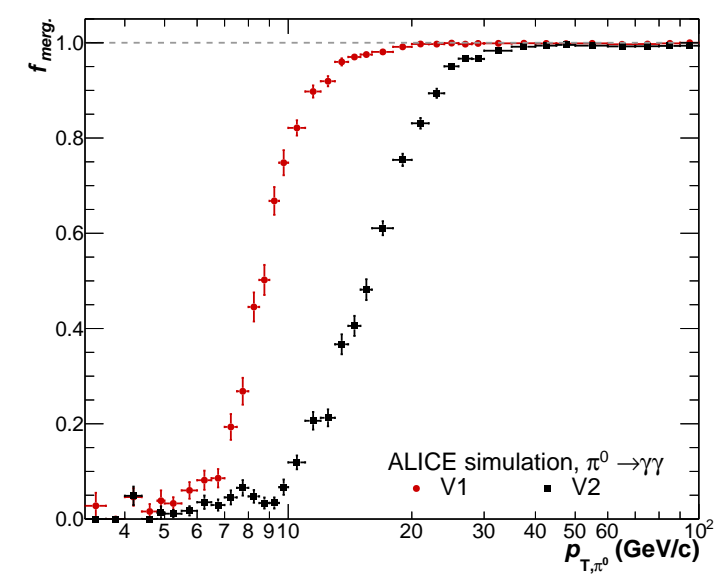

Figure 6.15: Fraction of $\pi^{0}$ mesons where the decay photons can not be resolved as separate clusters in the EMCal, based on full detector Monte Carlo simulations with two clusterizers [150]. 
ure shows that starting from $p_{\mathrm{T}} \approx 10 \mathrm{GeV} / c$ decay photons, depending on the asymmetry of the decay, are contained within the same cluster. For $p_{\mathrm{T}}>16 \mathrm{GeV} / c$ already close to half of all decay photon pairs can no longer be resolved separately which strongly affects the invariant mass based methods and is the reason for the enforced opening angle cut in the EMC analysis. The cluster merging in EMCal is in addition illustrated in Figure 6.16 which compares clusters measured within the EMCal granularity to a calorimeter with near infinite resolution. At high momentum (right panels) both photons are too close to each other in order to deposit the main fraction of their energy in separate cells and thus the V2 clusterizer can no longer split the cluster (indicated by the red ellipse which includes the cells of the cluster found by the V2 clusterizer). In contrast, the left panel shows this for decay photons from an intermediate $p_{\mathrm{T}} \pi^{0}$ where splitting is possible and is indicated by the red line. The merged cluster analysis itself follows

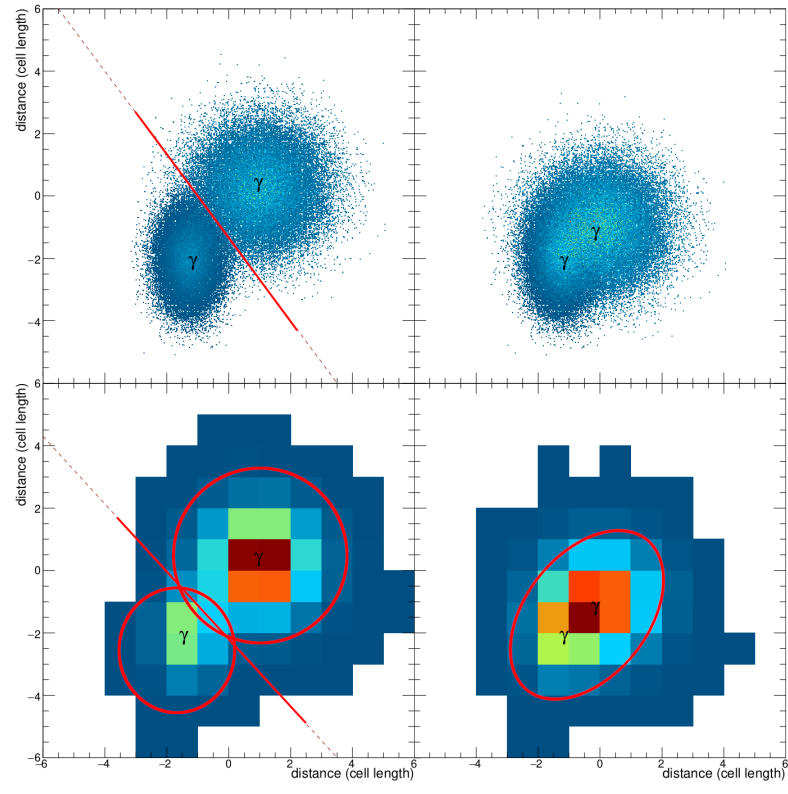

Figure 6.16: Comparison of photon shower resolution in an infinite granularity calorimeter $(t o p)$ and the ALICE EMCal (top). The approximate cluster splitting from the V2 algorithm is indicated by a red line.

a particle identification approach similar to measurements of charged particles in ALICE. In this approach, basic analysis cuts provide a high purity in the $\pi^{0}$ sample and remaining corrections rely strongly on the correct description of the cut quantities as well as on the particle composition in the simulations. One of the main discriminators to identify merged clusters is the shower shape of the cluster described by $\sigma_{\text {long }}^{2}$ as given in Equation 20. Merged clusters exhibit a more elliptic shower distribution in the $\eta-\varphi$ plane compared to single photon clusters due to the overlap of the two photon clusters which is reflected by larger values of $\sigma_{\text {long }}^{2}$. It is therefore crucial for the $\mathrm{MC}$ simulations to agree with data in the shape of the shower shape variable distributions which was achieved by the cross-talk emulation as described in Section 5.2.4. Unfortunately, further distinctions between single photon and merged clusters are not possible and the remaining analysis requirements are the same as for a single photon analysis. The very good agreement between data and MC in the shower shape variable $\sigma_{\text {long }}^{2}$ is shown in Figure 6.17 (left) for a high $p_{\mathrm{T}}$ bin of EMCal-EG1 triggered data together with the distribution from PYTHIA8 Jet-Jet simulations
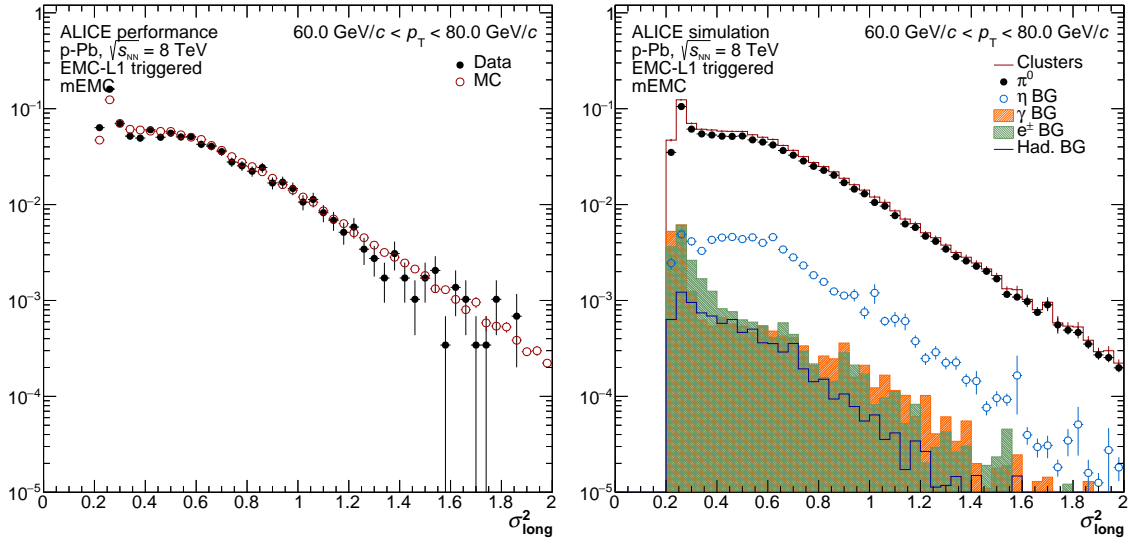

Figure 6.17: Long axis of the shower shape $\sigma_{\text {long }}^{2}$ distributions in data and MC (left) for $\pi^{0}$ meson candidates with $60<p_{\mathrm{T}}<80$ $\mathrm{GeV} / c$. The $\pi^{0}$ signal and background distributions from Jet-Jet MC simulations (right) are given in the same $p_{\mathrm{T}}$ interval. 
in $\mathrm{p}-\mathrm{Pb}$. Small differences are seen around the photon peak located at $\sigma_{\text {long }}^{2} \approx 0.25$ due to missing prompt photon contributions in the Jet-Jet MC simulation which are corrected for later on A very important component of the merged cluster analysis is the effect of photon conversions and the contribution of single photon clusters in the $\pi^{0}$ candidate sample, which directly affects the energy resolution of the reconstructed candidates. As seen in Figure 5.1 in Section 5 a significant portion of material is located in front of the EMCal due to the TRD and the TOF detectors with about $0.3-0.4 X / X_{0}$ in terms of radiation length. For high energetic photons, both conversion electrons of such late conversions can be fully contained in the same cluster due to the large boost of the particles. However, it is also possible that only a single electron of the $\pi^{0}$ candidate is contained in the cluster. As it is not possible on analysis level to distinguish the cluster candidates of pure merged clusters from those containing contributions from photon conversions, four different types of $\pi^{0}$ candidates are considered in the analysis: (a) fully merged clusters where both photons or two of the three Dalitz decay particles are contained in the cluster, $(b)$ merged clusters where at least one photon has converted and a minimum of one conversion electron from the second photon is contained in the cluster, (c) "merged" clusters that contain only one of the two $\pi^{0}$ decay photons and $(d)$ "merged" clusters that contain only one electron from a photon conversion. These four candidate classes only differ in their energy resolution of the $\pi^{0}$ candidate but can otherwise show similar shower shapes.

The fraction of each of the classes in the $\pi^{0}$ candidate sample is shown in Figure 6.18 for all clusters with $\sigma_{\text {long }}^{2}>0.27$ which excludes the photon peak region. It shows that the pure merged clusters which contain both photons are not the dominant contribution and instead the category $b$ is dominant where at least one of the photons has converted. In a previous analysis in pp collisions at $\sqrt{s}=2.76 \mathrm{TeV}$, the fraction of type $a$ clusters with respect to the combined type $b$ $d$ categories was much higher due to missing TRD modules in front of the EMCal supermodules [88]. This shows how significant the detectors in front of the EMCal affect the photon conversion probability.

The specific momentum resolution of the different types of merged $\pi^{0}$ candidates is shown in Figure 6.19 which reflects directly the overall loss of energy due

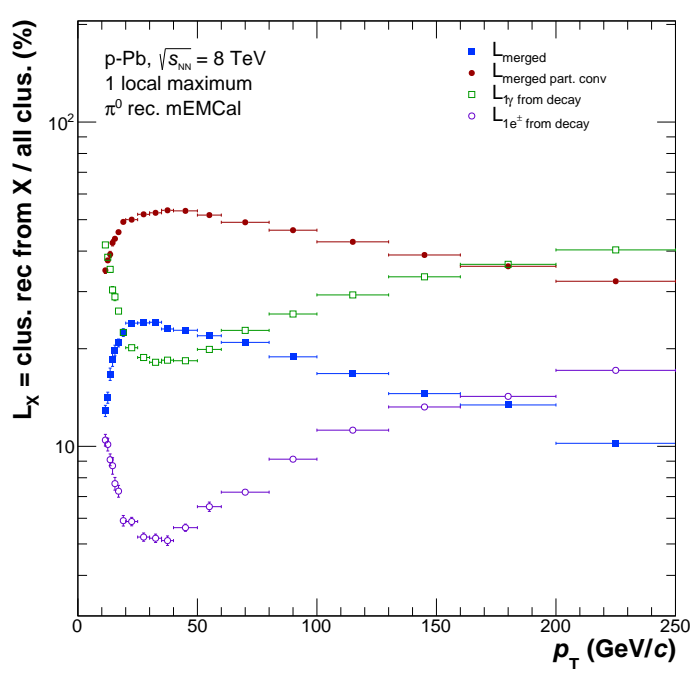

Figure 6.18: Fraction of merged $\pi^{0}$ candidate clusters with $\sigma_{\text {long }}^{2}>0.27$ that contain different contributions of the decay and conversion products.

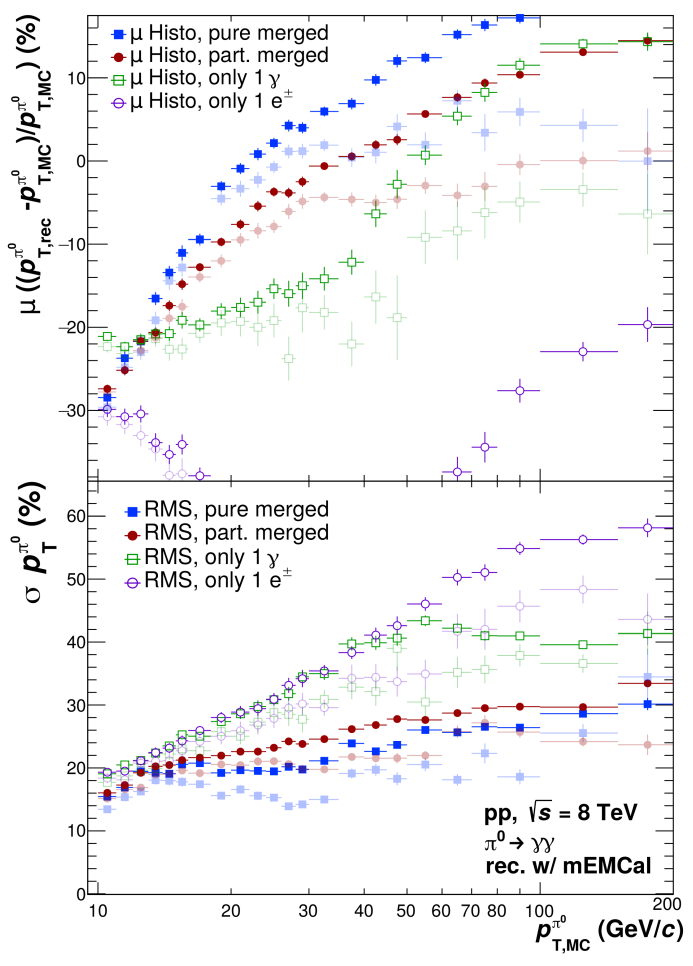

Figure 6.19: Reconstructed versus true momentum shift (top) and momentum resolution (bottom) of $\pi^{0}$ candidates of the four different cluster types in pp. Light markers represent neutralized clusters via track matching while for the dark markers no matching was performed. 
to not capturing all decay and conversion products. The dark markers show the situation without the track matching procedure as described in Section 5.2.2 while in the light markers the neutralization of the clusters is applied. The distributions show that significant portions of the $\pi^{0}$ energy are not part of the cluster for $p_{\mathrm{T}}<20 \mathrm{GeV} / c$. This means that either larger opening angles or a bending of the electron-positron pairs in the magnetic field lead to a splitting of the cluster, while some energy still remains in the main cluster from these split contributions. This explains why, for the pure merged case, strong shifts in the reconstructed versus true momentum are observed. Due to the decay kinematics at $p_{\mathrm{T}}>20 \mathrm{GeV} / c$, more than $90 \%$ of clusters contain both photons. This results in a near vanishing (values around 0) shift of the momentum in the track matched cluster case (light blue points). Without track matching, additional contributions from charged particles inside the jet start to overlap with the main cluster, resulting in momentum shifts to higher values for the cluster types $a$ to $c$. The lower panel presents the root mean square (rms) of the resolution matrix slices. It shows that the resolution for the non-pure merged cases is significantly worse compared to the pure merged case especially towards very high $p_{\mathrm{T}}$. As none of these four cluster types can be separated in data, the corrections for the momentum shift fully rely on the MC simulations and their description of the composition from the different types compared to data. This correction is included in the efficiency calculation which by construction contains the ratio of reconstructed momentum to true momentum.

\subsubsection{Corrections}

For the mEMC analyses, correction factors for acceptance, reconstruction efficiency and purity need to be applied which are based to a large extend on the Jet-Jet MC simulations with full detector propagation as listed in Section 4.3. The acceptance and reconstruction efficiency are calculated similar to the invariant-mass-based methods, but with an additional inclusion of the Dalitz decay channel of $\pi^{0} \rightarrow \gamma e^{+} e^{-}$in the signal definition. For the acceptance calculation only one of the $\pi^{0}$ daughters is required to point to the EMCal in the nominator due to the inclusion of all four cluster types as described in the previous section. In addition, the mEMC analysis limits the rapidity range to $|y|<0.6$ as a reconstruction outside of this window is not possible given the EMCal detector geometry. The resulting acceptance correction factor is flat in $p_{\mathrm{T}}$ with a value of $A_{\pi^{0}} \approx 0.47$. The reconstruction efficiency, calculated according to

$$
\epsilon_{\text {eff }}=\frac{N_{\pi^{0}}^{\text {rec }}\left(p_{\mathrm{T}, \text { rec }}\right)}{N_{\pi^{0},|y|<0.6}^{\text {incaca }}\left(p_{\text {T,true }}\right)}
$$

corrects in addition for the momentum shift of the $\pi^{0}$ candidates as described in the previous section. This resolution correction can also be performed with an unfolding procedure. However, this approach was not taken as the bin widths in the analysis do not guarantee a stable enough unfolding and can therefore introduce biases or large uncertainties. Possible mismatches between data and MC are caught in the systematic uncertainty evaluation described in Section 6.3. Due to the inclusion of all four $\pi^{0}$ candidate types, it is furthermore possible to reconstruct a neutral pion in multiple clusters. For $\pi^{0}$ candidates with $p_{\mathrm{T}}>20 \mathrm{GeV} / c$ this double counting effect accounts for $2.7 \%$ of all candidates without cluster neutralization and for $1.8 \%$ of candidates with track matching and is included in the corrections obtained from MC information.

The reconstructed $\pi^{0}$ candidate sample contains background from various sources. The reconstructed yield therefore needs to be corrected for the $p_{\mathrm{T}}$-dependent purity $(P)$, which is given by

$$
P\left(p_{\mathrm{T}}\right)=\frac{N_{\mathrm{val}, \mathrm{rec}}^{\pi^{0}}\left(p_{\mathrm{T}}\right)}{N_{\mathrm{rec}}^{\pi^{0}}\left(p_{\mathrm{T}}\right)}=1-\sum_{i=1}^{n} c_{i}\left(p_{\mathrm{T}}\right) .
$$


It represents the fraction of reconstructed and validated $\pi^{0}$ candidates $\left(N_{\text {val,rec }}^{\pi^{0}}\right)$ to all reconstructed candidates $\left(N_{\text {rec }}^{\pi^{0}}\right)$. It can alternatively be expressed via a summation of all relative contamination sources $\left(c_{i}\right)$ in the sample. The fractions $c_{i}$, obtained from the Jet-Jet MC simulations, are shown in Figure 6.20 and are dominated by the $\eta$ meson contamination with about $10 \%$ at high $p_{\mathrm{T}}$ followed by percent-level contamination from electrons and fragmentation photons. At low $p_{\mathrm{T}}$, charged pion contamination contributes with 1-2\%. However, without the use of the track matching procedure, as done in the $\mathrm{p}-\mathrm{Pb}$ analysis, it becomes dominant in this region with more than $10 \%$. In both cases, the charged pion fraction becomes negligible for high $p_{\mathrm{T}}$ similar to contamination from other hadronic background sources like $\mathrm{K}_{\mathrm{L}}^{0}$, neutrons and protons which are only on the per-mille level. For the dominant contributions, additional corrections are applied in order to correct for improper abundances in the Jet-Jet MC simulations. For the $\eta$ meson contamination, this is done by comparing the high $p_{\mathrm{T}}$ values of the $\eta / \pi^{0}$ ratio in data and $\mathrm{MC}$ and correcting the $c_{\eta}$ fraction by the resulting difference. Under the assumption that $m_{\mathrm{T}}$-scaling holds true up to $p_{\mathrm{T}} \approx 200 \mathrm{GeV} / c$, the difference in constant fits on the high $p_{\mathrm{T}}$ regions of the $\eta / \pi^{0}$ ratios results in corrected values of $c_{\eta} \approx 12 \%$ at high $p_{\mathrm{T}}$. The comparison of the measured $\eta / \pi^{0}$ ratio with the Pyтнia8 Jet-Jet MC prediction is shown in the results chapter in Figure 6.30 together with the constant fit. Moreover, the Jet-Jet MC simulations do not contain contributions from prompt photons (e.g. from $q g \rightarrow q \gamma$ and $q \bar{q} \rightarrow g \gamma$ processes) in the direct photon sample. Instead only partonic $2 \rightarrow 2$ processes are included. This is corrected for by using Pythia8 generator-level simulations where the additional prompt photon producing processes are enabled.

The fractions of prompt and fragmentation photons

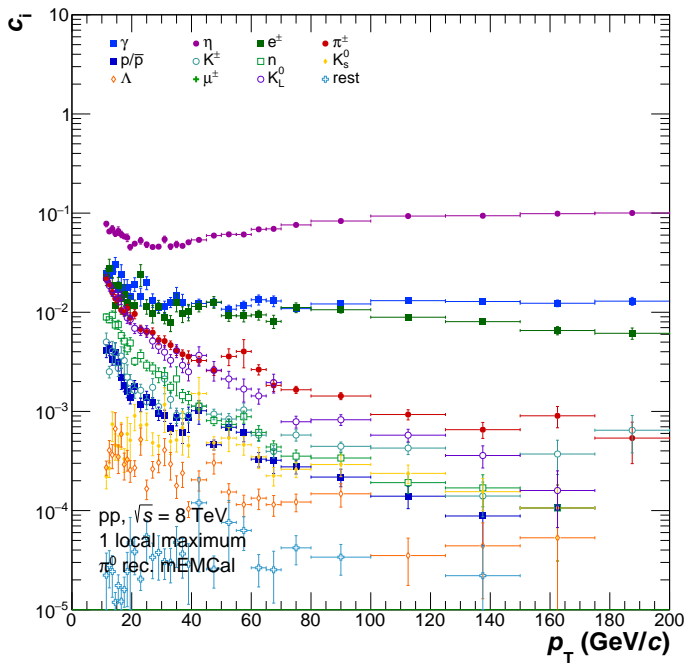

Figure 6.20: Fractional contaminations $c_{i}$ from various particles in the $\pi^{0}$ candidate sample based on Jet-Jet MC simulations.

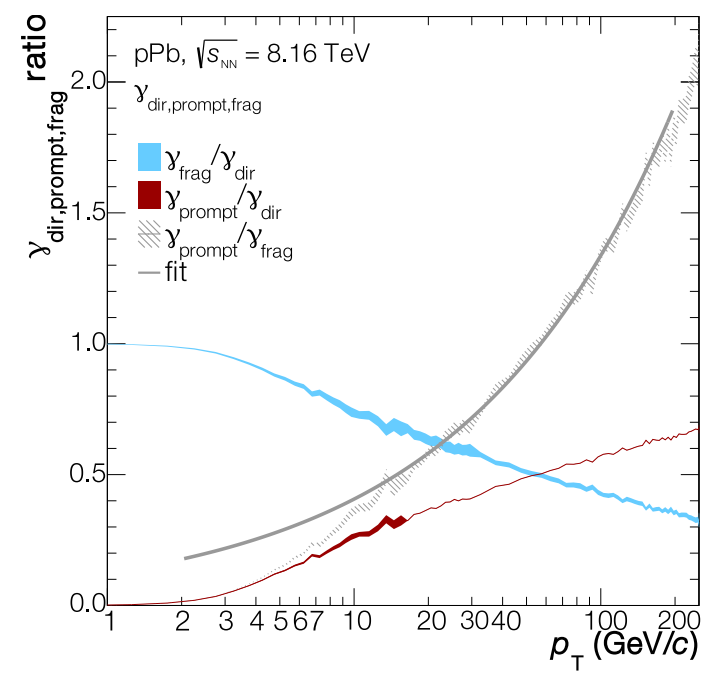

Figure 6.21: Fractions of prompt and fragmentation photons in the direct photon sample together with the $\gamma_{\text {prompt }} / \gamma_{\text {frag }}$ ratio and its functional parametrization. in the direct photon sample as well as the ratio of prompt to fragmentation photons are shown in Figure 6.21. The latter is described with the ratio of two powerlaw functions accounting for the individual photon spectra. This functional form is then used to correct for the missing prompt photons via

$$
\gamma_{\text {frag }+ \text { prompt }}=\gamma_{\text {frag }} \cdot\left[1+\left(\gamma_{\text {prompt }} / \gamma_{\text {frag }}\right)_{\text {fit }}^{\text {Pythia }}\right]
$$

This results in an up to twice as large direct photon contribution at very high $p_{\mathrm{T}}$. Another missing contamination stems from electrons from weak boson $(\mathrm{W}, \mathrm{Z})$ decays which is not included in the Jet-Jet MC simulations. Therefore a correction based on PowhEG + Pythia 8 calculations on 

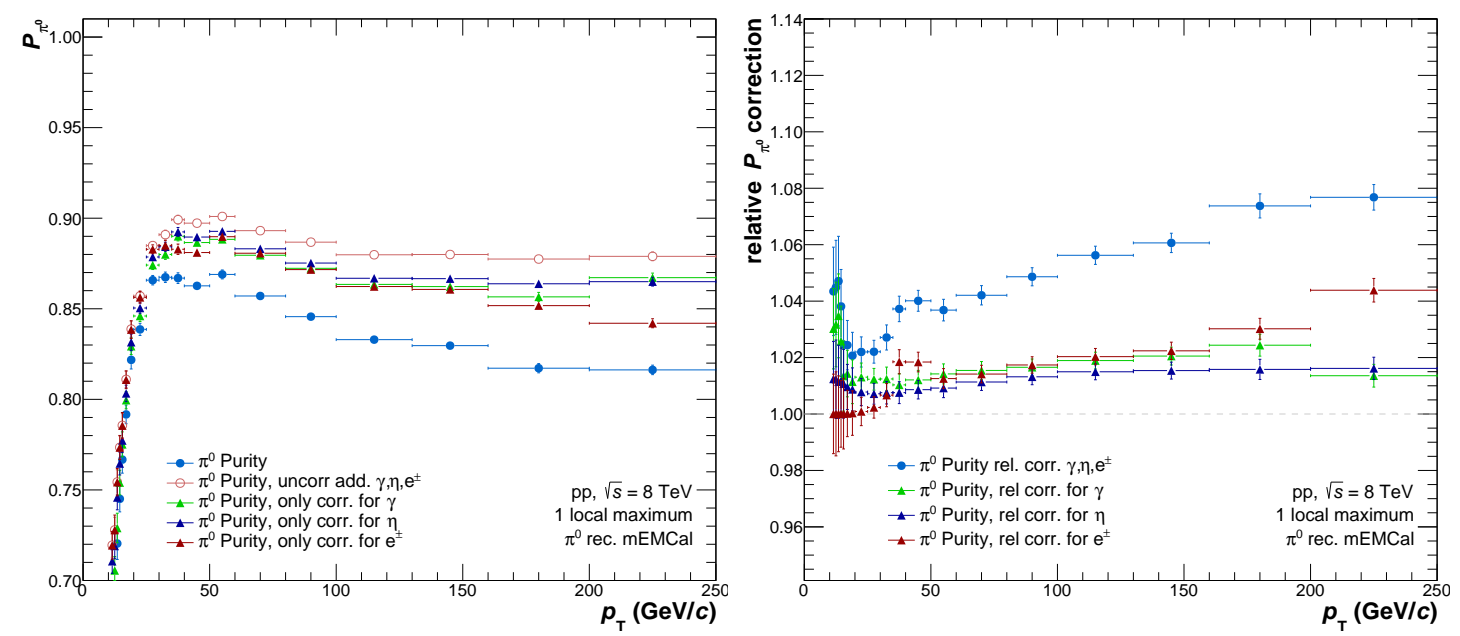

Figure 6.22: Purity of the $\pi^{0}$ candidates reconstructed with mEMC (left) with corrections for contributions of $\gamma_{\text {prompt }}, \eta$ and electrons from weak decays. The relative purity change of each correction is shown relative to the uncorrected purity (right).

top of the electrons from Jet-Jet processes is applied following

$$
e_{\mathrm{JJ}+\text { weak }}=e_{\mathrm{JJ}} \cdot\left[1+\left(e_{\mathrm{weak}} / e_{\mathrm{JJ}}\right)\right] .
$$

Powheg-Box/W and Powheg-Box/Z [211] interfaced with the parton shower of Pythia8 have been used to estimate the additional electrons at NLO level. The ratio of electrons from weak decays to the JJ MC electron sample is unity around $p_{\mathrm{T}}=40 \mathrm{GeV} / c$ and rises up to a factor three at high $p_{\mathrm{T}}$. Taking all contributions into account, the final purity correction factor is shown in Figure 6.22 (left) in blue with the effect of the additional corrections on the $\eta$ meson, $\gamma_{\text {prompt }}$ and electrons from weak decays indicated in different colors. Furthermore, the right panel shows the relative purity change that these corrections introduce with $1-2 \%$ change coming from the $\eta$ abundance correction, 1-3\% from the missing prompt photon and 1-4\% from the missing electrons from weak decays. The final purity of the $\pi^{0}$ candidates in the mEMC analysis is maximal $87 \%$ for $p_{\mathrm{T}} \approx 30-50 \mathrm{GeV} / c$ and decreasing towards high $p_{\mathrm{T}}$ to values of about $82 \%$.

Similar to the invariant mass based methods, secondary neutral pions from $K_{\mathrm{S}}^{0}, K_{\mathrm{L}}^{0}, \Lambda$ as well as from material interactions are subtracted from the mEMC $\pi^{0}$ spectra. This correction is analog to the one described in Section 6.1.1.2 with the material interaction contribution being determined from full detector MC simulations and the remaining contributions from particle decay simulations based on parametrizations of measured spectra. The corrections show a slight $p_{\mathrm{T}}$ dependence with the contribution from $K_{\mathrm{S}}^{0}$ and the material contribution each being $4-7 \%$ while the rest is negligible in the per-mille level. 


\subsection{Systematic uncertainties}

For all reconstruction techniques (PCM, PCM-EMC, EMC and mEMC) a detailed estimation of systematic uncertainties is performed. These uncertainties arise predominantly from the photon and meson selection criteria which are applied on distributions on quantities that are not perfectly reproduced in Monte Carlo simulations as in data. The enforced selection criteria therefore result in up to several percent different candidate samples in data and $\mathrm{MC}$ which affects raw yield and correction factors. The systematic uncertainties are thus determined from individual variations of each selection criterium in order to determine the resulting change on the spectrum. For the $\eta / \pi^{0}$ ratio and the nuclear modification factors, the uncertainties were determined directly on the ratio in order to correctly treat correlations between the uncertainties of the different measurements. An overview of the systematic uncertainties for example $p_{\mathrm{T}}$ bins is given in the appendix in Table 15 to Table 19 for the $\pi^{0}$ and $\eta$ spectra, the $\eta / \pi^{0}$ ratio and the $\pi^{0}$ and $\eta$ nuclear modification factors. For the PHOS measurement that is also considered for comparisons and combination, the systematic uncertainties were determined in Ref. [204] and are not further discussed here.

Cluster description: This uncertainty accounts for the differences between data and simulation regarding the clusterization process as well as the shower shape within the cluster. For this, the minimum cluster energy has been varied from $0.6-0.8 \mathrm{GeV}$ resulting in up to $10 \%$ systematic uncertainty at very low meson $p_{\mathrm{T}}$ and a negligible impact for $p_{\mathrm{T}}>3 \mathrm{GeV} / c$ on both meson spectra. Furthermore, the minimum number of cells in the cluster is varied between one and three resulting in a percent level difference on the spectra for EMC and half the difference for PCM-EMC. Both of these sources are negligible for mEMC as the analysis only considers clusters with $E_{\mathrm{cls}}>16 \mathrm{GeV}$ which is far above the minimum cluster energy cut and such clusters always contain more than 3 cells. The variation on the shower shape $\sigma_{\text {long }}^{2}$ contains for EMC and PCM-EMC a different maximum cut of $\sigma_{\text {long }}^{2}<0.3$ as well as no maximum cut. For mEMC the minimum cut is varied within $\sigma_{\text {long }}^{2}>0.2-0.35$. This results in differences on the spectra of $3-6 \%$ for EMC and 2-5\% for PCM-EMC and mEMC with increasing values towards higher $p_{\mathrm{T}}$. For $\mathrm{mEMC}$ an additional variation is performed on the distance of the cluster maximum to the next bad EMCal channel with either 1 cell width or 1 cell diagonal difference. This uncertainty is considered, as the proximity to bad channels affects the energy resolution and the shower shape calculation of clusters due to missing parts of the shower. The variations amount to a $1 \%$ uncertainty for $p_{\mathrm{T}}<100 \mathrm{GeV} / c$ and increase to about $5 \%$ for higher $p_{\mathrm{T}}$. This variation was made to ensure a proper description of the shower shape by excluding clusters close to bad channels, whose energy does not enter the $\sigma_{\text {long }}^{2}$ calculation. For all methods, variations of the cell timing cut resulted in negligible differences which could be attributed to the comparably large bunch spacings combined with the past-future protection (as explained in Section 4.2) that is applied beforehand. On the $\eta / \pi^{0}$, the uncertainties cancel to a large extend, as the initial cluster sample for both mesons is the same. The uncertainties from this category only partially cancel on the nuclear modification factors, as the material in front of EMCal, as well as the detector geometry differs between the $\mathrm{pp}$ and $\mathrm{p}-\mathrm{Pb}$ measurements. The summed systematic uncertainties on $R_{\mathrm{pA}}$ from the cluster description category amounts to $1-5 \%$, increasing with $p_{\mathrm{T}}$.

Cluster energy nonlinearity and fine tuning: The testbeam nonlinearity correction comes with four systematic variations that were determined within the uncertainties of the PS and SPS data points as seen in Section 5.2.3. For each of these variations, a separate set of cluster energy fine tuning corrections based on the $\pi^{0}$ invariant mass peak positions is determined and the resulting effect on the meson spectra is compared to the default correction. This results in a $2 \%$ systematic uncertainty for EMC and mEMC and 1.4\% for PCM-EMC. In addition, the residual 
difference between data and $\mathrm{MC}$ in the $\pi^{0}$ invariant mass peaks is determined and taking into account the spectral slope which is falling with about $p_{\mathrm{T}}^{-6}$, the resulting energy scale uncertainty is determined to be $1-2 \%$ depending on the reconstruction technique. On the $\eta / \pi^{0}$ ratio, the uncertainties from this category cancel to a combined value of approximately $1 \%$ independent of $p_{\mathrm{T}}$. This is due to a slight difference in the $\pi^{0}$ and $\eta$ Monte Carlo agreement after calibration. On the nuclear modification factor, a summed $2 \%$ contribution remains after cancellations, which is attributed to the residual energy scale uncertainties in both systems.

Cluster neutralization (track matching): The matching of tracks to clusters requires a track propagation to the radial position of the cluster. This propagation as well as the $E / p$ ratio that is used as a matching veto show slight differences between data and MC. Therefore, the matching parameters in $\Delta \eta$ and $\Delta \varphi$ are varied from $p_{\mathrm{T}}$ dependent to fixed values ranging between 0.008 and 0.02 , respectively. In addition, the $E / p$ veto is separately changed from no veto up to $E / p<2$. All variations show only small systematic changes for the invariant mass based analyses of EMC and PCM-EMC of about 1\%. For mEMC, no track matching was available in the EMCal-triggered $\mathrm{p}-\mathrm{Pb}$ data set and thus the uncertainty was determined solely on $\mathrm{pp}$. The uncertainty would largely cancel on $R_{\mathrm{pA}}$, but due to the lack of track matching in the p- $\mathrm{Pb}$ analysis, it has been fully propagated.

$\mathbf{V}^{0}$ track reconstruction: The track quality selection criteria necessary to obtain good tracks for the $\mathrm{V}^{0}$ reconstruction are associated with multiple systematic uncertainty sources. The minimum track transverse momentum cut has been varied between 0.04 and $0.1 \mathrm{GeV} / c$ while the minimum requirement of TPC clusters relative to the number of findable clusters has been probed for values between $35 \%$ and $70 \%$. With those variations, the central barrel tracking with ITS and TPC is tested for systematic differences between data and MC in terms of dead areas, track matching efficiency between TPC and ITS as well as the TPC gas and gain properties. In total, this category of systematic uncertainty sources contributes between 0.4 and $1 \%$ for the $\pi^{0}$ and between $1 \%$ and $2.5 \%$ for the $\eta$ meson for PCM while for PCM-EMC approximately $0.5-1 \%$ larger uncertainties are determined due to the limited sampling of the ITS and TPC region in front of EMCal and DCal. On the $\eta / \pi^{0}$ ratio and the nuclear modification factor, the systematic uncertainties from this category cancel to a large extend and are negligible with less than $0.5 \%$.

Electron identification: The systematic uncertainty assigned to the electron identification as well as the rejection of charged pion contamination via the TPC $\mathrm{d} E / \mathrm{d} x$ energy loss signal is determined via variations of the accepted $\mathrm{n} \sigma$ region as well as the minimum and maximum momentum for the $\pi^{ \pm}$rejection interval. The $\mathrm{n} \sigma$ window around the electron line has been varied to tighter and looser values covering up to $5 \sigma$ while for the pion rejection variations between $0 \sigma$ and $2 \sigma$ at varying $p_{\mathrm{T}}$ intervals were tested. Due to a non-perfect description of the energy loss signal in data compared to MC, with generally more narrow bands for the different species, these variations introduce an approximate $1 \%$ systematic uncertainty on the $\pi^{0}$ spectrum and up to $3 \%$ for the $\eta$ meson. A momentum dependence of the systematic uncertainty with larger values for larger $p_{\mathrm{T}}$ is observed which roughly aligns with the merging of the electron and pion energy loss signals in the TPC $\mathrm{d} E / \mathrm{d} x$ distribution. The uncertainties cancel mostly on the $\eta / \pi^{0}$ ratio and nuclear modification factor, but retain contributions due to different TPC conditions in pp compared to $\mathrm{p}-\mathrm{Pb}$.

PCM photon identification: The systematic uncertainty associated with the photon quality selection criteria is one of the dominant sources for the PCM and PCM-EMC reconstruction techniques. It covers the dominant background rejection criteria on $\chi^{2}$ and $\left|\Psi_{\text {pair }}\right|$ as well as 
the momentum dependent and two-dimensional cuts on the Armenteros-Podolanski distribution. The associated uncertainty from variations to tighter and looser selection criteria exhibits a strong $p_{\mathrm{T}}$ dependence similar to the electron identification uncertainty with values between 2.6 and $6.0 \%$ for the $\pi^{0}$ with PCM and $2.9-7.0 \%$ for PCM-EMC with slighly larger uncertainties on the $\eta$ meson spectrum due to the different decay kinematics of the heavier meson. Due to a difference in signal to background in pp versus $\mathrm{p}-\mathrm{Pb}$ and for the $\pi^{0}$ relative to the $\eta$ meson, the systematic uncertainty sources form this category experience little cancellations in the $R_{\mathrm{pA}}$ and the $\eta / \pi^{0}$ ratio.

Signal extraction: The invariant mass distributions between data and MC show subtle differences due to the underlying background distribution as well as due to the limited detector simulation performance for effects like energy loss. This uncertainty source there contains variations of the invariant mass peak integration windows from the default values given in Table 6 as well as the treatment of the underlying background. For PCM and PCM-EMC these variations result in a small systematic uncertainty of about $1 \%$ at low to intermediate $p_{\mathrm{T}}$ for the $\pi^{0}$ due to the high resolution of the peak which is very well reproduced in simulation. For higher momenta, the uncertainty increases to approximately $5 \%$ due to fitting and signal integration fluctuations in the yield extraction process as well as small systematic differences in the peak positions between data and MC. For EMC, the systematic uncertainty is ranging between $1 \%$ and $9 \%$ with larger uncertainties at very low and very high $p_{\mathrm{T}}$ due to the energy resolution and the onset of cluster merging, respectively. For the $\eta$ meson, generally larger uncertainties are present due to the large width of the peak combined with the strong combinatorial background which is difficult to describe. The uncertainties are therefore roughly double of what they are for the $\pi^{0}$ with very large uncertainties at low $p_{\mathrm{T}}$ where the signal to background ratio is worst. The signal extraction systematic uncertainties are fully propagated to the $\eta / \pi^{0}$ ratio and the nuclear modification factors, making them a dominant source for all invariant mass-based reconstruction techniques.

Secondary contamination: The parametrizations used in the particle decay simulation for the correction of secondary $\pi^{0}$ from weak decays are only constrained up to $p_{\mathrm{T}} \approx 5 \mathrm{GeV} / c$. This is a well enough constrain for the corrections for PCM, PCM-EMC and EMC where the correction factor is on the order of $1-2 \%$ and thus a systematic overestimation or underestimation of the parametrization would not lead to a significant change of the correction factor. The higher $p_{\mathrm{T}}$ region, especially important for $\mathrm{mEMC}$, depends strongly on an extrapolation of the particle spectra parametrizations up to $p_{\mathrm{T}} \approx 250 \mathrm{GeV} / c$, which are assumed to follow a power-law functional form. In addition, the total correction factor in the mEMC method for the secondary $\pi^{0}$ contamination is rather large with up to $6 \%$. Combined with the lack of a high $p_{\mathrm{T}}$ constraint of the spectra, a conservative uncertainty of $4 \%$ on the spectrum is estimated. This uncertainty can only be reduced if new spectra for charged or neutral kaons are made available with a higher $p_{\mathrm{T}}$ reach. On the $\eta / \pi^{0}$ ratio as well as the nuclear modification factors, the secondary contamination uncertainty cancels to a large extend and is thus considered negligible.

Inner and outer material: The largest systematic uncertainty for conversion photon measurements stems from the knowledge of the inner detector material $(5<R<180 \mathrm{~cm})$ which is directly correlated with the conversion probability. There have been in-depth studies of the material in pp collision data at $\sqrt{s}=7 \mathrm{TeV}[193,212,213]$ which determined a conservative uncertainty estimate of $4.5 \%$ independently of transverse momentum. As this uncertainty is assigned per conversion photon, the total systematic uncertainty for PCM on the $\pi^{0}$ and $\eta$ meson reconstruction is $9 \%$ and for PCM-EMC it is $4.5 \%$ while the uncertainty fully cancels in the 

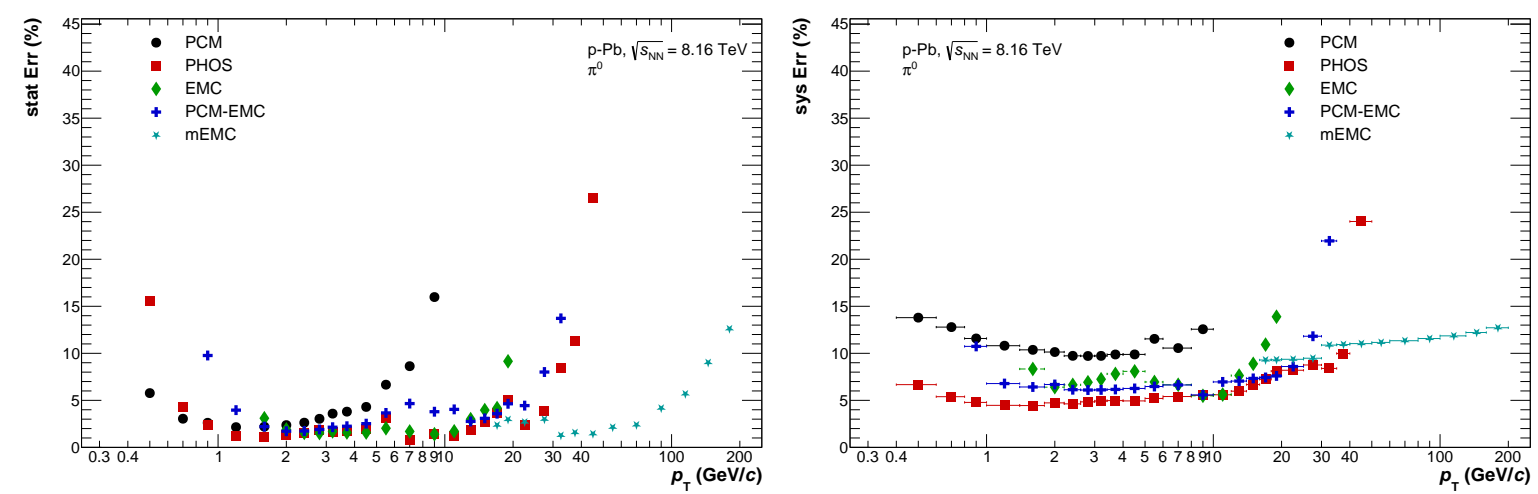

Figure 6.23: Relative statistical (left) and systematic (right) uncertainties on the $\pi^{0}$ meson spectra in $\mathrm{p}-\mathrm{Pb}$ collisions at $\sqrt{s_{\mathrm{NN}}}=8.16 \mathrm{TeV}$. The uncertainties are shown for the different reconstruction techniques and versus transverse momentum.

respective $\eta / \pi^{0}$ ratios and nuclear modification factors. For the EMC-based measurements, an additional uncertainty arises from the outer material budget that is located directly in front of EMCal (and DCal). This material is comprised of the outer TPC wall together with the TRD and the TOF as well as their respective support structures. These detector systems amount to a large combined radiation length of $X / X_{0} \approx 33 \%$ for TRD and TOF which is more than twice the inner detector material. The uncertainty from this material is contained in the amount of conversions it generates and the resulting decrease of the energy resolution for photon detection. In order to determine the uncertainty, pp collision data from 2012 at $\sqrt{s}=8 \mathrm{TeV}$ was used where not all TRD modules were yet installed and thus the TRD only partially covered the EMCal [196]. With this, the $\pi^{0}$ spectrum could be measured separately for supermodules with and without TRD modules in front which resulted in a $4.2 \%$ uncertainty independent of $p_{\mathrm{T}}$. For this, the same material uncertainty for TRD and TOF was assumed and each detector is considered to independent of each other. This uncertainty also fully cancels for the $\eta / \pi^{0}$ ratio and the nuclear modification factor.

Out-of-bunch pile-up: The out-of-bunch pileup correction used in the PCM analysis highly depends on the quality of the ShowBackground description of the $d c a_{z}$ distribution. As this form of pileup is not simulated in $\mathrm{MC}$, an uncertainty for the parametrization is determined by varying the ShowBackground parameters for the background shape as well as for the magnitude, which is largely affected by the amount of iterations used in the procedure. These variations result in $3-5 \%$ and $1-4 \%$ systematic uncertainty for the shape and iteration parameters, respectively, with larger values at low $p_{\mathrm{T}}$ due to the increasing amount of TPC-only tracks in this region. This uncertainty is fully propagated to the $\eta / \pi^{0}$ ratio as well as the nuclear modification factor, making it a dominant source of uncertainty for the respective PCM measurements.

Trigger normalization: The uncertainty associated with the trigger rejection factor has been determined by varying the lower bound of the plateau fitting range. As seen in Figure 4.4 the plateau region is subject to small fluctuations which result in the values listed in Table 2 for the trigger rejection factors of the EMCal triggers in pp and $\mathrm{p}-\mathrm{Pb}$. Uncertainties of $4-5 \%$ depending on the trigger are found from the fitting range variations. This uncertainty also cancels fully in the $\eta / \pi^{0}$ ratio as the ratio itself is calculated individually for each trigger.

Efficiency: For the pp analysis, two different Monte Carlo generators are used in order to determine the correction factors. The difference between the generators is considered as systematic uncertainty which is on the order of $1-2 \%$ depending on $p_{\mathrm{T}}$ and similar for both meson spec- 
tra. Furthermore, the trigger emulation using the trigger maker depends on optimized starting values for the trigger turn-on as well as the width of the turn-on. As the respective parameters need to be optimized by hand, a systematic uncertainty is assigned which only affects the $p_{\mathrm{T}}$ bins of the spectra that are affected by clusters close to the trigger turn on. This uncertainty is up to $4 \%$ for the lowest $p_{\mathrm{T}}$ bins of the triggered spectra due to the strong change in shape of the reconstruction efficiency around the trigger turn-on. As $\pi^{0}$ and $\eta$ mesons rely on different cluster energies at the same meson $p_{\mathrm{T}}$, this uncertainty does not cancel fully in the $\eta / \pi^{0}$ ratio.

Resolution: For the merged cluster analysis a crucial component is the energy resolution of the clusters which is strongly affected by particle overlaps especially at high $p_{\mathrm{T}}$. As the simulation does not fully reproduce particle multiplicities as well as the parton shower inside the jet as present in data, we have to assume an energy resolution bias of the EMCal clusters. A toy particle decay simulation is used in order to assign an uncertainty for this resolution effect. This simulation employs a parametrization of the measured $\pi^{0}$ spectrum as well as resolution matrices for three different event classes; events with no particle overlaps within $R<0.05$ around generated neutral pions in the EMCal and DCal acceptance, events with 12 allowed overlaps, and events with $>2$ overlaps in the same radial cone. Each of these classes presents a different $p_{\mathrm{T}}$-dependent energy resolution of the merged pion clusters as seen in Figure 6.24 (top) with a visible reconstructed energy loss in the zero overlap class and an up to $20 \%$ higher reconstructed energy in the $>2$ overlap class. Depending on $p_{\mathrm{T}}$ the composition of these event classes changes with the zero overlap class being dominant at low momentum with about $70 \%$ and the high overlap class being dominant at high $p_{\mathrm{T}}$ with $70 \%$ where large amounts of injet particles can overlap with the $\pi^{0}$ cluster. The systematic uncertainty to account for the energy resolution is determined by varying the composition of the three overlap classes which effectively modifies the in-jet particle production within the $R<0.05$ cone. Each class is modified by up to absolute $\pm 10 \%$ as indicated by the bands in the lower panel of Figure 6.24 and the generated $\pi^{0}$ spectrum based on the input parametrization is smeared according to the resolution matrices of with the randomly assigned over-

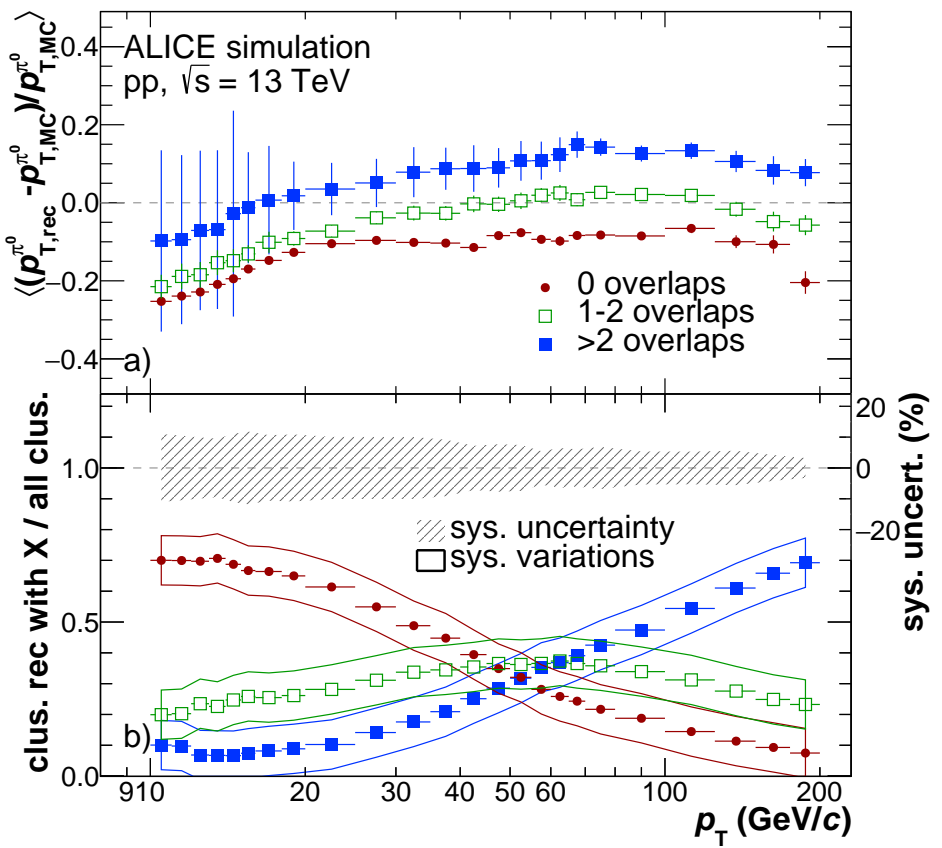

Figure 6.24: a) Mean cluster energy shift versus transverse momentum for three different neutral pion merged cluster types from Pyтнia8 Jet-Jet simulations at $\sqrt{s}=$ $13 \mathrm{TeV}$. Clusters with no overlapping particles within $R<0.05$ around the $\pi^{0}$ on Monte Carlo generator level are shown in red, while clusters with 1-2 overlapping particles are shown in green and clusters with more than two overlapping particles in blue. An increasing overlap of particles shifts the cluster energies to larger values. b) Fractions of clusters from the three overlap types in the total cluster sample are shown in the same colors. The bands indicate the systematic variations on the fractions which are applied in the Toy simulation in order to obtain the final systematic uncertainty shown in the shaded gray band versus transverse momentum. 
lap class based on their fractional probability distribution. This amounts to an uncertainty on the $\mathrm{mEMC} \pi^{0}$ spectrum of up to $10 \%$ at low $p_{\mathrm{T}}$ and about $5 \%$ at $p_{\mathrm{T}}=200 \mathrm{GeV} / c$.

For the final systematic uncertainty on the spectra, the uncertainties from the individual sources are added in quadrature and the resulting relative systematic uncertainties are shown in Figure 6.23 in the right panel for the different reconstruction techniques. A $p_{\mathrm{T}}$ dependent overview of the individual sources is shown in the appendix in Figure A.7 and Figure A.8 for the $\pi^{0}$ and $\eta$ spectra, the $\eta / \pi^{0}$ ratio and the nuclear modification factors. While statistical uncertainties, as shown in Figure 6.23 (left), in the intermediate $p_{\mathrm{T}}$ region $(1-5 \mathrm{GeV} / c)$ are of the order of $1-2 \%$, systematic uncertainties for PCM are about 10\%, for PCM-EMC about $7 \%$ and for EMC about $8 \%$ dominated by the material budget and signal extraction uncertainties. For high $p_{\mathrm{T}}$ where the EMCal triggered data enters, the uncertainties generally increase due to the substantial additional uncertainty coming from the trigger rejection factor.

\subsection{Correction for finite bin width}

The measurements of the neutral meson spectra as well as the $\eta / \pi^{0}$ ratio are performed with a finite width in the $p_{\mathrm{T}}$ slicing, which changes as a function of momentum. Binning a steeply falling spectrum like those of the neutral mesons requires an additional correction to account for the incorrect association of the $p_{\mathrm{T}}$ value of the bin center with the actual yield at the given $p_{\mathrm{T}}[214]$. This correction can be applied in one of two ways; the data can either be shifted horizontally in $p_{\mathrm{T}}$ in order to shift the value from the bin center to the correct transverse momentum or the data can be shifted vertically (in $y$-direction) in order to give the correct measurement value at the $p_{\mathrm{T}}$ of the bin center.

The shifting is based on the assumption that a Levy-Tsallis [215] function is able to describe the shape of the underlying spectra and the data points can be shifted horizontally or vertically to coincide with the values of the underlying Tsallis distribution. The combined neutral meson spectra are shifted horizontally in $p_{\mathrm{T}}$-direction for the final results which are presented in the next section. The corresponding bin shift correction factors are shown in Figure $6.25(t o p)$ for the combined $\pi^{0}$ and $\eta$ meson spectra. For both mesons the correction factors are less than $2 \%$ depending on the bin width.

The $y$-direction shifting approach is chosen for the calculation of the $\eta / \pi^{0}$ ratio in order to preserve the bin centers for a straightforward calculation of the ratio. A shift in $p_{\mathrm{T}}$ direction would result in different $p_{\mathrm{T}}$ values for a given bin for the $\pi^{0}$ and $\eta$ meson as their spectral shapes differ, especially in the low momentum region. The bin-shifting cor-

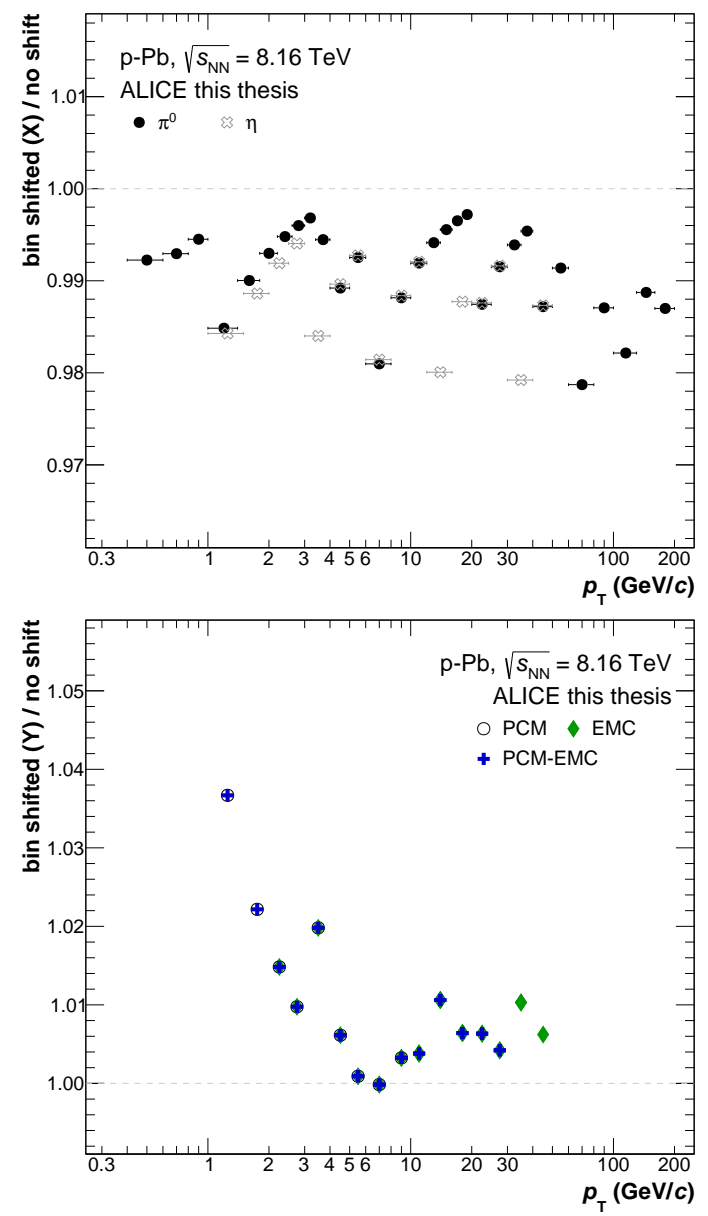

Figure 6.25: Top: Correction factor on the bin-center $p_{\mathrm{T}}$ for the combined $\pi^{0}$ (black) and $\eta$ (gray) meson spectra. Bottom: Bin shift correction in $y$-direction for the calculation of the $\eta / \pi^{0}$ ratio with PCM, PCMEMC and EMC. 
rection factors for the $\eta / \pi^{0}$ ratio reconstructed with PCM and PCM-EMC are plotted in Figure 6.25 (bottom), reaching values up to $4 \%$ at low $p_{\mathrm{T}}$ while at high $p_{\mathrm{T}}$ they are less than $2 \%$.

\subsection{Combination of spectra}

Similar to the combination of the different triggered spectra within one method, the last step is the combination of all different reconstruction techniques which are statistically uncorrelated but exhibit partial systematic uncertainty correlations. The resulting combined spectra profit from smaller total uncertainties in the overlapping regions as the central values are constrained by multiple reconstruction techniques. The combination is performed using the BLUE method taking into account the correlations between the methods which are mainly between the EMCal-based methods (PCM-EMC, EMC and mEMC). The standalone methods PCM, EMC and PHOS were found to be fully uncorrelated with each other. The weights for the different methods, which already include the estimated uncertainty correlations, are shown in Figure 6.26 for the $\pi^{0}$ and $\eta$ meson while the same figure for the $\eta / \pi^{0}$ ratio can be found in the appendix in Figure A.11. For the combination of the spectra a common $p_{\mathrm{T}}$ binning between all methods was determined in order to maximize the overlap of the different reconstruction techniques. This is necessary as the combination procedure can only be performed if a data point is measured with at least two reconstruction techniques in the same $p_{\mathrm{T}}$ interval. The weights show that the PHOS method is the dominant $\pi^{0}$ measurement up to $p_{\mathrm{T}} \approx 30 \mathrm{GeV} / c$ due to the small associated uncertainties. In the intermediate $p_{\mathrm{T}}$ region from 2-6 GeV/c both PCM-EMC and EMC contribute about equally well with PCM-EMC gaining a comparably larger weight towards higher $p_{\mathrm{T}}$, which is attributed to the additional statistics from the EMCal triggers and the low impact of cluster merging on the method. At very high $p_{\mathrm{T}}, \mathrm{mEMC}$ is the only method able to measure the $\pi^{0}$ spectrum and therefore it carries the full weight. The appearance of negative weights in Figure 6.26 points to highly correlated data points [209] where the measurement would not affect the central value due to the high correlation with another measurement. For the $\eta$ meson, the PCM and PCM-EMC reconstruction techniques are dominant at low $p_{\mathrm{T}}$, while the PHOS and EMC spectra determine the high $p_{\mathrm{T}}$ part of the combination.

The combined neutral meson spectra are fitted with a two-component model (TCM) function, which was proposed in Ref. [216,216-218] by Bylinkin and Rostovtsev, by using the total uncertainties for each $p_{\mathrm{T}}$ bin. The fit function is described as:

$$
E \frac{\mathrm{d}^{3} \sigma}{\mathrm{d} p^{3}}=A_{\mathrm{e}} \exp \left(-E_{\mathrm{T}, \operatorname{kin}} / T_{\mathrm{e}}\right)+A\left(1+\frac{p_{\mathrm{T}}^{2}}{T^{2} n}\right)^{-n},
$$
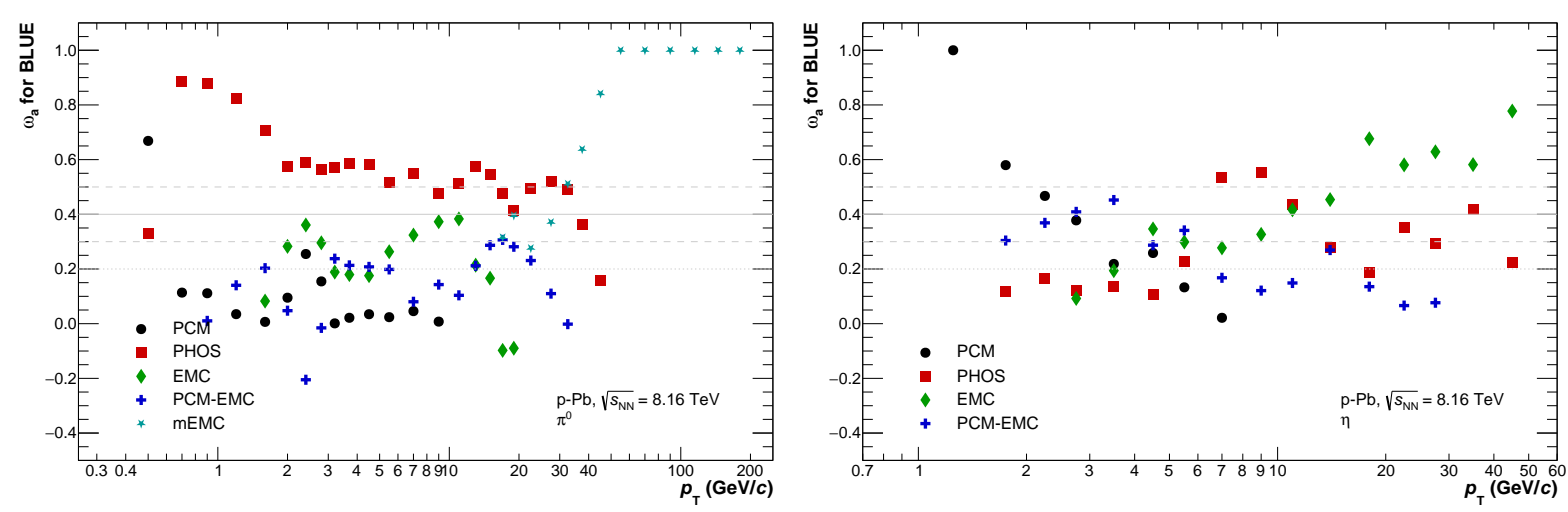

Figure 6.26: Weights used in the BLUE method for the combination of the different $\pi^{0}$ (left) and $\eta$ meson (right) spectra versus transverse momentum. 

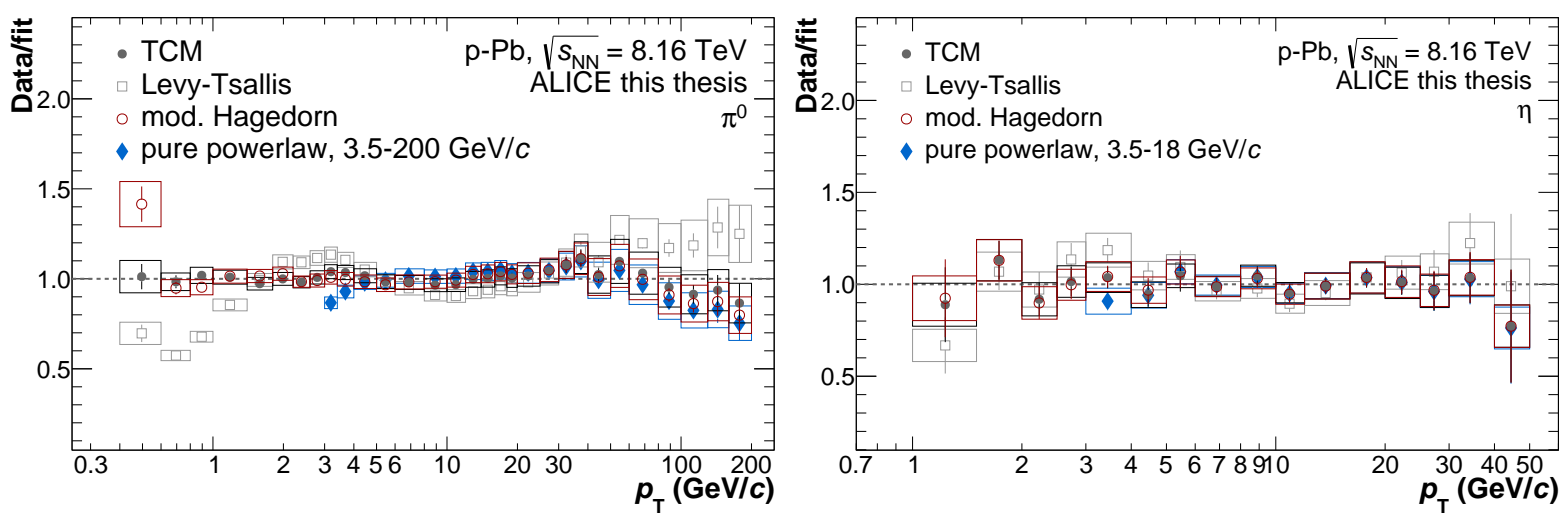

Figure 6.27: Ratio of the combined $\pi^{0}$ (left) and $\eta$ meson (right) cross section spectra in $\mathrm{p}-\mathrm{Pb}$ to four different fit functions. The comparison to a two-component model fit [217] is shown in gray circles while a Levy-Tsallis fit [215] comparison is shown in open gray squares. In addition, comparisons to a modified Hagedorn function [219] as well as a power-law for the high momentum region of $p_{\mathrm{T}}>3.5 \mathrm{GeV} / c$ is given. Similar plots for pp are given in the appendix in Figure A.12

where $E_{\mathrm{T}, \text { kin }}=\sqrt{p_{\mathrm{T}}^{2}+m^{2}}-m$ is the transverse kinematic energy with the $\pi^{0}$ or $\eta$ meson rest mass $m$ and $A_{\mathrm{e}}, A, T_{e}, T$ as well as $n$ are free parameters. The fit is able to reproduce the spectra over the full $p_{\mathrm{T}}$ range and its parameters are summarized in Tab. 9 for $\mathrm{p}-\mathrm{Pb}$ and $\mathrm{pp}$.

The comparison of the combined spectra to the TCM fits are shown in Figure 6.27 and Figure A.12 for $\mathrm{p}-\mathrm{Pb}$ and $\mathrm{pp}$, respectively. For both mesons, the spectrum can be described by this fit function within the total uncertainties over the full $p_{\mathrm{T}}$ range. In addition, comparisons to a Levy-Tsallis [215] distribution are shown which fails to describe the high $p_{\mathrm{T}}$ points but has proven to fairly well describe previously measured spectra at lower transverse momentum and collision energies $[195,206]$. In addition, a modified hagedorn function [219] according to

$$
E \frac{\mathrm{d}^{3} N}{\mathrm{~d} p^{3}}=A \cdot\left[\exp \left(a p_{\mathrm{T}}+b p_{\mathrm{T}}^{2}\right)+\frac{p_{\mathrm{T}}}{p_{0}}\right]^{-n}
$$

which was proposed by the PHENIX collaboration is used to describe the data and shows a similarly well description power as the TCM fit with an about $5-10 \%$ larger deviation from the data at high $p_{\mathrm{T}}$. Lastly, the data was fitted in the high momentum region of $p_{\mathrm{T}}>3.5 \mathrm{GeV} / c$ with a pure power-law function which compares fairly well to the data and allows the extraction of the spectral slope in the various systems as described in Section 6.6. As the TCM fit shows the best descriptive power for both the $\pi^{0}$ and $\eta$ meson it is used in all following comparisons to data or theory calculations.

Figure 6.28 shows the ratio of the different $\pi^{0}$ (left) and $\eta$ meson (right) spectra from the various reconstruction techniques with respect to the TCM fit, which allows for a comparison

\begin{tabular}{cc|cccccc}
\hline & TCM & $A_{e}\left(\mathrm{GeV}^{-2} c^{3}\right)$ & $T_{e}(\mathrm{GeV})$ & $A\left(\mathrm{GeV}^{-2} c^{3}\right)$ & $T(\mathrm{GeV})$ & $n$ & $\chi_{\text {red }}^{2}$ \\
\hline \hline $\mathrm{p}-\mathrm{Pb}$ & $\pi^{0}$ & $122.3 \pm 95.3$ & $0.093 \pm 0.015$ & $2.11 \pm 0.17$ & $0.649 \pm 0.011$ & $3.039 \pm 0.08$ & 0.45 \\
& $\eta$ & $(8.85 \pm 7.85) \times 10^{-3}$ & $0.722 \pm 0.117$ & $0.212 \pm 0.103$ & $0.784 \pm 0.083$ & $2.902 \pm 0.048$ & 0.35 \\
\hline & & $A_{e}\left(\mathrm{pb} \mathrm{GeV}^{-2} c^{3}\right)$ & $T_{e}(\mathrm{GeV})$ & $A\left(\mathrm{pb} \mathrm{GeV}^{-2} c^{3}\right)$ & $T(\mathrm{GeV})$ & $n$ & $\chi_{\text {red }}^{2}$ \\
\hline $\mathrm{pp}$ & $\pi^{0}$ & $(4.98 \pm 0.03) \times 10^{11}$ & $0.146 \pm 0.020$ & $(3.72 \pm 0.67) \times 10^{10}$ & $0.598 \pm 0.021$ & $3.042 \pm 0.010$ & 0.25 \\
& $\eta$ & $(4.52 \pm 32.40) \times 10^{8}$ & $0.217 \pm 0.211$ & $(2.95 \pm 1.20) \times 10^{9}$ & $0.801 \pm 0.069$ & $3.012 \pm 0.036$ & 0.38 \\
\hline
\end{tabular}

Table 9: Parameters of the fits to the $\pi^{0}$ and $\eta$ invariant differential yield and cross sections using the TCM fit $[217,218]$ from Eq. 34 for pp, $\sqrt{s}=8 \mathrm{TeV}$ and p-Pb, $\sqrt{s_{\mathrm{NN}}}=8.16 \mathrm{TeV}$. 

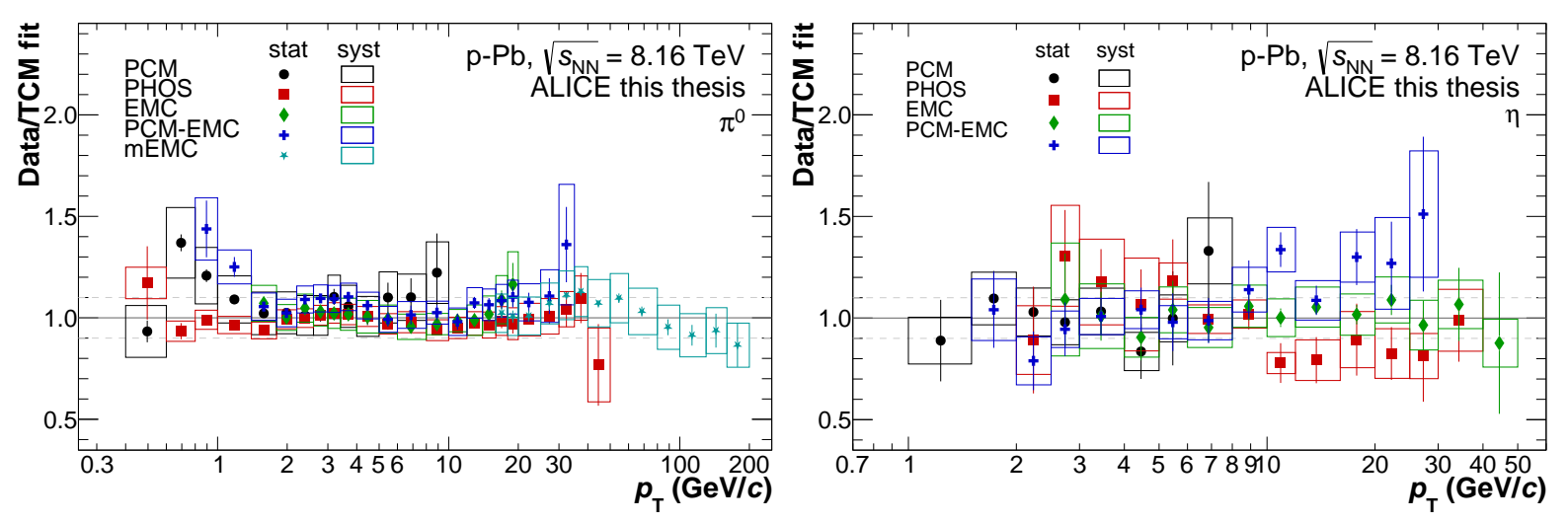

Figure 6.28: Ratio of the individual $\pi^{0}$ (left) and $\eta$ meson (right) cross section spectra in $\mathrm{p}-\mathrm{Pb}$ to the two-component model fit of the respective combined spectra.

between the individual results from each technique. Similar comparisons for pp are shown in the appendix in Figure A.13. For both mesons, the individual spectra are shown in the full range in which they were measured. This can exceed the range in which they were considered for the combination due to changes in the $p_{\mathrm{T}}$ binning to account for method-dependent changes in available statistics and background. All methods agree within their total uncertainties in the overlapping regions, however possible tensions at low momentum between PCM, PCM-EMC and PHOS are visible, which are however still within $2 \sigma$ of the total uncertainties. Furthermore, this showcases the excellent performance of the mEMC method, which extends the $p_{\mathrm{T}}$ reach of the $\pi^{0}$ invariant mass based measurements by an order of magnitude up to $200 \mathrm{GeV} / c$. For the $\eta$ meson, uncertainties are generally large but a similar agreement between all methods is found over the full signal extraction region which covers a $p_{\mathrm{T}}$ range from $1-50 \mathrm{GeV} / c$.

The individual $\eta / \pi^{0}$ ratio measurements are also compatible with each other as shown in the appendix in Figure A.14. However, due to the already significant uncertainties on the $\eta$ meson measurements, the $\eta / \pi^{0}$ ratios show large uncertainties and fluctuations over the full $p_{\mathrm{T}}$ range which are mostly removed in the combined spectrum as shown later in Section 6.6.2. 


\subsection{Results}

This section presents the final results of the neutral meson analyses. The combined $\pi^{0}$ and $\eta$ meson spectra as well as the $\eta / \pi^{0}$ ratio are presented together with comparisons to theory calculations and spectra from comparable particle species. This is followed by the calculation of the nuclear modification factor $R_{\mathrm{pA}}$ and its comparison to theory calculations as well as a previous measurement at $\sqrt{s_{\mathrm{NN}}}=5.02 \mathrm{TeV}$ and charged hadron measurements from ATLAS and CMS. The results cover the largest transverse momentum range of any identified particle spectrum to date reaching up to $p_{\mathrm{T}}=200 \mathrm{GeV} / c$ for the neutral pion $p_{\mathrm{T}}$-differential invariant cross sections in $\mathrm{pp}$ and $\mathrm{p}-\mathrm{Pb}$ collisions as well as the respective nuclear modification factor measured at $\sqrt{s_{\mathrm{NN}}}=8.16 \mathrm{TeV}$.

\subsubsection{Spectra}

The $p_{\mathrm{T}}$-differential invariant cross sections are calculated as

$$
E \frac{\mathrm{d}^{3} \sigma}{\mathrm{d} p^{3}}=\frac{1}{p_{\mathrm{T}}} \frac{1}{R F \cdot \mathscr{L}_{\mathrm{int}}} \frac{1}{\epsilon} \frac{1}{\Gamma_{\mathrm{BR}}} \frac{N^{\pi^{0}(\eta)}}{\Delta p_{\mathrm{T}}}
$$

where $R F \cdot \mathscr{L}_{\text {int }}$ is the integrated luminosity scaled by the trigger rejection factor $(R F)$ based on the minimum bias trigger cross sections of $\sigma_{\mathrm{MB}}^{\mathrm{pPb}}=2.09 \pm 0.04 \mathrm{~b}$ for $\mathrm{p}-\mathrm{Pb}, \sigma_{\mathrm{MB}}^{\mathrm{Pbp}}=2.10 \pm 0.04 \mathrm{~b}$ for $\mathrm{Pb}-\mathrm{p}$ [179] and $\sigma_{\mathrm{MB}}^{\mathrm{pp}}=55.8 \pm 0.04 \mathrm{mb}$ for $\mathrm{pp}$ [178]. The pileup and secondary contamination corrected raw yield in the rapidity range $[-0.8,0.8]$ is given by $N^{\pi^{0}(\eta)}$ and the total correction factor by $\epsilon$ according to Figure 6.11. The branching ratios $\Gamma$ of the two-photon decay channels of the $\pi^{0}\left(\Gamma\left(\pi^{0} \rightarrow \gamma \gamma\right)=98.8 \%\right)$ and the $\eta$ meson $(\Gamma(\eta \rightarrow \gamma \gamma)=39.4 \%)$ enter the calculation as well as normalization factors [184]. The $p_{\mathrm{T}}$ in Equation 36 is the corrected $p_{\mathrm{T}}$ obtained from the bin shift in $x$ direction.

The differential invariant cross sections for the $\pi^{0}$ and $\eta$ meson in pp collisions at $\sqrt{s}=8 \mathrm{TeV}$ and $\mathrm{p}-\mathrm{Pb}$ collisions at $\sqrt{s_{\mathrm{NN}}}=8.16 \mathrm{TeV}$ are shown in Figure 6.29. The spectra are overlaid with their respective TCM fits indicated by the dotted gray lines according to Equation 34 with the parameters from Table 9. The data is compared to PyThIA8.2 predictions [220] using the well established MONASH 2013 tune [167] and next-to-leading order pQCD calculations [221-224] with different nuclear parton distribution functions (nPDF) [77, 225]. For the NLO calculations, the factorization, fragmentation and renormalization scales are given in a range of $0.5 p_{\mathrm{T}}<\mu<2 p_{\mathrm{T}}$ while variations of the $\mathrm{nPDF}$ replicas are not included.

As can be seen in Figure 6.29, the PythiA8.2 event generator provides predictions over the full $p_{\mathrm{T}}$ range as it includes soft and hard parton interactions including additional heavier particle decays that produce $\pi^{0}$ and $\eta$ mesons at low $p_{\mathrm{T}}$ which are also contained in the measured spectra. The Pythia8.2 predictions tend to overestimate the $\pi^{0}$ spectrum in the low momentum region by up to $60 \%$ while underestimating in the mid $p_{\mathrm{T}}$ range $(2-5 \mathrm{GeV} / c)$ by about $20 \%$. At very high transverse momentum, they are able to describe the spectrum and its slope within the uncertainties. This furthermore holds true for both, the EPPs16 [225] and the nCTEQ15 [77], nuclear PDFs that were used for the predictions and which show only a $5-10 \%$ difference from each other at very low $p_{\mathrm{T}}$. In the case of the $\eta$ meson predictions, a general underestimation of the data is observed between $5 \%$ and $30 \%$ depending on $p_{\mathrm{T}}$. This underestimation of strangeness in PythiAis also observed for other particles and was reduced by the Monash 2013 Tune, but still requires further constrains [167].

The NLO pQCD calculations fail to correctly describe soft parton interactions and thus the predictions can only be reliably calculated for $p_{\mathrm{T}}>2 \mathrm{GeV} / c$ for both mesons. A similar behavior of the NLO calculations is observed in pp and $\mathrm{p}-\mathrm{Pb}$ where the DSS14 fragmentation function [222] together with nCTEQ15 nPDF and CT10 PDF are used, respectively. In both systems, 


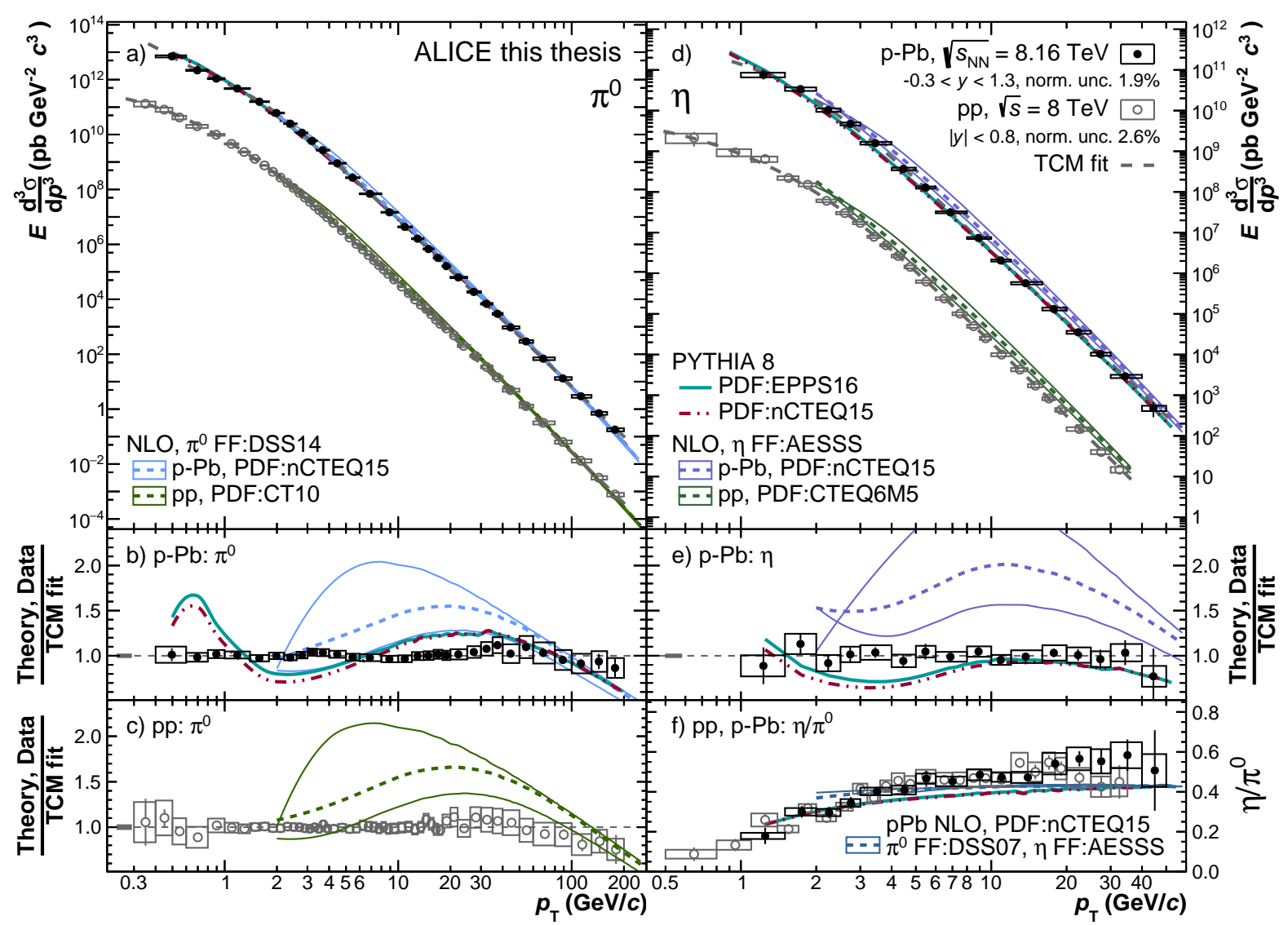

Figure 6.29: Neutral pion (a) and $\eta$ meson (d) differential invariant cross section for pp collisions at $\sqrt{s}=8 \mathrm{TeV}$ and $\mathrm{p}-\mathrm{Pb}$ collisions at $\sqrt{s_{\mathrm{NN}}}=8.16 \mathrm{TeV}$ overlaid with a TCM fit and NLO calculations [221-224] as well as PYTHIA8 [167,220] predictions employing different nPDFs [77, 225]. Statistical uncertainties are shown as vertical error bars and the systematic uncertainties are given as boxes. The ratios of the $\pi^{0}$ spectra in $\mathrm{p}-\mathrm{Pb}$ and $\mathrm{pp}$ collisions to the TCM fits are shown in panel (b) and (c), respectively, together with the ratios of the calculations to the fits. In panel (e) the ratio of the $\mathrm{p}-\mathrm{Pb} \eta$ spectrum to the TCM fit is shown together with the corresponding ratios of the calculations to the fit. In panel (f) the $\eta / \pi^{0}$ ratios in pp and p- $\mathrm{Pb}$ are compared to theory predictions. The overall normalization uncertainty is indicated as a solid gray box around unity.

the calculations generally overestimate the measured $\pi^{0}$ spectrum by $10 \%$ up to a factor of 2 depending on the factorization, fragmentation and renormalization scale.

For the $\eta$ meson, the nCTEQ15 nPDF for $\mathrm{p}-\mathrm{Pb}$ and the CTEQ6M5 [226] PDF for pp are used in conjunction with the AESSS [223] fragmentation function, which does not yet include constraints from LHC energy data. Due to the outdated fragmentation functions, the disagreement between data and NLO calculations is significantly larger than for the $\pi^{0}$ meson with an overestimation of $20 \%$ up to a factor of 3 depending on the scale. For both mesons, the $\mu=0.5 p_{\mathrm{T}}$ scale provides the best description of the data with the least deviations. 


\subsection{2 $\eta / \pi^{0}$ ratio}

An important measure for particle production is the relative abundance of one particle species compared to another. In the presented analysis, we can compare the spectrum of $\eta$ mesons to that of $\pi^{0}$ mesons via the $\eta / \pi^{0}$ ratio which has been measured across various collision systems and collision energies in the past $[196,206$, 227-232]. A universal behavior of this ratio for minimum bias collisions has been observed which approaches a constant value at high $p_{\mathrm{T}}$. The $\eta / \pi^{0}$ ratio in pp collisions at $\sqrt{s}=8$ $\mathrm{TeV}$ and $\mathrm{p}-\mathrm{Pb}$ collisions at $\sqrt{s_{\mathrm{NN}}}=$ $8.16 \mathrm{TeV}$ is shown in Figure 6.30 together with theory predictions from PYTHIA 8.2 as well as pQCD NLO

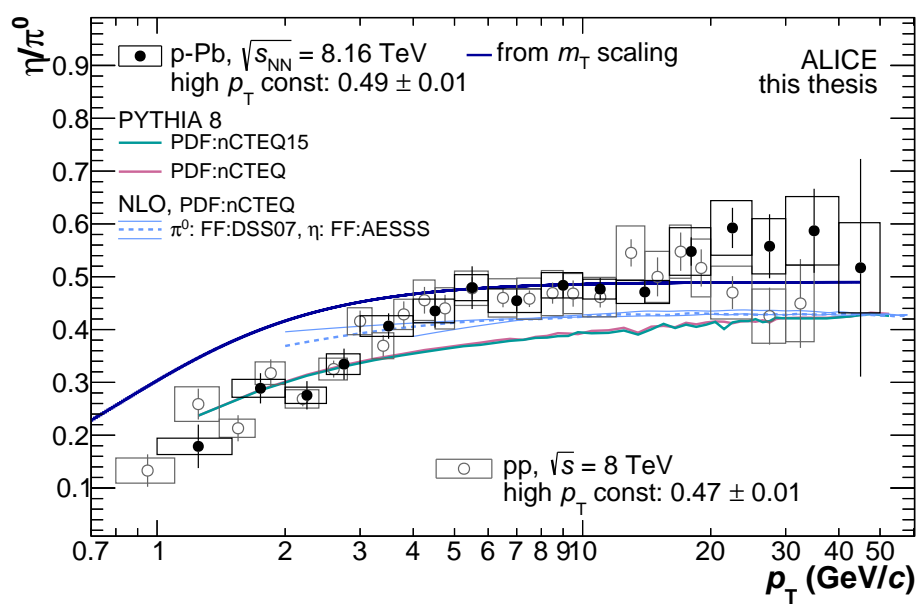

Figure 6.30: Comparison of the measured $\eta / \pi^{0}$ ratios in pp collisions at $\sqrt{s}=8 \mathrm{TeV}$ and $\mathrm{p}-\mathrm{Pb}$ collisions at $\sqrt{s_{\mathrm{NN}}}=8.16 \mathrm{TeV}$ to PYTHIA8.2 and pQCD NLO theory calculations. calculations using nCTEQ15 nPDFs together with consistent fragmentation functions of DSS07 [233] for the $\pi^{0}$ and AESSS for the $\eta$ meson. Similar to the data, also the theory predictions experience strong cancellations of uncertainties in this ratio where predictions of the same $\mu$ scale have been divided in the range of $0.5 p_{\mathrm{T}}<\mu<2 p_{\mathrm{T}}$. While these predictions, which are based on pre-LHC fragmentation functions for the $\eta$ meson, fail to describe the individual spectra, they agree within uncertainties with the measured particle ratio. In addition, the Pyтнia8.2 predictions tend to underpredict the ratio by about $25 \%$ for both $\mathrm{pp}$ and $\mathrm{p}-\mathrm{Pb}$ which has also been observed in measurements at lower center-of-mass energies [195] and stems from the overall underestimation of the strangeness production in Pythia [167]. The difference between data and the Pythia8.2 prediction gives rise to the correction of the $\eta$ meson contamination in the mEMC analysis as described in Section 6.2.1. In addition to theory comparisons, also the transverse mass $\left(m_{\mathrm{T}}\right)$ scaling prediction is provided in Figure 6.30 where the $\eta$ meson spectrum is calculated based on the TCM fit parametrization of the $\pi^{0}$ meson using $m_{\mathrm{T}}$-scaling [92,234]. This scaling relation, proposed by Hagedorn in 1965, assumes that hadron production can be described by a universal scaling law, characterized by a statistical-thermodynamical model [235]. It should therefore be possible to describe particle spectra by an exponential function, where the slope parameter is a universal value, if they are expressed as a function of $m_{\mathrm{T}}=\sqrt{p_{\mathrm{T}}^{2}+m_{0}^{2}}$ where $m_{0}$ is the particles rest mass. This relation holds true for measurements at low center-of-mass energies [227,236-239] where results from PHENIX additionally confirmed that meson and baryon spectra must be scaled separately. Measurements at LHC energies $[195,196,234]$ have shown this relation to be violated for light mesons. The respective calculated $\eta / \pi^{0}$ ratio is shown as a blue line which, by construction, describes the high $p_{\mathrm{T}}$ region where $m_{\mathrm{T}} \approx p_{\mathrm{T}}$ and where spectra are assumed to follow a power-law with similar slope. As has been shown in Ref. [234], this scaling relation overestimates the low momentum region by a large amount with more than $4 \sigma$. This is explained by the fact that the inclusive neutral pion measurement used for the scaling contains contributions from decays of heavy resonances or other strong decays which also includes the $\eta$ meson. As concluded from Ref. [234], the neutral pion should be avoided for $m_{\mathrm{T}}$ scaling of heavier particles where a reasonably well low $p_{\mathrm{T}}$ description is desired. Instead heavier particles like charged kaons should be used for the calculation as they 

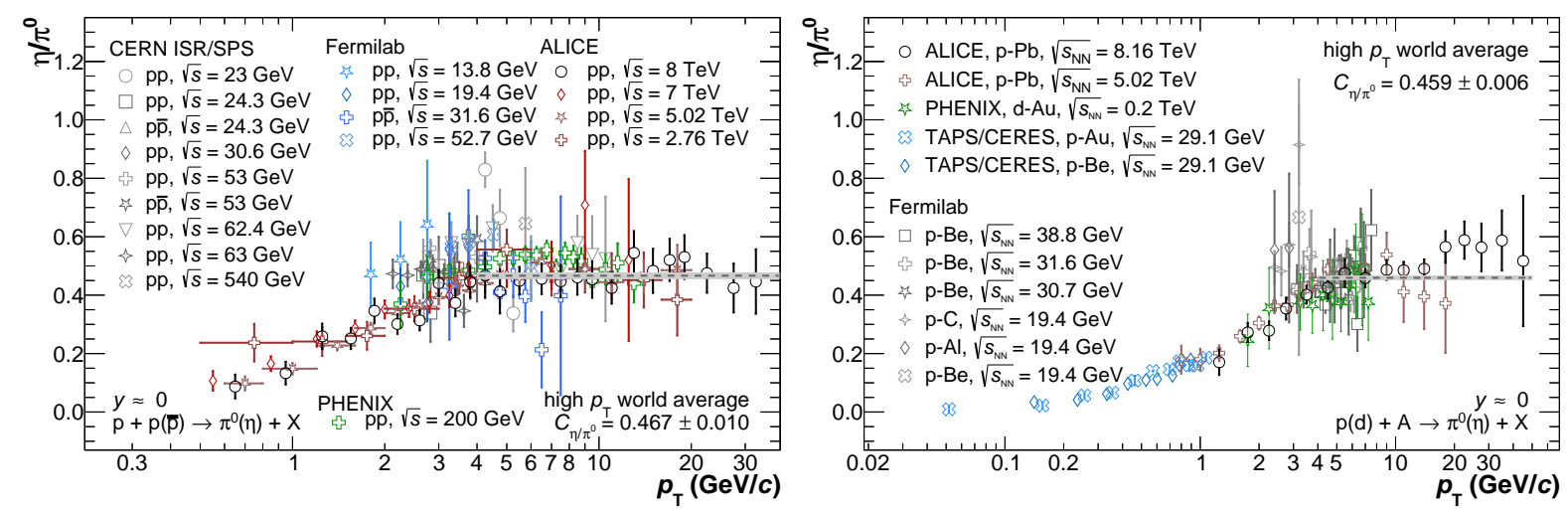

Figure 6.31: Left: Global fit of the $\eta / \pi^{0}$ ratio from various measurements performed at the LHC [195, 206], the ISR [240-245], the SPS [246-249], at the Fermilab Tevatron [250,251] and at RHIC [230] in pp and $\mathrm{p} \overline{\mathrm{p}}$ collisions. Right: Global fit of the $\eta / \pi^{0}$ ratio on measurements from TAPS/CERES [252], Fermilab [253-255], PHENIX [230] and ALICE [88, 196,256] in p(d)A collisions at various center-of-mass energies. Vertical lines present total uncertainties of the data.

are much less affected by feed-down. In order to compare the relative abundances between pp and $\mathrm{p}-\mathrm{Pb}$, the ratio is fitted with a constant for $p_{\mathrm{T}}>4 \mathrm{GeV} / c$ in the plateau region which results in values of $C_{\mathrm{pp}}^{\eta / \pi^{0}}=0.47 \pm 0.01$ and $C_{\mathrm{pPb}}^{\eta / \pi^{0}}=0.49 \pm 0.01$, respectively. The new measurements of the $\eta / \pi^{0}$ ratios are in addition used for an updated determination of the global fit value of the $\eta / \pi^{0}$ ratio. The global fit for $\mathrm{pp}$ and $\mathrm{p} \overline{\mathrm{p}}$ collisions is obtained via a constant fit above 4 $\mathrm{GeV} / c$ on data points at different collision energies from the LHC $[195,206]$, the ISR [240-245], the SPS [246-249], from the Fermilab Tevatron [250,251] and from RHIC [230] measurements as shown in Figure 6.31 (left). The resulting value of $C_{\text {global }}^{\eta / \pi^{0}}=0.467 \pm 0.010$ is consistent with each individual measurement within uncertainties. The ALICE measurements at $\sqrt{s}=2.76,5.02,7$ and $8 \mathrm{TeV}$ provide strong constraints in the high $p_{\mathrm{T}}$ plateau region as they are the only available measurements with small total uncertainties.

The global fit for $\mathrm{p}(\mathrm{d})-\mathrm{A}$ collision data is made on a set of data from combined efforts of TAPS and CERES [252] as well as data from Fermilab [253-255], PHENIX [230] and ALICE [88] including the new $\mathrm{p}-\mathrm{Pb}$ measurement at $\sqrt{s_{\mathrm{NN}}}=8.16 \mathrm{TeV}$ presented in this thesis. A constant fit for $p_{\mathrm{T}}>4 \mathrm{GeV} / c$ yields a value of $C_{\text {global }}^{\eta / \pi^{0}}=0.459 \pm 0.006$ with $\chi^{2} / \mathrm{NDF}=1.09$. The inclusion of the ALICE p-Pb measurements at $\sqrt{s_{\mathrm{NN}}}=5.02$ and $8.16 \mathrm{TeV}$ leads to a reduction of the relative uncertainty on the global fit value from $1.7 \%$ down to $1.3 \%$ while further constraining the central value. 


\subsubsection{Nuclear modification factor}

The main result of this thesis is the measurement of the nuclear modification of inclusive $\pi^{0}$ and $\eta$ meson production in $\mathrm{p}-\mathrm{Pb}$ compared to $\mathrm{pp}$ collisions. The difference in the particle production between both systems is quantified by measuring the nuclear modification factor according to

$$
R_{\mathrm{pA}}=\frac{\mathrm{d}^{2} \sigma_{\mathrm{pPb}}^{\pi^{0}, \eta} / \mathrm{d} p_{\mathrm{T}} \mathrm{d} y_{\mathrm{cms}}}{A_{\mathrm{Pb}} \times \mathrm{d}^{2} \sigma_{\mathrm{pp}}^{\pi^{0}, \eta} / \mathrm{d} p_{\mathrm{T}} \mathrm{d} y_{\mathrm{cms}}},
$$

where $\mathrm{d}^{2} \sigma / \mathrm{d} p_{\mathrm{T}} \mathrm{d} y_{\text {cms }}$ are the $\pi^{0}$ or $\eta$ meson cross sections in $\mathrm{p}-\mathrm{Pb}$ and pp collisions evaluated at the same center-of-mass energy and in the same kinematic range. The scaling of the pp reference measurement is done using $A_{\mathrm{Pb}}=208$ which is the nuclear mass number of lead. As explained in Section 2.3.3, the nuclear modification factor is expected to be unity at high $p_{\mathrm{T}}$ in minimum bias $\mathrm{p}-\mathrm{Pb}$ collisions due to the predicted absence of nuclear effects. A suppression at high $p_{\mathrm{T}}$ would require significant energy losses in a hadron gas or quark-gluon plasma which are currently only expected to be present in heavy-ion collisions or possibly in very high multiplicity $\mathrm{p}-\mathrm{Pb}$ collisions. In order to correctly calculate $R_{\mathrm{pA}}$, the pp $\sqrt{s}=8 \mathrm{TeV}$ reference is required to be scaled to the same center-of-mass energy and rapidity window as in the $\mathrm{p}-\mathrm{Pb} \sqrt{s_{\mathrm{NN}}}=8.16 \mathrm{TeV}$ measurement. The scaling relation is determined using PythiA8.2 with the Monash2013 tune. It is calculated via the ratio of $\pi^{0}$ meson spectra from both center-of-mass energies and rapidity window cases. Figure 6.32 shows these ratios accounting only for the difference in center-ofmass energy (gray points) and with the additional rapidity window difference (blue points). For comparison, a further ratio considering only the forward direction is shown (red points). In addition, NLO calculations using the CT14 PDF were used to check the behavior at high $p_{\mathrm{T}}$ and are also found to be consistent with the PYTHIA-based ratio. The scaling function is obtained from the ratio of TCM fits that were made on the individual spectra in order to achieve the best possible description of the ratio. The function is shown in Figure 6.32 as dotted blue line and presents a small correction with at most $3 \%$ for the highest $p_{\mathrm{T}}$ bin.

The nuclear modification factor in $\mathrm{p}-\mathrm{Pb}$ collisions at $\sqrt{s_{\mathrm{NN}}}=8.16 \mathrm{TeV}$ is shown in Figure 6.33 for the $\pi^{0}$ (black) and the $\eta$ meson (gray). The figure shows the fully combined $R_{\mathrm{pA}}$ for both mesons obtained from the combination of the individual measurements using PCM, PCM-EMC, EMC and mEMC and PHOS. A comparison of the individual measurements is provided in the appendix in Figure A.15 and Figure A.16 for the $\pi^{0}$ and $\eta$ meson, respectively. Furthermore, the weights used in the BLUE method combination procedure as described in Section 6.1.2 are given in the same figures which show the strength of each reconstruction technique depending on $p_{\mathrm{T}}$. The normalization uncertainty on the measurement of $3.4 \%$ is shown as a gray box in Figure 6.33 and subsequent figures. It stems from the uncertainty on the minimum bias trigger cross section determination in $\mathrm{pp}$ and $\mathrm{p}-\mathrm{Pb}$.

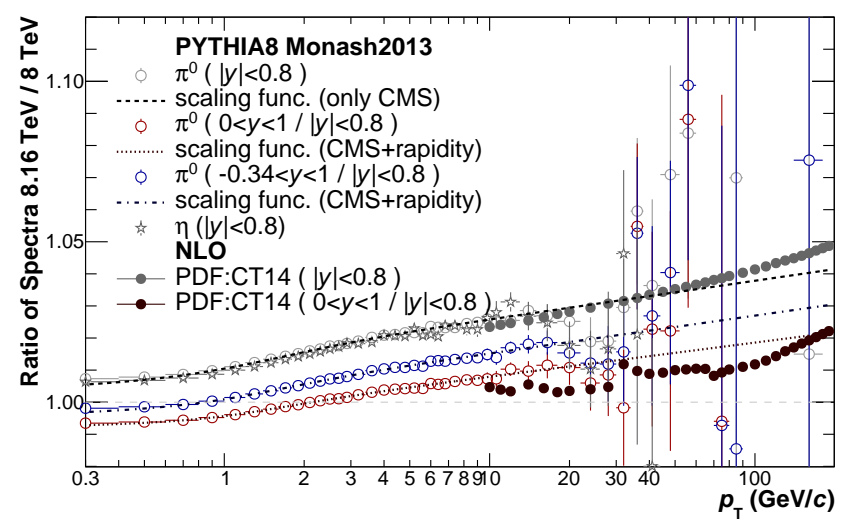

Figure 6.32: Ratio of $\pi^{0}$ meson spectra from Pythia8.2 with the Monash2013 tune as well as for NLO calculations using the CT14 PDF between the center-of-mass energies of $\sqrt{s_{\mathrm{NN}}}=8.16 \mathrm{TeV}$ and $\sqrt{s}=8 \mathrm{TeV}$ at mid rapidity $(|y|<0.8$ as well as boosted for the $\sqrt{s}=8.16 \mathrm{TeV}$ spectrum to $-0.3<y<1.3)$. The scaling function is the ratio of TCM fits on the individual spectra and shown as blue dotted line. 
As can be seen in Figure 6.33, the $R_{\mathrm{pA}}$ of both mesons shows a suppression for $p_{\mathrm{T}}<$ $10 \mathrm{GeV} / c$ which becomes much stronger for $p_{\mathrm{T}}<3 \mathrm{GeV} / c$. The modification of the low $p_{\mathrm{T}}$ region is expected as a result of multiple scattering effects in the nucleus as well as the effect of flow, as described in Section 2.3.3.1. This furthermore can cause an enhancement at intermediate $p_{\mathrm{T}}$ in the $R_{\mathrm{pA}}$ which is predominantly observed as a strong effect for heavy particles as well as baryons. In the presented measurement, a local maximum is visible around $p_{\mathrm{T}} \approx 3$ $\mathrm{GeV} / c$ where the $R_{\mathrm{pA}}$ approaches unity and subsequently drops to a value of approximately 0.9 afterwards. The deviation from unity in the intermediate $p_{\mathrm{T}}$ region presents itself significant considering the uncertainty on the measurement as well as the normalization uncertainty. A previously published measurement in $\mathrm{p}-\mathrm{Pb}$ collisions at

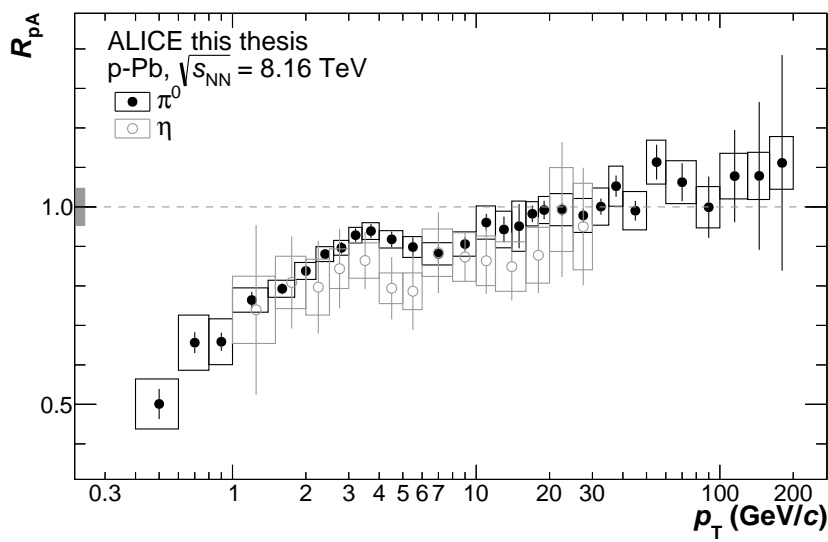

Figure 6.33: Nuclear modification factor of neutral pions (black) and $\eta$ mesons (gray) in $\mathrm{p}-\mathrm{Pb}$ collisions at $\sqrt{s_{\mathrm{NN}}}=8.16 \mathrm{TeV}$ combined from the individual measurements using the BLUE method. Statistical uncertainties are given as vertical bars while systematic uncertainties are shown as boxes. The global uncertainty due to the minimum bias trigger cross section uncertainty in $\mathrm{pp}$ and $\mathrm{p}-\mathrm{Pb}$ of $3.4 \%$ is indicated by the gray box.

large uncertainties in this region and thus could not observe a significant modification as seen in Figure 6.34 (middle). However, in this measurement an interpolated pp reference spectrum was employed as the measured $\pi^{0}$ and $\eta$ meson spectra in pp $\sqrt{s}=5.02 \mathrm{TeV}$ were not yet available. A re-analysis of the same data presented in Ref. [88] showed also for $\mathrm{p}-\mathrm{Pb} \sqrt{s_{\mathrm{NN}}}=5.02 \mathrm{TeV}$ a possible suppression at intermediate $p_{\mathrm{T}}$ due to reduced uncertainties from an improved interpolation reference.

\section{Comparison to other particle species}

The $R_{\mathrm{pA}}$ is in addition compared to measurements of charged hadrons in $\mathrm{p}-\mathrm{Pb} \sqrt{s_{\mathrm{NN}}}=5.02 \mathrm{TeV}$ by ALICE [257] and CMS [258] in Figure 6.34 (middle) where especially the latter measurement reaches up to $p_{\mathrm{T}}=125 \mathrm{GeV} / c$. It can be seen that the charged hadron $R_{\mathrm{pA}}$ is visibly larger compared to the neutral pions by about $10 \%$ for $p_{\mathrm{T}}<10 \mathrm{GeV} / c$ which can be explained by the much stronger Cronin effect causing an enhancement for the included heavy protons and kaons in the measurement. Furthermore, at very high $p_{\mathrm{T}}$ the CMS measurements shows a possible enhancement of about $10 \%$ which is not observed for the neutral pion measurement that is consistent with unity within uncertainties. The slight enhancement in the CMS measurement is considered to be an anti-shadowing effects, which leads to an overall harder spectrum [258]. Comparisons to measurements of other identified particles like charged pions or charged kaons were not possible due to the lack of reference measurements in pp $\sqrt{s}=8 \mathrm{TeV}$ while the individual particle spectra in $\mathrm{p}-\mathrm{Pb}$ collision data have currently been measured only in a narrow low $p_{\mathrm{T}}$ region [259].

\section{Comparison to theory predictions}

Figure 6.34 shows comparisons of the $\pi^{0}$ nuclear modification factor to NLO pQCD calculations, Color-Glass-Condensate (CGC) predictions and cold nuclear matter energy loss (FCEL) calculations. The comparison to NLO pQCD calculations with different nuclear PDFs is based on spectra provided by Werner Vogelsang [226] who provided calculations using DSS14 [233] fragmentation functions together with CT10 [260,261] parton distribution functions for pp as 
well as two sets of calculations for p-Pb with EPPS16 [225] or nCTEQ15 [77] nuclear PDFs. For the calculation of $R_{\mathrm{pA}}$, the pp reference is scaled by $N_{\text {coll }}$ and the spectra using the same fragmentation and factorization scale are divided. The uncertainty band shown in Figure 6.34 on the NLO calculations is obtained from separately dividing the $\mu=0.5 p_{\mathrm{T}}$ and $\mu=2 p_{\mathrm{T}}$ scale calculations assuming that the scale is similar in both collision systems.

The calculations using the EPPS16 nPDFs are able to describe the data better at very low $p_{\mathrm{T}}$ compared to the calculations using nCTEQ15 nPDFS which underestimate the data by up to $20 \%$. At high $p_{\mathrm{T}}$ both calculations predict an excess of approximately $5 \%$ which is consistent with the data within uncertainties. A very good description of the data is achieved with the CGC predictions in the region where the calculations are provided $\left(1<p_{\mathrm{T}}<6 \mathrm{GeV} / c\right)$ [262]. These predictions are based on small- $x$ running coupling Balitsky-Kovchegov (BK) evolution [263,264] of the dipole-nucleus scattering amplitude which is obtained via fitting of the non-perturbative initial condition to the HERA structure function data. In addition, a generalization from proton to nucleus is introduced using the optical Glauber model. As a result, these predictions don't have free parameters to describe the nuclear structure. Based on the approach for the predictions, the $R_{\mathrm{pA}}$ approaches unity at high $p_{\mathrm{T}}$. In order to obtain the final prediction, the so-called $k_{\mathrm{T}}$ factorization is used where the proton and nucleus are considered to be dense sheets of CGC. With this, the gluon production is calculated with subsequent usage of the DSS fragmentation function to describe the gluon to pion hadronisation.

The calculation that considers fully coherent energy loss (FCEL) effects on the light hadron production is shown in magenta in Figure 6.34 for the $\pi^{0}$ meson $R_{\mathrm{pA}}$ [265]. This calculation is an FCEL baseline prediction which only contains FCEL effects leaving out further modifications from nPDFs, saturation or the Cronin effect. Furthermore, the calculations show no difference between light hadron species like pions, $\eta$ mesons or protons. Any species dependence is covered by the variations on the mean fragmentation variable $z=0.7 \pm 0.2$. The calculation is only provided for the $g g \rightarrow g g$ channel which is dominant at midrapidity and nearly unaffected by a possible inclusion of the QCD Compton scattering process $(q g \rightarrow q g)$. The FCEL effect scales antiproportional to the hard scale or $p_{\mathrm{T}}$ leading to a gradual reduction of the effect for higher momenta where the hard scale becomes large. Therefore, the predictions become consistent with unity for $p_{\mathrm{T}}>30 \mathrm{GeV} / c$. The calculations show a good agreement with the data from low to high $p_{\mathrm{T}}$ covering the suppression at low $p_{\mathrm{T}}$ within uncertainties, but overestimating the intermediate momentum region where the data presents an $R_{\mathrm{pPb}} \approx 0.9$.

A comparison between the $R_{\mathrm{pA}}$ measured in $\mathrm{p}-\mathrm{Pb} \sqrt{s_{\mathrm{NN}}}=8.16 \mathrm{TeV}$ and $\mathrm{p}-\mathrm{Pb} \sqrt{s_{\mathrm{NN}}}=5.02 \mathrm{TeV}$ shown in the bottom panel of Figure 6.34 presents a possibly stronger suppression of particle production in the higher center-of-mass energy system. The ratio of the nuclear modification factors can be fitted with a linear function in the region $1<p_{\mathrm{T}}<20 \mathrm{GeV} / c$ where overlapping data is available resulting in a constant value of $0.93 \pm 0.01$. This possible $7 \%$ increased suppression at $\sqrt{s_{\mathrm{NN}}}=8.16 \mathrm{TeV}$ is partially supported by NLO pQCD calculations predicting a 1-5\% stronger suppression for $p_{\mathrm{T}}<5 \mathrm{GeV} / c$ as well as by CGC calculations which predict a $2-5 \%$ smaller $R_{\mathrm{pA}}$. The comparison is, however, not significant enough as the $7 \%$ increased suppression is to be seen relative to a $6.2 \%$ normalization uncertainty. This uncertainty comes from the two separate measurements and is strongly affected by the uncertainty on the interpolated pp spectrum, which is used as the $\sqrt{s_{\mathrm{NN}}}=5.02 \mathrm{TeV}$ reference. With the analysis of the $2016 \mathrm{p}-\mathrm{Pb} \sqrt{s_{\mathrm{NN}}}=5.02 \mathrm{TeV}$ data which provides an order of magnitude more minimum bias statistics as well as a measurement in pp $\sqrt{s}=5.02 \mathrm{TeV}$ this comparison can be vastly improved and uncertainties can be largely reduced. Unfortunately, these measurements were not available in time for further studies on this interesting topic. 


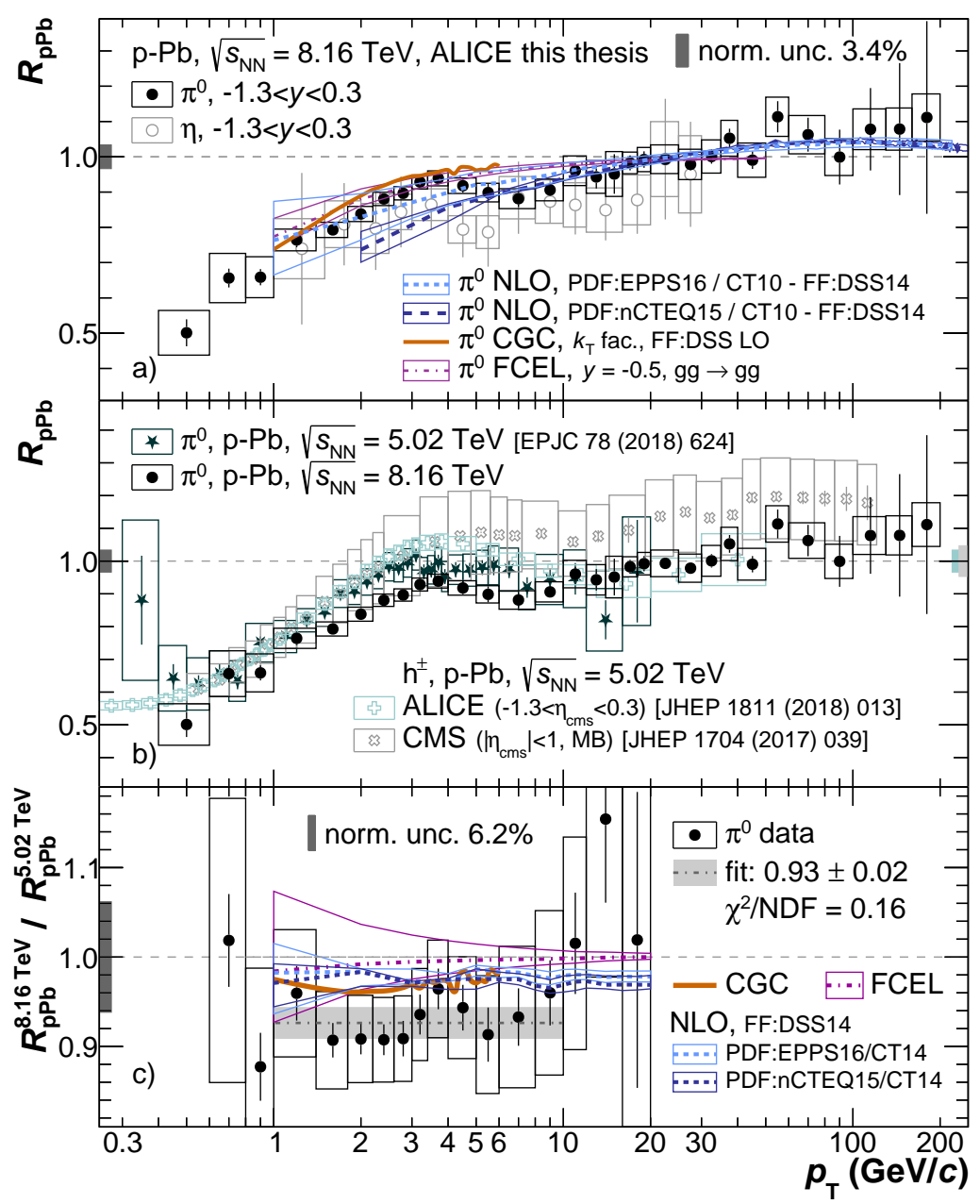

Figure 6.34: a) Combined nuclear modification factor of neutral pions (black) and $\eta$ mesons (gray) in $\mathrm{p}-\mathrm{Pb}$ collisions at $\sqrt{s_{\mathrm{NN}}}=8.16 \mathrm{TeV}$ from the individual measurements using the BLUE method. Statistical uncertainties are given as vertical bars while systematic uncertainties are shown as boxes. The global uncertainty due to the minimum bias trigger cross section uncertainty in pp and $\mathrm{p}-\mathrm{Pb}$ of $3.4 \%$ is indicated by the gray box. Data is compared to CGC [262], FCEL [265] and NLO pQCD calculations [226]. b) Comparison of the $\pi^{0}$ meson $R_{\mathrm{pA}}$ to various measurements at $\sqrt{s_{\mathrm{NN}}}=5.02 \mathrm{TeV}[232,257,258]$. c) The ratio of the $\pi^{0} R_{\mathrm{pA}}$ at $\sqrt{s_{\mathrm{NN}}}=8.16$ and $5.02 \mathrm{TeV}$ together with theory predictions $[262,265]$. 


\subsection{Meson summary and outlook}

First neutral pion and $\eta$ meson measurements in $\mathrm{p}-\mathrm{Pb}$ collisions at $\sqrt{s_{\mathrm{NN}}}=8.16 \mathrm{TeV}$ are made reaching the highest transverse momenta for identified particle spectra to date. An additional extension of a previously published spectrum in pp collisions at $\sqrt{s}=8 \mathrm{TeV}$ to the same high momentum is performed to supply a reference measurement for the calculation of the nuclear modification factor [196]. The large $p_{\mathrm{T}}$ reach is accomplished using multiple invariant mass-based reconstruction techniques and by exploiting shower overlaps in the EMCal together with a vastly improved understanding of the detector response. Both neutral meson spectra are overestimated by NLO pQCD calculations concluding that improved fragmentation functions, especially for the $\eta$ meson, are needed. The $\eta / \pi^{0}$ ratios in both collision systems show consistent values in the high $p_{\mathrm{T}}$ plateau region and are used in order to further constrain a global fit using inputs from various center-of-mass energies and collision systems.

The nuclear modification factors $R_{\mathrm{pA}}$ are determined from the $\mathrm{p}-\mathrm{Pb}$ spectrum and the scaled reference measurement providing data up to $p_{\mathrm{T}}=200 \mathrm{GeV} / c$. A suppression at low to intermediate momentum is determined consistent with predictions based on various models like gluon saturation or energy loss in cold nuclear matter. No modification of the yield is observed at high $p_{\mathrm{T}}$ contrary to a charged hadron measurement by CMS which resulted in a slight enhancement up to $p_{\mathrm{T}} \sim 120 \mathrm{GeV} / c$. A further comparison to a previous measurement by ALICE at $\sqrt{s_{\mathrm{NN}}}=5.02$ $\mathrm{TeV}$ shows a possibly stronger suppression in the higher center-of-mass energy system which is further supported by theory predictions. Further reductions in the statistical and systematic uncertainties of the measurements are necessary to draw definite conclusions from this comparison due to the currently limited precision.

In order to further study the nuclear modification of the particle production in $\mathrm{p}-\mathrm{Pb}$ collisions, the measurements can be repeated as a function of event multiplicity. Figure 6.35 presents the estimated lower bound for the statistical uncertainties of the mEMC reconstruction technique for $\pi^{0}$ mesons in in $\mathrm{p}-\mathrm{Pb}$ collisions at $\sqrt{s_{\mathrm{NN}}}=8.16 \mathrm{TeV}$ (left) and in $\mathrm{Pb}-\mathrm{Pb}$ collisions at $\sqrt{s_{\mathrm{NN}}}=5.02$ $\mathrm{TeV}$ (right) in various multiplicity percentile slices. It can be seen that such measurements in $\mathrm{p}-\mathrm{Pb}$ collisions could be achieved with statistical uncertainties of less than $5 \%$ up to $p_{\mathrm{T}} \approx 100$ $\mathrm{GeV} / c$. This level of precision could be reached in central and semi-central $\mathrm{Pb}-\mathrm{Pb}$ collisions only up to $p_{\mathrm{T}} \approx 60 \mathrm{GeV} / c$ which would still extend the currently available measurements by at least a factor of 2 and put stricter constraints on the observed high $p_{\mathrm{T}}$ suppression [256]. The main challenges for measurements in such high multiplicity environments are the correct treatment of background sources as well as the energy resolution of the reconstructed meson candidates. The precision of such differential measurements will therefore be limited by the systematic uncertainties associated with these effects.

Further studies can be performed by measuring neutral mesons in events that fulfill certain properties like a high or low sphericity [266] or jettiness [267]. First preliminary results by ALICE have been performed in pp collision data at $\sqrt{s}=5.02 \mathrm{TeV}$ and showed a hardening or softening of the $\pi^{0}$ and $\eta$ meson production spectra depending on the sphericity class as seen in Figure 6.36 (left). Moreover, measurements of the production of both neutral mesons inside of the cone of reconstructed jets showed a stronger suppression of the $\eta$ meson yield at low $p_{\mathrm{T}}$ compared to the neutral pion. This is visible in the $\eta / \pi^{0}$ ratio in Figure 6.36 (right) where the ratio is significantly lower in the in-jet case. Such measurements can provide insights into the dependence of particle production depending on the event shape and are first step towards highly differential analyses involving the light neutral mesons. 

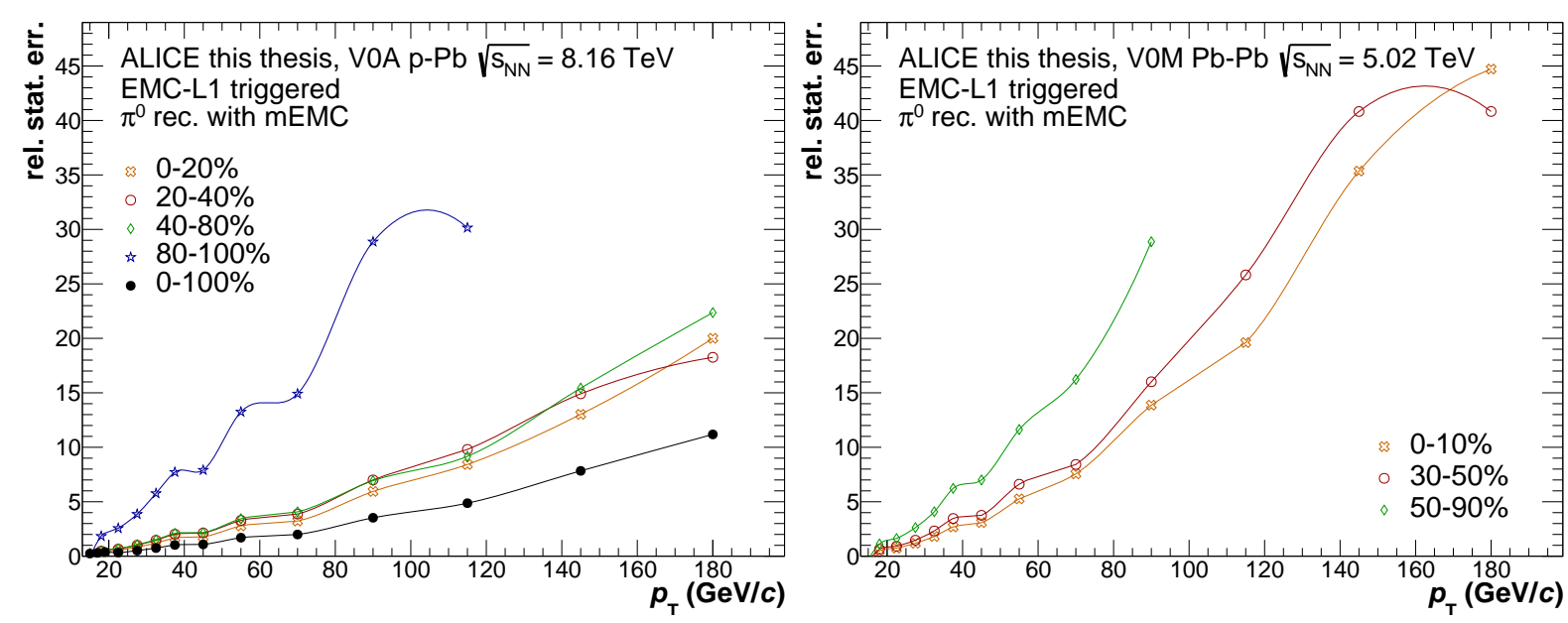

Figure 6.35: Relative statistical uncertainties on the raw $\pi^{0}$ meson yield reconstructed with the mEMC reconstruction technique in different V0A multiplicity and V0M centrality classes in $\mathrm{p}-\mathrm{Pb}$ collisions at $\sqrt{s_{\mathrm{NN}}}=8.16 \mathrm{TeV}$ (left) and in $\mathrm{Pb}-\mathrm{Pb}$ collisions at $\sqrt{s_{\mathrm{NN}}}=5.02 \mathrm{TeV}$ (right).
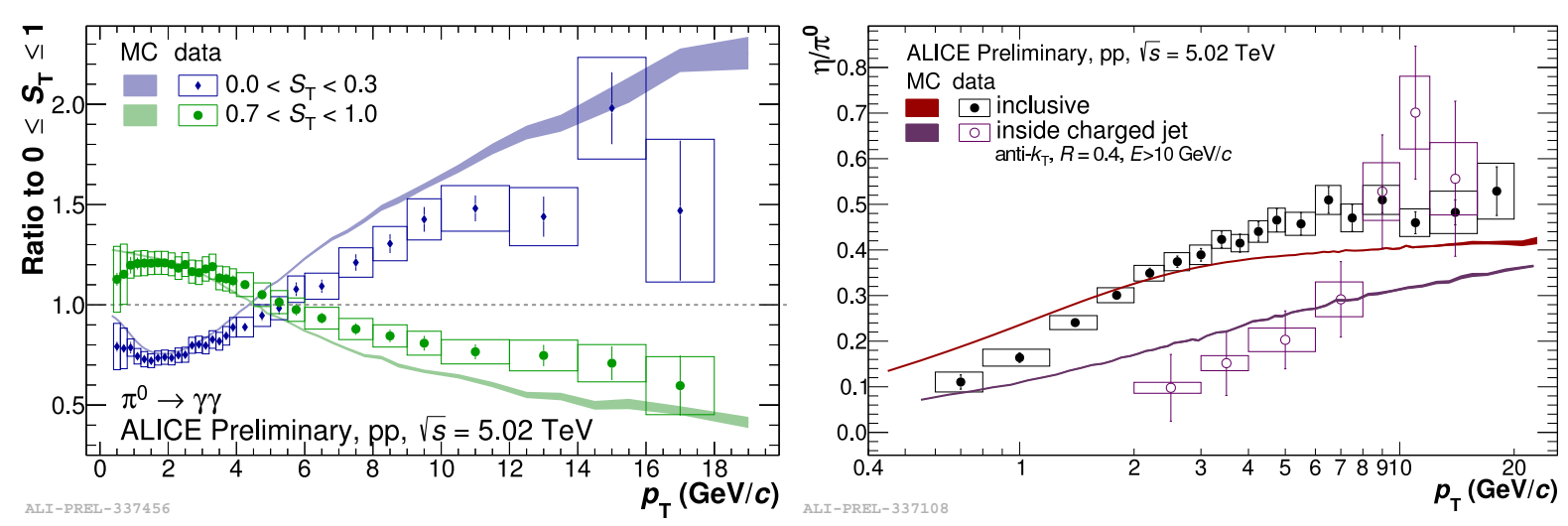

Figure 6.36: Left: Ratio of the neutral pion $p_{\mathrm{T}}$-differential invariant cross section measured in two sphericity event classes to the spectrum measured in events where sphericity can be determined. The data is compared to PyTHIA8.2 calculations, shown as bands. Right: Comparison of the inclusive $\eta / \pi^{0}$ ratio in pp collisions at $\sqrt{s}=5.02 \mathrm{TeV}$ to the same ratio where both mesons are required to be reconstructed within a charged jet of radius $R=0.4$ and an energy greater than $10 \mathrm{GeV} / c$. 


\section{Direct Photon Measurements}

In this chapter the multiplicity dependent inclusive and direct photon measurements in $\mathrm{p}-\mathrm{Pb}$ collisions at $\sqrt{s_{\mathrm{NN}}}=5.02 \mathrm{TeV}$ are presented. This analysis marks the first time that the multiplicity dependence of direct photon production is explored in proton-nucleus collisions with ALICE. The dominant photon reconstruction technique at low $p_{\mathrm{T}}$ is the photon conversion method, which can be used to search for a thermal photon signal in the soft photon region. Direct photons, as explained in the theory overview in Section 2.3.4, are defined as all photons that do not originate from particle decays. In this analysis, the direct photon production is extracted using the subtraction method according to Equation 9 which employs the measurement of the direct photon excess ratio $R_{\gamma}$ (Equation 10) as an intermediate step. This in turn requires an excellent knowledge of the decay photon background for which in a first step in Section 7.1 a particle decay simulation (cocktail simulation) is established. This simulation is based on parametrizations of measured spectra of particles that are responsible for the bulk of decay photons. The inclusive photon spectra require several corrections including the removal of out-of-bunch pileup, the subtraction of secondary photons, as well as acceptance, efficiency and resolution correction factors which are explained in Section 7.2. The systematic uncertainties of the inclusive and direct photon measurements are afterwards discussed in Section 7.3. Lastly the final results are combined in Section 7.4 and subsequently compared to theory predictions in Section 7.5. The results are in addition put into context with comparisons to pp and A-A collision measurements from various high energy experiments. The results of this analysis have been presented at Hard Probes 2018 and the corresponding proceedings of the conference can be found in Ref. [101]. The analysis therefore continues the efforts of the minimum bias [232] and multiplicity dependent [270] neutral meson measurements in $\mathrm{p}-\mathrm{Pb}$ collisions at $\sqrt{s_{\mathrm{NN}}}=5.02 \mathrm{TeV}$. For the final results, inputs from external analyses $[268,269]$ listed in Table 10 are combined with the PCM measurement. They provide a cross-check in the overlapping $p_{\mathrm{T}}$ region and extend the measurement to higher momentum.

\begin{tabular}{lcc}
\hline $\begin{array}{l}\text { reconstruction } \\
\text { method }\end{array}$ & $\begin{array}{c}p_{\mathrm{T}} \text { reach }(\mathrm{GeV} / c) \\
\text { for } \gamma_{\text {inc }} \text { and } R_{\gamma}\end{array}$ & reference \\
\hline PCM & $0.4-10$ & this thesis \\
PCM-EMC & $0.8-14$ & Analysis Note [268] \\
EMC & $2.0-14$ & Analysis Note [269] \\
PHOS & $1.0-32$ & Hard Probes 2018 \\
\hline Combination & $0.4-32$ & proceedings [101] \\
\hline
\end{tabular}

Table 10: Overview of available $\gamma_{\text {inc }}$ invariant yield and $R_{\gamma}$ measurements including their $p_{\mathrm{T}}$ range in $\mathrm{p}-\mathrm{Pb}$ collisions at $\sqrt{s_{\mathrm{NN}}}=5.02 \mathrm{TeV}$ for the four V0A multiplicity bins $0-20 \%, 20-40 \%, 40-60 \%$ and $60-100 \%$.

\subsection{Cocktail simulation}

The calculation of $R_{\gamma}$ (Equation 10) and subsequently of the direct photon spectrum requires a high precision estimate of the decay photon background. As the decay and direct photons can not be measured independently, we have to rely on simulations to determine the decay photon contribution. The general purpose event generator-based MC simulations used in the neutral meson analyses, as described in Section 4.3, are not suited for this determination. In particular, as the absolute abundances of the various particle species do not describe those in data with good enough precision. Instead cocktail simulations with the complete decay chain of all involved particles are utilized, where parametrizations of measured particle spectra are used as inputs in order to correctly describe the decay photon contributions. These cocktail simulations, albeit focused on the decay photons in this section, also provide the necessary inputs for the secondary photon and neutral pion corrections in Section 6.1.1.2 due to the full access to all intermediate decay steps in the generated output. 

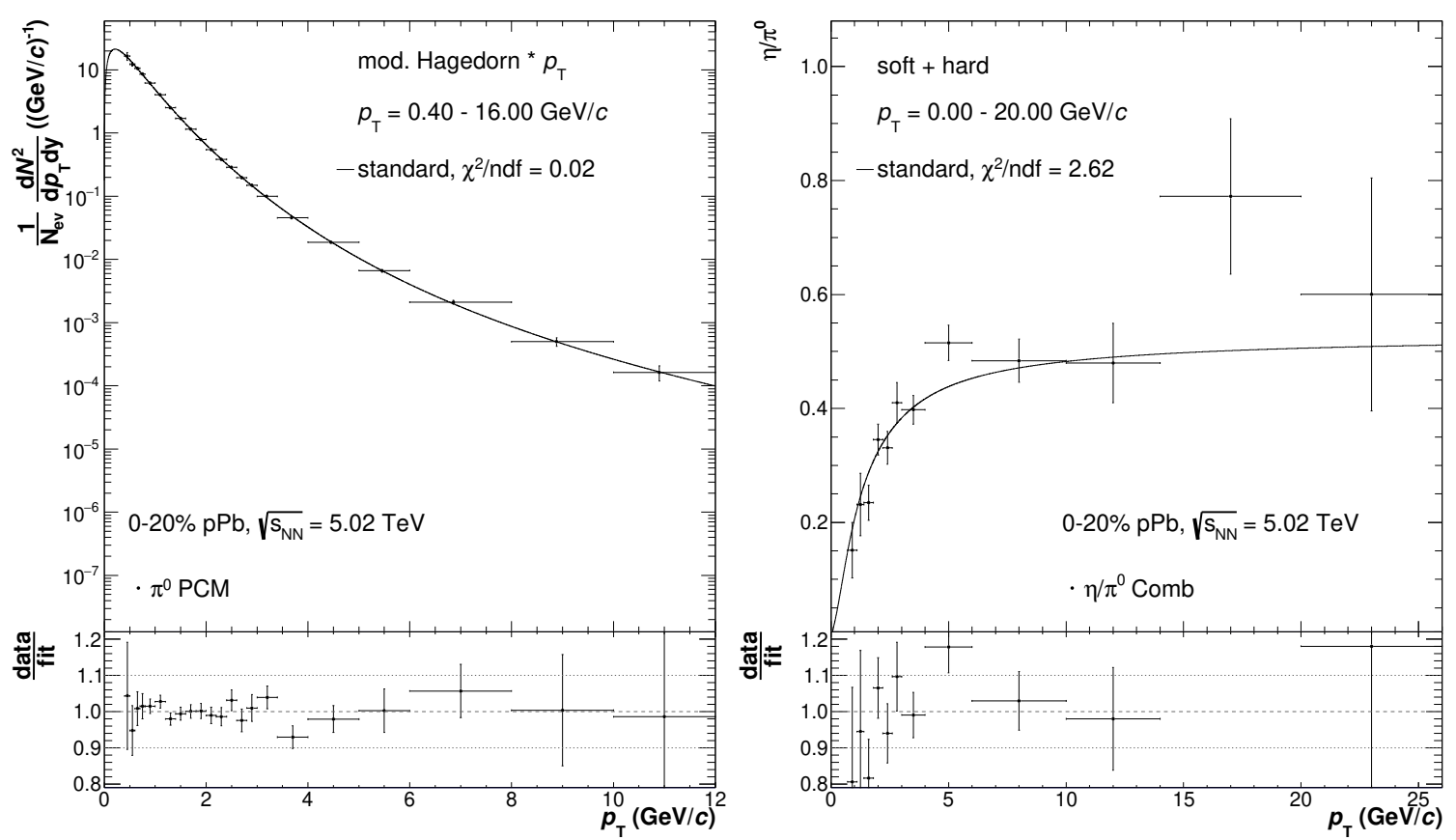

Figure 7.1: Left: Parametrization of the $\pi^{0}$ meson invariant yield measured with PCM in 0-20\% V0A multiplicity in $\mathrm{p}-\mathrm{Pb}$ collisions at $\sqrt{s_{\mathrm{NN}}}=5.02 \mathrm{TeV}$ with a modified Hagedorn function. Bottom panel shows the ratio of data to the fit based on statistical uncertainties. Right: Parametrization of the $\eta / \pi^{0}$ ratio in the same collision system with an empirical function.

The full implementation of the cocktail simulation is available in AliRoot [164] and AliPhysics [165]. It is based on the event generator Pythia6.4 [208] to simulate the full decay chain of randomly generated mother particles flat in transverse momentum $\left(0<p_{\mathrm{T}}<50 \mathrm{GeV} / c\right)$, in the rapidity range $|y|<1.0$ and in the full azimuth $(0 \leq \varphi<2 \pi)$. The resulting mother and daughter particles are weighted with the input parametrizations of the different particle species in order to obtain the correct abundances of each particle. The full list of generated mother particles is given in Table 11 together with relevant information like the rest mass, the considered decay channels as well as the corresponding branching ratios as provided by the particle data group [184]. In addition, for each particle it is denoted if the parametrization was obtained via $m_{\mathrm{T}}$ scaling. If a particle spectrum measurement is available, then a pointer to the reference of the input spectrum is given. The decay photon sample is dominated by contributions from the neutral pion and $\eta$ meson by more than $80 \%$ and 10-15\%, respectively. Further percentlevel contributions come from the $\omega(\approx 2.8 \%)$ and the $\eta^{\prime}(\approx 1.4 \%)$ mesons. The remaining particles listed in Table 11 amount to per-mille level contributions. This puts emphasis on the importance of precise neutral meson measurements, especially for the $\pi^{0}$ and $\eta$ meson. These measurements are described in Ref. [274] and follow the same PCM reconstruction approach as in Section 6.1 for the invariant mass based neutral meson analysis in this thesis for $\mathrm{p}-\mathrm{Pb}$ collisions at $\sqrt{s_{\mathrm{NN}}}=8.16 \mathrm{TeV}$. In addition to the PCM measurement, also other reconstruction techniques using the electromagnetic calorimeters are available [275].

The $\pi^{0}$ meson spectra in each multiplicity class are found to be best described by a modified Hagedorn parametrization. The functional form of this function is introduced in Section 6.1.1.2 and given by Equation 27 in the context of a short introduction of the particle decay simulation as the base ingredient for the secondary $\pi^{0}$ correction. In Figure 7.1 (left) the $\pi^{0}$ meson invariant yield in the $0-20 \%$ multiplicity class is shown together with its modified Hagedorn parametrization as well as the ratio of the data to the fit in the lower panel. The data is very well described over the full $p_{\mathrm{T}}$ range by this functional form within the statistical uncertainties and the function 


\begin{tabular}{|c|c|c|c|c|c|c|}
\hline particle & $\begin{array}{c}\text { mass } \\
\left(\mathrm{MeV} / c^{2}\right)\end{array}$ & $\overline{C_{m_{\mathrm{T}}}}$ & reference & $\begin{array}{c}\text { decay } \\
\text { channels }\end{array}$ & $\begin{array}{l}\text { branching } \\
\text { ratio }\end{array}$ & $\begin{array}{c}\text { contribution } \\
\text { in } \gamma_{\text {dec }} / \gamma_{\text {all }}\end{array}$ \\
\hline$\pi^{0}$ & 134.98 & & this thesis & $\begin{array}{c}\gamma \gamma \\
e^{+} e^{-} \gamma\end{array}$ & $\begin{array}{l}9.882 \mathrm{E}-01 \\
1.174 \mathrm{E}-02\end{array}$ & $>80 \%$ \\
\hline$\eta$ & 547.85 & & this thesis & $\begin{array}{c}\gamma \gamma \\
\pi^{0} \gamma \gamma \\
\pi^{+} \pi^{-} \gamma \\
e^{+} e^{-} \gamma \\
\mu^{+} \mu^{-} \gamma\end{array}$ & $\begin{array}{l}3.941 \mathrm{E}-01 \\
2.560 \mathrm{E}-04 \\
4.220 \mathrm{E}-02 \\
6.899 \mathrm{E}-03 \\
3.090 \mathrm{E}-04\end{array}$ & $10-15 \%$ \\
\hline$\omega$ & 782.65 & $0.85 \pm 0.1$ & $m_{\mathrm{T}}$ scaling from $\pi^{0}$ & $\begin{array}{c}\pi^{0} \gamma \\
\eta \gamma \\
\pi^{0} \pi^{0} \gamma\end{array}$ & $\begin{array}{l}8.350 \mathrm{E}-02 \\
4.600 \mathrm{E}-04 \\
7.000 \mathrm{E}-05\end{array}$ & $\approx 2.8 \%$ \\
\hline$\eta^{\prime}$ & 957.66 & $0.4 \pm 0.2$ & $m_{\mathrm{T}}$ scaling from $\pi^{0}$ & $\begin{array}{c}\rho^{0} \gamma \\
\omega \gamma \\
\gamma \gamma \\
\mu^{+} \mu^{-} \gamma\end{array}$ & $\begin{array}{l}2.908 \mathrm{E}-01 \\
2.746 \mathrm{E}-02 \\
2.198 \mathrm{E}-02 \\
1.080 \mathrm{E}-04\end{array}$ & $\approx 1.4 \%$ \\
\hline$\rho^{0}$ & 775.49 & $1 \pm 0.2$ & $m_{\mathrm{T}}$ scaling from $\pi^{0}$ & $\begin{array}{c}\pi^{+} \pi^{-} \gamma \\
\pi^{0} \gamma \\
\eta \gamma \\
\pi^{0} \pi^{0} \gamma\end{array}$ & $\begin{array}{l}9.900 \mathrm{E}-03 \\
6.000 \mathrm{E}-04 \\
3.000 \mathrm{E}-04 \\
4.500 \mathrm{E}-05\end{array}$ & $<1 \%$ \\
\hline $\begin{array}{l}\rho^{+} \\
\rho^{-}\end{array}$ & 775.49 & $1 \pm 0.2$ & $m_{\mathrm{T}}$ scaling from $\pi^{0}$ & $\begin{array}{l}\pi^{+} \gamma \\
\pi^{-} \gamma\end{array}$ & $\begin{array}{l}4.500 \mathrm{E}-04 \\
4.500 \mathrm{E}-04 \\
\end{array}$ & $<1 \%$ \\
\hline$\phi$ & 1019.46 & - & [271] & $\begin{array}{c}\eta \gamma \\
\pi^{0} \gamma \\
\pi^{+} \pi^{-} \gamma \\
\pi^{0} \pi^{0} \gamma \\
\pi^{0} \eta \gamma \\
\eta^{\prime} \gamma \\
\mu^{+} \mu^{-} \gamma\end{array}$ & $\begin{array}{l}1.310 \mathrm{E}-02 \\
1.273 \mathrm{E}-03 \\
4.100 \mathrm{E}-05 \\
1.130 \mathrm{E}-04 \\
7.300 \mathrm{E}-05 \\
6.300 \mathrm{E}-05 \\
1.400 \mathrm{E}-05\end{array}$ & $<1 \%$ \\
\hline $\begin{array}{l}\Delta^{0} \\
\Delta^{+}\end{array}$ & 1232 & $1 \pm 0.2$ & $m_{\mathrm{T}}$ scaling from $\mathrm{p}$ & $\begin{array}{l}n \gamma \\
p \gamma\end{array}$ & $\begin{array}{l}6.000 \mathrm{E}-03 \\
6.000 \mathrm{E}-03\end{array}$ & $\begin{array}{l}<1 \% \\
<1 \%\end{array}$ \\
\hline$\Sigma^{0}$ & 1192.64 & 0.49 & {$[272]$} & $\Lambda \gamma$ & $1.000 \mathrm{E}+00$ & $<1 \%$ \\
\hline$K_{S}^{0}$ & 497.61 & & {$[57,273]$} & $\begin{array}{c}\pi^{+} \pi^{-} \gamma \\
\pi^{0} \pi^{0}\end{array}$ & $\begin{array}{l}1.787 \mathrm{E}-03 \\
3.065 \mathrm{E}-01\end{array}$ & $<1 \%$ \\
\hline$K_{L}^{0}$ & 497.61 & & {$[57,273]$} & $\begin{array}{c}\pi^{ \pm} e^{\mp} \nu \gamma \\
\pi^{ \pm} \mu^{\mp} \nu \gamma \\
\pi^{+} \pi^{-} \gamma \\
\gamma \gamma \\
\pi^{0} \pi^{0} \pi^{0} \\
\pi^{+} \pi^{-} \pi^{0} \\
\pi^{0} \pi^{0}\end{array}$ & $\begin{array}{l}3.988 \mathrm{E}-03 \\
4.920 \mathrm{E}-04 \\
4.200 \mathrm{E}-05 \\
5.500 \mathrm{E}-04 \\
1.946 \mathrm{E}-01 \\
1.250 \mathrm{E}-01 \\
8.630 \mathrm{E}-04\end{array}$ & $<1 \%$ \\
\hline$\Lambda$ & 1115.68 & & [273] & $\begin{array}{c}n \gamma \\
n \pi^{0}\end{array}$ & $\begin{array}{l}8.400 \mathrm{E}-04 \\
3.580 \mathrm{E}-01\end{array}$ & $<1 \%$ \\
\hline
\end{tabular}

Table 11: Mother particles included in decay simulation. For each particle the respective rest mass in $\mathrm{MeV} / \mathrm{c}^{2}$ is provided as well as the photonic decay channels with their corresponding branching ratios. For particle spectra that are obtained via $m_{\mathrm{T}}$ scaling, the respective $C_{m_{\mathrm{T}}}$ factors including their systematic uncertainty are provided. For measured particle spectra, the corresponding reference to the published or preliminary result is given. 

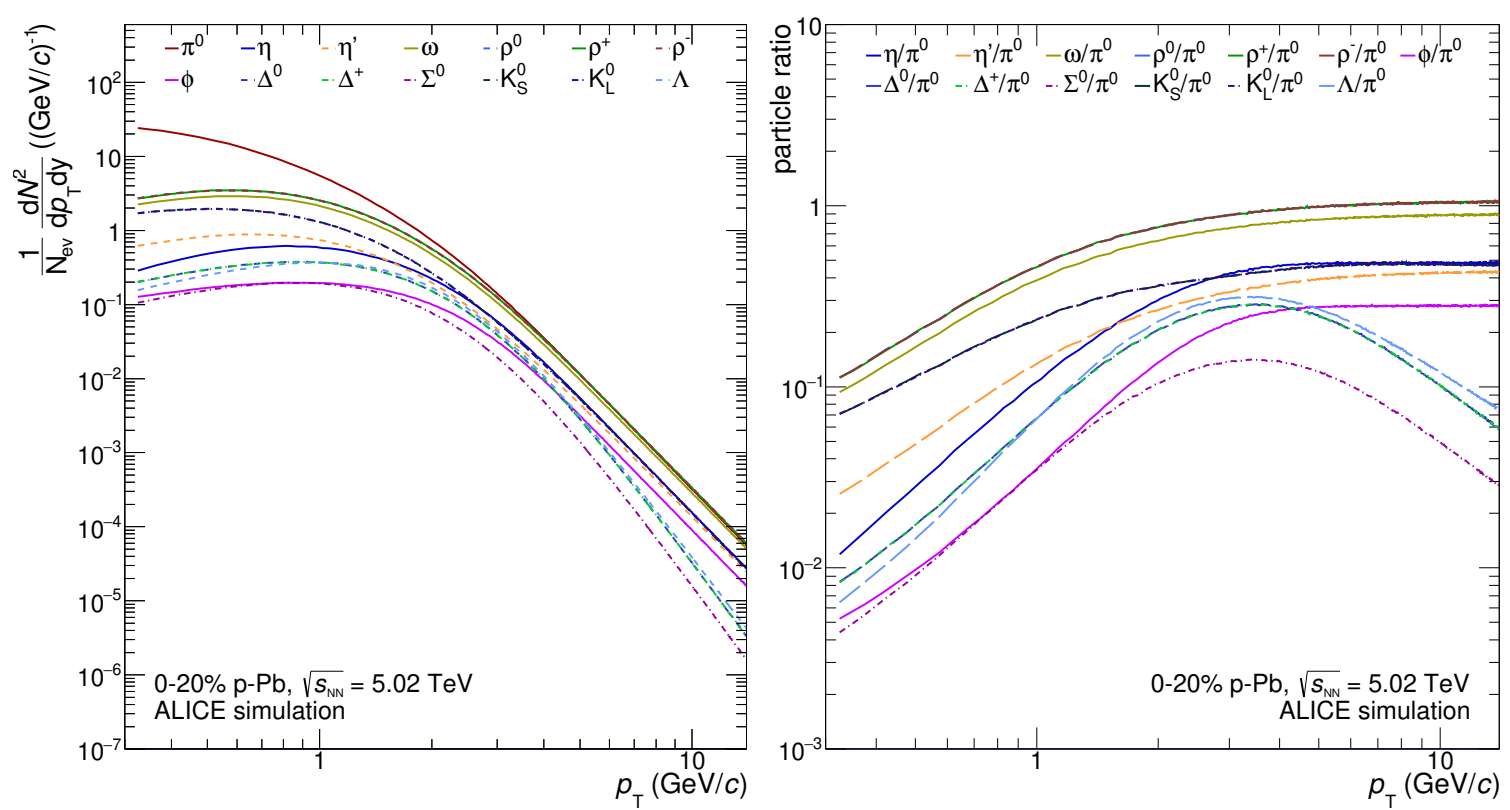

Figure 7.2: Left: Compilation of the cocktail simulation input parametrization for various meson and baryon mother particles in the $0-20 \%$ multiplicity bin in p-Pb collisions at $\sqrt{s_{\mathrm{NN}}}=$ $5.02 \mathrm{TeV}$. Right: Ratios of the spectra from the left panel to the $\pi^{0}$ meson reference spectrum.

is well enough constrained for a reliable extrapolation to higher $p_{\mathrm{T}}$ values, as required for the cocktail simulation up to $p_{\mathrm{T}}=50 \mathrm{GeV} / c$.

The cocktail input parametrization for the $\eta$ meson follows a different approach. Instead of directly fitting the $\eta$ meson spectrum which suffers from comparably large uncertainties and a much more limited $p_{\mathrm{T}}$ reach, the combined $\eta / \pi^{0}$ ratio from all reconstruction techniques is fitted and in a subsequent step this fit is multiplied with the $\pi^{0}$ meson parametrization. This procedure benefits from the high precision of the $\eta / \pi^{0}$ ratio that is achieved by strong cancellations of systematic uncertainties, as well as from the fact that the ratio exhibits a plateau region for $p_{\mathrm{T}}>4 \mathrm{GeV} / c$. This plateau region in the fit ensures that the hard component of the $\pi^{0}$ and the $\eta$ meson spectra is described by the same power and therefore the correct relative abundance of decay products from both mesons is contained in the cocktail. The $\eta / \pi^{0}$ ratio, shown in Figure 7.1, is fitted with an empirical function that contains a blast-wave component [276] to describe the soft part of the spectra and power-law components for the hard parts. The functional form is given by

$$
\frac{\eta}{\pi^{0}}\left(p_{\mathrm{T}}\right)=\frac{A \cdot \exp \left(\frac{\beta p_{\mathrm{T}}-m_{\mathrm{T}}^{\eta}}{T \sqrt{1-\beta^{2}}}\right)+C \cdot R \cdot\left(1+\left(\frac{p_{\mathrm{T}}}{p_{0}}\right)^{2}\right)^{-n}}{\exp \left(\frac{\beta p_{\mathrm{T}}-m_{\mathrm{T}}^{0}}{T \sqrt{1-\beta^{2}}}\right)+R \cdot\left(1+\left(\frac{p_{\mathrm{T}}}{p_{0}}\right)^{2}\right)^{-n}},
$$

where the soft and hard part of the parametrization is normalized via $R$ and the high $p_{\mathrm{T}}$ plateau value is described by $C$. The soft blast-wave component is described via the radial flow velocity $\beta$ and the kinetic freeze-out temperature $T$ which is aimed to describe heavy-ion results but works equally well in small systems. The $\eta / \pi^{0}$ ratio can be described by this functional form within its uncertainties over the full $p_{\mathrm{T}}$ range as shown in Figure 7.1. The remaining contributions for which measurements are available, as listed in Table 11, are parametrized similar to the light neutral mesons with modified Hagedorn parametrizations. 
They include the multiplicity dependent measurements of charged pions and charged kaons [57], neutral kaons and $\Lambda$ $[57,271,273]$ baryons as well as further heavy baryons like $\Omega^{ \pm}, \quad \Sigma^{*+}$ and $\Xi^{ \pm, 0}$ [272]. For particles where no multiplicity dependent measurements are available, $m_{\mathrm{T}}$-scaling $\left(m_{\mathrm{T}}=\sqrt{p_{\mathrm{T}}^{2}+m^{2}}\right)$ is used according to

$$
E \frac{\mathrm{d} N^{\text {part }}}{\mathrm{d} p^{3}}=C_{m_{\mathrm{T}}}^{\text {part }} \cdot f_{\text {ref }}\left(m_{\mathrm{T}}\right),
$$

which allows to obtain a spectrum for a given particle (part) from a reference measurement of another particle (ref). It uses $C_{m_{\mathrm{T}}}$ for the relative scaling of the spectra as given in Table 11 which is obtained from PyтніA or from actual measurements of the particle ratios at high $p_{\mathrm{T}}$. The scaling furthermore requires the use of a meson reference measurement for scaling to another meson and a baryon reference for $m_{\mathrm{T}}$ scaling to another baryon $[227,238]$. It is known that $m_{\mathrm{T}}$-scaling at LHC energies does not hold at low $p_{\mathrm{T}}$ [234] and the scaling relation worsens when using lighter particles as reference which contain large portions of feed-down. This effect, however, is not crucial in this analysis as only min-

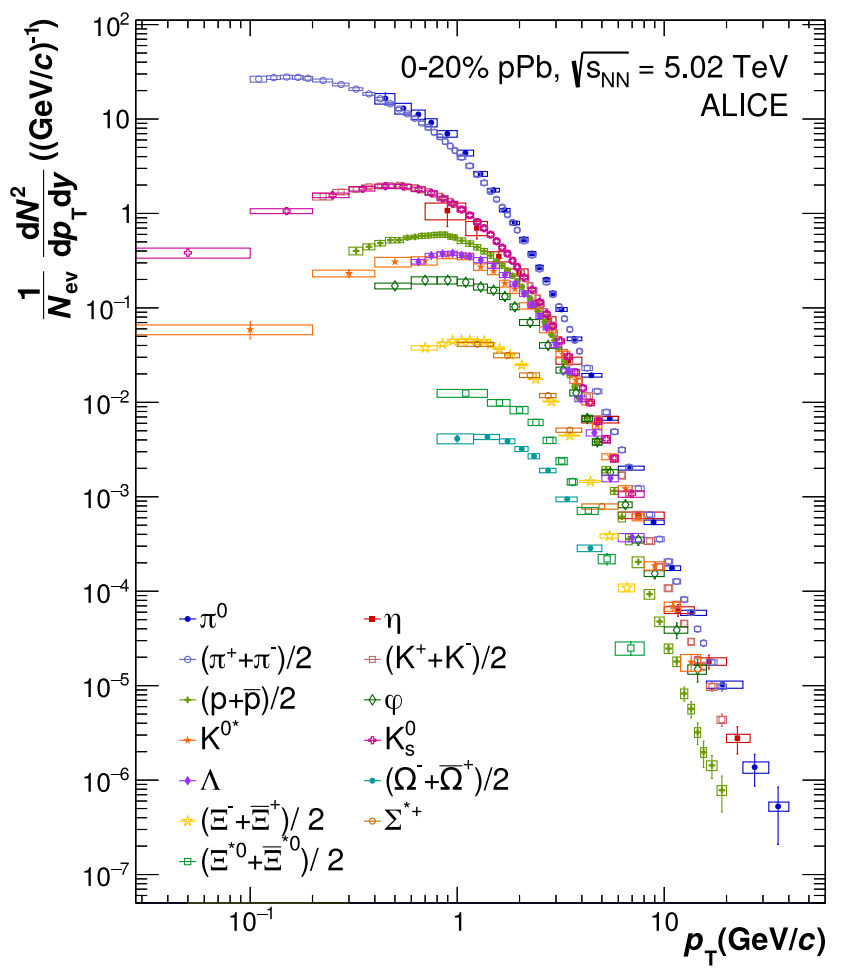

Figure 7.3: Invariant yield of a variety of particle species in the $0-20 \%$ multiplicity bin in $\mathrm{p}-\mathrm{Pb}$ collisions at $\sqrt{s_{\mathrm{NN}}}=5.02 \mathrm{TeV}$. Shown are $\pi^{0}$ and $\eta$ mesons [274], identified charged particles $\left(\mathrm{K}^{ \pm}\right.$, $\left.\mathrm{p}(\overline{\mathrm{p}}), \pi^{ \pm}\right)$[57], neutral kaon, $\varphi$ and $\Lambda$ measurements $[57,271,273]$, as well as heavier baryons $\left(\Omega^{ \pm}\right.$, $\left.\Sigma^{*+}, \Xi^{ \pm, 0}\right)[272]$. imally contributing particles are $m_{\mathrm{T}}$-scaled for the decay simulation. An overview of all particle spectra of mesons and baryons that are used as inputs for the cocktail simulation in the 0-20\% multiplicity class is shown in Figure 7.3. The plot shows the $p_{\mathrm{T}}$ reach of the various measurements as well as statistical and systematic uncertainties represented by vertical bars and boxes, respectively. A compilation of all parametrizations based on these spectra is shown in Figure $7.2(\mathrm{left})$. Presented are parametrizations from direct fits on the invariant yield or parametrizations from $m_{\mathrm{T}}$-scaling. In addition, Figure 7.2 (right) shows the ratios of the various parametrizations to the neutral pion reference spectrum. The ratios also show the plateau region at high $p_{\mathrm{T}}$ for mesons and a significant shape difference for baryons visualizing why baryons can not be $m_{\mathrm{T}}$-scaled from mesons.

The cocktail is then produced based on these input parametrizations and the mother and daughter particles are weighted accordingly. The final decay photon cocktail is then restricted to the acceptance that is used in the analysis of the neutral mesons and the inclusive photons of $|y|<0.8$. Figure 7.4 (left) shows the final decay photon spectra based on all inputs listed in Table 11 while each contribution relative to the total amount of decay photons in the simulation is shown in Figure 7.4 (right). The cocktail is dominated by the contribution from $\pi^{0}$ meson decay photons with more than $80 \%$ while the $\eta$ meson contributes $2-10 \%$ for $p_{\mathrm{T}}<2 \mathrm{GeV} / c$ and up to $16 \%$ at high momentum. The remaining relevant contributions come from the heavier $\omega$ and $\eta^{\prime}$ mesons with $1-2.8 \%$ and $\sim 1 \%$, respectively, as shown in Figure 7.4. For the contributions of the remaining particles, even their summed fraction is well below the current precision of our measurements and therefore they can be considered negligible. 

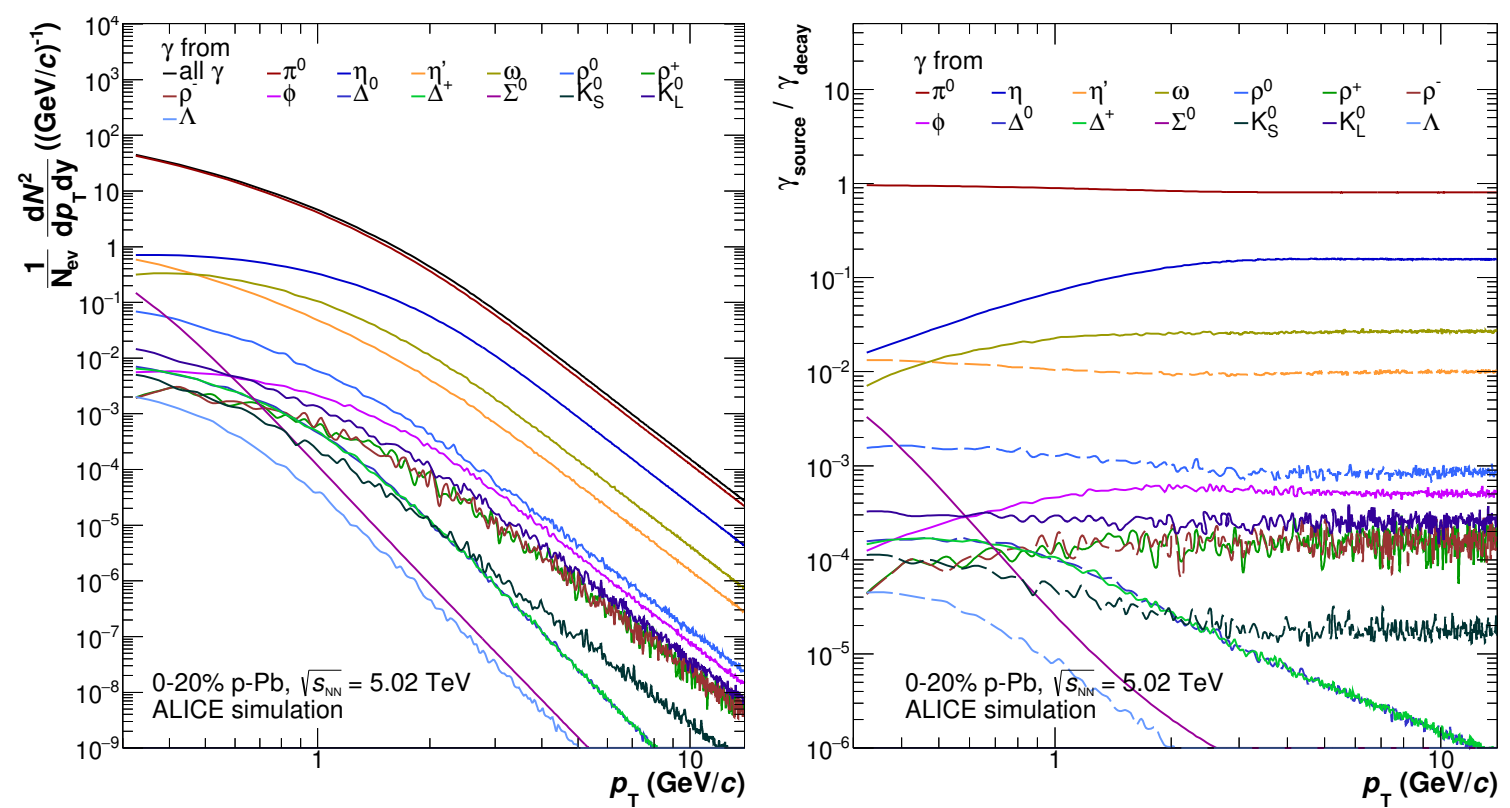

Figure 7.4: Left: Decay photon spectra for 0-20\% V0A multiplicity p-Pb collisions at $\sqrt{s_{\mathrm{NN}}}=$ $5.02 \mathrm{TeV}$ from the cocktail simulation. Right: Decay photon cocktail contributions of various particle species relative to all decay photons.

The full procedure of the decay photon cocktail creation is repeated for all V0A multiplicity classes in the analysis $(0-20 \%, 20-40 \%, 40-60 \%, 60-10 \%)$ and the corresponding plots can be found in the appendix in Section A.9.1.

\subsection{Multiplicity dependent inclusive and direct photon measurements}

The inclusive photon spectra are obtained by applying multiple corrections on the photon candidate raw yield. This $p_{\mathrm{T}}$-dependent raw yield consists of all conversion photon candidates that survive the imposed track and $\mathrm{V}^{0}$ selection criteria. This photon analysis employs marginally different selection criteria with respect to those applied in Section 5 in order to avoid biases compared to the neutral meson analysis from Ref. [232,270]. The necessary correction factors for the inclusive photon analysis are obtained from the same HIJING-based Monte Carlo simulations that are used for the respective neutral meson analyses.

The photon reconstruction with PCM, especially for the low $p_{\mathrm{T}}$ conversion photon candidates, is largely based on exclusive track reconstruction with the TPC. While the SPD is able to reject in-bunch pileup events with a high efficiency, see Section 4.2, the TPC with its long drift times can not easily distinguish tracks from different events. This so-called out-of-bunch pileup increases with the instantaneous luminosity inspected by ALICE and needs a statistical approach for its removal as it can not be determined on an individual photon candidate level. It follows the same approach as in the neutral meson analysis in Section 6.1.1.1 using the distance of closest approach to the primary vertex along the beam axis $\left(d c a_{z}\right)$ of each conversion photon candidate, which shows a Gaussian-like widening of the distribution in a high pile-up environment. In this approach, the candidates are classified into different categories based on their available ITS information. With this information it can be assumed that if both electrons have at least two ITS track points (category 3) then they do not stem from out-of-bunch pileup. However, the dominant fraction of conversion photons contains only TPC information on both electron legs (category 1) and is strongly affected by out-of-bunch pileup. Figure 7.5 (left) shows the $d c a_{z}$ distribution at low $p_{\mathrm{T}}$ for the strongly affected category 1 together with the pileup-free category 3. A clear widening of the TPC-only category is seen compared to category 3 with an additional 

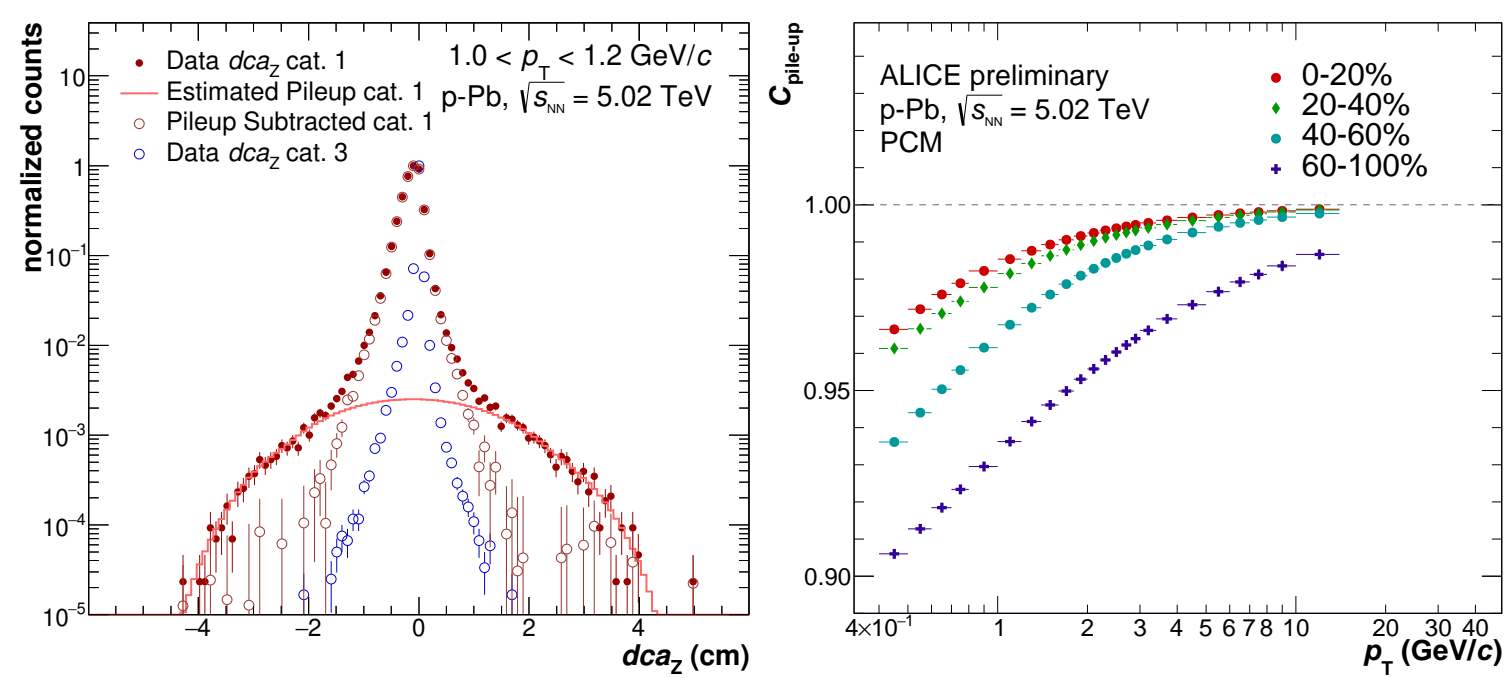

Figure 7.5: Left: Distance of closest approach to the primary vertex along the beam axis $\left(d c a_{z}\right)$ for PCM photon candidates in 0-20\% V0A multiplicity p-Pb collisions at $\sqrt{s_{\mathrm{NN}}}=5.02 \mathrm{TeV}$ at low $p_{\mathrm{T}}$. The TPC-only category 1 is compared to the out-of-bunch pileup free category 3 together with the ShowBackground distribution. Right: Out-of-bunch pileup correction factor $C_{\text {pile-up }}$ for all multiplicity classes.

underlying gaussian-like distribution. The asymmetry in the category 1 distribution is attributed to the $\mathrm{p}-\mathrm{Pb}$ collision system which is boosted in forward direction and thus relies predominantly on different parts of the ITS and TPC. The gaussian-like underlying distribution is attributed to out-of-bunch pileup and described using a Root internal background estimator which is shown as a red line in Figure 7.5. The final correction factor $C_{\text {pileup }}$ is determined from the fraction of background photons in category 1, as obtained from the background estimator, relative to all photons reconstructed in this category. It is shown in Figure 7.5 (right) for all multiplicity slices as a function of transverse momentum. For low multiplicity events, the out-of-bunch pileup correction reaches values of up to $10 \%$ at low $p_{\mathrm{T}}$ while in high multiplicity events it is at most $4 \%$ and negligible for $p_{\mathrm{T}}>10 \mathrm{GeV} / c$. As all conversion photon candidates are required to include TPC tracking information and the resulting $e^{+} e^{-}$pairs are subject to bending in the magnetic field, the bulk of low $p_{\mathrm{T}}$ photon candidates in the analysis originates from late conversions in the outer ITS layer or the TPC frame. These candidates are therefore not constrained by ITS tracking information and are responsible for the increasing correction factor at low $p_{\mathrm{T}}$. The higher the $p_{\mathrm{T}}$, the more tracks can and will include ITS information and thus are constrained to the primary vertex. The multiplicity dependence of the out-of-bunch pileup correction stems from the relative multiplicity of the initial event compared to the out-of-bunch pileup event. This consequently leads to a larger fraction of out-of-bunch pileup photon candidates in low multiplicity events and significantly lower contamination in high multiplicity events.

The inclusive photon measurements also requires the removal of contributions from secondary photons which are dominated by the decay chain of long lived strange particles (e.g. $\mathrm{K}_{\mathrm{S}}^{0} \rightarrow$ $\pi^{0} \pi^{0} \rightarrow 4 \gamma$ ) as well as from material interactions of hadrons. For this correction, the same particle decay simulation is used as described in Section 7.1 and the obtained contributions from the various particles $\left(\mathrm{K}_{\mathrm{S}}^{0}, \mathrm{~K}_{\mathrm{L}}^{0}\right.$ and $\left.\Lambda\right)$ are converted back to raw yield via reconstruction efficiencies and acceptance corrections obtained from full detector Monte Carlo simulations. With this, the secondary photon contamination in the sample can be removed prior to further application of correction factors. Figure 7.6 presents the relative fractions of secondary photon contamination in the raw photon candidate sample for all four multiplicity classes. It can be seen that the 

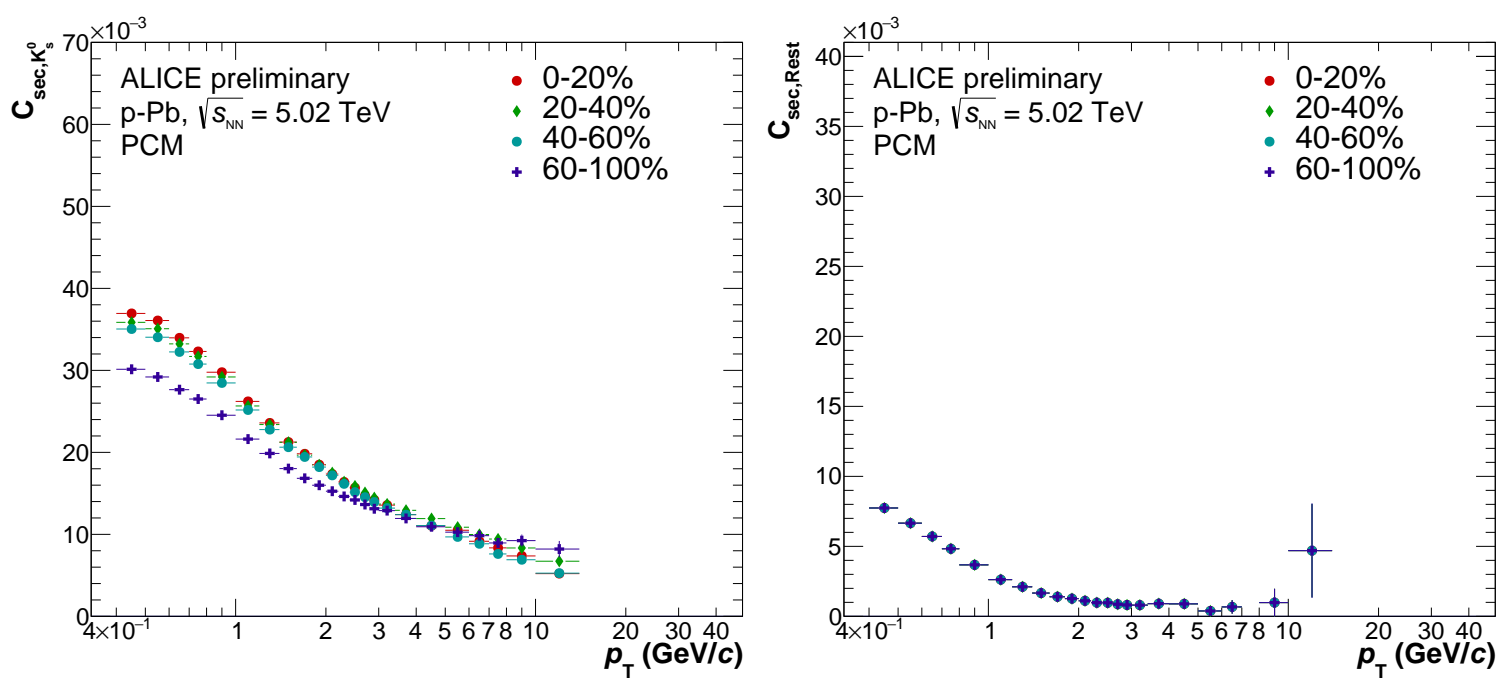

Figure 7.6: Fraction of the secondary photon contamination in the raw photon candidate sample from decays of $\pi^{0}$ mesons coming from $\mathrm{K}_{\mathrm{S}}^{0}$ (left) and coming from interactions of hadrons in the detector material (right) reconstructed with $\mathrm{PCM}$ in four multiplicity bins in $\mathrm{p}-\mathrm{Pb}$ collisions at $\sqrt{s_{\mathrm{NN}}}=5.02 \mathrm{TeV}$.

secondary contamination is approximately independent of multiplicity and ranges from $3.5 \%$ at low $p_{\mathrm{T}}$ to about $1 \%$ at high $p_{\mathrm{T}}$ for the fraction coming from $\mathrm{K}_{\mathrm{S}}^{0}$ decays. Contributions from the other weak decaying particles are less than $1 \%$ while the contribution from material interactions is $\sim 1 \%$ at low $p_{\mathrm{T}}$.

The conversion photon candidate sample, after pileup and secondary contribution removal, contains impurities from unwanted track combinations of the $\mathrm{V}^{0}$ algorithm. This is corrected for with a purity correction, which is defined as the fraction of true reconstructed photons in the photon candidate sample that survived the analysis selection criteria. The purity $\epsilon_{\text {pur }}$, which is determined fully on Monte Carlo simulations, is shown in Figure 7.7 (right) and is approximately the same for all analyzed multiplicity bins. It is around $98-99 \%$ over the full $p_{\mathrm{T}}$ range profiting from the purity-optimized selection criteria in the analysis. The dominant part in the background, as shown in Figure 7.7 (left), are random $e^{+} e^{-}$pairings as well as random track combinations of electrons and charged pions. The latter increase with $p_{\mathrm{T}}$ due to the reduced effectiveness of the TPC $\mathrm{d} E / \mathrm{d} x$ energy loss cuts in the region where the pion and electron signals merge. Further contamination is attributed to random associations of electrons or pions with heavier particles, like charged kaons or protons, but their contribution is close to negligible with less than a per-mille, especially at high $p_{\mathrm{T}}$. This also shows the performance of the $\mathrm{V}^{0}$ selection criteria which suppress such combinations strongly with the applied $\chi^{2}, \Psi_{\text {Pair }}$ and $q_{\mathrm{T}}$ cuts as described in Section 5.

The reconstruction of conversion photons is subject to various detector effects influencing the momentum resolution of the final $\mathrm{V}^{0}$ candidates. These effects are corrected for using Bayesian unfolding [277, 278] which is implemented as part of the Root RooUnfold package [279]. The algorithm is designed to solve the equation

$$
p_{\mathrm{T}, \mathrm{rec}}=\mathcal{A} \cdot p_{\mathrm{T}, \text { true }}
$$

where $\mathcal{A}$ is the response matrix as shown in Figure 7.8 and $p_{\mathrm{T}, \mathrm{rec}}$ is the transverse momentum distribution of the reconstructed photon candidates which is affected by smearing while the initially generated transverse momentum is denoted by $p_{\mathrm{T} \text {,true }}$. The response matrix in Figure 7.8 shows a shift of the reconstructed $p_{\mathrm{T}}$ to smaller values which is attributed to energy loss due to bremsstrahlung. The unfolding procedure with RooUnfold inverts the matrix $\mathcal{A}$ in order to 

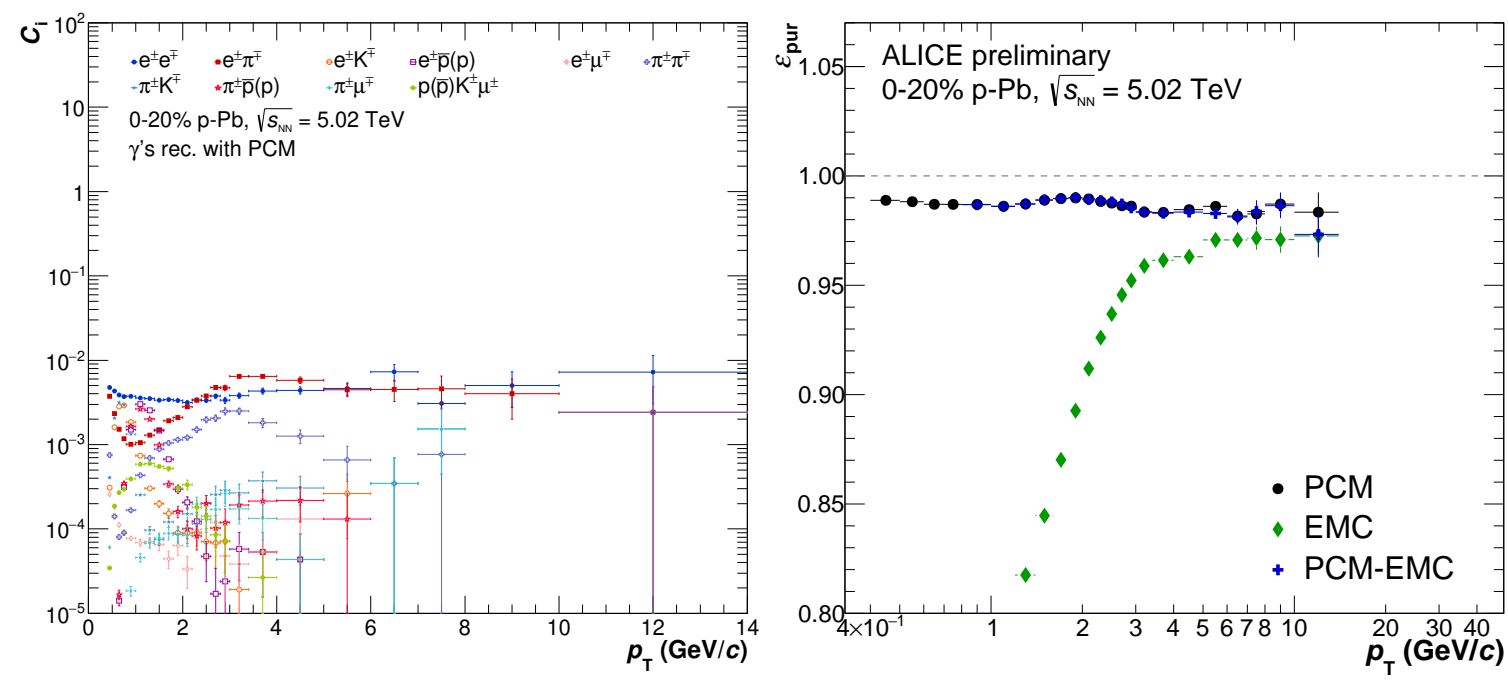

Figure 7.7: Left: Ratio of different identified background sources to the full sample of conversion photon candidates $C_{i}$ versus transverse momentum. Right: Purity of the conversion photon sample reconstructed with $\mathrm{PCM}$ versus $p_{\mathrm{T}}$.

calculate $p_{\mathrm{T}, \text { true }}$. This could, however, experience biases or not be possible at all, depending on the statistical uncertainties of the matrix. An iterative approach using the Bayesan theorem $[277,278]$ is therefore employed which uses as first prior the photon momentum distribution from simulation. Subsequent iterations then employ as prior the unfolded result from the previous iteration until a maximum of four iterations is reached. This procedure is cross checked with the Singular Value Decomposition (SVD) [280] unfolding method that is also part of the RooUnfold package. Both results were found to be consistent on the percent level and thus considered to produce stable results. An additional correction factor needs to be determined, which accounts for all photons that do not convert in the detector material. This conversion probability $\left(P_{\text {conv }}\right)$ is separated from the reconstruction efficiency and entirely based on the true information provided by full detector Monte Carlo simulations.

From the neutral meson analysis it is already known that the material budget is associated with a large systematic uncertainty, which also holds true for the single photon measurements. The conversion probability is shown in Figure 7.9 (left) and is approximately $8 \%$ at very low $p_{\mathrm{T}}$ and approaches a plateau of $8.9 \%$ at high $p_{\mathrm{T}}$.

Separately from the conversion probability, the reconstruction efficiency $\left(\epsilon_{\mathrm{rec}}\right)$ is calculated as the ratio of all validated reconstructed primary photons to all converted photons using Monte Carlo information. The efficiency is shown in Figure 7.9 (right) before the unfolding procedure versus $p_{\mathrm{T}, \text { rec }}$ (open markers) and with the unfolding procedure applied versus $p_{\mathrm{T} \text {,true }}$ (full markers). The unfolded efficiency $\epsilon_{\text {rec }}\left(p_{\mathrm{T}, \text { true }}\right)$ is approximately $15 \%$ larger than $\epsilon_{\text {rec }}\left(p_{\mathrm{T}, \text { rec }}\right)$ starting from intermediate $p_{\mathrm{T}}>2 \mathrm{GeV} / c$ due to radiative energy losses of the electrons. The reconstruction efficiency reaches a maximum value of $71 \%$

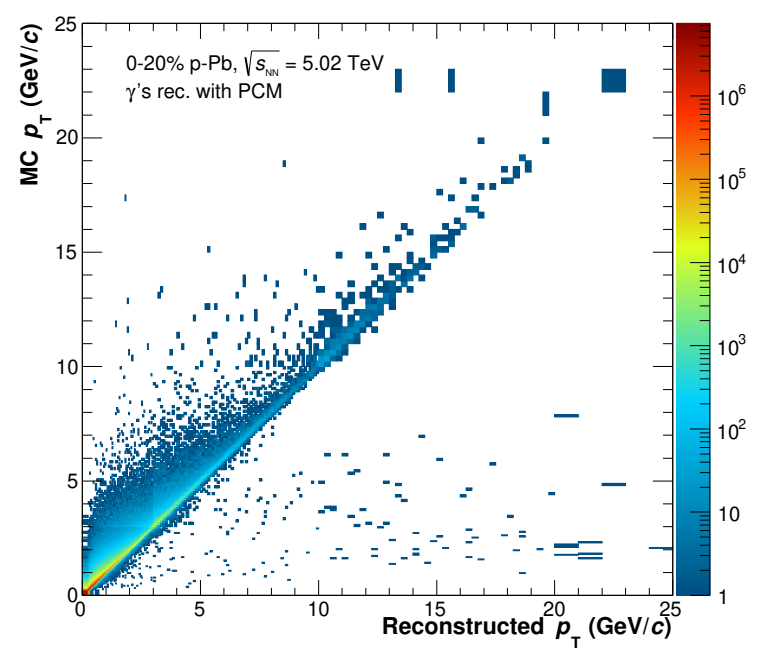

Figure 7.8: Detector response matrix $(\mathcal{A})$ for $\mathrm{PCM}$ photons in $0-20 \%$ multiplicity $\mathrm{p}-\mathrm{Pb}$ collisions at $\sqrt{s_{\mathrm{NN}}}=5.02 \mathrm{TeV}$. 

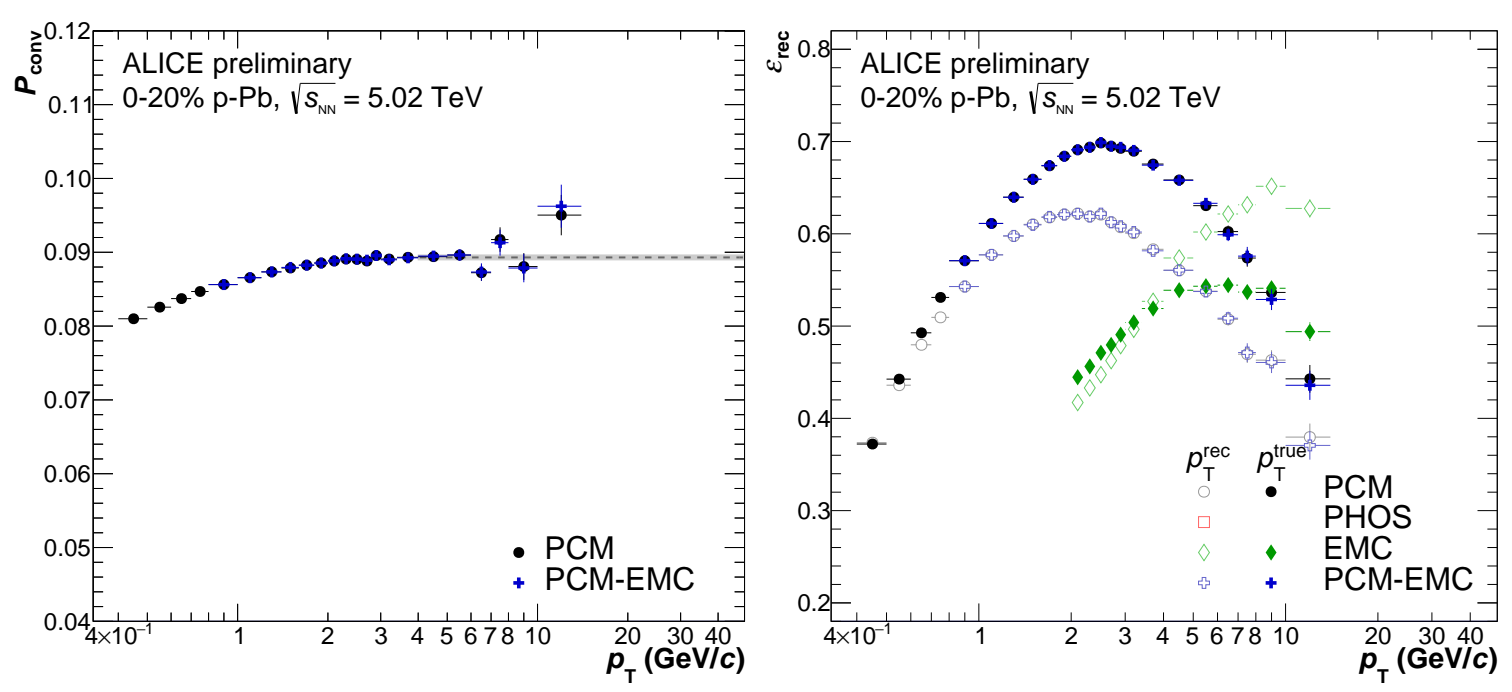

Figure 7.9: Left: Conversion probability $P_{\text {conv }}$ for photons reconstructed with PCM with a high $p_{\mathrm{T}}$ plateau fit of $8.7 \%$. Right: Reconstruction efficiency $\epsilon_{\mathrm{rec}}$ without the unfolding procedure versus $p_{\mathrm{T}, \text { rec }}$ (open markers) and including the unfolding to $p_{\mathrm{T}, \text { true }}$ (full markers).

around $2.5 \mathrm{GeV} / c$ and drops by more than $50 \%$ for very low and very high $p_{\mathrm{T}}$. The low momentum decrease in efficiency is due to the minimum $p_{\mathrm{T}}$ cut on the electrons and the requirement for electrons from conversions to reach the TPC for tracking and PID via $\mathrm{d} E / \mathrm{d} x$. The latter removes large portions of conversion candidates at low radii, where the bending in the magnetic field prevents the resulting $e^{+} e^{-}$pairs to reach the TPC. Furthermore, the PID selection criteria are responsible for the high $p_{\mathrm{T}}$ efficiency loss as the separation of electrons and pions in the TPC energy loss signal worsens and the pion rejection criteria also remove true electron signal.

With the full set of correction factors, the $p_{\mathrm{T}}$-dependent inclusive photon invariant yield can be calculated as

$$
E \frac{\mathrm{d}^{3} N}{\mathrm{~d} p^{3}}=\frac{1}{2 \pi p_{\mathrm{T}}} \frac{1}{N_{\text {evt }}} \frac{\epsilon_{\text {pur }}}{\epsilon_{\text {rec }} \cdot P_{\text {conv }}} \frac{C_{\text {pileup }} \cdot N_{\text {rec,raw }}^{\gamma}-\sum N_{i, \text { sec }}^{\gamma}}{\Delta y \Delta p_{\mathrm{T}}}
$$

where the individual components follow their naming scheme as established in this chapter. With the calculation of the inclusive photon spectrum it is then possible to determine the direct photon excess ratio $R_{\gamma}$ according to

$$
R_{\gamma}=\frac{\gamma_{\mathrm{inc}}}{\gamma_{\mathrm{dec}}} \approx \frac{\left(\gamma_{\mathrm{inc}} / \pi^{0}\right)_{\mathrm{meas}}}{\left(\gamma_{\mathrm{dec}} / \pi_{\mathrm{param}}^{0}\right)_{\mathrm{cocktail}}}
$$

It is originally defined as the ratio of inclusive and decay photons but profits in the double ratio form given in Equation 42 from strong cancellations of uncertainties and the removal of possible biases on the individual components. The calculation of $R_{\gamma}$ requires high precision measurements of the inclusive photons $\gamma_{\text {inc }}$ and the neutral pion spectrum, ideally using the same reconstruction technique. These measurements are compared to the decay photon cocktail $\gamma_{\text {dec }}$ and the simulated $\pi^{0}$ spectrum based on the parametrization of the measured spectrum. Using the precision of the $R_{\gamma}$ measurement, it is possible to determine the direct photon spectrum via the subtraction method as introduced in Equation 9 with $\gamma_{\text {dir }}=\left(1-R_{\gamma}^{-1}\right) \cdot \gamma_{\text {inc }}$. The results of the direct photon analysis will be discussed after an overview of the systematic uncertainties of the measurements and the explanation of the combination procedure for the different reconstruction techniques. 

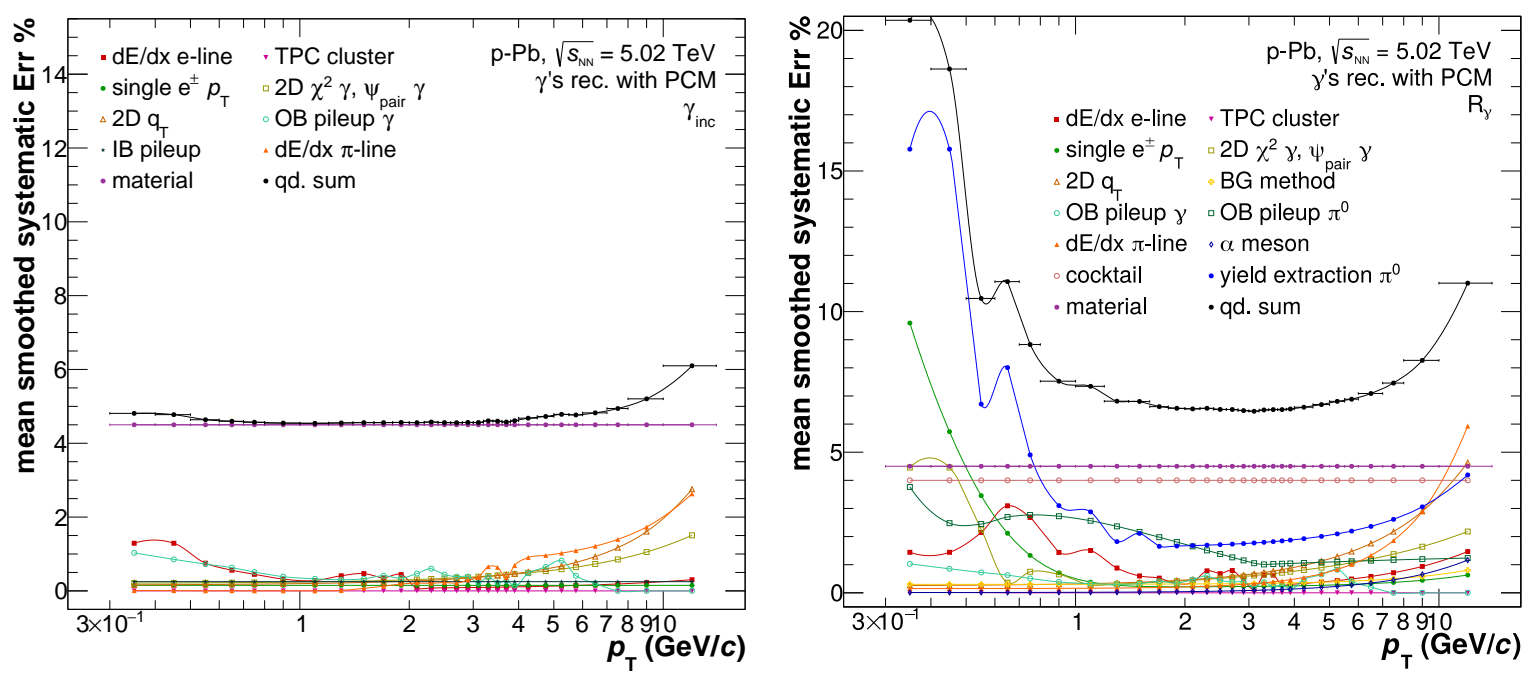

Figure 7.10: Detailed $p_{\mathrm{T}}$-dependence of the individual systematic uncertainty sources for the inclusive photon measurement (left) and the direct photon excess ratio (right). The total uncertainty is indicated in black while individual sources are shown in different colors.

\subsection{Systematic uncertainties}

The systematic uncertainties on the inclusive photon invariant yield and direct photon excess ratio measurements are discussed exclusively for the PCM reconstruction technique in this section for the multiplicity-dependent analysis in $\mathrm{p}-\mathrm{Pb}$ collisions at $\sqrt{s_{\mathrm{NN}}}=5.02 \mathrm{TeV}$. Detailed overviews of the systematic uncertainties for the PCM-EMC, EMC and PHOS reconstruction techniques are given in Ref. [268] and Ref. [269], respectively. The uncertainties are determined in a similar fashion as for the neutral mesons in Section 6.3 with variations of the analysis selection criteria equally on the photon sample and the neutral meson sample to treat correlations in the $R_{\gamma}$ measurement correctly. Influences from statistical fluctuations are reduced by enforcing the Barlow criterium [281] and subsequent smoothing of the obtained uncertainties over three neighboring $p_{\mathrm{T}}$ bins by parameterizing the underlying $p_{\mathrm{T}}$ dependence of each uncertainty source. The $p_{\mathrm{T}}$ dependence of the systematic uncertainties from the various sources is shown in Figure 7.10 for the inclusive photon measurement (left) and the $R_{\gamma}$ measurement (right). In the following, the individual sources shown in Figure 7.10 are explained.

$\mathbf{V}^{0}$ track reconstruction: This category concerns the track quality selection criteria, which are necessary to ensure a good track momentum resolution for the $\mathrm{V}^{0}$ reconstruction. The variations on the minimum track $p_{\mathrm{T}}$ and the TPC clusters are discussed in Section 6.3 and result in significant systematic uncertainties only for the inclusive photon spectrum at low $p_{\mathrm{T}}$ with up to $10 \%$ at $p_{\mathrm{T}}=0.3 \mathrm{GeV} / c$ due to the minimum track $p_{\mathrm{T}}$ cut. For $p_{\mathrm{T}}>1 \mathrm{GeV} / c$ the variations have negligible impact on the inclusive photon and $R_{\gamma}$ measurements with less than $0.2 \%$ associated systematic uncertainty.

Electron identification: The uncertainty associated with the electron selection and charged pion rejection criteria, based on the TPC $\mathrm{d} E / \mathrm{d} x$, are summed in this category. The variations of the selection and rejection criteria are discussed in Section 6.3 and result in an up to $2 \%$ systematic uncertainty on the inclusive photon spectrum at low and high $p_{\mathrm{T}}$ with up to $3 \%$ at low $p_{\mathrm{T}}$ and $5 \%$ at high $p_{\mathrm{T}}$ on the $R_{\gamma}$ measurement. The uncertainty at low momentum is driven by the electron selection criterium in the region where the energy loss signal of the charged pions crosses the electron signal while at high $p_{\mathrm{T}}$ both energy loss signals merge (as seen in Figure 5.4) and the $\pi^{ \pm}$rejection criteria also remove electron signal. 
PCM photon identification: In this category the systematic uncertainties associated with the photon quality selection criteria $\left(\chi^{2},\left|\Psi_{\text {pair }}\right|\right.$ and cuts on the Armenteros-Podolanski distribution) are contained. On the inclusive photon measurement, this uncertainty is $0.5-7 \%$ and increasing with higher $p_{\mathrm{T}}$. On the $R_{\gamma}$ measurement this uncertainty also contributes about $5 \%$ at low $p_{\mathrm{T}}$ as it affects the $\pi^{0}$ measurement differently than the single photons.

Signal extraction of $\boldsymbol{\pi}^{\mathbf{0}}$ : The systematic uncertainty on the $\pi^{0}$ signal extraction is directly taken from the neutral meson analysis itself as it propagates without cancellation to $R_{\gamma}$. Details on the uncertainty itself can be found in Ref. [270]. This is the dominant uncertainty at low $p_{\mathrm{T}}$ with up to $17 \%$ while for $p_{\mathrm{T}}>2 \mathrm{GeV} / c$ the uncertainty is 2 $4 \%$.

Cocktail decay simulation: The particle decay simulation is based on the parametrizations of measured particle spectra. Each of those spectra comes with its own set of systematic uncertainties which need to be accounted for as a systematic uncertainty on the used cocktail simulation. The baseline parametrizations for the cocktail are fitted only on the statistical uncertainties of the spectra in order to constrain the fits. Including the systematic uncertainties in this step could provide too much freedom for the fit parameters and therefore make an extrapolation of the fit to higher $p_{\mathrm{T}}$ unstable and unreliable. Such an extrapolation is necessary for all particle species entering the particle decay simulation as the simulation generates particles up to $p_{\mathrm{T}}=50 \mathrm{GeV} / c$. The systematic uncertainties on the input spectra are therefore accounted for by shifting their central values based on the systematic uncertainties followed by a refit of the modified spectra. This procedure has the biggest impact on the decay photon cocktail for variations on the $\pi^{0}$ and $\eta$ meson spectra. However, both spectra contain large fractions of $p_{\mathrm{T}}$-independent systematic uncertainties mainly related to the material budget which would cancel in the $R_{\gamma}$ calculation. These uncertainties are therefore taken out before the central value shifting procedure is performed. The input spectra are then shifted by a $p_{\mathrm{T}}$-dependent fraction $\left(C\left(p_{\mathrm{T}}\right)\right)$ of their systematic uncertainties.

The corresponding fractions are shown exemplary for the $\pi^{0}$ meson spectrum in Figure 7.11 provid-

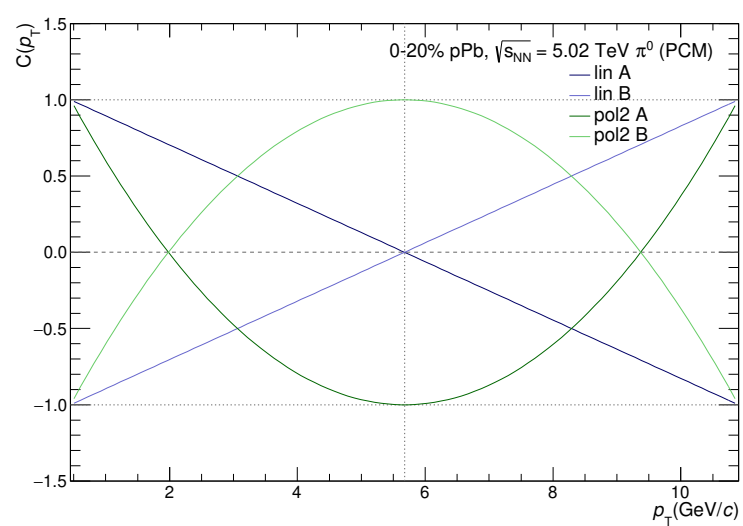

Figure 7.11: Fractional central value shift based on the systematic uncertainty $C\left(p_{\mathrm{T}}\right)$ which is used for all particle species.

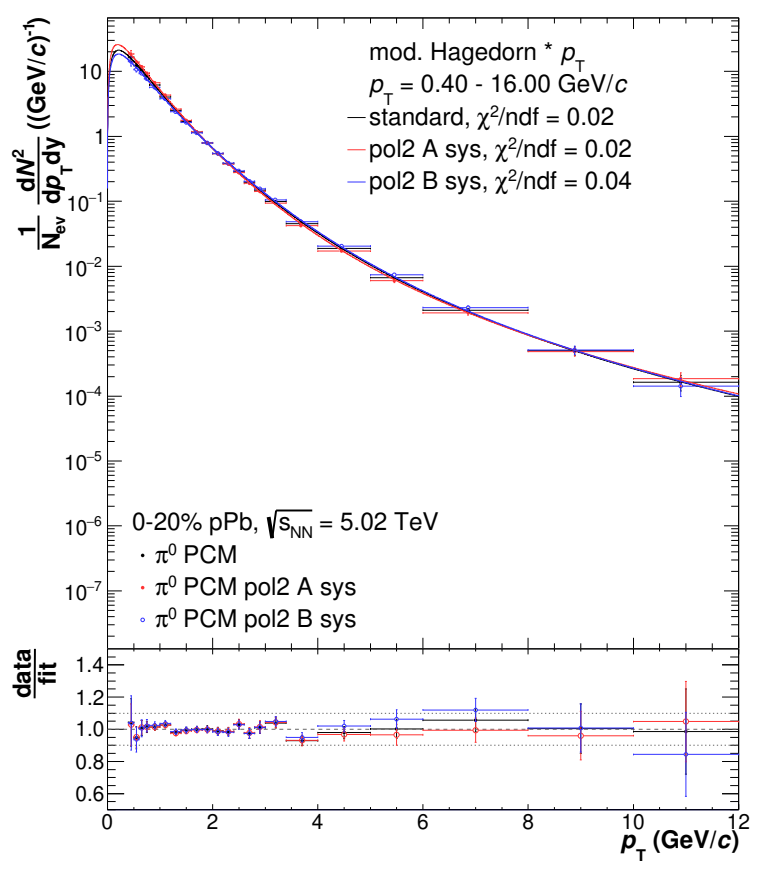

Figure 7.12: Modified $\pi^{0}$ meson spectra with the shifting procedure via $C\left(p_{\mathrm{T}}\right)$ for the two second order polynomial variations (red and blue) with the default spectrum (black). The lower panel presents the ratio of each spectrum to its corresponding fit function. 
ing in general two linear variations which shift the lowest $p_{\mathrm{T}}$ bin either $1 \sigma_{\text {sys }}$ up or down while doing the opposite for the highest $p_{\mathrm{T}}$ bin. In addition, a second order polynomial is used (shown in green) which either has its maximum or minimum at the middle of the $p_{\mathrm{T}}$ range of the spectrum depending on the variation. The result of this shifting procedure is shown exemplary for the $\pi^{0}$ spectrum for the two polynomial variations in Figure 7.12 together with corresponding parametrizations for each variation as well as the ratios between the spectra and the fits.

Based on the new parametrizations for the four systematic shifts, four separate cocktail simulations are produced and used as systematic variations. Aside from the shifted spectra, also the $m_{\mathrm{T}}$ scaling factors are varied in order to account for uncertainties on the $m_{\mathrm{T}}$ scaling factors themselves as listed in Table 11. Based on all variations, the associated systematic uncertainty for the particle decay simulation was determined to be $<0.5 \%$ on the inclusive photon spectrum for the effects on the secondary correction and $p_{\mathrm{T}}$-independent $4 \%$ for $R_{\gamma}$ dominated by the uncertainty of the decay photon sample from the $\pi^{0}$ meson.

Material budget: The dominant systematic uncertainty for photons reconstructed via conversions is based on the limited knowledge of the inner detector material $(5<R<180 \mathrm{~cm})$ and its implementation into Monte Carlo simulations. The material directly affects the conversion probability and its distribution affects the conversion radius and therefore also the reconstruction efficiency. The uncertainty is based on in-depth studies of the material in pp collision data at $\sqrt{s}=7 \mathrm{TeV}[193,212,213]$, which determined a conservative uncertainty estimate of $4.5 \%$ independently of transverse momentum. This uncertainty enters once in the inclusive photon measurement and, after cancellation with the $\pi^{0}$ reconstruction uncertainty, also enters just once in the $R_{\gamma}$ measurement.

Out-of-bunch pile-up: As described in Section 7.2 the PCM measurement is subject to large fractions of out-of-bunch pileup. The associated uncertainty is estimated by variations of the RоOT background estimator on the $d c a_{z}$ distributions. It contains variations on the number of iterations (the magnitude of the background) as well as on the shape of the background. Each variation is chosen to represent a feasible estimate of the background besides the default parameter case. The resulting uncertainty on the inclusive photon measurement is $1.2 \%$ at low $p_{\mathrm{T}}$ and $0.5 \%$ for $p_{\mathrm{T}}>1 \mathrm{GeV} / c$ which translates directly to the $R_{\gamma}$ measurement. An additional component from the $\pi^{0}$ out-of-bunch pileup uncertainty needs to be considered for $R_{\gamma}$ which is $4 \%$ at low $p_{\mathrm{T}}$ and levels out to $1.2 \%$ above $3 \mathrm{GeV} / c$.

Figure 7.13 presents the total statistical and systematic uncertainties for the inclusive photon and $R_{\gamma}$ measurements in 0-20\% V0A multiplicity $\mathrm{p}-\mathrm{Pb}$ collisions at $\sqrt{s_{\mathrm{NN}}}=5.02 \mathrm{TeV}$ for the four available reconstruction techniques. For PCM, the systematic uncertainty on $\gamma_{\text {inc }}$ is approximately $5 \%$ and independent of $p_{\mathrm{T}}$, which is significantly larger than the statistical uncertainty of less than 0.5 per-mille at low $p_{\mathrm{T}}$. The inclusive photon measurement is therefore limited in its precision by the systematic uncertainty, which is largely dominated by the material budget knowledge. For the direct photon excess ratio $R_{\gamma}$ generally larger systematic uncertainties of 6-11\% are estimated, as the additional decay simulation and neutral meson reconstruction uncertainties enter in addition. The statistical precision of the $R_{\gamma}$ measurement is $4-25 \%$ and largely limited by the $\pi^{0}$ meson signal extraction. 

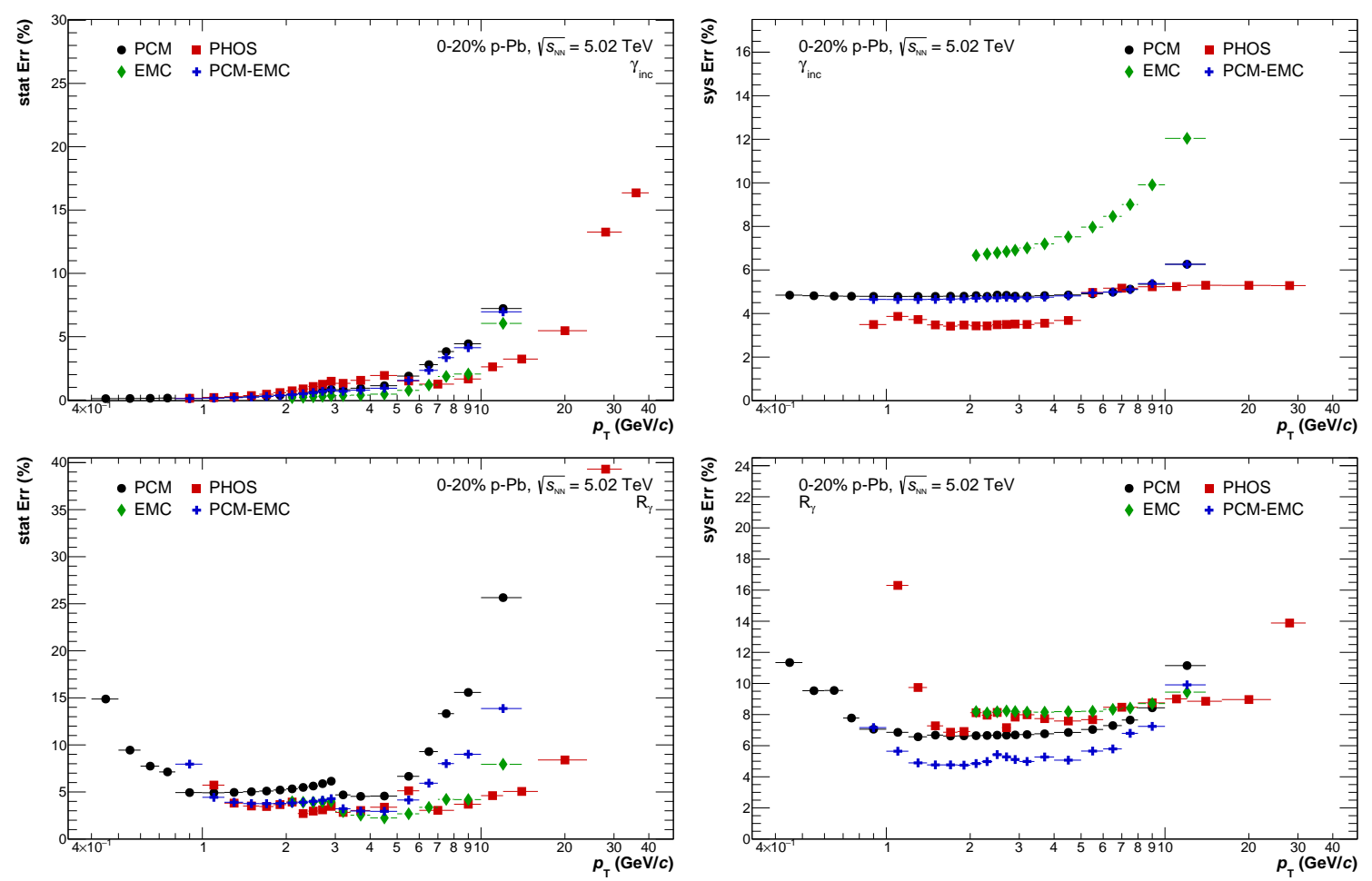

Figure 7.13: Relative statistical (left) and systematic (right) uncertainties in percent for the inclusive photon measurements $(t o p)$ and the direct photon excess ratio (bottom) for the four reconstruction methods used in $0-20 \%$ V0A multiplicity p-Pb collisions at $\sqrt{s_{\mathrm{NN}}}=5.02 \mathrm{TeV}$.

\subsection{Combination of measurements}

In this chapter the individual measurements of the differential invariant inclusive photon yield and the direct photon excess ratios reconstructed with the different reconstruction techniques of PCM, PCM-EMC, EMC and PHOS in p-Pb collisions at $\sqrt{s_{\mathrm{NN}}}=5.02 \mathrm{TeV}$ are combined for each multiplicity class $[268,269]$. The individual measurements are listed in Table 10 together with their respective $p_{\mathrm{T}}$ coverage.

Figure 7.14 (left) shows ratios of the final inclusive photon differential invariant yield to a common TCM fit function for the spectra reconstructed with PCM (black), PCM-EMC (blue), EMC (green) and PHOS (red) in four multiplicity classes in $\mathrm{p}-\mathrm{Pb}$ collisions at $\sqrt{s_{\mathrm{NN}}}=5.02 \mathrm{TeV}$. Similar to the neutral meson results presented in Section 6.6, vertical bars represent the statistical errors, while boxes indicate the systematic uncertainties on each $p_{\mathrm{T}}$ slice. All reconstruction techniques follow a common $p_{\mathrm{T}}$ slicing to allow for direct comparisons and combination of the spectra. The different reconstruction techniques agree with each other within their uncertainties while some tension between the EMC measurement and the other techniques appears for $p_{\mathrm{T}}>7$ $\mathrm{GeV} / c$ in all multiplicity classes.

The final results of the $R_{\gamma}$ measurements with all partially uncorrelated reconstruction techniques are shown in Figure 7.14 (right) for the different multiplicity classes. The PCM and PCM-EMC measurements are consistent with unity for $p_{\mathrm{T}}<7 \mathrm{GeV} / c$, while the PHOS measurements in the same $p_{\mathrm{T}}$ region tend to systematically give higher results, which can still be considered consistent with unity within $2 \sigma$ uncertainties. At high $p_{\mathrm{T}}$, the PCM-EMC and PHOS measurements approach values of $R_{\gamma} \approx 1.2-1.4$, while the EMC measurement is up to $10 \%$ below unity.

The results from the individual reconstruction techniques are combined similarly to the neutral 
meson spectra as described in Section 6.5 in order to obtain a single result which profits from the $p_{\mathrm{T}}$ reach and statistics as well as optimal systematic uncertainties of each reconstruction technique. The combination is performed using the BLUE method $[209,210]$ taking estimated correlations of statistical and systematic uncertainties into account.

While for the neutral meson analyses only systematic uncertainty correlations were present, there are significant statistical uncertainty correlations for the PCM and PCM-EMC measurements of $\gamma_{\text {inc }}$ and $R_{\gamma}$. This can be seen in Figure $7.15(t o p)$ where a correlation of more than $90 \%$ is found independent of $p_{\mathrm{T}}$. This correlation originates from the fact that the PCM-EMC method uses the PCM photons for the inclusive photon measurement and differs only in a few of the photon candidate selection criteria. Furthermore, the PCM, EMC and PHOS reconstruction techniques are considered to be statistically uncorrelated with each other on the inclusive photon measurement. The statistical correlation on $R_{\gamma}$ is overall smaller, as the $\pi^{0}$ meson measurements are considered statistically uncorrelated between all methods and thus only the inclusive photon correlation is propagated as shown in Figure 7.15 (top right).

The correlation factors for the systematic uncertainties are shown in Figure 7.15 (bottom) for the $\gamma_{\text {inc }}$ and the $R_{\gamma}$ measurements. As already for the statistical uncertainties, also the systematic uncertainties on the PCM and PCM-EMC measurements are highly correlated as both measurements effectively probe the same data sample. On $R_{\gamma}$ both reconstruction techniques have
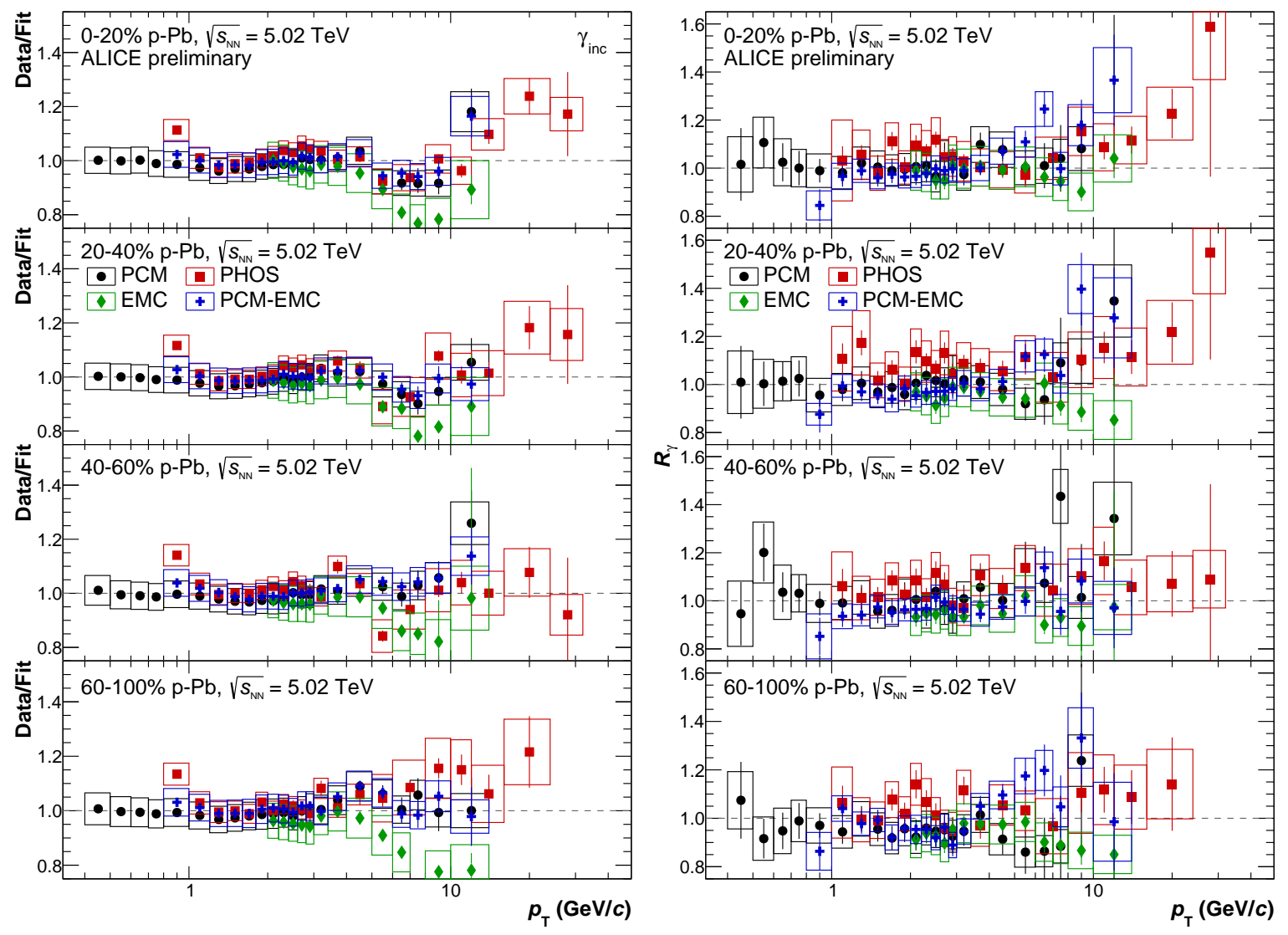

Figure 7.14: Left: Comparison of the individual inclusive photon differential invariant yield spectra from the four different reconstruction techniques to a common fit for all four multiplicity classes. Right: Comparison of the $R_{\gamma}$ measurements for the same reconstruction techniques and multiplicity classes. Vertical bars are statistical uncertainties while boxes indicate the systematic uncertainties. 
correlation factors of $0.2-0.7$ which are reduced by the partially uncorrelated $\pi^{0}$ measurements, where the signal extraction uncertainty is dominant at low and high $p_{\mathrm{T}}$. All reconstruction techniques share a correlated uncertainty due to the particle decay simulation leading to non-zero $C_{i j}$ values.

The combination of the individual $\gamma_{\text {inc }}$ and $R_{\gamma}$ measurements is performed with the BLUE method, using the estimated statistical and systematic uncertainty correlation factors. The resulting weights $\omega_{a}$ according to the BLUE method algorithm can be seen in Figure 7.16 for the 0-20\% V0A multiplicity analysis. The weights for the remaining multiplicity classes can be found in the appendix in Figure A.18. The combination of the inclusive photon spectra is driven by the PHOS measurement with weights of 30-50\% in the overlapping region with other reconstruction techniques. For the first four $p_{\mathrm{T}}$ slices only the PCM measurement is available while for $p_{\mathrm{T}}>10 \mathrm{GeV} / c$ only the PHOS measurement is considered for the combination. In the combination of the $R_{\gamma}$ measurements, the PCM-EMC hybrid method contributes the most with 40-50\% due to its relatively small systematic uncertainties coming from the strong cancellation of material budget uncertainties The total uncertainties of the combined $\gamma_{\text {inc }}$ and $R_{\gamma}$ measurements in the $0-20 \%$ multiplicity class can be found in Figure 7.17 . They are found to be on the order of $2.5 \%$ for the inclusive photon measurements in the intermediate $p_{\mathrm{T}}$ region from 1 to 6 $\mathrm{GeV} / c$ reaching values of $5 \%$ towards lower and higher momenta. The total uncertainties in all multiplicity classes are comparable within $0.3 \%$ and are only subject to the decreasing statistics in low multiplicity events. Similar figures for the other multiplicity classes can be found in the appendix in Figure A.19.

The combined $R_{\gamma}$ measurements present total uncertainties of $4-5 \%$ for $1<p_{\mathrm{T}}<6 \mathrm{GeV} / c$, which are naturally larger than for the inclusive photon measurements due to the additional
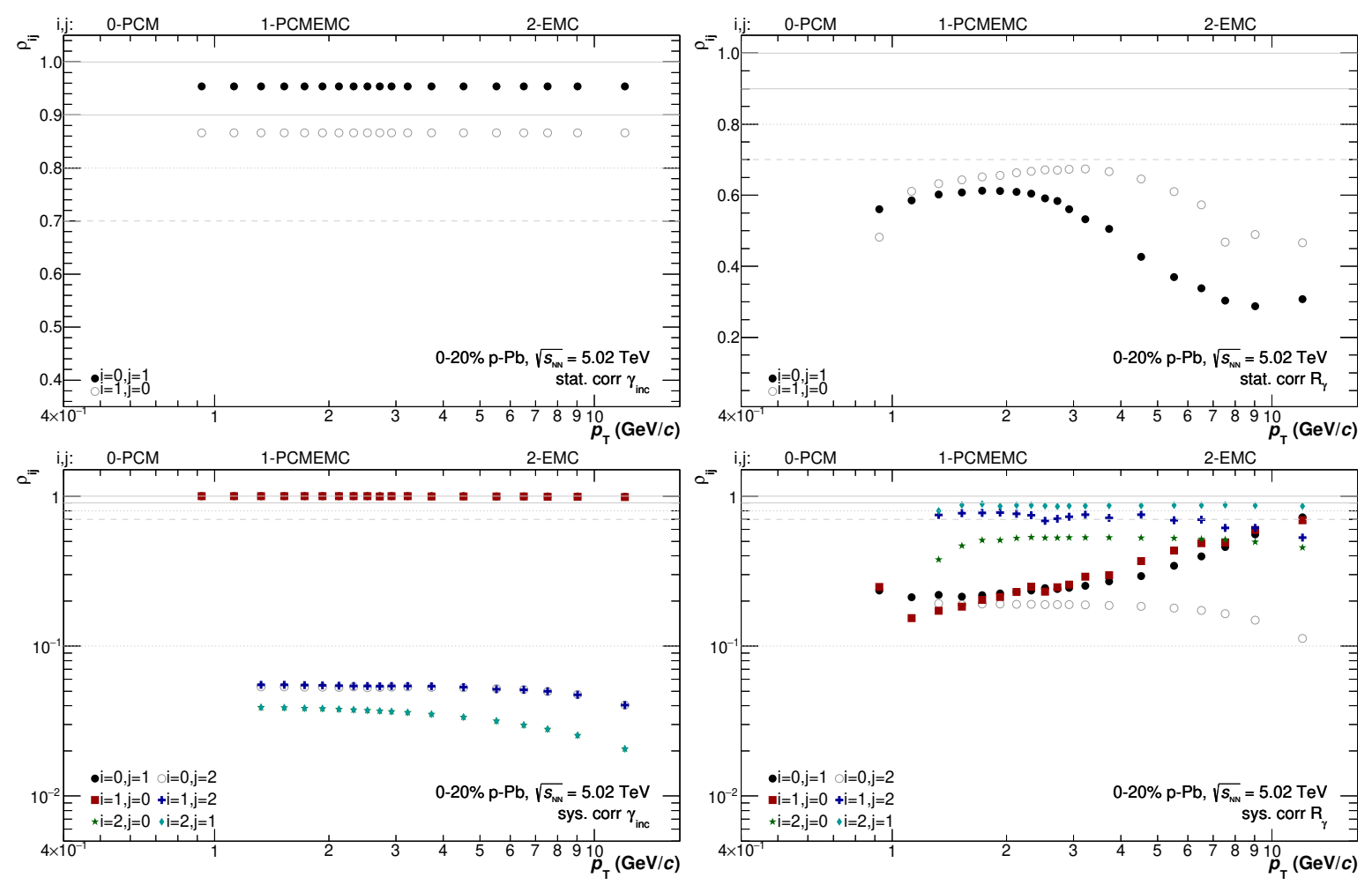

Figure 7.15: Correlation factors $\rho_{i j}$ for the fraction of statistical (top) and systematic (bottom) uncertainty that is correlated between the $\gamma_{\text {inc }}$ (left) and the $R_{\gamma}$ (right) measurements reconstructed with PCM, PCM-EMC and EMC in the 0-20\% V0A multiplicity bin in p-Pb collisions at $\sqrt{s_{\mathrm{NN}}}=5.02 \mathrm{TeV}$. 

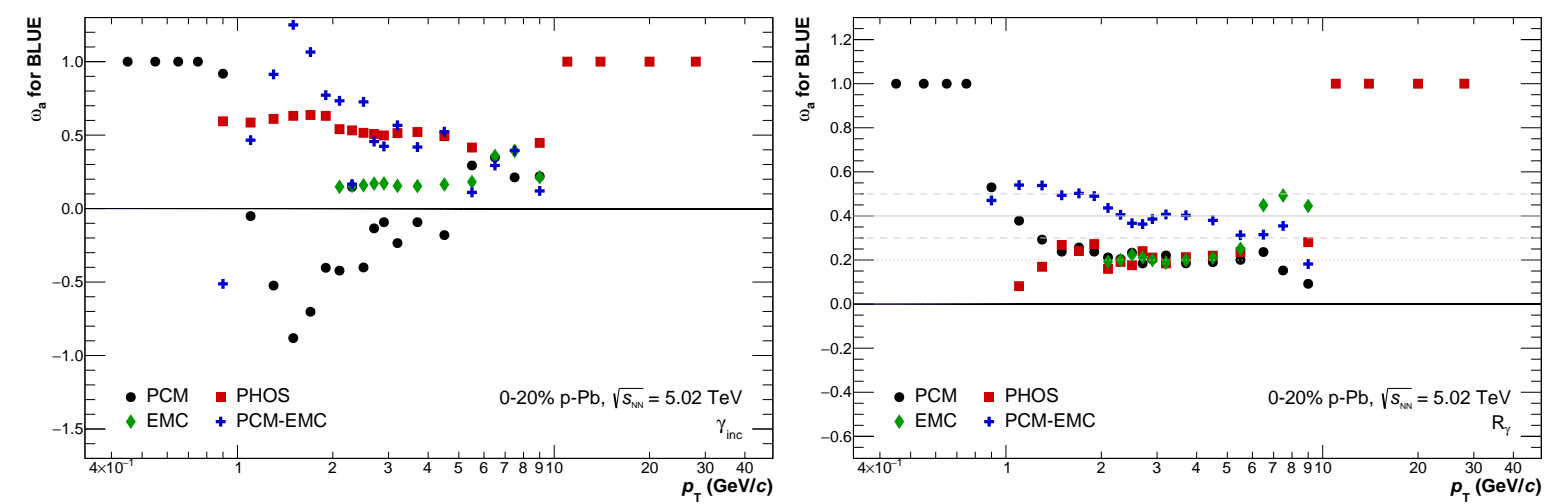

Figure 7.16: Weights $\omega_{a}$ of the individual $\gamma_{\text {inv }}$ and $R_{\gamma}$ measurements according to the BLUE method for the $0-20 \%$ V0A multiplicity bin in $\mathrm{p}-\mathrm{Pb}$ collisions at $\sqrt{s_{\mathrm{NN}}}=5.02 \mathrm{TeV}$.
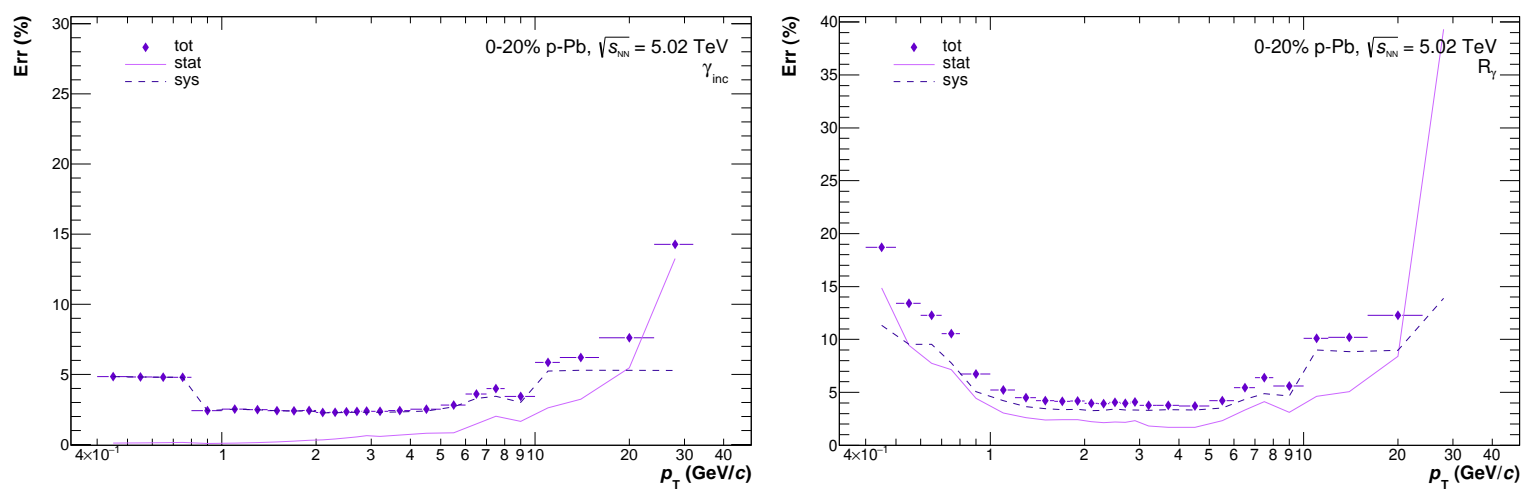

Figure 7.17: Statistical, systematic and resulting total $p_{\mathrm{T}}$-dependent uncertainties of the inclusive photon (left) and $R_{\gamma}$ (right) measurements.

contributions of the particle decay simulation and the $\pi^{0}$ measurement. For $p_{\mathrm{T}}<1 \mathrm{GeV} / c$ and $p_{\mathrm{T}}>6 \mathrm{GeV} / c$ the total uncertainties increase up to $20 \%$, driven by the statistical and systematic uncertainties coming from the $\pi^{0}$ meson signal extraction. Also on the $R_{\gamma}$ measurement, the uncertainties are found to be mostly independent of the multiplicity class and are only affected by decreasing statistics towards lower multiplicities. 


\subsection{Results}
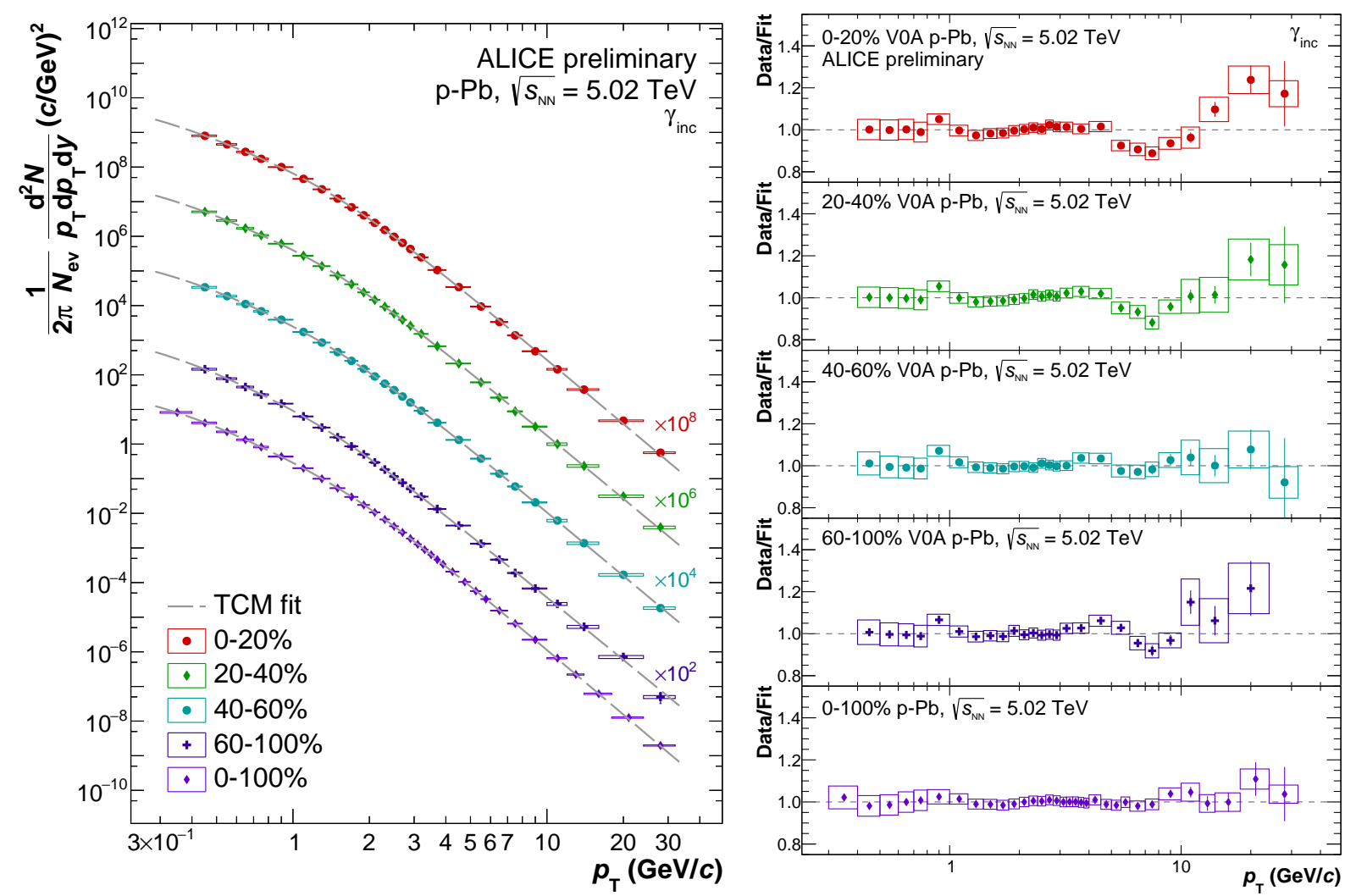

Figure 7.18: Left: Differential invariant inclusive photon yield in four V0A multiplicity classes as well as minimum bias $\mathrm{p}-\mathrm{Pb}$ collisions at $\sqrt{s_{\mathrm{NN}}}=5.02 \mathrm{TeV}$ together with TCM fits. Right: Ratio of the inclusive photon spectra to their TCM parametrization for each multiplicity class.

The inclusive photon invariant yield in $\mathrm{p}-\mathrm{Pb}$ collisions at $\sqrt{s_{\mathrm{NN}}}=5.02 \mathrm{TeV}$ is measured in four V0A multiplicity classes as well as in minimum bias collisions. The final results are obtained by combination of four reconstruction techniques (PCM, PCM-EMC, EMC and PHOS) as described in Section 7.4. The $p_{\mathrm{T}}$-differential spectra, covering a $p_{\mathrm{T}}$ range of $0.4-30 \mathrm{GeV} / c$, are shown in Figure 7.18 (left) for all multiplicity classes including their corresponding two-component model fits according to Equation 34. The parameters of the TCM fits for each multiplicity class are given in Table 12 and show within the uncertainties no hardening or softening of the spectra depending on the multiplicity class. The spectra are scaled with constant factors for better

\begin{tabular}{r|cccccc}
\hline V0A mult. & $A_{e}\left(\mathrm{GeV}^{-2} c^{3}\right)$ & $T_{e}(\mathrm{GeV})$ & $A\left(\mathrm{GeV}^{-2} c^{3}\right)$ & $T(\mathrm{GeV})$ & $n$ & $\chi_{\text {red }}^{2}$ \\
\hline \hline $0-20 \%$ & $260 \pm 820$ & $0.110 \pm 0.090$ & $7.21 \pm 7.24$ & $0.519 \pm 0.110$ & $3.087 \pm 0.012$ & 0.16 \\
$20-40 \%$ & $166 \pm 516$ & $0.110 \pm 0.090$ & $4.61 \pm 5.04$ & $0.509 \pm 0.117$ & $3.048 \pm 0.121$ & 0.11 \\
$40-60 \%$ & $86 \pm 215$ & $0.119 \pm 0.085$ & $2.68 \pm 3.24$ & $0.516 \pm 0.130$ & $3.060 \pm 0.130$ & 0.09 \\
$60-100 \%$ & $54 \pm 187$ & $0.106 \pm 0.092$ & $1.54 \pm 1.99$ & $0.458 \pm 0.121$ & $3.001 \pm 0.121$ & 0.24 \\
$0-100 \%$ & $96 \pm 170$ & $0.126 \pm 0.006$ & $2.51 \pm 2.54$ & $0.544 \pm 0.111$ & $3.109 \pm 0.110$ & 0.20 \\
\hline
\end{tabular}

Table 12: Parameters of the fits to the $\gamma_{\text {inc }}$ invariant differential yield using the TCM fit function $[217,218]$ from Eq. 34 for five multiplicity classes in p-Pb collisions at $\sqrt{s_{\mathrm{NN}}}=5.02$ $\mathrm{TeV}$. 
visualization of the individual $p_{\mathrm{T}}$ reaches and the statistical uncertainties are visualized with vertical bars while systematic uncertainties are given as boxes. Figure 7.18 (right) shows the ratios of the inclusive photon spectra to their respective TCM fits in order to emphasize the descriptive power of the TCM fit function. These fits were also employed for the comparisons of the individual measurements to each other in Figure 7.14. For $p_{\mathrm{T}}<5 \mathrm{GeV} / c$ the fit describes the data in all multiplicity classes perfectly within uncertainties. At higher momentum a 1-2 $\sigma$ discrepancy regarding the systematic uncertainties in the 0-20\% class appears which is driven by the previously observed tension of the EMC measurement with the other reconstruction techniques at high $p_{\mathrm{T}}$. The inclusive photon spectra measurements are in addition used for the calculation of the direct photon excess ratio $R_{\gamma}$, which can be calculated according to Equation 42 as

$$
R_{\gamma} \approx \frac{\left(\gamma_{\mathrm{inc}} / \pi^{0}\right)_{\mathrm{meas}}}{\left(\gamma_{\mathrm{dec}} / \pi_{\text {param }}^{0}\right)_{\mathrm{cocktail}}} .
$$

This approximate calculation of $R_{\gamma}$ profits from bias and uncertainty reductions by adding a normalization via the $\pi^{0}$ spectrum. The necessary $\pi^{0}$ meson spectra have been measured depending on multiplicity in Ref. [270] and the cocktail simulation is described in Section 7.1. With these inputs, $R_{\gamma}$ is calculated in all four multiplicity classes covering $0.4<p_{\mathrm{T}}<30$ $\mathrm{GeV} / c$ as shown in Figure 7.19. In all multiplicity bins, $R_{\gamma}$ is found to be consistent with unity for $p_{\mathrm{T}}<10 \mathrm{GeV} / c$ resulting in no significant direct photon excess within the uncertainties. For $p_{\mathrm{T}}>10 \mathrm{GeV} / c$, the data is consistent with various prompt photon production predictions from NLO pQCD calculations [226] using the CT10 PDF [260], the nCTEQ15 nPDF [77] or the EPPS16 [225] nPDF together with GRV FF [283]. For the comparisons of data to the prompt photon NLO pQCD calculations, the prompt photon $p_{\mathrm{T}}$ spectrum from theory needs to be used as an input for an $R_{\gamma}$ calculation according to

$$
R_{\gamma}^{\mathrm{NLO}}=1+\left(N_{\text {coll }} \cdot \frac{\gamma_{\text {dir }}^{\mathrm{NLO}}}{\gamma_{\text {decay }}^{\text {cocktail }}}\right)
$$

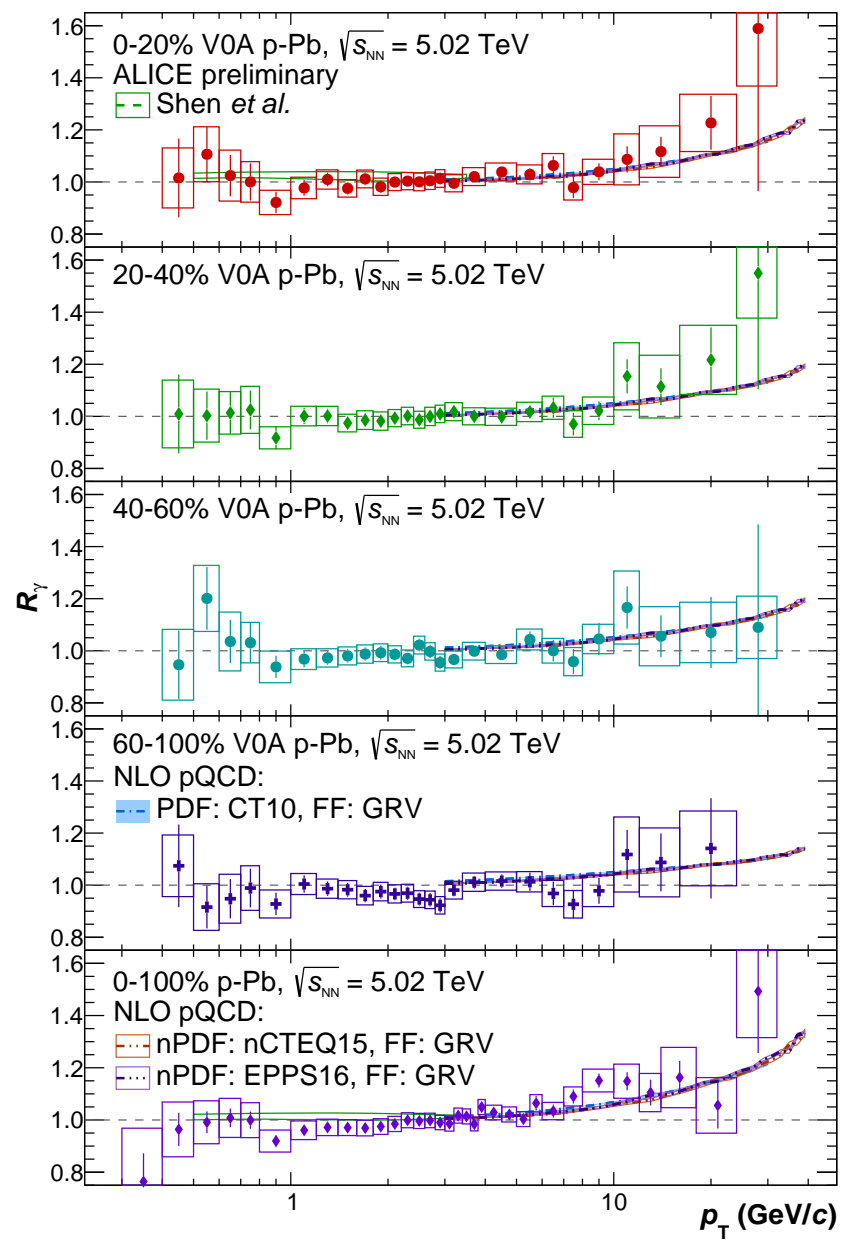

Figure 7.19: Direct photon excess ratio $R_{\gamma}$ in four multiplicity classes as well as in minimum bias p$\mathrm{Pb}$ collisions [88] at $\sqrt{s_{\mathrm{NN}}}=5.02 \mathrm{TeV}$. The data is compared to NLO pQCD calculations $[226,260]$ for prompt photon production at high $p_{\mathrm{T}}$ and compared to hydro calculations by Chun Shen et al. [282] predicting thermal photon production at low $p_{\mathrm{T}}$.

where $\gamma_{\text {decay }}^{\text {cocktail }}$ denotes the decay photon cocktail spectrum and $N_{\text {coll }}$ is given in Table 13 as calculated with Glauber Monte-Carlo simulations [284]. Uncertainties on the NLO pQCD calculations are determined via variations of the factorization, fragmentation and renormalization scales in a range of $0.5 p_{\mathrm{T}}<\mu<2 p_{\mathrm{T}}$. Additional hydro calculations are provided by Chun Shen et. al [282] which predict a direct photon 
excess of $2-3 \%$ in minium bias collisions and $2-5 \%$ in $0-20 \%$ multiplicity for $p_{\mathrm{T}}<3 \mathrm{GeV} / c$. For the minimum bias measurement, an unresolved bias in the analysis resulted in $R_{\gamma}$ central values below unity, but consistent with the predictions and unity within uncertainties [88]. Such a bias is not observed in the 0-20\% multiplicity measurement, however the precision of the measurement does not provide the necessary sensitivity to probe a percent-level direct photon excess.

Using the $R_{\gamma}$ measurements from Figure 7.19 , the direct photon spectra can be calculated using the subtraction method from Equation 9 with $\gamma_{\text {dir }}=\left(1-R_{\gamma}^{-1}\right) \cdot \gamma_{\text {inc }}$. The direct photon spectra are shown in Figure 7.20 for all multiplicity classes and the minimum bias analysis. As the subtraction method requires values of $R_{\gamma}>1$ for the calculation and large portions of the measured data are consistent with unity in $R_{\gamma}$, only upper limits at $90 \%$ confidence level (CL) can be provided in the respective $p_{\mathrm{T}}$ slices. The upper limits are indicated as the vertical bars atop of the arrows in Figure 7.20 and are calculated for each $p_{\mathrm{T}}$ slice where $R_{\gamma}$ is consistent with unity within either $1 \sigma$ of the statistical or the systematic uncertainty. For $p_{\mathrm{T}}$ slices where $R_{\gamma}$ is greater than unity with more than $1 \sigma$ uncertainty, the direct photon spectrum points are calculated and shown as markers with vertical bars as statistical uncertainty and boxes as systematic uncertainty in Figure 7.20. Only for $p_{\mathrm{T}}>10 \mathrm{GeV} / c$ in the highest multiplicity classes and the minimum bias measurement, the direct photon spectrum points instead of upper limits could be determined with the exception of a few lower $p_{\mathrm{T}}$ slices. The data is compared to the same theory calculations that were

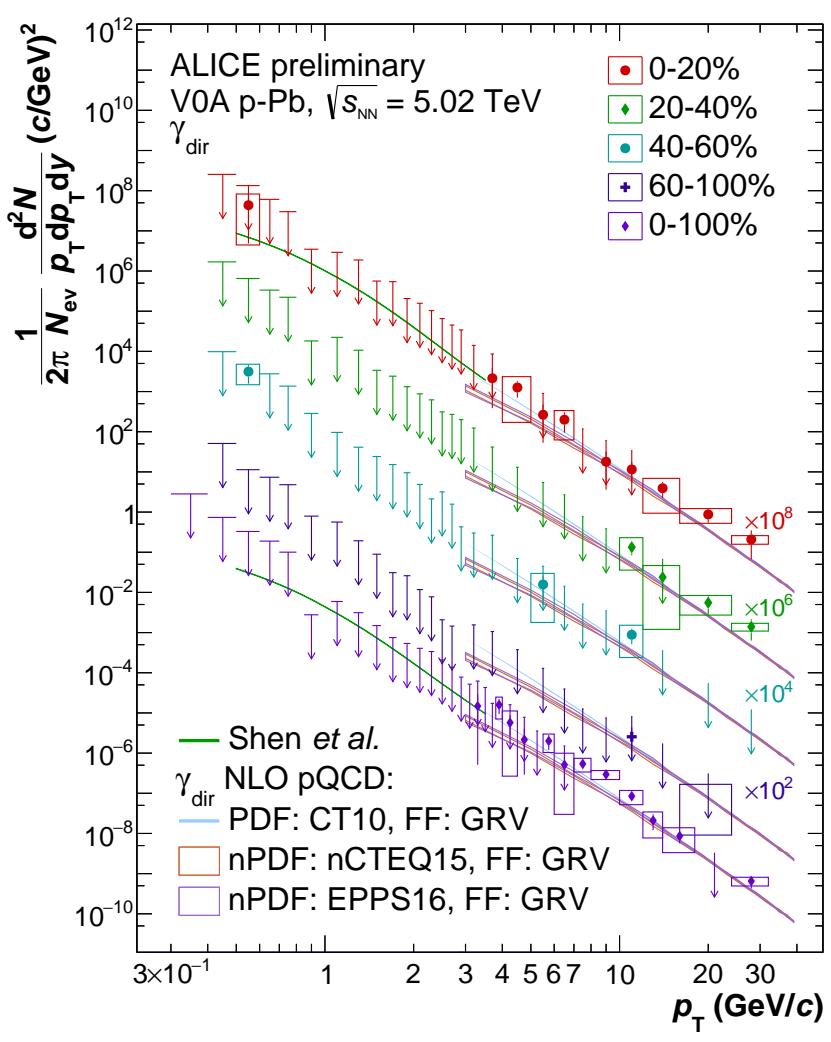

Figure 7.20: Direct photon differential invariant yield (markers) and upper limits (arrows) at 90\% confidence level for four multiplicity classes and minimum bias p-Pb collisions at $\sqrt{s_{\mathrm{NN}}}=5.02 \mathrm{TeV}$. Theory predictions from NLO pQCD and hydro calculations are shown in addition. used in the $R_{\gamma}$ comparisons in Figure 7.19.

The theory is found to be fully consistent with the data points and the upper limits at 90\% CL within uncertainties. These new multiplicity dependent direct photon measurements by ALICE at LHC energies are an important step towards a better understanding of the QGP formation in small collision systems. The data shows no significant excess at low $p_{\mathrm{T}}$, where thermal photon radiation of the hot QGP is expected, in case the medium is created to a sufficient extend. While theoretical calculations predict an excess of $2-5 \%$ in high multiplicity p-Pb collisions, the

\begin{tabular}{cccccc}
\hline & \multicolumn{5}{c}{ V0A centrality class } \\
\cline { 2 - 6 }$N_{\text {coll }}$ & $0-20 \%$ & $20-40 \%$ & $40-60 \%$ & $60-100 \%$ & $0-100 \%$ \\
& $12.23 \pm 0.67$ & $9.09 \pm 0.47$ & $6.42 \pm 0.27$ & $2.93 \pm 0.1$ & $6.71 \pm 0.11$ \\
\hline
\end{tabular}

Table 13: $N_{\text {coll }}$ values from the Glauber Model [284] for various V0A multiplicity classes in p-Pb collisions at $\sqrt{s_{\mathrm{NN}}}=5.02 \mathrm{TeV}$. 
presented measurements show total uncertainties of approximately $5 \%$, thus not providing the required sensitivity to probe such small expected signals.

\subsection{Direct photon summary and outlook}

The first multiplicity dependent measurements of direct photon production in $\mathrm{p}-\mathrm{Pb}$ collisions with ALICE presented in this thesis show no significant thermal photon excess in the $p_{\mathrm{T}}<3$ $\mathrm{GeV} / c$ region within the uncertainties. For this, the highest multiplicity class of $0-20 \%$ was used which corresponds to a charged particle density at mid-rapidity of approximately $\mathrm{d} N_{\mathrm{ch}} /\left.\mathrm{d} \eta\right|_{\eta \approx 0} \approx$ 37 [273]. The multiplicity slicing was largely limited by the available statistics in the $2013 \mathrm{p}-$ $\mathrm{Pb}$ data sample of about 90 million minimum bias events. Additional data was recorded in 2016 adding a factor 5 more statistics to the minimum bias sample. This data was not ready, in terms of calibrations and corresponding Monte Carlo simulations, at the time the presented analysis was performed. Including this data in a re-analysis can therefore greatly improve the statistical precision of the measurements. The additional statistics will provide especially large improvements for the neutral meson measurements which are essential for the calculation of $R_{\gamma}$ and the therein included particle decay simulation. Moreover, during the $2013 \mathrm{p}-\mathrm{Pb}$ data taking campaign, EMCal event triggers were employed, which can provide additional $p_{\mathrm{T}}$ reach and reduced uncertainties up to high transverse momentum as they inspected an approximate factor 150 higher integrated luminosity compared to the minimum bias trigger.

Since the presented direct photon measurement with EMCal has been performed, additional insights into the detector energy response and the shower shape simulation were made [150]. The improved knowledge about the EMCal detector should help eliminate the potential bias in the measurement, as seen in Figure 7.14, and should allow for even more differential measurements. As can be seen in Figure 7.19, the measurements are limited in equal amounts by systematic and statistical uncertainties at low and high $p_{\mathrm{T}}$. There are ongoing studies regarding the material budget systematic uncertainty, which provide weights for MC simulations in order to better reproduce the detector material distribution. Once these studies are completed and final weights are determined, the inner material budget systematic uncertainty could be reduced from the current $4.5 \%$ per conversion photon to approximately $2 \%$. This could enable the PCM and PCM-EMC reconstruction techniques to probe the predicted $2-5 \%$ low $p_{\mathrm{T}}$ direct photon signal with up to $3 \sigma$ significance.

Aside from the $\mathrm{p}-\mathrm{Pb}$ data at $\sqrt{s_{\mathrm{NN}}}=5.02 \mathrm{TeV}$ there is also the data from $\sqrt{s_{\mathrm{NN}}}=8.16 \mathrm{TeV}$ available, which is used in this thesis for the high $p_{\mathrm{T}}$ neutral meson measurements. This data was determined not to be feasible for multiplicity dependent direct photon studies at low $p_{\mathrm{T}}$, as the minimum bias statistics are already only half as much as for the presented direct photon analysis at $\sqrt{s_{\mathrm{NN}}}=5.02 \mathrm{TeV}$. The resulting statistical uncertainties would not allow for a sufficient precision in the $p_{\mathrm{T}}<3 \mathrm{GeV} / c$ region and would in addition only allow to probe a limited $p_{\mathrm{T}}$ region.

Further studies are currently ongoing regarding direct photon measurements in $\mathrm{pp}$ and $\mathrm{Pb}-$ $\mathrm{Pb}$ collision data at $\sqrt{s_{\mathrm{NN}}}=5.02 \mathrm{TeV}$. Such measurements can allow for the calculation of a direct photon $R_{\mathrm{pA}}$ or $R_{\mathrm{AA}}$, where the system and multiplicity dependence of the direct photon production can be further investigated. 


\section{Summary}

Presented in this thesis are the measurements of light neutral mesons in $\mathrm{p}-\mathrm{Pb}$ collisions at $\sqrt{s_{\mathrm{NN}}}=8.16 \mathrm{TeV}$ and in the corresponding reference system of pp collisions at $\sqrt{s}=8 \mathrm{TeV}$ recorded by the ALICE experiment at the CERN LHC in 2016 and 2012, respectively. These measurements aim to constrain nuclear PDFs and to help disentangle initial and final state effects via the nuclear modification factor, which is obtained from comparisons of the particle production in different collision systems. The high center-of-mass energy in the presented analyses provides conditions to test gluon saturation predictions by allowing to probe otherwise hard to reach $x$ and $Q^{2}$ regimes. These measurements are complemented by multiplicity dependent inclusive and direct photon measurements in $\mathrm{p}-\mathrm{Pb}$ collisions at $\sqrt{s_{\mathrm{NN}}}=5.02 \mathrm{TeV}$ aiming to explore a thermal photon signal in small collision systems, which could allow to constrain a possible QGP formation. Photons are reconstructed with two independent techniques employing different detector systems of the ALICE central barrel. The dominant method for low $p_{\mathrm{T}}$ measurements focuses on the reconstruction via $e^{+} e^{-}$pairs from photon conversions in the detector material using tracking and particle identification information from the ITS and the TPC. Complementary measurements are performed with the second method using energy deposits in the electromagnetic calorimeters EMCal and PHOS. The reconstruction of the $\pi^{0}$ and $\eta$ mesons is based on their two-photon decay channel where the signals are either obtained from an invariant mass-based approach or a particle identification method. The invariant mass based approach uses either two photons from the same reconstruction technique, called PCM or EMC (and PHOS), depending on whether both photons are obtained from photon conversions or from EMCal (PHOS) energy deposits, respectively. In addition, the hybrid approach PCM-EMC is employed where one photon from each reconstruction technique is used. This method profits from the high efficiency of the calorimetric photon reconstruction and the high momentum resolution of the conversion photons. For $p_{\mathrm{T}}>16 \mathrm{GeV} / c$, the $\pi^{0}$ meson decay photons can no longer be resolved within the EMCal granularity. Therefore, an independent particle identification approach based on the shower shape of the energy deposit in the EMCal is used to extend the meson measurement to higher momentum. The $p_{\mathrm{T}}$ reach of this reconstruction technique is solely limited by the available statistics in data and the understanding of the correction factors.

With all reconstruction techniques combined, the neutral pion differential invariant cross section in pp collisions at $\sqrt{s}=8 \mathrm{TeV}$ could be measured over a transverse momentum range of $0.3<p_{\mathrm{T}}<200 \mathrm{GeV} / c$ extending a previous measurement by nearly an order of magnitude [196]. A similar transverse momentum reach has been achieved in $\mathrm{p}-\mathrm{Pb}$ collisions at $\sqrt{s_{\mathrm{NN}}}=8.16 \mathrm{TeV}$ for the $\pi^{0}$ meson with the only difference being a more limited low momentum reach to $p_{\mathrm{T}}>0.4$ $\mathrm{GeV} / c$. Both measurements, shown in Figure 6.29, therefore reach the highest transverse momentum for identified particle spectra to date, thus highlighting the strength of the merged cluster analysis. The $\eta$ meson differential invariant cross section spectrum in the pp data sample is extracted covering $0.5<p_{\mathrm{T}}<45 \mathrm{GeV} / c$ and in the $\mathrm{p}-\mathrm{Pb}$ sample covering $1.0<p_{\mathrm{T}}<50$ $\mathrm{GeV} / c$. In both collision systems, the spectra are found to be overestimated by up to $50 \%$ in the central values of next-to-leading order $\mathrm{pQCD}$ calculations based on current parton distribution and fragmentation functions. Especially for the $\eta$ meson, the spectra are largely overestimated as no fragmentation functions including LHC data constraints are available yet. From the spectra, the $\eta / \pi^{0}$ ratios in each collision system are determined and found to have consistent plateau region values for $p_{\mathrm{T}}>4 \mathrm{GeV} / c$ between both systems as shown in Figure 6.30. In addition, the measurements are used to further constrain the global fit value of the $\eta / \pi^{0}$ ratio using inputs from a variety of collision energies and experiments resulting in a decrease of the fit uncertainty. The ratio is also compared to theory predictions which marginally underestimate the data. In 
addition, the $m_{\mathrm{T}}$ scaling prediction is tested and found to be broken at low momentum as seen in previous measurements at LHC energies.

The measured spectra from both collision systems are compared to each other by means of the nuclear modification factor $R_{\mathrm{pA}}$ and a suppression at low $p_{\mathrm{T}}$ is found, compatible with theory predictions based on the CGC model, energy loss in cold nuclear matter calculations as well as next-to-leading order pQCD as shown in Figure 6.34. From comparisons to other measurements at a lower center-of-mass energy, a hint at a stronger suppression at the higher energy system by up to $7 \%$ is observed in the intermediate $p_{\mathrm{T}}$ region. At high momentum, the $R_{\mathrm{pA}}$ measurement is found to be compatible with unity within uncertainties, which stands in possible tension with a charged hadron measurement by CMS that observed a slight enhancement at high $p_{\mathrm{T}}$. The measurement of $R_{\mathrm{pA}}$ can therefore not confirm the presence of a QGP in small systems but given the remaining uncertainties a small droplet can also not be excluded. Further measurements, like $Q_{\mathrm{pA}}$ in high multiplicity $\mathrm{p}-\mathrm{Pb}$ collisions up to high momentum might provide further constrains.

A further effort to observe signatures of the QGP is made with multiplicity dependent measurements of inclusive and direct photons in $\mathrm{p}-\mathrm{Pb}$ collisions at $\sqrt{s_{\mathrm{NN}}}=5.02 \mathrm{TeV}$. With predicted thermal photon signals at low $p_{\mathrm{T}}$ on the order of $2-4 \%$ in high multiplicity collisions, the measured $R_{\gamma}$ could not determine a significant excess, given the $\sim 3-15 \%$ total uncertainty on the measurement as shown in Figure 7.19. However, at high momentum $\left(p_{\mathrm{T}}>10 \mathrm{GeV} / c\right)$ a prompt photon signal consistent with theory predictions of up to $20 \%$ is found. The resulting direct photon spectra are determined wherever $R_{\gamma}$ exceeds unity while for the remaining transverse momentum slices upper limits at $90 \%$ confidence level are provided. The measurement is found to be limited by the statistical precision as well as systematic uncertainties and will profit from increased statistics, which are given in a new data sample from 2016 with a factor 5 more recorded minimum bias events. With this, also finer multiplicity slices of $0-2 \%$ or $0-5 \%$ could be explored to increase the expected relative thermal photon signal and thus allow for a significant measurement within the given uncertainties.

In summary, this thesis has presented identified particle spectra to the highest ever measured transverse momentum and provided a first measurement of multiplicity dependent direct photon production at low momentum in $\mathrm{p}-\mathrm{Pb}$ collisions. The measurements will allow for better constrains of nuclear PDFs as well as energy loss calculations and can serve as a baseline for even more differential analyses. 


\section{A Appendix}

\section{A.1 Overview of data samples and MC productions}

\begin{tabular}{llll}
\hline System & Data periods & MC productions & \\
\hline $\mathrm{pp}, \sqrt{s}=8 \mathrm{TeV}$ & LHC12[a-d,f,h,i] $]$ & LHC15h2[a-d,f,h,i] & Phojet \\
& & LHC15h1[a1-d,f,h,i] & PYTHIA8 \\
& & LHC16c2[_plus] & PYTHIA8 Jet-Jet \\
& & LHC17g5b $\left(p_{\mathrm{T}}^{\gamma}>3.5 \mathrm{GeV} / c\right)$ & PYTHIA8 Jet-Jet $\gamma_{\text {dec }}$ triggered \\
& & LHC17g5c $\left(p_{\mathrm{T}}^{\gamma}>7 \mathrm{GeV} / c\right)$ & in EMCal acceptance \\
\hline $\mathrm{p}-\mathrm{Pb}, \sqrt{s_{\mathrm{NN}}}=5.02 \mathrm{TeV}$ & LHC13[b,c] & LHC13b2 & DPMJET \\
& & LHC13e7 & HIJING with added $\pi^{0}$ and $\eta$ \\
\hline $\mathrm{p}-\mathrm{Pb}, \sqrt{s_{\mathrm{NN}}}=8.16 \mathrm{TeV}$ & LHC16[r,s] & LHC18f3[b,c] & DPMJET \\
& & LHC18b9[b,c] & EPOS LHC with PyTHIA8 jets \\
& & LHC17g6b2[a,b] $\left(p_{\mathrm{T}}^{\gamma}>3.5 \mathrm{GeV} / c\right)$ & PYTHIA8 Jet-Jet $\gamma_{\text {dec triggered }}$ \\
& & LHC17g6b3[a,b] $\left(p_{\mathrm{T}}^{\gamma}>7 \mathrm{GeV} / c\right)$ & in EMCal/DCal acceptance \\
\hline
\end{tabular}

Table 14: Overview of the ALICE data samples and Monte Carlo simulations used in the $\mathrm{pp}$ analysis at $\sqrt{s}=8 \mathrm{TeV}$ and the $\mathrm{p}-\mathrm{Pb}$ analyses at $\sqrt{s_{\mathrm{NN}}}=5.02$ and $8.16 \mathrm{TeV}$. Additional generator information is provided for the simulations. The detector response is simulated in all MC productions with GEANT3.

\section{A.2 Example invariant mass distributions for pp $\sqrt{s}=8 \mathrm{TeV}$}
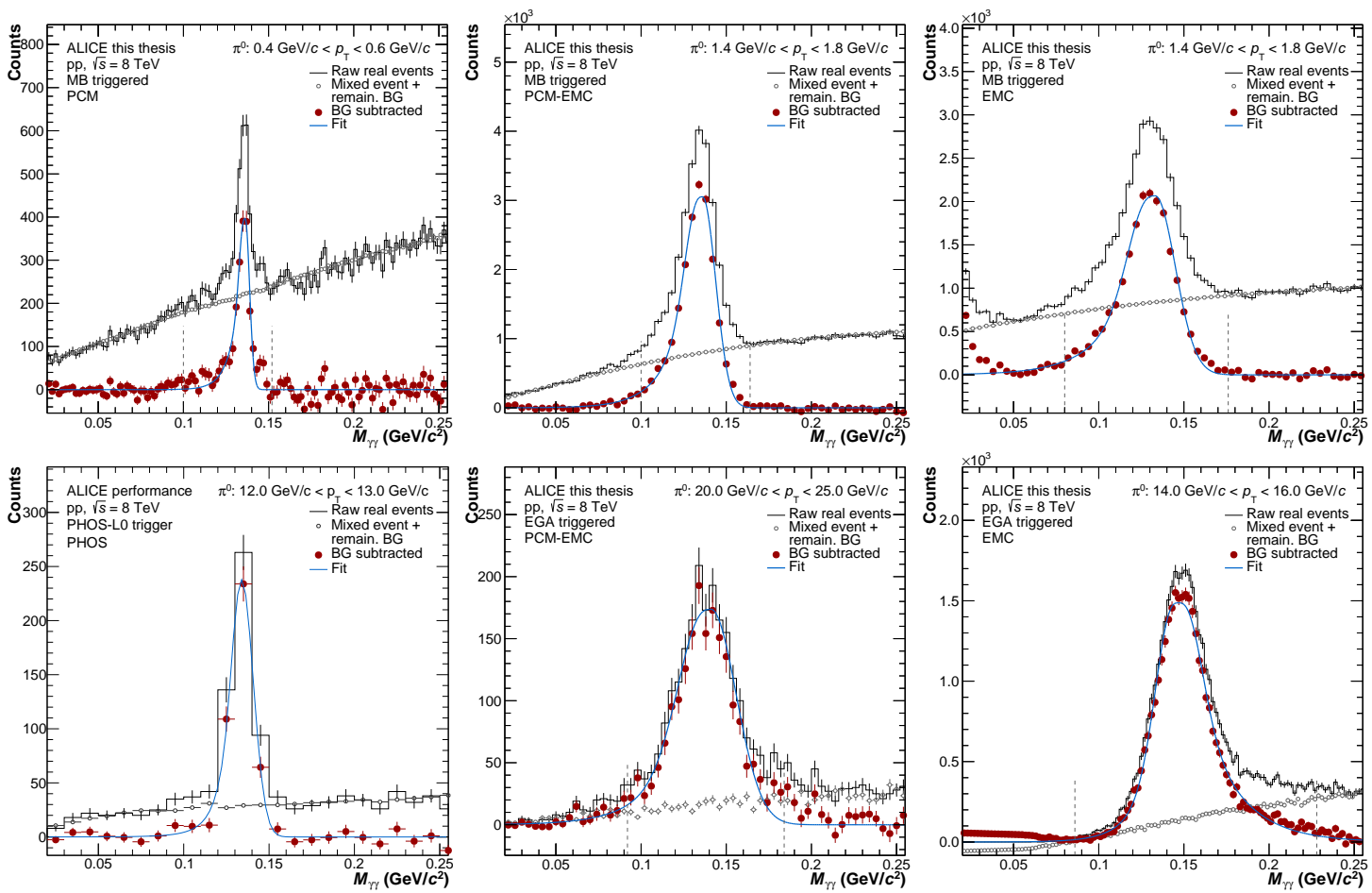

Figure A.1: Invariant mass distributions in a wide window around the nominal $\pi^{0}$ meson mass for pp collisions at $\sqrt{s}=8 \mathrm{TeV}$ minimum bias data (top) and triggered data (bottom) for PCM (left), PCM-EMC (middle), EMC (right) and PHOS (left). 

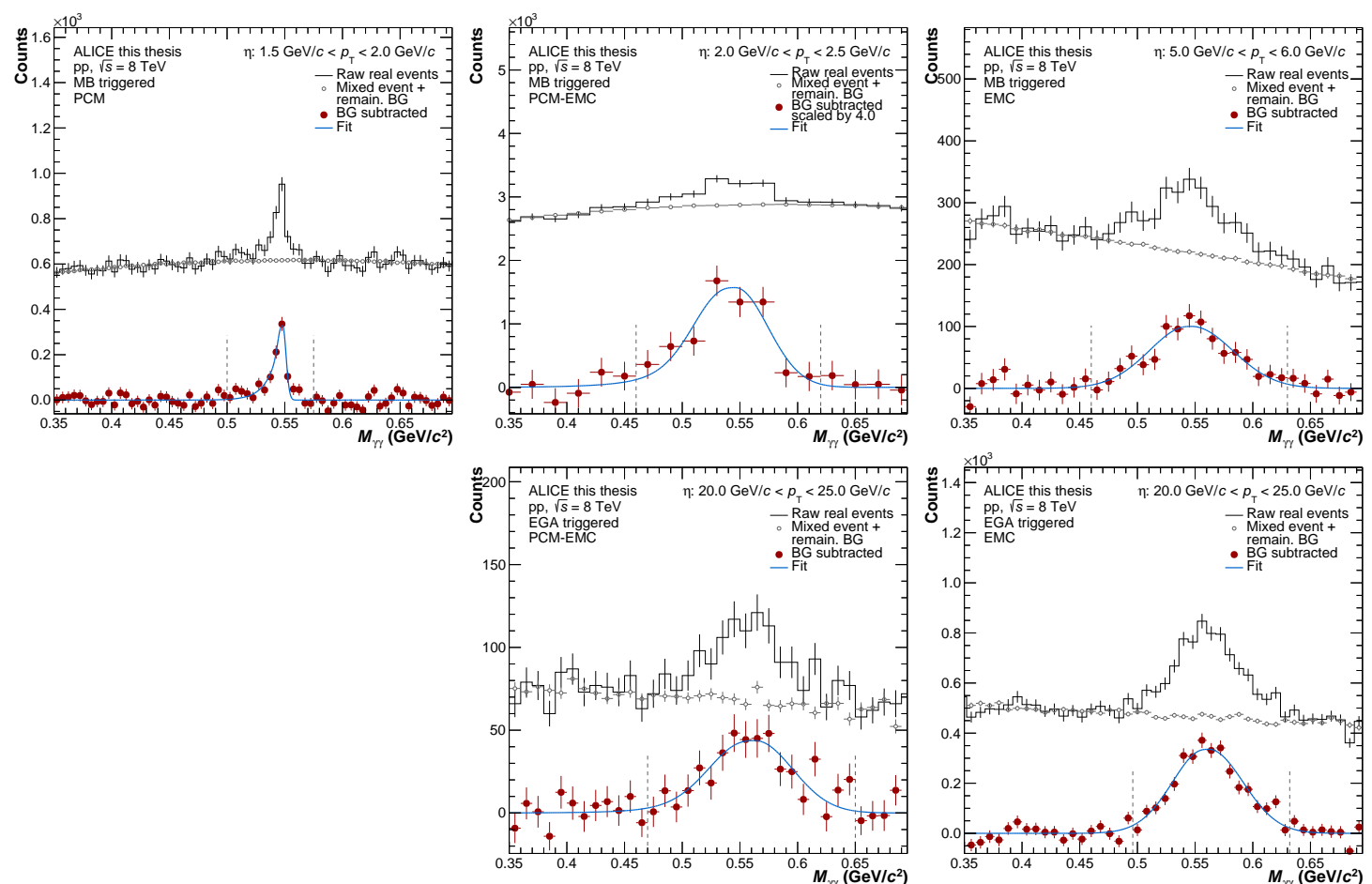

Figure A.2: Invariant mass distributions in a wide window around the nominal $\eta$ meson mass for pp collisions at $\sqrt{s}=8 \mathrm{TeV}$ minimum bias data (top) and triggered data (bottom) for PCM (left), PCM-EMC (middle) and EMC (right).

\section{A.3 Invariant mass peak position and width in pp collisions}
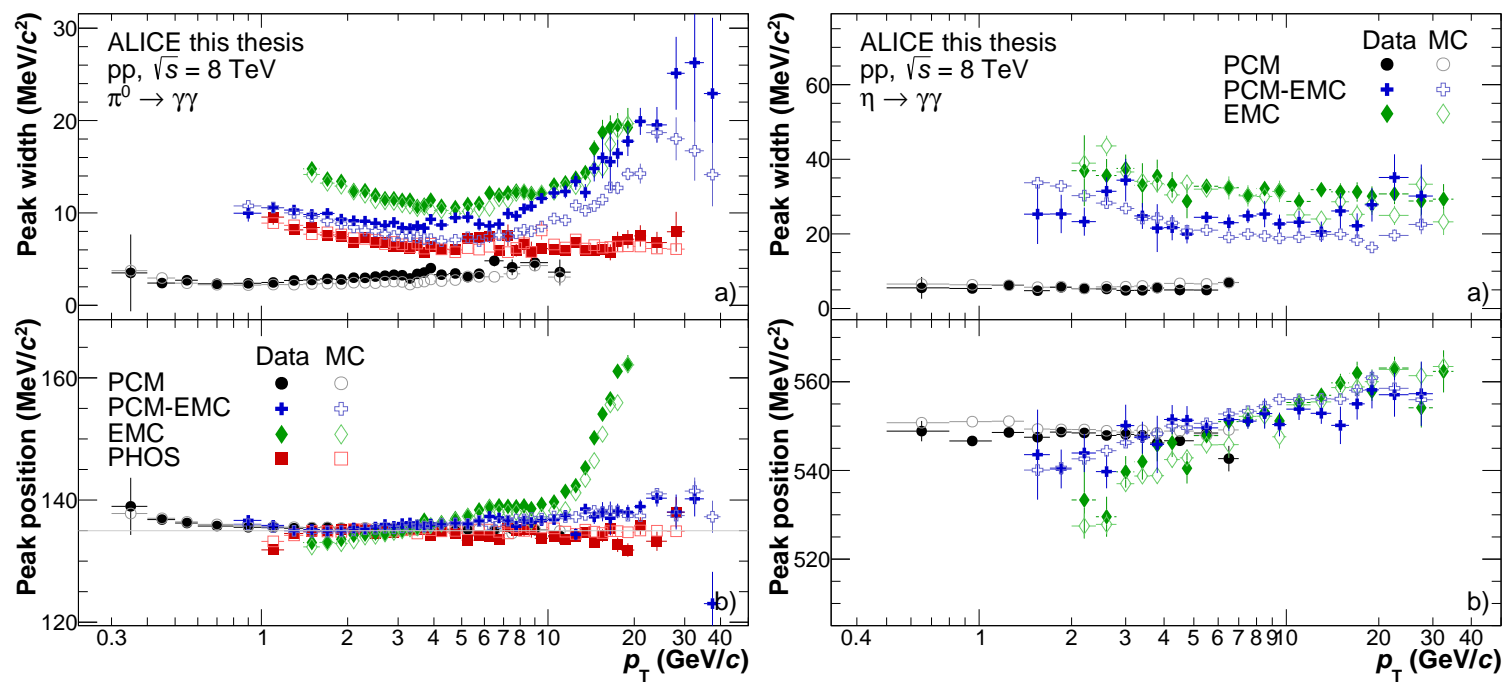

Figure A.3: Invariant mass peak positions (bottom panels) and width (top panels) obtained from the fits according to Equation 26 for the $\pi^{0}$ (left) and $\eta$ meson (right) versus transverse momentum for pp collisions at $\sqrt{s}=8 \mathrm{TeV}$. Data is shown in full markers for PCM (black), PCM-EMC (blue), EMC (green) and PHOS (red) while the corresponding MC is shown in open markers. 


\section{A.4 Secondary neutral pion correction factors}
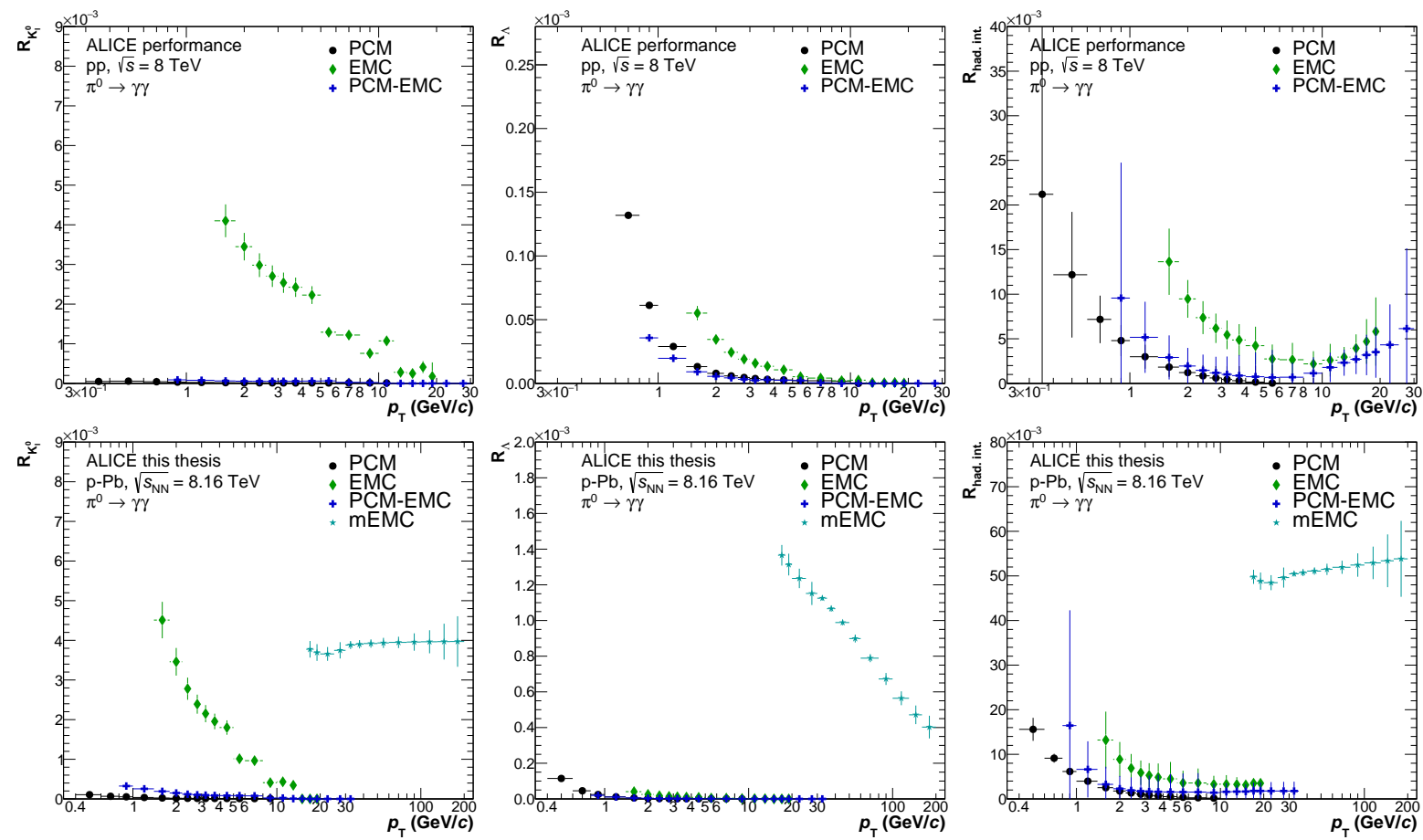

Figure A.4: Effective correction for secondary $\pi^{0}$ from $\mathrm{K}_{\mathrm{L}}^{0}$ (left) and $\Lambda$ (middle) decays as well as from hadronic interactions in the detector material (right). Corrections are shown for PCM, PCM-EMC, EMC and mEMC in pp collisions at $\sqrt{s}=8 \mathrm{TeV}($ top $)$ and in $\mathrm{p}-\mathrm{Pb}$ collisions at $\sqrt{s_{\mathrm{NN}}}=8.16 \mathrm{TeV}($ bottom $) ..$ 


\section{A.5 Correlations and weights for combination of triggers}
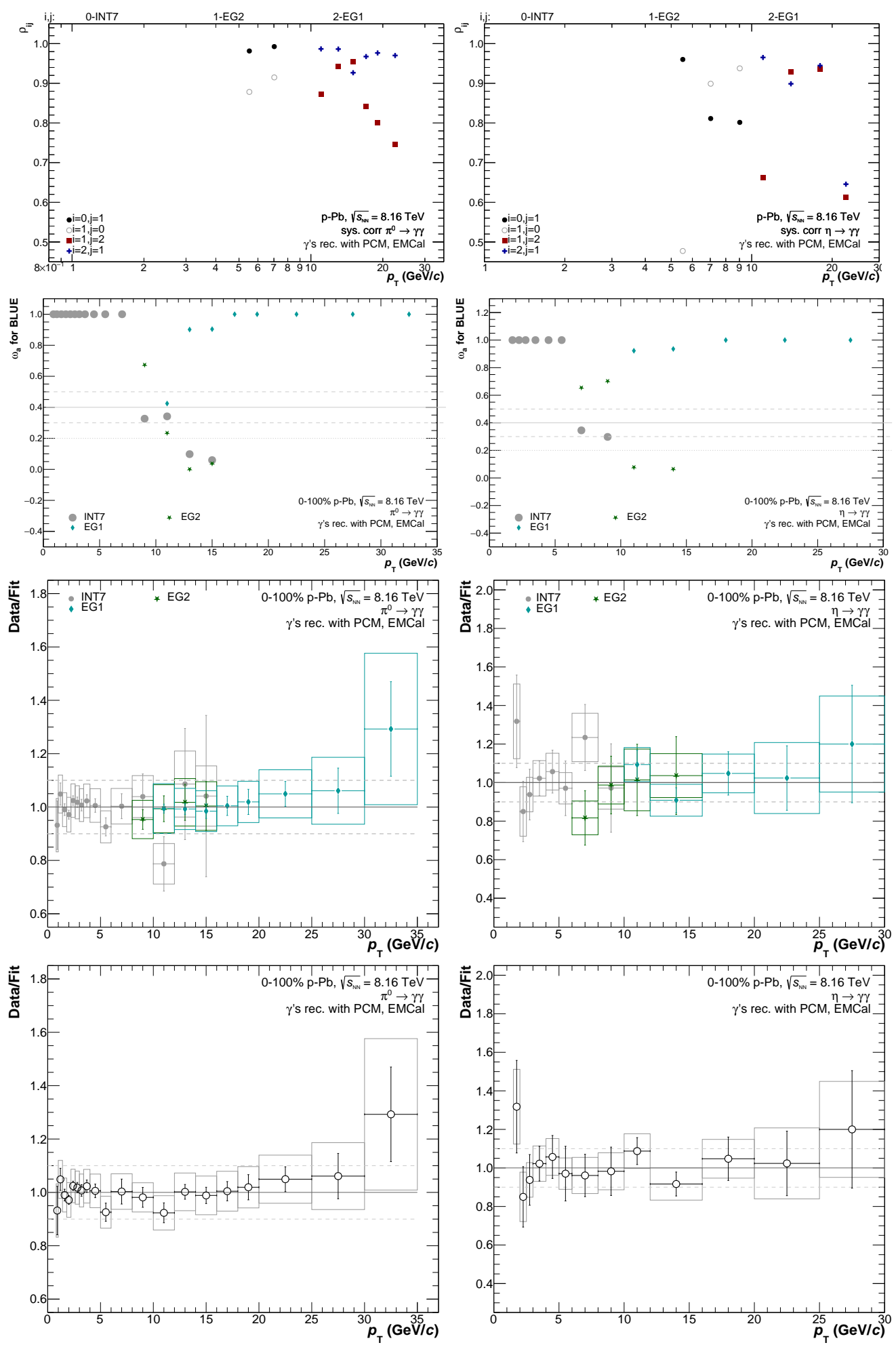

Figure A.5: From top to bottom: a) Correlation factors $\rho_{i j}$ between the different triggers used in the PCM-EMC analysis in $\mathrm{p}-\mathrm{Pb}$ collisions at $\sqrt{s_{\mathrm{NN}}}=8.16 \mathrm{TeV}$. b) Weights $\omega_{a}$ determined within the BLUE method for the $\pi^{0}$ (left) and the $\eta$ (right) spectrum. $c$ ) Ratio of the $R F$-scaled $\pi^{0}$ spectra of each EMCal trigger to a combined TCM fit. d) Ratio of the combined spectra to the same TCM fit. 

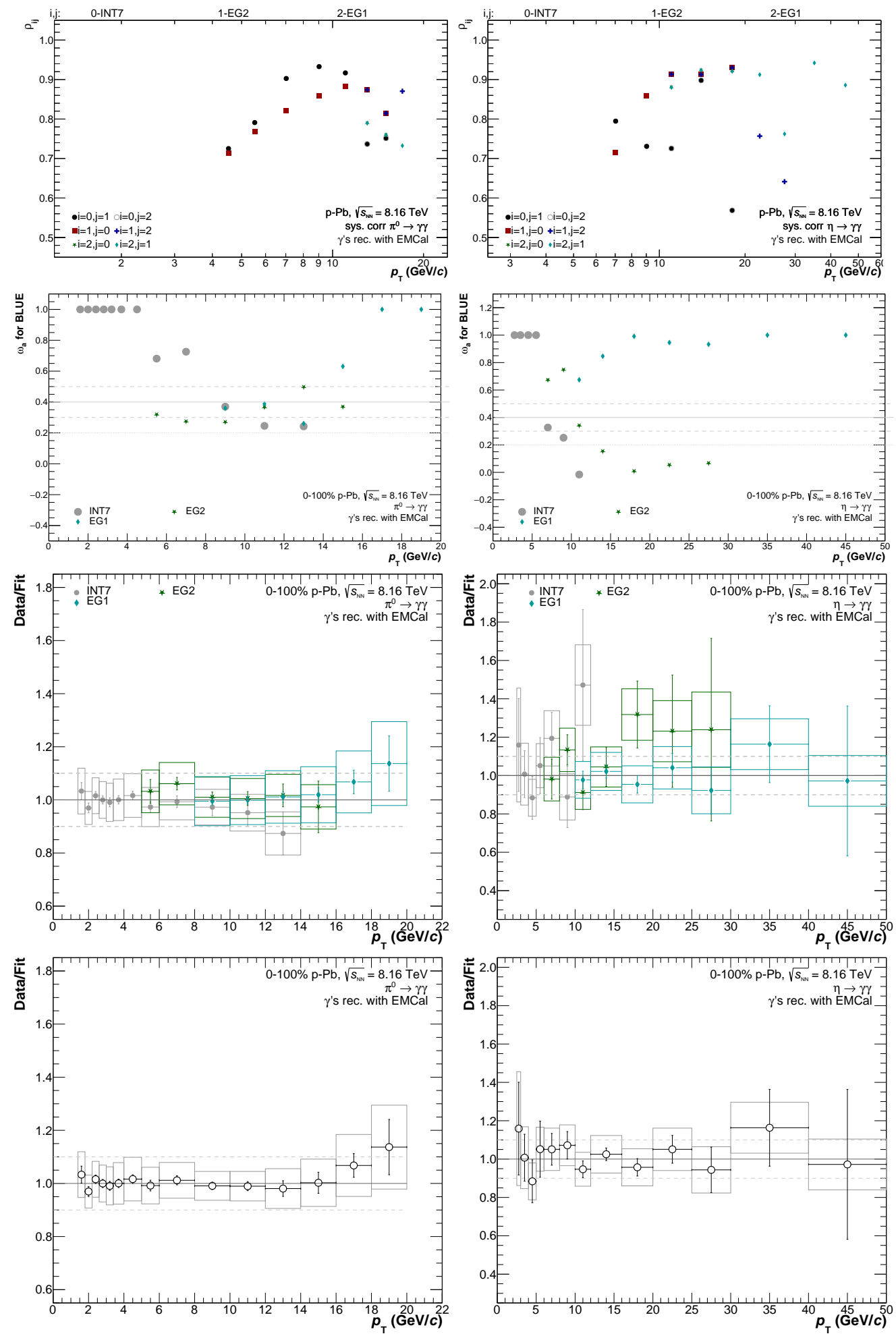

Figure A.6: From top to bottom: a) Correlation factors $\rho_{i j}$ between the different triggers used in the EMC analysis in $\mathrm{p}-\mathrm{Pb}$ collisions at $\sqrt{s_{\mathrm{NN}}}=8.16 \mathrm{TeV}$. b) Weights $\omega_{a}$ determined within the BLUE method for the $\pi^{0}$ (left) and the $\eta$ (right) spectrum. c) Ratio of the $R F$-scaled $\pi^{0}$ spectra of each EMCal trigger to a combined TCM fit. d) Ratio of the combined spectra to the same TCM fit. 


\section{A.6 Systematic uncertainties}

\begin{tabular}{|c|c|c|c|c|c|c|c|c|c|c|c|c|c|}
\hline$p_{\mathrm{T}}$ interval & & $4-1$ & $8 \mathrm{Ge}$ & & & $0-6$ & $0 \mathrm{Ge}$ & & & $0-1$ & $3.0 \mathrm{G}$ & & $100-130.0 \mathrm{GeV} / c$ \\
\hline Method & $\mathrm{PCM}$ & $\begin{array}{l}\mathrm{PCM} \\
\mathrm{EMC}\end{array}$ & EMC & PHOS & $\mathrm{PCM}$ & $\begin{array}{l}\mathrm{PCM} \\
\mathrm{EM}\end{array}$ & EMC & PHOS & $\begin{array}{l}\text { PCM } \\
\text { EMC }\end{array}$ & EMC & PHO & $11 \mathrm{~T}$ & $\mathrm{mEMC}$ \\
\hline$\overline{\overline{\text { Signal extraction }}}$ & 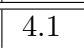 & 2.4 & 3.5 & 2.1 & $\overline{66.9}$ & 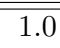 & 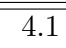 & 2.9 & 1.5 & 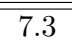 & 4.9 & 8.3 & 9.5 \\
\hline Inner material & 9.0 & 4.5 & - & - & 9.0 & 4.5 & - & - & 4.5 & - & - & - & - \\
\hline Outer material & - & 2.8 & 4.2 & 2.0 & - & 2.8 & 4.2 & 2.0 & 2.8 & 4.2 & 2.0 & 2.1 & 2.1 \\
\hline PCM track rec. & 0.2 & 0.9 & - & - & 0.4 & 0.8 & - & _- & 0.4 & - & - & - & - \\
\hline PCM electron PID & 0.7 & 0.4 & - & - & 0.6 & 0.5 & - & - & 0.7 & - & - & - & - \\
\hline PCM photon PID & 0.7 & 0.9 & - & - & 1.0 & 2.0 & - & - & 2.1 & - & - & - & - \\
\hline Cluster description & - & 1.5 & 5.5 & 1.1 & - & 2.0 & 3.6 & 0.1 & 2.9 & 5.2 & - & 3.8 & 4.3 \\
\hline Cluster energy calib. & - & 1.5 & 1.9 & 2.3 & - & 1.5 & 1.9 & 3.1 & 1.5 & 1.9 & 3.2 & 3.3 & 3.3 \\
\hline Track match to cluster & - & 0.2 & 0.2 & - & - & 0.7 & 0.3 & - & 0.5 & 1.1 & - & - & - \\
\hline Efficiency & - & 1.0 & 2.3 & 1.9 & - & 1.0 & 2.3 & 1.9 & 1.0 & 3.0 & 2.0 & 3.6 & 3.6 \\
\hline Trigg. norm.\&pileup & 4.7 & - & - & 2.0 & 3.3 & - & - & 2.0 & 2.8 & 2.8 & 2.8 & 1.9 & 1.9 \\
\hline Total syst. uncertainty & 11.0 & 6.4 & 8.3 & 5.5 & 11.9 & 6.5 & 7.6 & 5.2 & 7.4 & 10.9 & 7.0 & 10.7 & 11.9 \\
\hline Statistical uncertainty & 2.2 & 2.2 & 3.1 & 1.1 & 6.7 & 3.7 & 2.0 & 3.1 & 3.6 & 4.2 & 3.6 & 2.4 & 5.7 \\
\hline Combined stat. unc. & & & 0 & & & & .8 & & & & .2 & & 5.7 \\
\hline Combined syst. unc. & & & 6 & & & & .9 & & & & .9 & & 11.9 \\
\hline
\end{tabular}

Table 15: Summary of relative systematic uncertainties in percent for selected $p_{\mathrm{T}}$ bins for the reconstruction of $\pi^{0}$ mesons in p-Pb collisions at $\sqrt{s}=8 \mathrm{TeV}$. The statistical uncertainties are given in addition to the total systematic uncertainties for each bin. Moreover, the combined statistical and systematic uncertainties are also listed, obtained by applying the BLUE method $[209,210]$ for all reconstruction methods available in the given $p_{\mathrm{T}}$ bin, considering the uncertainty correlations for the different methods. The uncertainty from $\sigma_{\mathrm{MB}}$ determination of $1.9 \%$, see Ref. [179], is independent of the reported measurements and is separately indicated in the figures.

\begin{tabular}{|c|c|c|c|c|c|c|c|c|c|c|c|}
\hline$p_{\mathrm{T}}$ interval & & $.5-3$. & $0 \mathrm{GeV}$ & & & $.0-8$ & $0 \mathrm{GeI}$ & & $20.0-$ & -25.0 & $\mathrm{GeV} / c$ \\
\hline Method & PCM & $\begin{array}{l}\text { PCM- } \\
\text { EMC }\end{array}$ & EMC & PHOS & $\mathrm{PCM}$ & $\begin{array}{l}\mathrm{PCM} \\
\mathrm{EMC}\end{array}$ & EMC & PHOS & $\begin{array}{l}\text { PCM- } \\
\text { EMC }\end{array}$ & $\mathrm{EM}$ & PHOS \\
\hline Signal extraction & 3.8 & 3.3 & 23.7 & 18.0 & 5.0 & 4.7 & 7.3 & 4.5 & 14.4 & 3.0 & 7.0 \\
\hline Inner material & 9.0 & 4.5 & - & - & 9.0 & 4.5 & - & - & 4.5 & - & - \\
\hline Outer material & - & 2.8 & 4.2 & 1.8 & - & 2.8 & 4.2 & 1.8 & 2.8 & 4.2 & 1.8 \\
\hline PCM track rec. & 1.2 & 1.9 & - & - & 2.0 & 2.8 & - & - & 1.3 & - & - \\
\hline PCM electron PID & 1.3 & 4.2 & - & - & 3.2 & 1.9 & - & - & 2.9 & - & - \\
\hline PCM photon PID & 2.6 & 3.9 & - & - & 4.1 & 4.7 & - & - & 6.0 & - & - \\
\hline Cluster description & - & 3.3 & 5.1 & 2.0 & - & 3.6 & 5.9 & 2.0 & 5.2 & 7.5 & 2.0 \\
\hline Cluster energy calib. & - & 1.5 & 6.7 & 3.1 & - & 1.5 & 4.2 & 3.2 & 1.5 & 3.3 & 3.2 \\
\hline Track match to cluster & - & 1.4 & 0.8 & - & - & 1.6 & 0.9 & - & 2.3 & 2.3 & - \\
\hline Efficiency & - & 1.0 & 2.3 & 1.6 & - & 1.0 & 2.5 & 1.6 & 1.0 & 2.6 & 1.6 \\
\hline Trigg. norm.\&pileup & 4.9 & - & - & - & 4.1 & 1.7 & 1.7 & 1.9 & 2.8 & 2.8 & 1.9 \\
\hline Total syst. uncertainty & 11.4 & 9.6 & 25.6 & 19.1 & 12.4 & 10.3 & 11.6 & 6.8 & 18.0 & 10.7 & 8.5 \\
\hline Statistical uncertainty & 12.0 & 14.2 & 20.8 & 29.7 & 25.9 & 11.4 & 7.9 & 6.5 & 16.4 & 6.9 & 15.9 \\
\hline Combined stat. unc. & & & .6 & & & & 5 & & & 8.2 & \\
\hline Combined syst. unc. & & & .6 & & & & 3 & & & 6.1 & \\
\hline
\end{tabular}

Table 16: Summary of relative systematic uncertainties in percent for selected $p_{\mathrm{T}}$ bins for the reconstruction of $\eta$ mesons, see Tab. 15 for further explanations which also apply here. 


\begin{tabular}{|c|c|c|c|c|c|c|c|c|c|c|c|}
\hline \multirow{2}{*}{\begin{tabular}{l|l}
$p_{\mathrm{T}}$ interval \\
Method \\
\end{tabular}} & \multicolumn{4}{|c|}{$2.5-3.0 \mathrm{GeV} / c$} & \multicolumn{4}{|c|}{$6.0-8.0 \mathrm{GeV} / c$} & \multicolumn{3}{|c|}{$20.0-25.0 \mathrm{GeV} / c$} \\
\hline & $\mathrm{PCM}$ & $\begin{array}{l}\text { PCM- } \\
\text { EMC }\end{array}$ & EMC & PHOS & $\mathrm{PCM}$ & $\begin{array}{l}\mathrm{PCM}- \\
\mathrm{EMC}\end{array}$ & EMC & $\mathrm{HOS}$ & $\begin{array}{l}\text { PCM- } \\
\text { EMC }\end{array}$ & & PHOS \\
\hline$\overline{\text { Signal extraction }}$ & 3.9 & 11.5 & 23.9 & 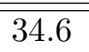 & 5.2 & 6.3 & $\overline{7.9}$ & 7.9 & 14.4 & 8.6 & 12.4 \\
\hline $\mathrm{PCM}$ & & 2.4 & - & - & 2.0 & 1.2 & - & 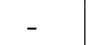 & 1.6 & 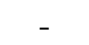 & - \\
\hline PCM & 6 & 5.1 & - & - & 1.3 & 1.6 & - & - & 3.0 & - & - \\
\hline $\mathrm{PCM} \mathrm{p}$ & 2.4 & 3.9 & - & - & 4.0 & 4.0 & - & - & 7.0 & - & - \\
\hline Cluster descr & - & 2.9 & 6.6 & - & - & 3.1 & 6.2 & - & 3.9 & 7.8 & - \\
\hline Clu & - & 1.5 & 3.0 & - & - & 1.5 & 3. & - & 1.5 & 3.0 & - \\
\hline & 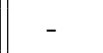 & 1.4 & 0. & - & - & 1.4 & 0. & - & 2.7 & 2.1 & - \\
\hline & 2.5 & 1.4 & 2.0 & 1.0 & 1.8 & 1.4 & 2.5 & 1.0 & 1.4 & 2.5 & 1.0 \\
\hline Total syst. uncertainty & 5.6 & 13.9 & 25.1 & 34.7 & 7.2 & 8.7 & 10.8 & 8.0 & 17.2 & 12.4 & 12.5 \\
\hline 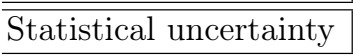 & 12.9 & 14.1 & 20.9 & 17.5 & 25.9 & 14.0 & 8.1 & 5.8 & 16.9 & 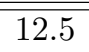 & $\overline{12.9}$ \\
\hline 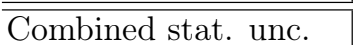 & & & & & & & .5 & & & 8.2 & \\
\hline Combined syst. unc. & & & 6 & & & & 3 & & & 6.1 & \\
\hline
\end{tabular}

Table 17: Summary of relative systematic uncertainties in percent for selected $p_{\mathrm{T}}$ bins for the determination of the $\eta / \pi^{0}$ ratio. The statistical uncertainties are given in addition to the total systematic uncertainties for each bin. Moreover, the combined statistical and systematic uncertainties are listed as well, see also explanations in caption of Tab. 15.

\begin{tabular}{|c|c|c|c|c|c|c|c|c|c|c|c|c|c|}
\hline$p_{\mathrm{T}}$ interval & & $1.4-1$ & $8 \mathrm{GeV}$ & & & $.0-6$ & $\mathrm{GeV}$ & & & $.0-1$ & $8.0 \mathrm{Ge}$ & & $100-130.0 \mathrm{GeV} / c$ \\
\hline Method & $\mathrm{PCM}$ & $\begin{array}{l}\text { PCM } \\
\text { EMO }\end{array}$ & EMC & PHOS & $\mathrm{PCM}$ & $\begin{array}{l}\text { PCM- } \\
\text { EMC }\end{array}$ & EMC & PHOS & $\begin{array}{l}\text { PCM- } \\
\text { EMC }\end{array}$ & EN & PHOS & nEMC & mEMC \\
\hline Signal extraction & 2.6 & 2.9 & 5.1 & 7.6 & 6.9 & 1.6 & 4.7 & 7.6 & 2.2 & 7.0 & 7.6 & 1.0 & 1.0 \\
\hline PCM track rec. & 0.2 & 0.2 & - & 7.6 & 0.5 & 0.2 & - & 7.6 & 1.5 & - & 7.6 & - & - \\
\hline PCM electron PID & 0.3 & 0.9 & - & 7.6 & 0.5 & 0.9 & - & 7.6 & 1.0 & - & 7.6 & - & - \\
\hline PCM photon PID & 0.7 & 0.9 & - & 7.6 & 1.4 & 1.9 & - & 7.6 & 2.7 & - & 7.6 & - & - \\
\hline Cluster description & - & 1.0 & 4.7 & 7.6 & - & 1.5 & 2.5 & 7.6 & 1.8 & 4.1 & 7.6 & 2.0 & 3.1 \\
\hline Cluster energy calib. & - & 1.5 & 0.8 & 7.6 & - & 1.5 & 0.8 & 7.6 & 1.5 & 0.8 & 7.6 & 1.0 & 1.0 \\
\hline Track match to cluster & - & 0.2 & 0.2 & 7.6 & - & 0.4 & 0.3 & 7.6 & 2.9 & 1.1 & 7.6 & 0.5 & 1.5 \\
\hline Efficiency & - & 0.5 & 1.7 & 7.6 & - & 0.5 & 1.7 & 7.6 & 0.5 & 1.7 & 7.6 & 0.9 & 0.9 \\
\hline Trigg. norm.\&pileup & 5.2 & - & - & 7.6 & 6.3 & 3.2 & - & 7.6 & 4.9 & 4.2 & 7.6 & 3.7 & 3.7 \\
\hline Total syst. uncertainty & 6.5 & 3.7 & 7.2 & 7.6 & 9.5 & 5.7 & 5.6 & 7.6 & 7.3 & 9.3 & 7.6 & 4.6 & 5.4 \\
\hline Statistical uncertainty & 3.0 & 2.6 & 4.4 & 6.9 & 9.2 & 5.7 & 3.1 & 2.7 & 5.1 & 5.3 & 11.7 & 2.7 & 10.8 \\
\hline Combined stat. unc. & & & .8 & & & & .7 & & & & 2.2 & & 10.8 \\
\hline Combined syst. unc. & & & .7 & & & & .9 & & & & 2.9 & & 5.4 \\
\hline
\end{tabular}

Table 18: Summary of relative systematic uncertainties in percent for selected $p_{\mathrm{T}}$ bins for the determination of the $\pi^{0}$ meson nuclear modification factor $R_{\mathrm{pA}}$. The statistical uncertainties are given in addition to the total systematic uncertainties for each bin. Moreover, the combined statistical and systematic uncertainties are listed as well, see also explanations in caption of Tab. 15 . 


\begin{tabular}{|c|c|c|c|c|c|c|c|c|}
\hline \multirow{2}{*}{\begin{tabular}{l|}
$p_{\mathrm{T}}$ interval \\
Method \\
\end{tabular}} & \multicolumn{3}{|c|}{$2.5-3.0 \mathrm{GeV} / c$} & \multicolumn{3}{|c|}{$6.0-8.0 \mathrm{GeV} / c$} & \multicolumn{2}{|c|}{$20.0-25.0 \mathrm{GeV} / \mathrm{c}$} \\
\hline & $\mathrm{PCM}$ & $\begin{array}{l}\text { PCM- } \\
\text { EMC }\end{array}$ & EMC & $\mathrm{PCM}$ & $\begin{array}{l}\text { PCM- } \\
\text { EMC }\end{array}$ & $\mathrm{EMC}$ & $\begin{array}{l}\text { PCM- } \\
\text { EMC }\end{array}$ & EMC \\
\hline Signal extraction & 4.2 & $\overline{5.5}$ & 29.4 & 6.3 & $\overline{5.4}$ & $\overline{8.3}$ & 8.3 & $\overline{4.8}$ \\
\hline PCM track rec. & 0.5 & 0.7 & 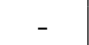 & 0.5 & 0.7 & 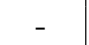 & 0.7 & - \\
\hline PCM electron PID & 0.4 & 1.2 & - & 0.7 & 1.9 & - & 3.4 & - \\
\hline PCM photon PID & 1.9 & 4.8 & - & 2.9 & 5.1 & - & 5.1 & - \\
\hline Cluster description & 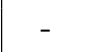 & 2.4 & 6.6 & - & 2.8 & 6.2 & 9.1 & 7.6 \\
\hline Cluster energy calib. & - & 1.5 & 2.0 & - & 1.5 & 2.0 & 1.5 & 2.0 \\
\hline Track match to cluster & - & 0.5 & 0.8 & - & 0.7 & 0.9 & 2.9 & 2.3 \\
\hline Efficiency & - & 0.2 & 2.6 & - & 0.2 & 2.6 & 0.2 & 2.9 \\
\hline Trigg. norm.\&pileup & 6.1 & - & - & 6.1 & 3.2 & 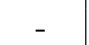 & 4.9 & 4.2 \\
\hline Total syst. uncertainty & 7.7 & 8.0 & 30.3 & 9.3 & 9.0 & 10.9 & 15.0 & 10.8 \\
\hline 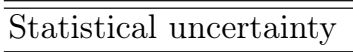 & 18.7 & 18.2 & 23.9 & 37.3 & 19.8 & \begin{tabular}{|c|}
15.4 \\
\end{tabular} & 21.9 & 27.8 \\
\hline 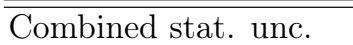 & & 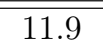 & & & 111.6 & & & $\overline{7.2}$ \\
\hline Combined syst. unc. & & 5.9 & & & 6.8 & & & 1.5 \\
\hline
\end{tabular}

Table 19: Summary of relative systematic uncertainties in percent for selected $p_{\mathrm{T}}$ bins for the determination of the $\eta$ meson nuclear modification factor $R_{\mathrm{pA}}$. The statistical uncertainties are given in addition to the total systematic uncertainties for each bin. Moreover, the combined statistical and systematic uncertainties are listed as well, see also explanations in caption of Tab. 15 .

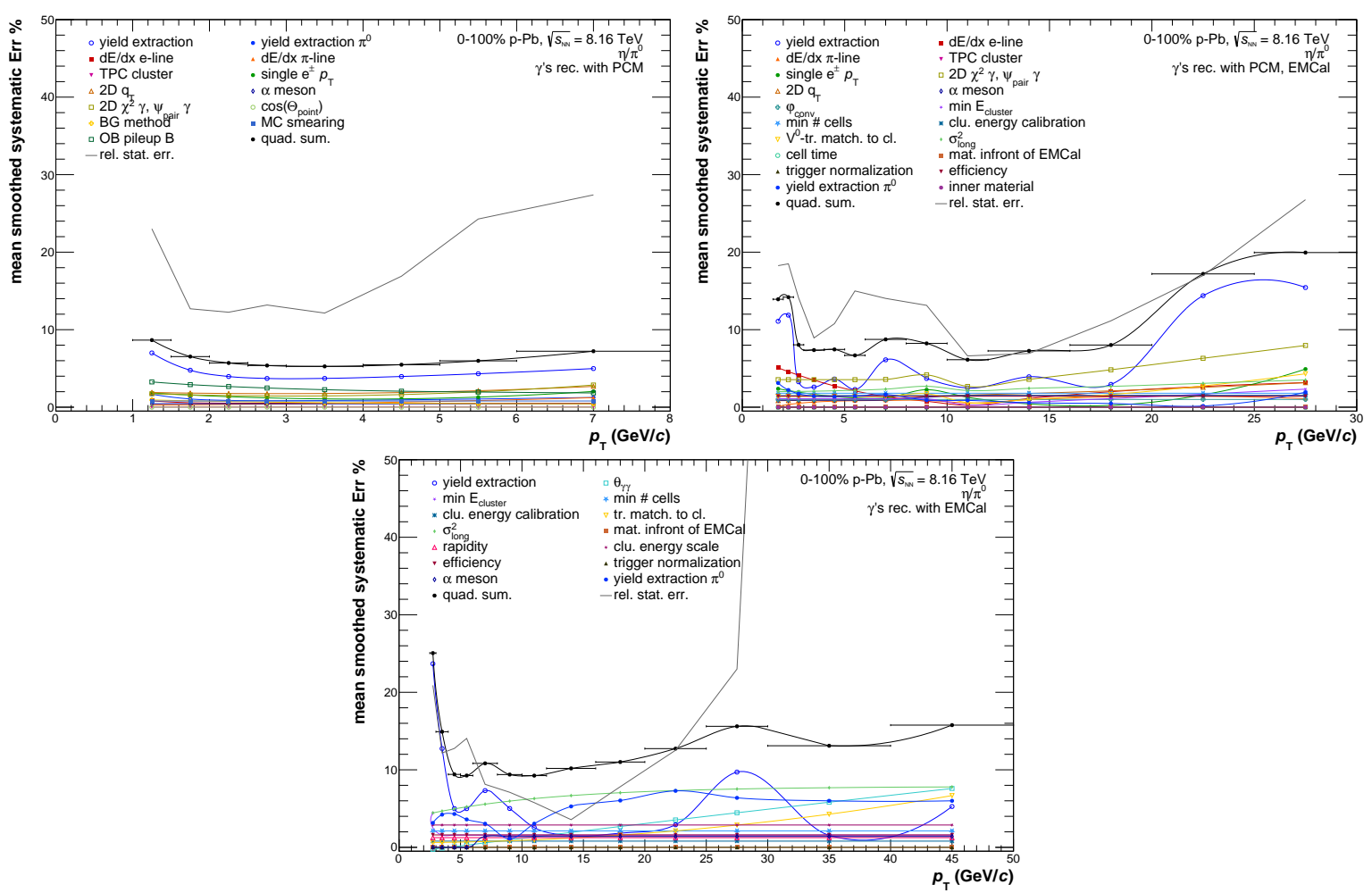

Figure A.8: Visualization of the systematic errors for the $\eta$ to $\pi^{0}$ ratio for PCM, PCM-EMC and EMC. The colored points represent the individual error sources, while the black points represent the final systematic error. The EMC plot is only valid up to $20 \mathrm{GeV} / c$, due to limitations in the framework, the plot was made up to $50 \mathrm{GeV} / c$. 

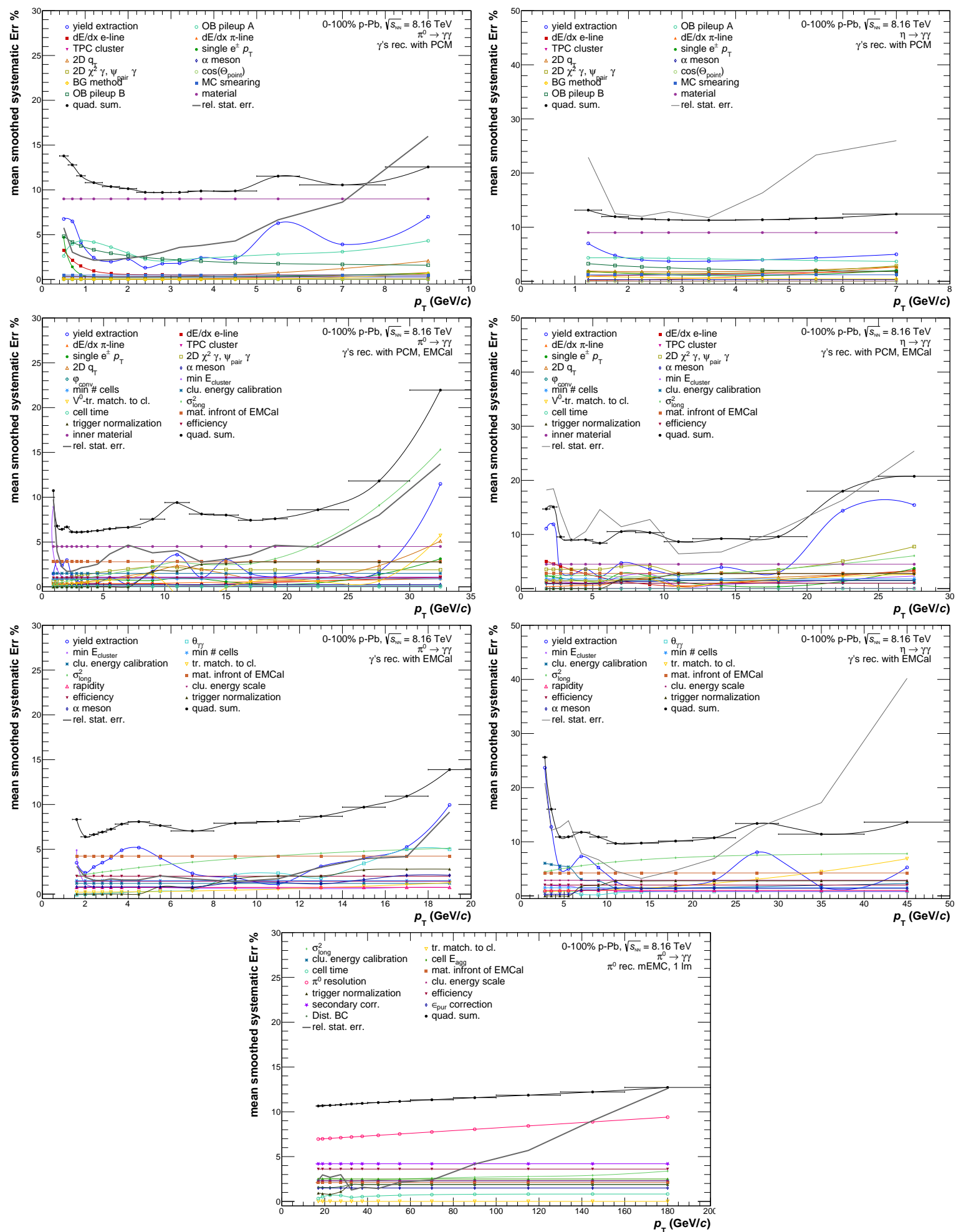

Figure A.7: Visualization of the systematic errors for the $\pi^{0}$ (left) and $\eta$ (right) meson spectra. The colored points represent the individual error sources, while the black points represent the final systematic error. 

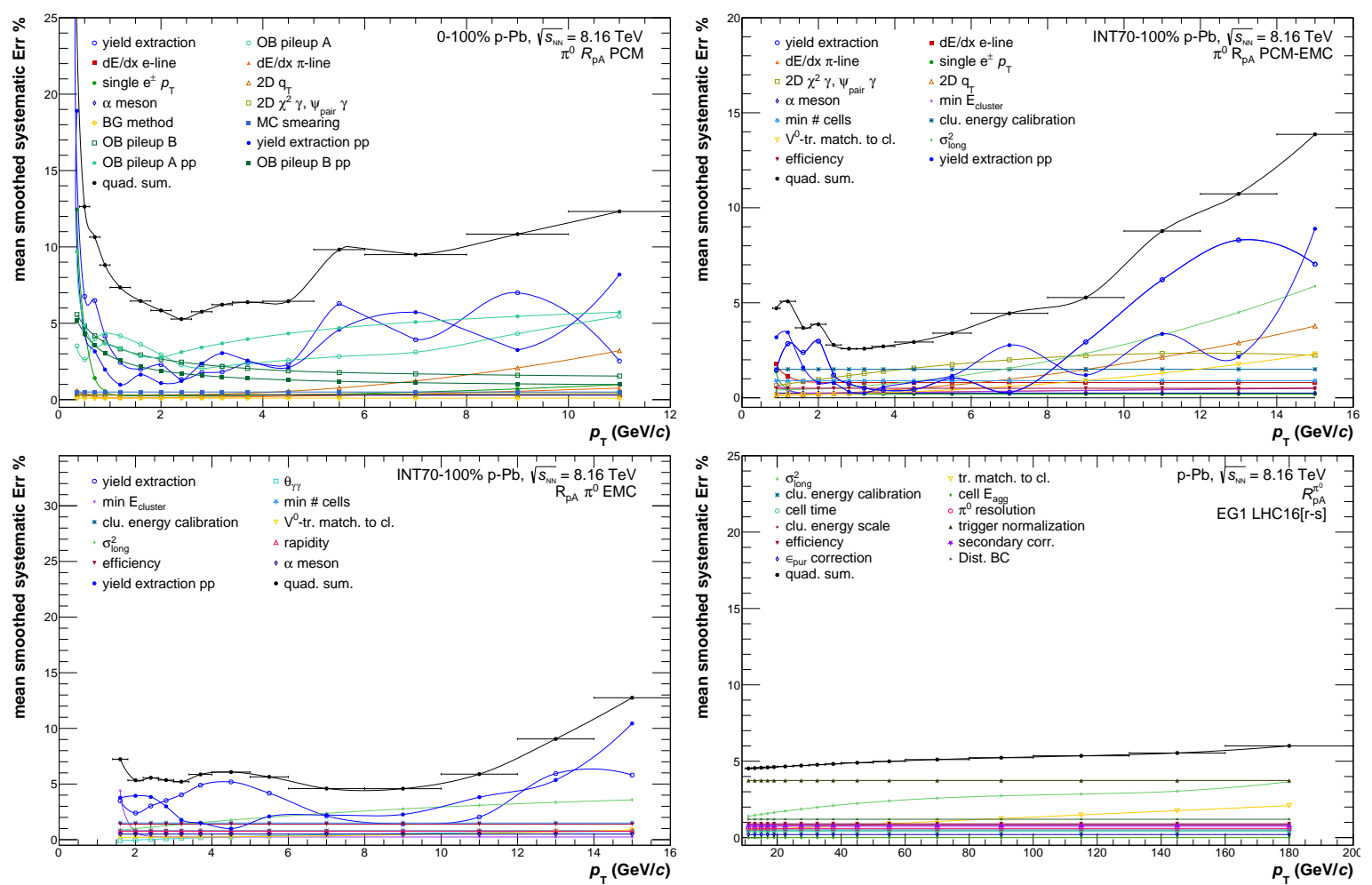

Figure A.9: Visualization of the systematic errors for the nuclear modification factor $R_{\mathrm{pA}}$ of the $\pi^{0}$ meson. The colored points represent the individual error sources, while the black points represent the final systematic error.

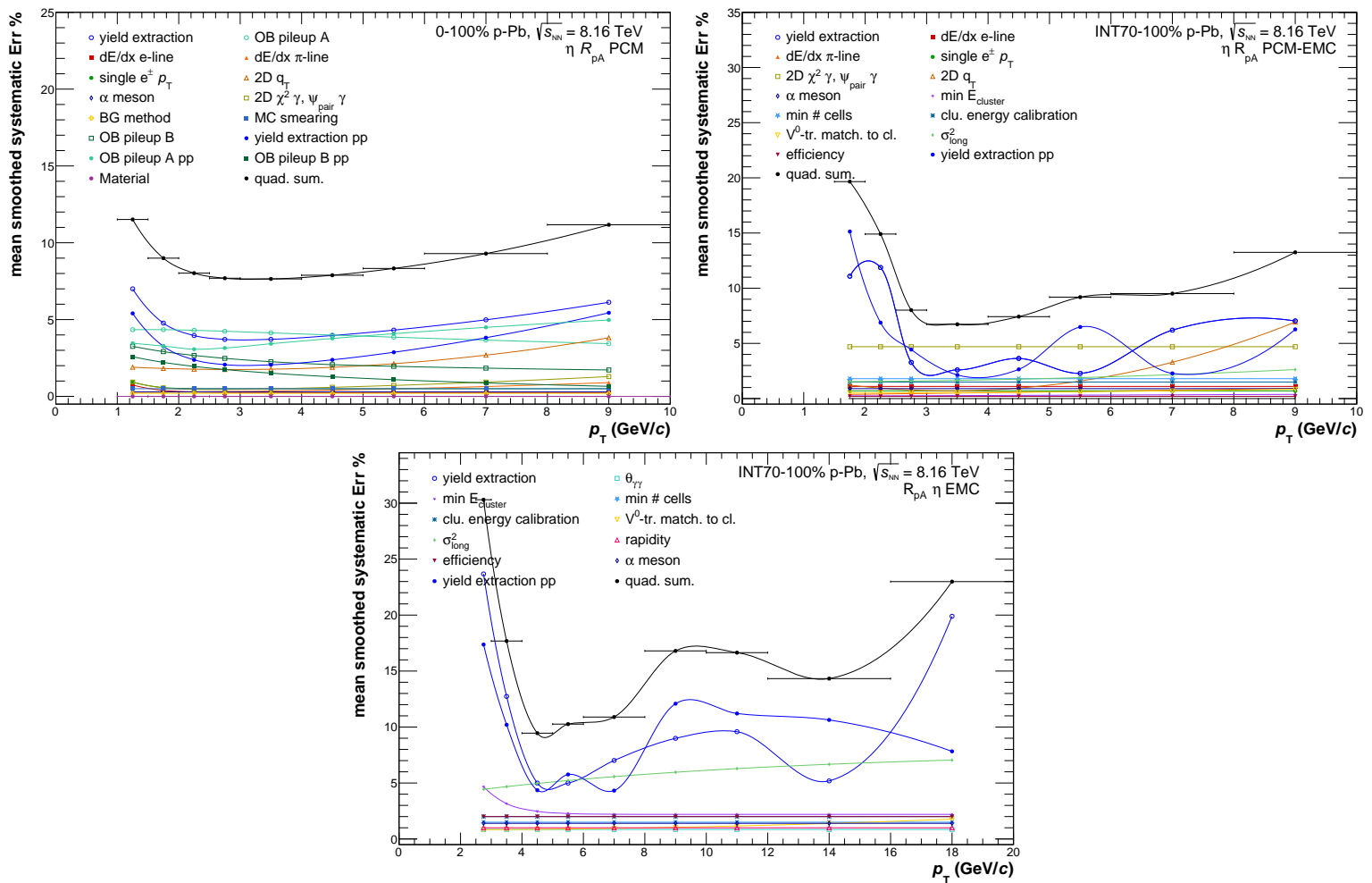

Figure A.10: Visualization of the systematic errors for the nuclear modification factor $R_{\mathrm{pA}}$ of the $\eta$ meson. The colored points represent the individual error sources, while the black points represent the final systematic error. 


\section{A.7 Combination of spectra}

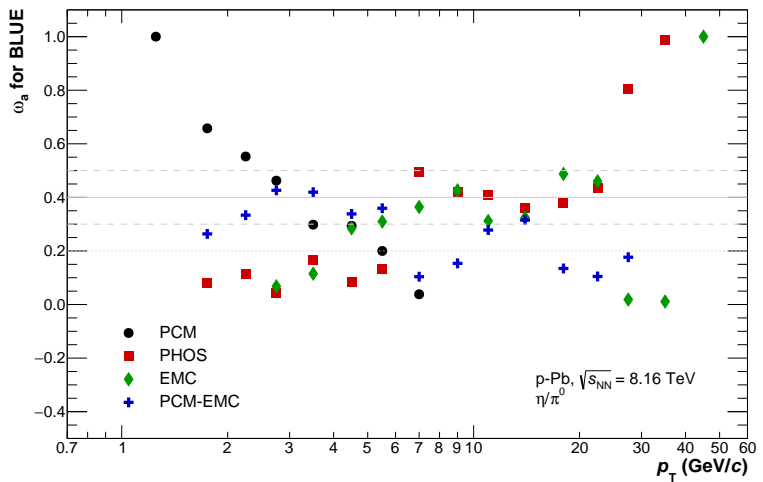

Figure A.11: Weights used in the BLUE method for the combination of the $\eta / \pi^{0}$ ratios from the different reconstruction techniques versus transverse momentum.
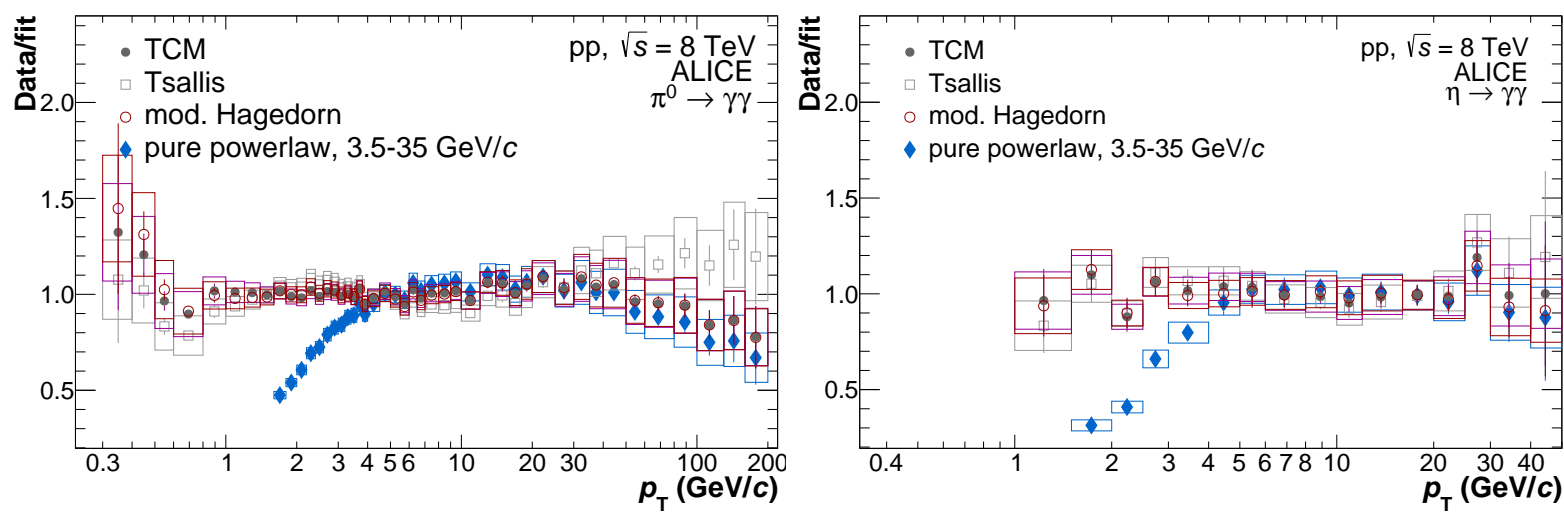

Figure A.12: Ratio of the combined $\pi^{0}$ (left) and $\eta$ meson (right) cross section spectra in pp to four different fit functions. The comparison to a two-component model fit [216, 216-218] is shown in gray circles while a Levy-Tsallis fit [215] comparison is shown in open gray squares. In addition, comparisons to a modified Hagedorn function [219] as well as a power-law for the high momentum region of $p_{\mathrm{T}}>3.5 \mathrm{GeV} / c$ is given.
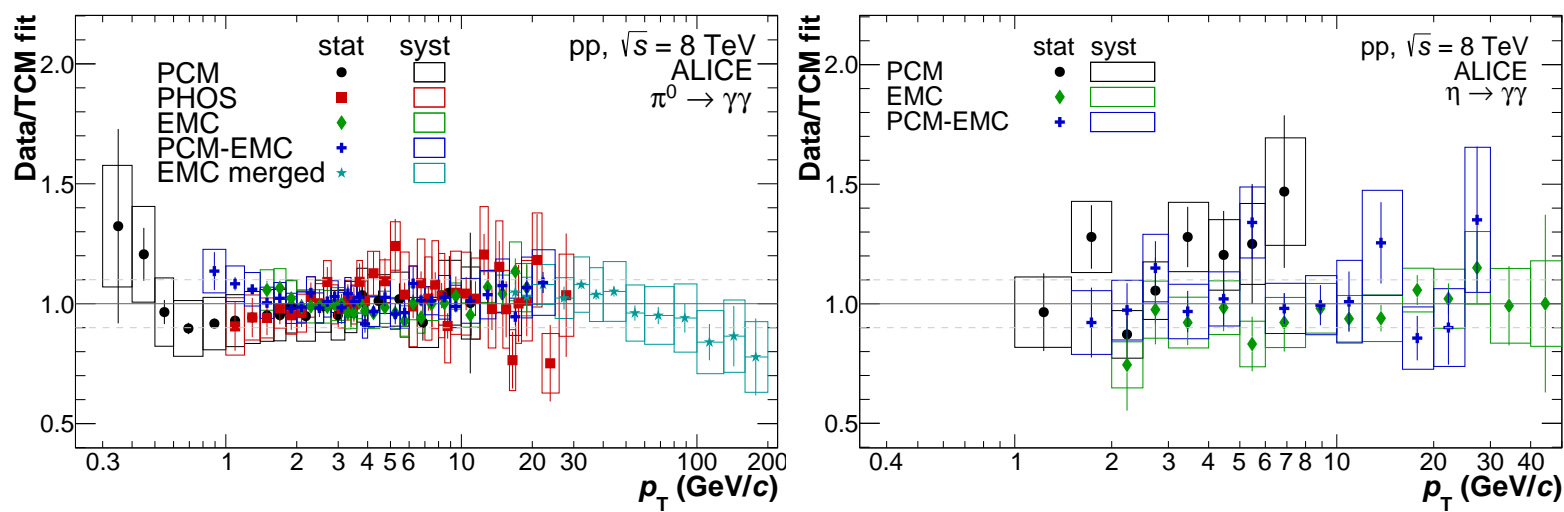

Figure A.13: Ratio of the individual $\pi^{0}$ (left) and $\eta$ meson (right) cross section spectra in pp to the two-component model fit of the respective combined spectra. 

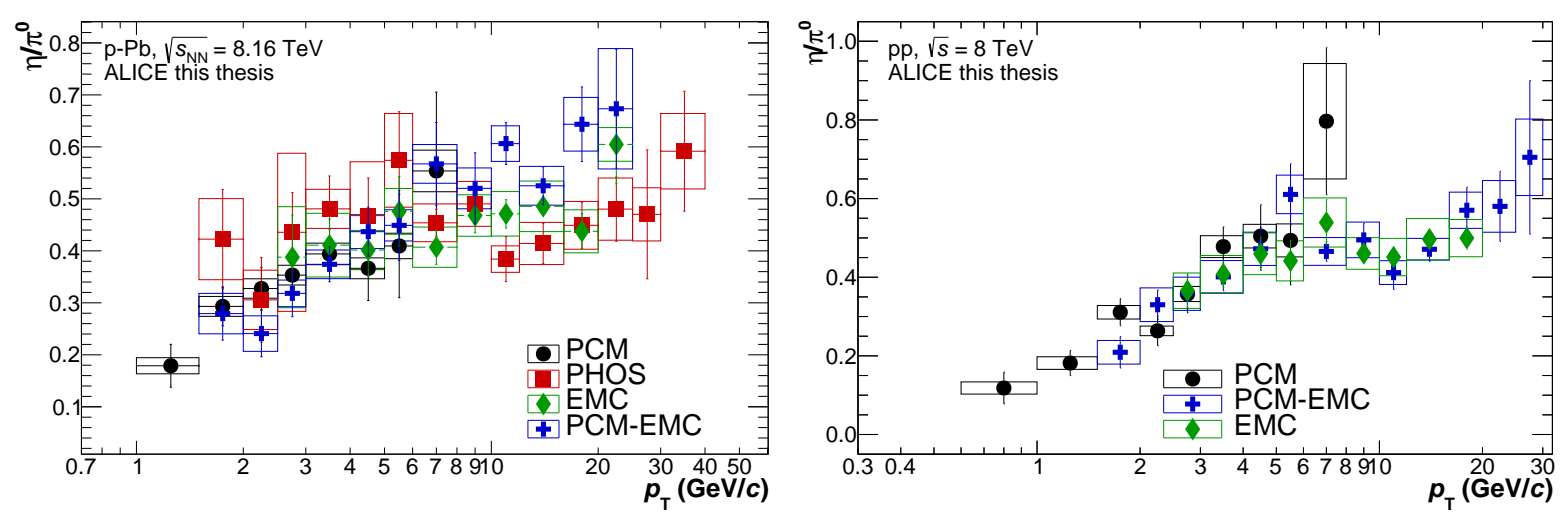

Figure A.14: Comparison of the individual $\eta / \pi^{0}$ ratios in $\mathrm{p}-\mathrm{Pb}$ (left) and pp (right).

\section{A.8 Nuclear modification factor}
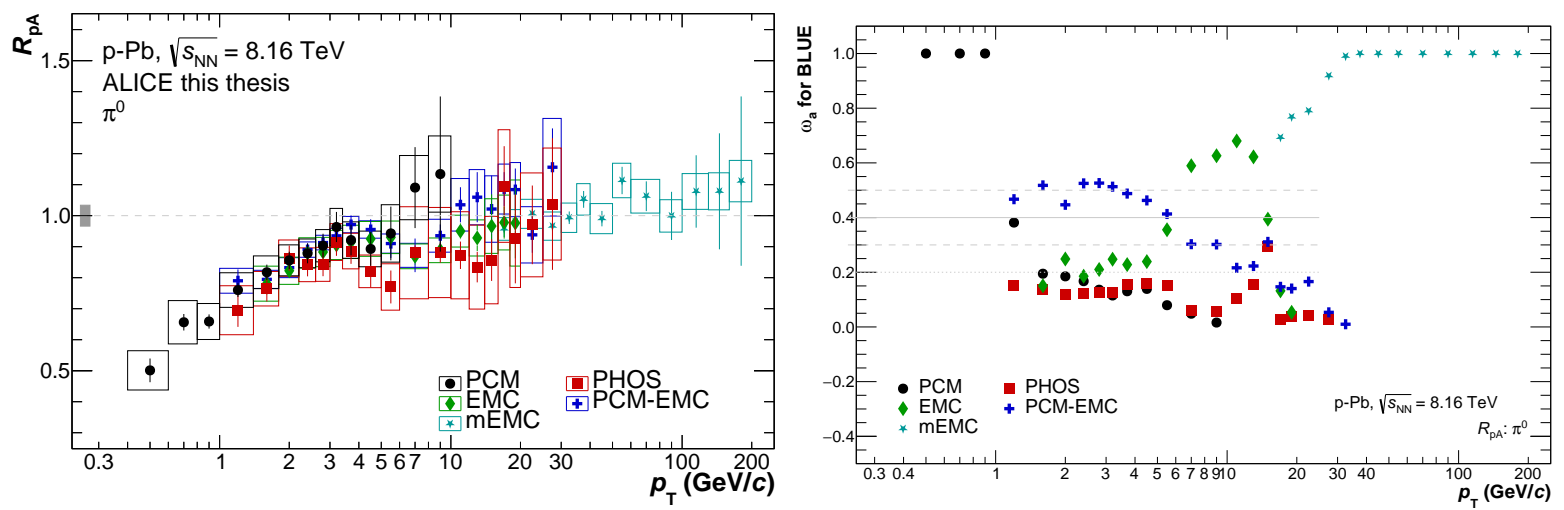

Figure A.15: Comparison of the individual $\pi^{0}$ meson nuclear modification factors in $\mathrm{p}-\mathrm{Pb}$ $\sqrt{s_{\mathrm{NN}}}=8.16 \mathrm{TeV}$ (left) and the weights used in the BLUE method combination procedure (right).
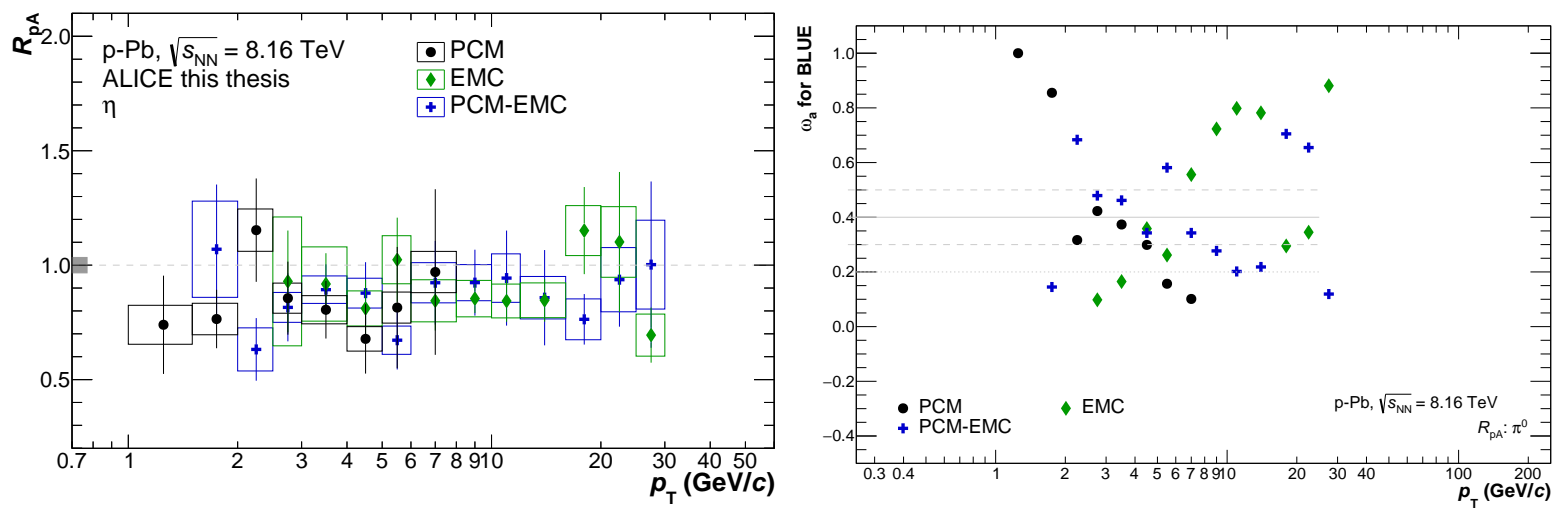

Figure A.16: Comparison of the individual $\eta$ meson nuclear modification factors in $\mathrm{p}-\mathrm{Pb}$ $\sqrt{s_{\mathrm{NN}}}=8.16 \mathrm{TeV}$ (left) and the weights used in the BLUE method combination procedure (right). 


\section{A.9 Direct and inclusive photons}

\section{A.9.1 Particle decay simulations}
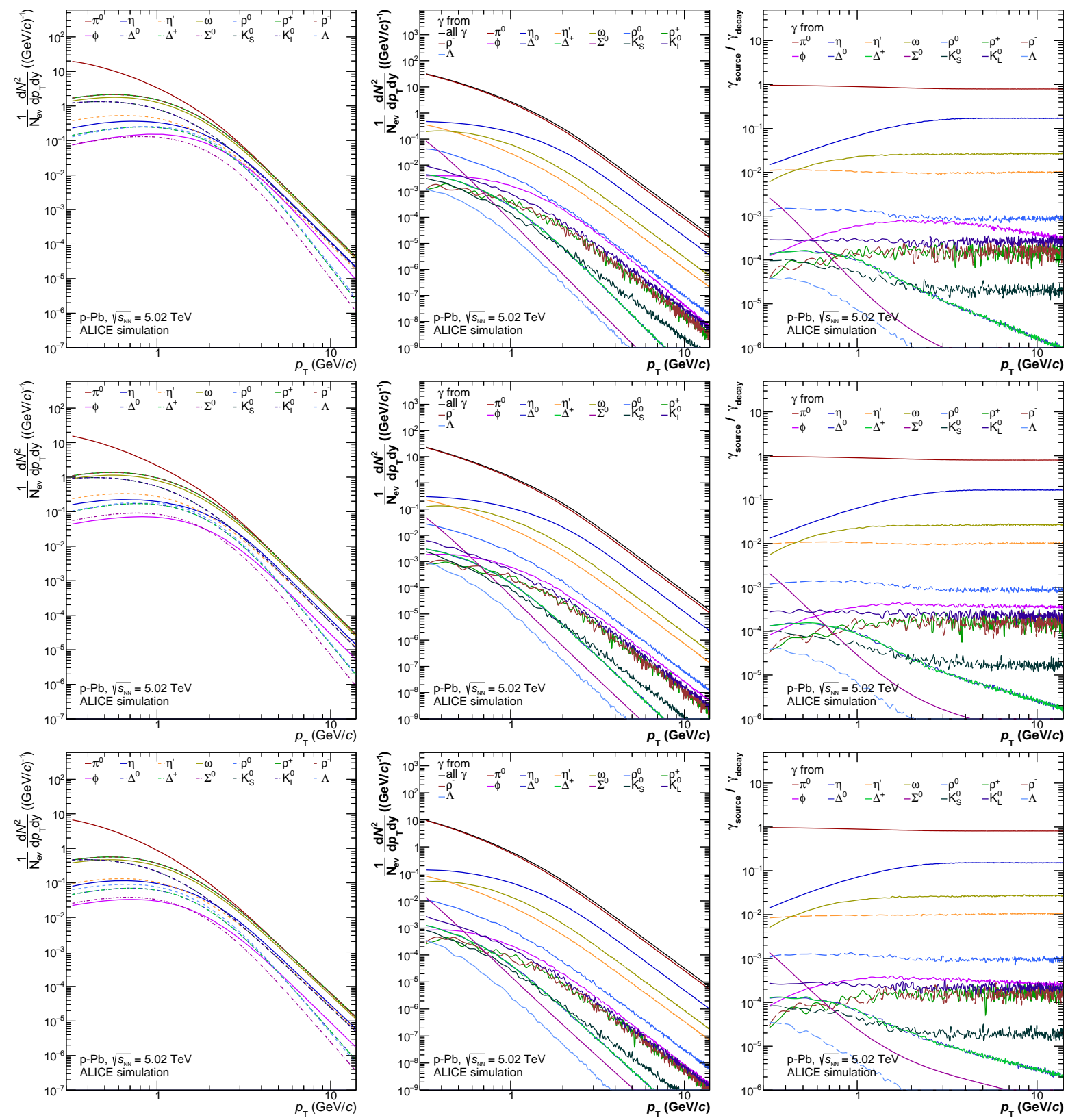

Figure A.17: Particle decay simulation mother particle spectra (left), decay photon spectra (middle) and ratio of decay photons from different sources to all decay photons (right) for $\mathrm{p}-\mathrm{Pb}$ collisions at $\sqrt{s_{\mathrm{NN}}}=5.02 \mathrm{TeV}$ at $20-40 \%$ multiplicity (top), 40-60\% multiplicity (midlle) and 60-100\% multiplicity (bottom). 


\section{A.9.2 Weights for combination}
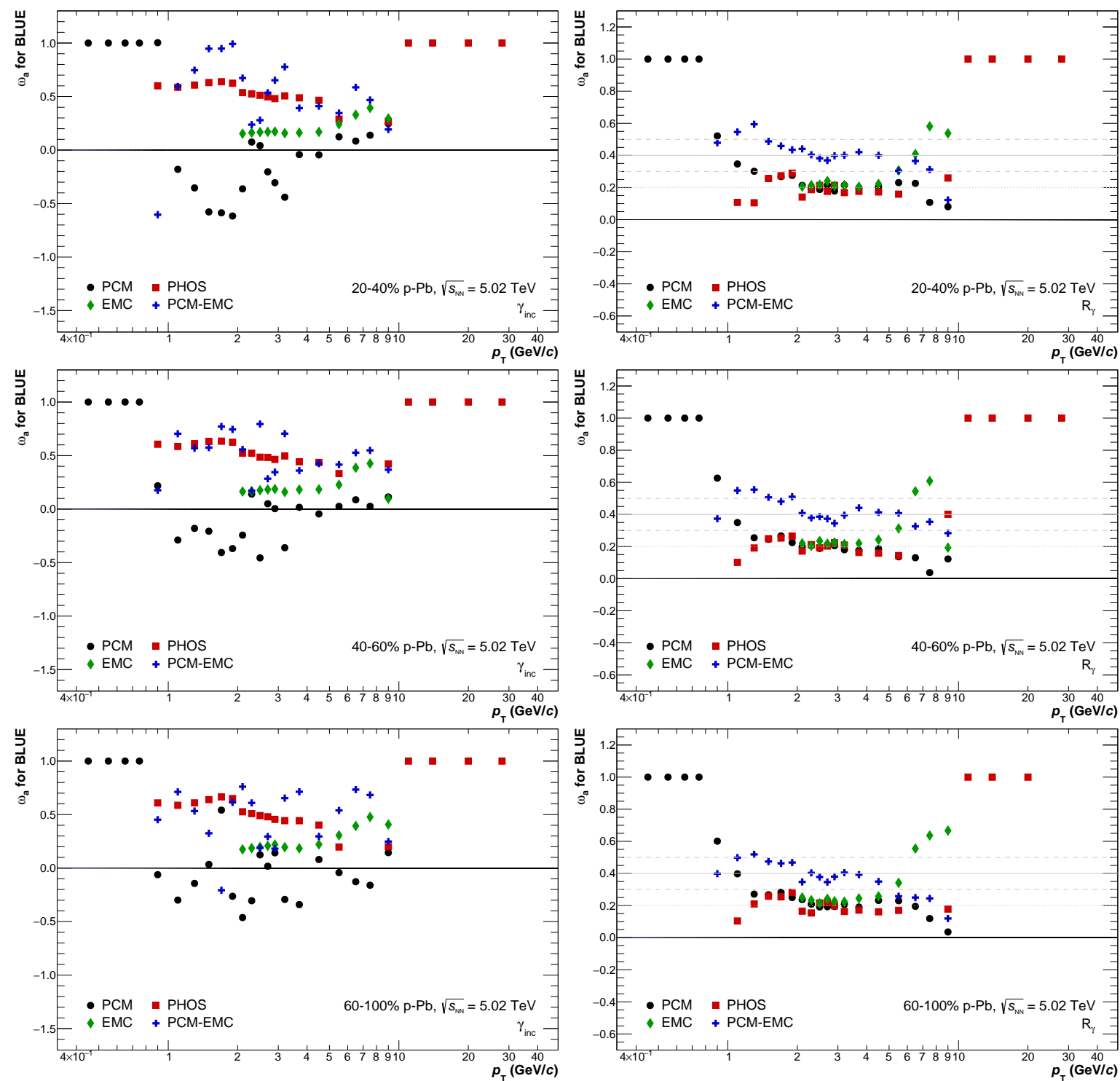

Figure A.18: Weights $\omega_{a}$ of the individual $\gamma_{\text {inv }}$ (left) and $R_{\gamma}$ (right) measurements according to the BLUE method for the 20-40\% (top), 40-60\% (middle) and 60-100\% (bottom) V0A multiplicity bin in $\mathrm{p}-\mathrm{Pb}$ collisions at $\sqrt{s_{\mathrm{NN}}}=5.02 \mathrm{TeV}$. 


\section{A.9.3 Relative uncertainties of combined spectra}
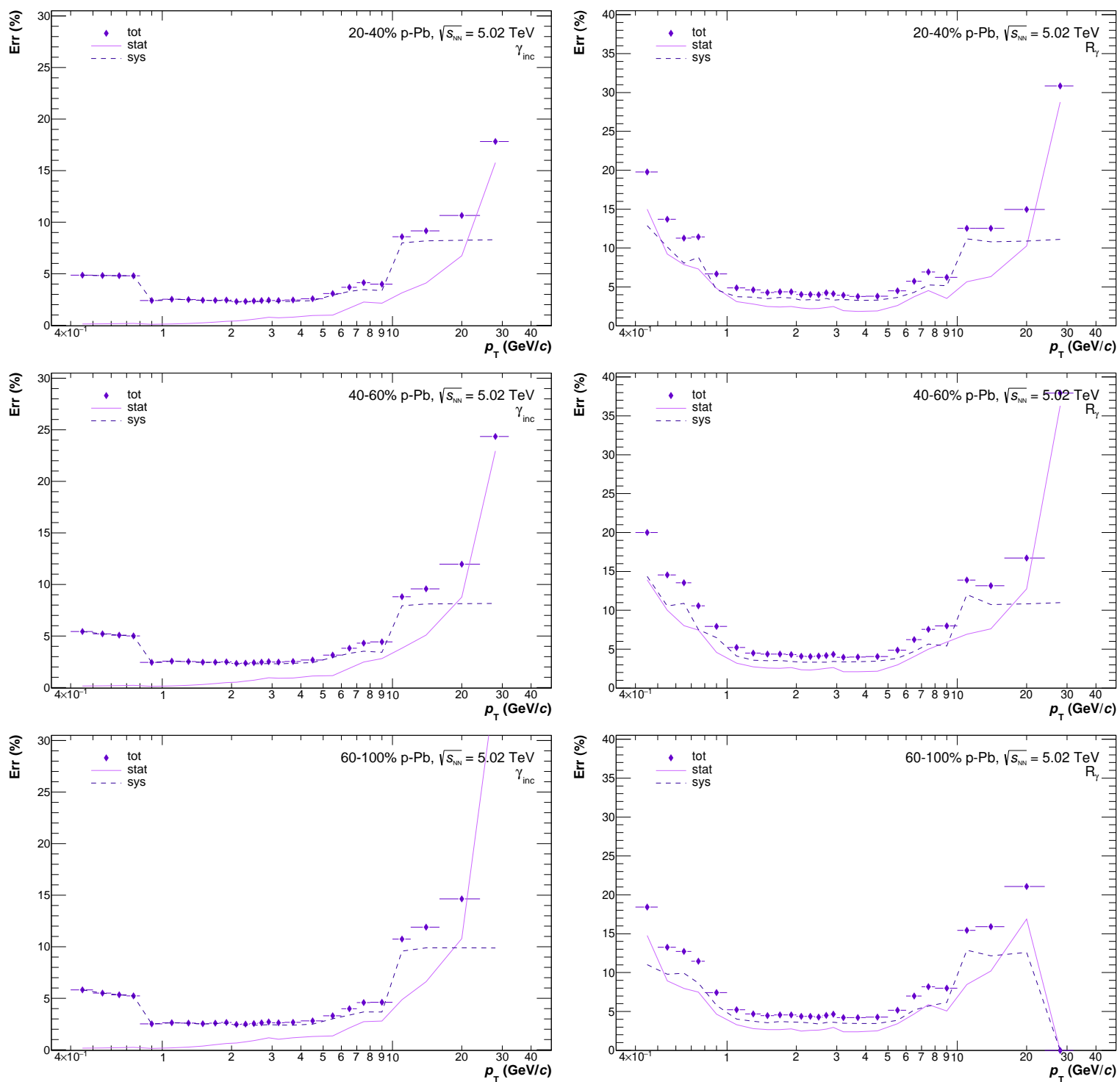

Figure A.19: Statistical, systematic and resulting total $p_{\mathrm{T}}$-dependent uncertainties of the inclusive photon (left) and $R_{\gamma}$ (right) measurements. 


\section{References}

[1] J. Chadwick, "Possible Existence of a Neutron", Nature 129 (1932)a 312.

[2] J. Chadwick, "The Existence of a Neutron", Proc. Roy. Soc. Lond. A 136 (1932)b, no. $830,692-708$.

[3] J. Thomson, "Cathode rays, the electrician, vol. 39, no. 104, also published in", in "Proceedings of the Royal Institution", vol. 30, pp. 1-14. 1897.

[4] E. Rutherford, "Collision of $\alpha$ particles with light atoms. IV. An anomalous effect in nitrogen", Phil. Mag. 90 (2010), no. sup1, 31-37.

[5] C. D. Anderson, "The Apparent Existence of Easily Deflectable Positives", Science 76 (1932) 238-239.

[6] C. M. G. Lattes, G. P. S. Occhialini, and C. F. Powell, "A determination of the ratio of the masses of - and -mesons by the method of grain-counting", Proceedings of the Physical Society 61 (1948), no. 2, 173-183.

[7] G. Rochester and C. Butler, "Evidence for the Existence of New Unstable Elementary Particles", Nature 160 (1947) 855-857.

[8] M. Gell-Mann, "The Eightfold Way: A Theory of strong interaction symmetry", 31961.

[9] S. Glashow, "Partial Symmetries of Weak Interactions", Nucl.Phys. 22 (1961) 579-588.

[10] A. Salam, "Weak and Electromagnetic Interactions", Conf.Proc. C680519 (1968)a $367-377$.

[11] A. Salam, "Fundamental Theory of Matter: A Survey of Results and Methods.", in "Contempory Physics: Trieste Symposium", vol. 19, p. 3. International Atomic Energy Agency, June 1968b.

[12] S. Weinberg, "A Model of Leptons", Phys.Rev.Lett. 19 (1967) 1264-1266.

[13] C. Burgess and G. Moore, "The standard model: A primer", 2007.

[14] P. W. Higgs, "Broken Symmetries and the Masses of Gauge Bosons", Physical Review Letters 13 (1964) 508-509.

[15] CMS Collaboration, S. Chatrchyan et al., "Observation of a new boson at a mass of $125 \mathrm{GeV}$ with the CMS experiment at the LHC", Phys.Lett. B716 (2012) 30-61, arXiv: 1207.7235 .

[16] ATLAS Collaboration, G. Aad et al., "Observation of a new particle in the search for the Standard Model Higgs boson with the ATLAS detector at the LHC", Phys.Lett. B716 (2012) 1-29, arXiv:1207.7214.

[17] F. Englert and R. Brout, "Broken Symmetry and the Mass of Gauge Vector Mesons", Phys.Rev.Lett. 13 (1964) 321-323.

[18] M. Thomson, "Modern particle physics", Cambridge University Press, New York, 2013. 
[19] P. D. Group and P. A. e. a. Zyla, "Review of Particle Physics", Progress of Theoretical and Experimental Physics 202008 (2020) https://academic.oup.com/ptep/article-pdf/2020/8/083C01/33653179/ptaa104.pdf, $083 \mathrm{C} 01$.

[20] I. de Física Corpuscular, "Lattice qcd, the numerical approach to the strong force". https://webific.ific.uv.es/web/en/content/ lattice-qcd-numerical-approach-strong-force

[21] J. Greensite, "An introduction to the confinement problem", 2011.

[22] D. H. Perkins, "Introduction to high energy physics; 4th ed.", Cambridge Univ. Press, Cambridge, 2000.

[23] J. C. Collins and M. Perry, "Superdense Matter: Neutrons Or Asymptotically Free Quarks?", Phys. Rev. Lett. 34 (1975) 1353.

[24] N. Cabibbo and G. Parisi, "Exponential Hadronic Spectrum and Quark Liberation", Phys.Lett. B59 (1975) 67-69.

[25] H. Satz, "Statistical mechanics of quarks and hadrons: Proceedings of an international symposium held at the university of bielefeld, f.r.g., august 24-31, 1980", North-Holland, 1981.

[26] M. Cheng et al., "The Transition temperature in QCD", Phys. Rev. D 74 (2006) 054507, hep-lat/0608013.

[27] R. Fries and B. Muller, "Heavy ions at LHC: Theoretical issues", Eur. Phys. J. C 34 (2004) S279-S285, nucl-th/0307043.

[28] Y. Aoki, S. Borsanyi, S. Durr, Z. Fodor, S. D. Katz, S. Krieg, and K. K. Szabo, "The QCD transition temperature: results with physical masses in the continuum limit II.", JHEP 06 (2009) 088, arXiv:0903.4155.

[29] F. Karsch, "Lattice QCD at high temperature and density", Lect. Notes Phys. 583 (2002) 209-249, hep-lat/0106019.

[30] F. Karsch, E. Laermann, and A. Peikert, "The Pressure in two flavor, (2+1)-flavor and three flavor QCD", Phys. Lett. B 478 (2000) 447-455, hep-lat/0002003.

[31] P. Braun-Munzinger, K. Redlich, and J. Stachel, "Particle production in heavy ion collisions", arXiv:nucl-th/0304013.

[32] D. H. Rischke, "The Quark gluon plasma in equilibrium", Prog. Part. Nucl. Phys. 52 (2004) 197-296, nucl-th/0305030.

[33] M. G. Alford, A. Schmitt, K. Rajagopal, and T. Schäfer, "Color superconductivity in dense quark matter", Rev. Mod. Phys. 80 (2008) 1455-1515, arXiv:0709.4635.

[34] J. Stachel and G. Young, "Relativistic heavy ion physics at CERN and BNL", Ann. Rev. Nucl. Part. Sci. 42 (1992) 537-597.

[35] H. Schmidt and J. Schukraft, "The Physics of ultrarelativistic heavy ion collisions", J. Phys. G 19 (1993) 1705-1796. 
[36] NA38 Collaboration, C. Baglin et al., "Transverse energy distributions in nucleus-nucleus collisions at 200-GeV/nucleon.", Phys. Lett. B 251 (1990) 472-476.

[37] M. L. Miller, K. Reygers, S. J. Sanders, and P. Steinberg, "Glauber modeling in high energy nuclear collisions", Ann. Rev. Nucl. Part. Sci. 57 (2007) 205-243, nucl-ex/0701025.

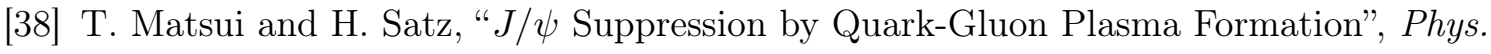
Lett. B 178 (1986) 416-422.

[39] ALICE Collaboration, J. Adam et al., "Centrality dependence of the charged-particle multiplicity density at midrapidity in Pb-Pb collisions at $\sqrt{s_{\mathrm{NN}}}=5.02 \mathrm{TeV}$ ", Phys. Rev. Lett. 116 (2016), no. 22, 222302, arXiv:1512.06104.

[40] W. Florkowski, "Basic phenomenology for relativistic heavy-ion collisions", Acta Phys. Polon. B 45 (2014), no. 12, 2329-2354, arXiv:1410.7904.

[41] W. Broniowski, M. Chojnacki, W. Florkowski, and A. Kisiel, "Uniform Description of Soft Observables in Heavy-Ion Collisions at $\mathrm{s}(\mathrm{NN})^{* *}(1 / 2)=200 \mathrm{GeV}^{* *} 2$ ", Phys. Rev. Lett. 101 (2008) 022301, arXiv:0801.4361.

[42] A. Bialas, M. Bleszynski, and W. Czyz, "Multiplicity Distributions in Nucleus-Nucleus Collisions at High-Energies", Nucl. Phys. B 111 (1976) 461-476.

[43] ALICE Collaboration, B. Abelev et al., "Centrality Dependence of Charged Particle Production at Large Transverse Momentum in $\mathrm{Pb}-\mathrm{Pb}$ Collisions at $\sqrt{s_{\mathrm{NN}}}=2.76 \mathrm{TeV}$ ", Physics Letters B 720 (2013), no. 1-3, 52 - 62, arXiv:1208.2711.

[44] PHENIX Collaboration, S. Adler et al., "Systematic studies of the centrality and $\sqrt{s_{\mathrm{NN}}}$ dependence of the $\mathrm{d} E_{\mathrm{T}} / \mathrm{d} \eta$ and $\mathrm{d} N_{\mathrm{ch}} / \mathrm{d} \eta$ in heavy ion collisions at mid-rapidity", Phys. Rev. C 71 (2005) 034908, nucl-ex/0409015, [Erratum: Phys.Rev.C 71, 049901 (2005)].

[45] PHOBOS Collaboration, B. Alver et al., "Phobos results on charged particle multiplicity and pseudorapidity distributions in $\mathrm{Au}+\mathrm{Au}, \mathrm{Cu}+\mathrm{Cu}, \mathrm{d}+\mathrm{Au}$, and $\mathrm{p}+\mathrm{p}$ collisions at ultra-relativistic energies", Phys. Rev. C 83 (2011) 024913, arXiv:1011.1940.

[46] J. Bjorken, "Highly Relativistic Nucleus-Nucleus Collisions: The Central Rapidity Region", Phys.Rev. D27 (1983) 140-151.

[47] F. Karsch and E. Laermann, "Thermodynamics and in medium hadron properties from lattice QCD", hep-lat/0305025.

[48] CMS Collaboration, S. Chatrchyan et al., "Measurement of the pseudorapidity and centrality dependence of the transverse energy density in $\mathrm{PbPb}$ collisions at $\sqrt{s_{\mathrm{NN}}}=2.76$ TeV", Phys. Rev. Lett. 109 (2012) 152303, arXiv:1205.2488.

[49] STAR Collaboration, H. Masui, "System size, energy and centrality dependence of strange hadron elliptic flow at STAR", J. Phys. G 36 (2009) 064047, arXiv:0903.0350.

[50] J.-Y. Ollitrault, "Anisotropy as a signature of transverse collective flow", Phys.Rev. D46 (1992) 229-245.

[51] U. W. Heinz, Early collective expansion: Relativistic hydrodynamics and the transport properties of QCD matter, vol. 23, p. 240. 2010. arXiv:0901.4355. 
[52] J.-Y. Ollitrault, "Anisotropy as a signature of transverse collective flow", Phys. Rev. D 46 (1992) 229-245.

[53] S. Voloshin and Y. Zhang, "Flow study in relativistic nuclear collisions by Fourier expansion of Azimuthal particle distributions", Z. Phys. C 70 (1996) 665-672, hep-ph/9407282.

[54] ALICE Collaboration, I. Selyuzhenkov, "Charged particle directed flow in $\mathrm{Pb}-\mathrm{Pb}$ collisions at $\sqrt{s_{\mathrm{NN}}}=2.76 \mathrm{TeV}$ measured with ALICE at the LHC", J.Phys. G38 (2011) 124167 , arXiv: 1106.5425 .

[55] G. Welke, M. Prakash, T. Kuo, S. Das Gupta, and C. Gale, "Azimuthal distributions in heavy ion collisions and the nuclear equation of state", Phys. Rev. C 38 (1988) 2101-2107.

[56] FOPI, A. Andronic, "Elliptic flow and equation of state in heavy ion collisions at SIS energies", Nucl. Phys. A 661 (1999) 333-336.

[57] ALICE Collaboration, J. Adam et al., "Multiplicity dependence of charged pion, kaon, and (anti)proton production at large transverse momentum in $\mathrm{p}-\mathrm{Pb}$ collisions at $\sqrt{s_{\mathrm{NN}}}=$ 5.02 TeV", Phys. Lett. B 760 (2016) 720-735, arXiv:1601.03658.

[58] M. Bombara, "Nuclear modification factor for $\xi$ and $\omega$ in p-pb collisions at $\sqrt{s_{\mathrm{NN}}}=5.02$ tev", 2016.

https://alice-notes.web.cern.ch/node/499

[59] CMS Collaboration, S. Chatrchyan et al., "Study of $W$ boson production in $\mathrm{PbPb}$ and $p p$ collisions at $\sqrt{s_{\mathrm{NN}}}=2.76 \mathrm{TeV}$ ", Phys. Lett. B $\mathbf{7 1 5}$ (2012) 66-87, arXiv:1205.6334.

[60] ALICE Collaboration, B. B. Abelev et al., "Production of charged pions, kaons and protons at large transverse momenta in pp and $\mathrm{Pb}-\mathrm{Pb}$ collisions at $\sqrt{s_{\mathrm{NN}}}=2.76 \mathrm{TeV}$ ", Phys. Lett. B736 (2014) 196-207, arXiv:1401.1250.

[61] ALICE Collaboration, B. B. Abelev et al., " $K_{S}^{0}$ and $\Lambda$ production in $\mathrm{Pb}-\mathrm{Pb}$ collisions at $\sqrt{s_{\mathrm{NN}}}=2.76 \mathrm{TeV} "$, Phys. Rev. Lett. 111 (2013) 222301, arXiv:1307.5530.

[62] ALICE Collaboration, J. Adam et al., "Transverse momentum dependence of D-meson production in $\mathrm{Pb}-\mathrm{Pb}$ collisions at $\sqrt{\mathrm{s}_{\mathrm{NN}}}=2.76 \mathrm{TeV}$ ", JHEP 03 (2016) 081, arXiv: 1509.06888.

[63] J. Cronin, H. J. Frisch, M. Shochet, J. Boymond, R. Mermod, P. Piroue, and R. L. Sumner, "Production of hadrons with large transverse momentum at 200, 300, and 400 GeV", Phys. Rev. D 11 (1975) 3105-3123.

[64] A. Krzywicki, J. Engels, B. Petersson, and U. Sukhatme, "Does a Nucleus Act Like a Gluon Filter?", Phys. Lett. B 85 (1979) 407-412.

[65] J.-w. Qiu and G. F. Sterman, "Power corrections in hadronic scattering. 1. Leading 1/Q**2 corrections to the Drell-Yan cross-section", Nucl. Phys. B 353 (1991) 105-136.

[66] BRAHMS Collaboration, R. Debbe, "High $\mathrm{p}(\mathrm{T})$ hadron spectra at high rapidity", $J$. Phys. G 30 (2004), no. 8, S759-S765, nucl-ex/0403052.

[67] I.-C. Arsene, I. Bearden, D. Beavis, S. Bekele, C. Besliu, B. Budick, H. Bøggild, C. Chasman, C. Christensen, P. Christiansen, R. Debbe, J. Gaardhøje, K. Hagel, H. Ito, A. Jipa, E. Johnson, F. Jundt, J. Jørdre, C. Jørgensen, and I. Zgura, "The brahms collaboration", Nuclear Physics A 77407 (2006) 938-938. 
[68] J. P. Blaizot, F. Gelis, and R. Venugopalan, "High-energy pA collisions in the color glass condensate approach. 1. Gluon production and the Cronin effect", Nucl. Phys. A $\mathbf{7 4 3}$ (2004) 13-56, hep-ph/0402256.

[69] H1and for the ZEUS, R. Placakyte, "Parton Distribution Functions", in "31st International Symposium on Physics In Collision". 11 2011. arXiv:1111.5452.

[70] E. Iancu and R. Venugopalan, The Color glass condensate and high-energy scattering in QCD, pp. 249-3363. 3 2003. hep-ph/0303204.

[71] NNPDF, R. D. Ball et al., "Parton distributions for the LHC Run II", JHEP 04 (2015) 040, arXiv: 1410.8849.

[72] A. Cooper-Sarkar, "What did HERA teach us about the structure of the proton?", $J$. Phys. G 39 (2012) 093001, arXiv:1206.0894.

[73] H1, ZEUS, H. Abramowicz et al., "Combination of measurements of inclusive deep inelastic $e^{ \pm} p$ scattering cross sections and QCD analysis of HERA data", Eur. Phys. J. C 75 (2015), no. 12,580 , arXiv: 1506.06042.

[74] Y. L. Dokshitzer, "Calculation of the Structure Functions for Deep Inelastic Scattering and e+ e- Annihilation by Perturbation Theory in Quantum Chromodynamics.", Sov. Phys. JETP 46 (1977) 641-653.

[75] V. Gribov and L. Lipatov, "Deep inelastic e p scattering in perturbation theory", Sov. J. Nucl. Phys. 15 (1972) 438-450.

[76] G. Altarelli and G. Parisi, "Asymptotic Freedom in Parton Language", Nucl. Phys. B 126 (1977) 298-318.

[77] K. Kovarik et al., "nCTEQ15 - Global analysis of nuclear parton distributions with uncertainties in the CTEQ framework", Phys. Rev. D 93 (2016), no. 8, 085037, arXiv: 1509.00792.

[78] A. Hendry, D. Lichtenberg, and E. Predazzi, "Ratio of the Nucleon Structure Function F2 for Heavy Nuclei and Free Nucleons", Phys. Lett. B 136 (1984) 433-436.

[79] European Muon, J. Aubert et al., "The ratio of the nucleon structure functions $F 2_{n}$ for iron and deuterium", Phys. Lett. B 123 (1983) 275-278.

[80] S. J. Brodsky and H. J. Lu, "Shadowing and Antishadowing of Nuclear Structure Functions", Phys. Rev. Lett. 64 (1990) 1342.

[81] X.-N. Wang and M. Gyulassy, "Gluon shadowing and jet quenching in A + A collisions at $\mathrm{s}^{* *}(1 / 2)=200-\mathrm{GeV} "$, Phys. Rev. Lett. 68 (1992) 1480-1483.

[82] M. Gyulassy and M. Plumer, "Jet Quenching in Dense Matter", Phys. Lett. B 243 (1990) 432-438.

[83] R. Baier, Y. L. Dokshitzer, A. H. Mueller, and D. Schiff, "Radiative energy loss of high-energy partons traversing an expanding QCD plasma", Phys. Rev. C 58 (1998) 1706-1713, hep-ph/9803473.

[84] R. Baier, Y. L. Dokshitzer, S. Peigne, and D. Schiff, "Induced gluon radiation in a QCD medium", Phys. Lett. B 345 (1995) 277-286, hep-ph/9411409. 
[85] P. Stankus, "Direct photon production in relativistic heavy-ion collisions", Ann. Rev. Nucl. Part. Sci. 55 (2005) 517-554.

[86] L. D. McLerran and T. Toimela, "Photon and Dilepton Emission from the Quark - Gluon Plasma: Some General Considerations", Phys. Rev. D 31 (1985) 545.

[87] J.-F. Paquet, C. Shen, G. S. Denicol, M. Luzum, B. Schenke, S. Jeon, and C. Gale, "Production of photons in relativistic heavy-ion collisions", Phys. Rev. C 93 (2016), no. 4, 044906, arXiv:1509.06738.

[88] F. Bock, "Measurement of Direct Photons and Neutral Mesons in Small Collisions Systems with the ALICE Experiment at the LHC", PhD thesis, U. Heidelberg (main), 2017.

[89] ALICE Collaboration, J. Adam et al., "Direct photon production in $\mathrm{Pb}-\mathrm{Pb}$ collisions at $\sqrt{s_{\mathrm{NN}}}=2.76 \mathrm{TeV} "$, Phys. Lett. B 754 (2016) 235-248, arXiv:1509.07324.

[90] PHENIX Collaboration, S. Adler et al., "Centrality dependence of direct photon production in $\sqrt{s_{\mathrm{NN}}}=200$ gev au + au collisions", Phys. Rev. Lett. 94 (2005) 232301, nucl-ex/0503003.

[91] WA98 Collaboration, M. Aggarwal et al., "Direct photon production in $158 \mathrm{~A}-\mathrm{GeV}$ $\mathrm{Pb}^{208}+\mathrm{Pb}^{208}$ collisions", Phys.Rev.C, 2000 arXiv:nucl-ex/0006007.

[92] P. Khandai, P. Shukla, and V. Singh, "Meson spectra and $m_{T}$ scaling in $p+p, d+\mathrm{Au}$, and $\mathrm{Au}+\mathrm{Au}$ collisions at $\sqrt{s_{\mathrm{NN}}}=200 \mathrm{GeV}$ ", Phys.Rev. C84 (2011) 054904, arXiv:1110.3929.

[93] STAR Collaboration, B. Abelev et al., "Inclusive $\pi^{0}, \eta$, and direct photon production at high transverse momentum in $p+p$ and $d+\mathrm{Au}$ collisions at $\sqrt{s_{\mathrm{NN}}}=200 \mathrm{GeV}$ ", Phys. Rev. C 81 (2010) 064904, arXiv:0912.3838.

[94] PHENIX Collaboration, A. Adare et al., "Observation of direct-photon collective flow in $\sqrt{s_{\mathrm{NN}}}=200 \mathrm{GeV} \mathrm{Au}+\mathrm{Au}$ collisions", Phys. Rev. Lett. 109 (2012) 122302, arXiv: 1105.4126 .

[95] PHENIX Collaboration, A. Adare et al., "Azimuthally anisotropic emission of low-momentum direct photons in $\mathrm{Au}+\mathrm{Au}$ collisions at $\sqrt{s_{N N}}=200 \mathrm{GeV}$ ", Phys. Rev. C 94 (2016), no. 6, 064901, arXiv: 1509.07758.

[96] ALICE Collaboration, S. Acharya et al., "Direct photon elliptic flow in $\mathrm{Pb}-\mathrm{Pb}$ collisions at $\sqrt{s_{\mathrm{NN}}}=2.76 \mathrm{TeV}$ ", Phys. Lett. B 789 (2019) 308-322, arXiv:1805.04403.

[97] R. Snellings, "Elliptic Flow: A Brief Review", New J.Phys. 13 (2011) 055008, arXiv:1102.3010.

[98] R. Chatterjee, H. Holopainen, I. Helenius, T. Renk, and K. J. Eskola, "Elliptic flow of thermal photons from event-by-event hydrodynamic model", Phys. Rev. C 88 (2013) 034901, arXiv: 1305.6443.

[99] C. Shen, U. W. Heinz, J.-F. Paquet, and C. Gale, "Thermal photons as a quark-gluon plasma thermometer reexamined", Phys. Rev. C 89 (2014), no. 4, 044910, arXiv: 1308.2440. 
[100] ALICE Collaboration, S. Acharya et al., "Direct photon production at low transverse momentum in proton-proton collisions at $\sqrt{\mathbf{s}}=\mathbf{2 . 7 6}$ and $8 \mathrm{TeV}$ ", Phys. Rev. C 99 (2019), no. 2, 024912, arXiv:1803.09857.

[101] ALICE Collaboration, N. Schmidt, "Direct photon production and flow at low transverse momenta in pp, p- $\mathrm{Pb}$ and $\mathrm{Pb}-\mathrm{Pb}$ collisions", PoS HardProbes2018 (2018) 183, arXiv: 1812.08104.

[102] PHENIX Collaboration, A. Adare et al., "Direct photon production in $d+\mathrm{Au}$ collisions at $\sqrt{s_{\mathrm{NN}}}=200 \mathrm{GeV}$ ", Phys. Rev. C 87 (2013) 054907, arXiv:1208.1234.

[103] T. L. H. C. Project, "Technical specification for the supply of three pole measuring machines for the lhc superconducting dipole magnet", 2000.

https://lhc-proj-qawg.web.cern.ch/LHCQAP/Instructions/Tecspecguide.htm

[104] e. Evans, L. and e. Bryant, P., "LHC Machine", JINST 3 (2008) S08001.

[105] ATLAS Collaboration, G. Aad et al., "The ATLAS Experiment at the CERN Large Hadron Collider", JINST 3 (2008) S08003.

[106] CMS Collaboration, S. Chatrchyan et al., "The CMS experiment at the CERN LHC", JINST 3 (2008) S08004.

[107] M. Bajko, F. Bertinelli, et al., "Report of the Task Force on the Incident of 19th September 2008 at the LHC", Tech. Rep. LHC-PROJECT-Report-1168.

CERN-LHC-PROJECT-Report-1168, CERN, 2009.

[108] R. Scrivens, M. Kronberger, D. Küchler, J. Lettry, C. Mastrostefano, O. Midttun, M. O'Neil, H. Pereira, and C. Schmitzer, "Overview of the status and developments on primary ion sources at CERN*", 2011.

[109] E. Boltezar, H. Haseroth, W. Pirkl, G. Plass, T. Sherwood, U. Tallgren, P. Tetu, D. Warner, and M. Weiss, "PERFORMANCE OF THE NEW CERN 50-MeV LINAC.", IEEE Trans. Nucl. Sci. 26 (1979)a 3674-3676.

[110] E. Boltezar, H. Haseroth, W. Pirkl, G. Plass, T. Sherwood, U. Tallgren, P. Tetu, D. Warner, and M. Weiss, "THE NEW CERN 50-MEV LINAC.", in "10th International Linear Accelerator Conference", pp. 66-77. 1 1979b.

[111] M. A. Hone, "The duoplasmatron ion source for the new CERN Linac preinjector", Tech. Rep. CERN-PS-LR-79-37, CERN, Geneva, 1979.

[112] P. Sortais, J. Bouly, N. Chauvin, J. Curdy, R. Geller, T. Lamy, P. Sole, and J. Vieux-Rochaz, "ECRIS as ion source and charge breeder", Nucl. Phys. A 701 (2002) $537-549$.

[113] N. Angert, M. P. Bourgarel, E. Brouzet, et al., "CERN heavy-ion facility design report", CERN, 1993.

[114] M. Chanel, "LEIR: The low energy ion ring at CERN", Nucl. Instrum. Methods Phys. Res., A 532 (2002), no. CERN-PS-2002-015-AE. CERN-AB-2003-086-ABP, 137-143. 9 p.

[115] K. Reich, "The CERN Proton Synchrotron Booster", IEEE Trans. Nucl. Sci. 16 (1969) 959-961. 
[116] "Report on the design study of a $300 \mathrm{GeV}$ proton synchrotron", CERN, Geneva, 1964. French version available.

[117] W. P. C. Mills, "The present performance of the SPS", IEEE Trans. Nucl. Sci. 26 (1979), no. CERN-SPS-AOP-79-9, 3176-3178. 3 p.

[118] "Design Report TeVatron 1 project", Tech. Rep. FERMILAB-DESIGN-1984-01, 1984.

[119] N. Doble, L. Gatignon, K. Hübner, and E. Wilson, The Super Proton Synchrotron (SPS): A Tale of Two Lives, vol. 27, pp. 135-177. 2017.

[120] D. Barney, "CMS Detector Slice", CMS Collection., 2016, unpublished. https://cds.cern.ch/record/2120661

[121] ATLAS TRT, V. A. Mitsou, "The ATLAS transition radiation tracker", in "8th International Conference on Advanced Technology and Particle Physics (ICATPP 2003): Astroparticle, Particle, Space Physics, Detectors and Medical Physics Applications", pp. 497-501. 11 2003. hep-ex/0311058.

[122] LHCb Collaboration, "LHCb technical design report: Reoptimized detector design and performance", 92003.

[123] LHCb Collaboration, J. Alves, A.Augusto et al., "The LHCb Detector at the LHC", JINST 3 (2008) S08005.

[124] LHCb Collaboration, R. Aaij et al., "LHCb Detector Performance", Int. J. Mod. Phys. A 30 (2015), no. 07, 1530022, arXiv:1412.6352.

[125] TOTEM Collaboration, G. Anelli et al., "The TOTEM experiment at the CERN Large Hadron Collider", JINST 3 (2008) S08007.

[126] J. Pinfold, "The MoEDAL Experiment at the LHC -a New Light on the Terascale Frontier", J. Phys. Conf. Ser. 631 (2015), no. 1, 012014.

[127] LHCf Collaboration, O. Adriani et al., "The LHCf detector at the CERN Large Hadron Collider", JINST 3 (2008) S08006.

[128] ALICE Collaboration, K. Aamodt et al., "The ALICE experiment at the CERN LHC", JINST 3 (2008) S08002.

[129] A. Collaboration, "Alice figure repository". https://alice-figure.web.cern.ch

[130] F. Wittgenstein, A. Herve, M. Feldmann, D. Luckey, and I. Vetlitsky, "CONSTRUCTION OF THE L3 MAGNET", in "11th International Conference on Magnet Technology (MT-11)", pp. 130-135. 81989.

[131] "Technical proposal: L3", Tech. Rep. CERN-LEPC-83-5. LEPC-P-4, 1983.

[132] ALICE TOF, A. Akindinov et al., "Particle identification with the ALICE TOF detector at very high particle multiplicity", Eur. Phys. J. C 32S1 (2004) 165-177.

[133] ALICE Collaboration, P. Kuijer, "The inner tracking system of the Alice experiment", Nucl. Instrum. Meth. A 530 (2004) 28-32. 
[134] ALICE Collaboration, "ALICE Inner Tracking System (ITS): Technical Design Report", CERN, Geneva, 1999.

[135] ALICE Collaboration, G. Dellacasa, L. Ramello, E. Scalas, M. Sitta, et al., "ALICE time projection chamber: Technical Design Report", CERN, Geneva, 2000.

[136] NA49 Collaboration, S. Afanasiev et al., "The NA49 large acceptance hadron detector", Nucl. Instrum. Meth. A 430 (1999) 210-244.

[137] ALICE Collaboration, B. B. Abelev et al., "Performance of the ALICE Experiment at the CERN LHC", Int. J. Mod. Phys. A29 (2014) 1430044, arXiv:1402.4476.

[138] ALICE collaboration, "First results of the ALICE detector performance at 13 TeV", 2015.

[139] H. Bethe, "Zur theorie des durchgangs schneller korpuskularstrahlen durch materie", Annalen der Physik 397 (1930), no. 3, 325-400, https://onlinelibrary.wiley.com/doi/pdf/10.1002/andp. 19303970303.

[140] P. Sigmund, "Particle penetration and radiation effects", Springer, 2008. ISBN: 978-3-540-72622-7, 012008.

[141] E. B. Podgorsak, "Radiation Physics for Medical Physicists; 2nd ed.", Springer, Berlin, Heidelberg, 2010.

[142] ALICE Collaboration, P. Cortese, "ALICE transition-radiation detector: Technical Design Report”, CERN, Geneva, 2001.

[143] V. Ginzburg and I. Frank, "Radiation of a uniformly moving electron due to its transition from one medium into another", J. Phys. (USSR) 9 (1945) 353-362.

[144] K. O. Schweda, "Space-charge distortion measurements and their calibration in the alice tpc", ALICE Analysis Note, unpublished.

https://aliceinfo.cern.ch/Notes/node/863

[145] ALICE Collaboration, G. Dellacasa et al., "ALICE technical design report of the time-of-flight system (TOF)", 22000.

[146] A. Akindinov et al., "Latest results on the performance of the multigap resistive plate chamber used for the ALICE TOF", Nucl. Instrum. Meth. A 533 (2004) 74-78.

[147] ALICE Collaboration, C. W. Fabjan et al., "ALICE: Physics performance report, volume II", J. Phys. G 32 (2006) 1295-2040.

[148] ALICE Collaboration, P. Cortese et al., "ALICE electromagnetic calorimeter technical design report", 92008.

[149] J. Allen et al., "ALICE DCal: An Addendum to the EMCal Technical Design Report Di-Jet and Hadron-Jet correlation measurements in ALICE", 62010.

[150] A. Collaboration, "Performance of the alice electromagnetic calorimeter", 2020, unpublished.

[151] ALICE Collaboration, G. Dellacasa et al., "ALICE technical design report of the photon spectrometer (PHOS)", 31999. 
[152] ALICE PHOS calorimeter, D. Aleksandrov et al., "A high resolution electromagnetic calorimeter based on lead-tungstate crystals", Nucl. Instrum. Meth. A $\mathbf{5 5 0}$ (2005) $169-184$.

[153] ALICE Collaboration, S. Acharya et al., "Calibration of the photon spectrometer PHOS of the ALICE experiment", JINST 14 (2019), no. 05, P05025, arXiv: 1902.06145.

[154] D. Wang et al., "Level-0 trigger algorithms for the ALICE PHOS detector", Nucl. Instrum. Meth. A 629 (2011) 80-86.

[155] ALICE Collaboration, S. Beole et al., "ALICE technical design report: Detector for high momentum PID", 81998.

[156] ALICE Collaboration, G. Dellacasa et al., "ALICE technical design report of the zero degree calorimeter (ZDC)", 3 1999a.

[157] ALICE Collaboration, G. Dellacasa et al., "ALICE technical design report: Photon multiplicity detector (PMD)", 9 1999b.

[158] ALICE Collaboration, P. Cortese et al., "ALICE technical design report on forward detectors: FMD, T0 and V0", 92004.

[159] ALICE Collaboration, E. Abbas et al., "Performance of the ALICE VZERO system", JINST 8 (2013) P10016, arXiv: 1306.3130.

[160] J. Conrad, J. Contreras, and C. Jorgensen, "Minimum bias triggers in proton-proton collisions with VZERO and pixel detectors", 2005.

[161] ALICE Collaboration, B. B. Abelev et al., "Measurement of visible cross sections in proton-lead collisions at $\sqrt{s_{\mathrm{NN}}}=5.02 \mathrm{TeV}$ in van der Meer scans with the ALICE detector", JINST 9 (2014), no. 11, P11003, arXiv:1405.1849.

[162] F. R. et al, "root-project/root: v6.18/02", 2019. https://doi.org/10.5281/zenodo. 3895860

[163] G. Van Rossum and F. L. Drake, "Python 3 reference manual", CreateSpace, Scotts Valley, CA, 2009.

[164] ALICE Collaboration, "Aliroot-software". a. https://github.com/alisw/AliRoot

[165] "Aliphysics-software". b. https://github.com/alisw/AliPhysics

[166] T. Sjöstrand, S. Ask, J. R. Christiansen, R. Corke, N. Desai, P. Ilten, S. Mrenna, S. Prestel, C. O. Rasmussen, and P. Z. Skands, "An Introduction to PYTHIA 8.2", Comput. Phys. Commun. 191 (2015) 159-177, arXiv:1410.3012.

[167] P. Skands, S. Carrazza, and J. Rojo, "Tuning PYTHIA 8.1: the Monash 2013 Tune", Eur. Phys. J. C74 (2014), no. 8, 3024, arXiv:1404.5630.

[168] R. Engel, "Phojet manual", University of Siegen preprint, 1995 95-05.

[169] S. Roesler, R. Engel, and J. Ranft, "The Monte Carlo event generator DPMJET-III", in "International Conference on Advanced Monte Carlo for Radiation Physics, Particle Transport Simulation and Applications (MC 2000)", pp. 1033-1038. 122000. hep-ph/0012252. 
[170] J. Ranft, "DPMJET version II.5: Sampling of hadron hadron, hadron - nucleus and nucleus-nucleus interactions at accelerator and cosmic ray energies according to the two component dual parton model: Code manual", hep-ph/9911232.

[171] J. Ranft, "The Dual parton model at cosmic ray energies", Phys. Rev. D 51 (1995) 64-84.

[172] T. Pierog, I. Karpenko, J. Katzy, E. Yatsenko, and K. Werner, "EPOS LHC: Test of collective hadronization with data measured at the CERN Large Hadron Collider", Phys. Rev. C 92 (2015), no. 3, 034906, arXiv:1306.0121.

[173] R. Brun, F. Bruyant, M. Maire, A. McPherson, and P. Zanarini, "GEANT3", 1987.

[174] GEANT4, S. Agostinelli et al., "GEANT4-a simulation toolkit", Nucl. Instrum. Meth. A 506 (2003) 250-303.

[175] G. Battistoni et al., "Overview of the FLUKA code", Annals Nucl. Energy 82 (2015) $10-18$.

[176] R. Frühwirth, "Application of kalman filtering to track and vertex fitting", Nuclear Instruments and Methods in Physics Research Section A: Accelerators, Spectrometers, Detectors and Associated Equipment 262 (1987), no. 2-3, $444-450$.

[177] S. Gorbunov and I. Kisel, "Reconstruction of decayed particles based on the kalman filter". http://www.gsi.documents/DOC-2007-May-14-1.pdf

[178] ALICE Collaboration, "ALICE luminosity determination for pp collisions at $\sqrt{s}=8$ TeV", 2017.

[179] ALICE Collaboration, "ALICE luminosity determination for $\mathrm{p}-\mathrm{Pb}$ collisions at $\sqrt{s_{\mathrm{NN}}}=8.16 \mathrm{TeV} ", 2018$.

[180] M. Abramowitz and I. A. Stegun, "Handbook of mathematical functions with formulas, graphs, and mathematical tables", Dover, New York, ninth dover printing, tenth gpo printing ed., 1964.

[181] T. Sjostrand and P. Z. Skands, "Multiple interactions and the structure of beam remnants", JHEP 03 (2004) 053, hep-ph/0402078.

[182] S. Argyropoulos and T. Sjöstrand, "Effects of color reconnection on $t \bar{t}$ final states at the LHC", JHEP 11 (2014) 043, arXiv:1407.6653.

[183] B. Andersson, G. Gustafson, G. Ingelman, and T. Sjostrand, "Parton Fragmentation and String Dynamics", Phys. Rept. 97 (1983) 31-145.

[184] Particle Data Group, M. Tanabashi et al., "Review of Particle Physics", Phys. Rev. D 98 (2018), no. 3, 030001.

[185] R. Engel, J. Ranft, and S. Roesler, "Hard diffraction in hadron hadron interactions and in photoproduction", Phys.Rev. D52 (1995) 1459-1468, arXiv:hep-ph/9502319.

[186] A. Capella, U. Sukhatme, C.-I. Tan, and J. Tran Thanh Van, "Dual parton model", Phys.Rept. 236 (1994) 225-329.

[187] D. Armen Buniatian, "Large Transverse Energies and Jets in Hard Photoproduction at HERA", 1995. 
[188] P. Aurenche, F. W. Bopp, A. Capella, J. Kwiecinski, M. Maire, J. Ranft, and J. T. Thanh Van, "Multiparticle production in a two-component dual parton model", Phys. Rev. D 45 (1992) 92-105.

[189] S. Roesler, R. Engel, and J. Ranft, "Photoproduction off nuclei: Particle and jet production", Phys. Rev. D 57 (1998) 2889-2902.

[190] S. Shmakov, V. Uzhinsky, and A. Zadorozhnyi, "DIAGEN: GENERATOR OF INELASTIC NUCLEUS-NUCLEUS INTERACTION DIAGRAMS", 101988.

[191] H. Drescher, M. Hladik, S. Ostapchenko, T. Pierog, and K. Werner, "Parton based Gribov-Regge theory", Phys. Rept. 350 (2001) 93-289, hep-ph/0007198.

[192] ALICE EMCal, U. Abeysekara et al., "ALICE EMCal Physics Performance Report", arXiv: 1008.0413.

[193] F. Bock, "ALICE Capabilities for Studying Photon Physics with the Conversion Method at LHC Energies", bachelor's thesis, University of Heidelberg, June 2010.

http://www.physi.uni-heidelberg.de//Publications/Bock-Bachelorthesis.pdf

[194] K. Koch, "Measurement of $\pi^{0}$ and $\eta$ mesons with photon conversions in alice in proton-proton collisions at $\sqrt{s}=0.9,2.76,7$ tev", PhD thesis, University of Heidelberg, February 2012.

http://www.ub.uni-heidelberg.de/archiv/13113

[195] ALICE Collaboration, S. Acharya et al., "Production of $\pi^{0}$ and $\eta$ mesons up to high transverse momentum in pp collisions at $2.76 \mathrm{TeV}$ ", Eur. Phys. J. C 77 (2017), no. 5, 339, arXiv: 1702.00917.

[196] ALICE Collaboration, S. Acharya et al., " $\pi^{0}$ and $\eta$ meson production in proton-proton collisions at $\sqrt{s}=8 \mathrm{TeV}$ ", Eur. Phys. J. C 78 (2018), no. 3, 263, arXiv:1708.08745.

[197] ALICE Collaboration, B. B. Abelev et al., "Neutral pion production at midrapidity in pp and $\mathrm{Pb}-\mathrm{Pb}$ collisions at $\sqrt{s_{\mathrm{NN}}}=2.76 \mathrm{TeV}$ ", arXiv:1405.3794.

[198] J. Podolanski and R. Armenteros, "Analysis of v-events", Phil. Mag. 7 (1954).

[199] G. C. Balbastre, "Study and emulation of cell energy cross-talk in emcal", 2018, unpublished.

https://alice-notes.web.cern.ch/node/837

[200] ALICE EMCal, J. Allen et al., "Performance of prototypes for the ALICE electromagnetic calorimeter", Nucl. Instrum. Meth. A 615 (2010) 6-13, arXiv:0912. 2005.

[201] T. M. Carter, "ALICE EMCal Reconstructable Energy Non-Linearity From Test Beam Monte Carlo", 2017.

[202] M. Fasel, "Jet substructure in pp collisions at $\sqrt{s}=13$ tev", 2019.

[203] S. Yano, "Measurement of pi0 production in pp collisions at 8 tev with phos", ALICE Analysis Note, unpublished.

https://aliceinfo.cern.ch/Notes/node/385

[204] D. Y. Peresunko, "Measurement of neutral mesons in p-pb collisions at $\sqrt{s_{\mathrm{NN}}}=8.16$ tev with the phos detector", 2020.

https://alice-notes.web.cern.ch/node/1064 
[205] G. Kopylov, "Like particle correlations as a tool to study the multiple production mechanism", Phys. Lett. B 50 (1974) 472-474.

[206] ALICE Collaboration, B. Abelev et al., "Neutral pion and $\eta$ meson production in proton-proton collisions at $\sqrt{s}=0.9 \mathrm{TeV}$ and $\sqrt{s}=7 \mathrm{TeV}$ ", Phys. Lett. B 717 (2012) 162-172, arXiv:1205.5724.

[207] S. Pisano, "Production of pions, kaons and protons in p-pb collisions at 8.16 tev", 2019. https://alice-notes.web.cern.ch/node/769

[208] T. Sjöstrand, S. Mrenna, and P. Z. Skands, "PYTHIA 6.4 Physics and Manual", JHEP 0605 (2006) 026, arXiv:hep-ph/0603175.

[209] A. Valassi and R. Chierici, "Information and treatment of unknown correlations in the combination of measurements using the BLUE method", Eur. Phys. J. C 74 (2014) 2717, arXiv: 1307.4003.

[210] L. Lyons, D. Gibaut, and P. Clifford, "How to Combine Correlated Estimates of a Single Physical Quantity", Nucl. Instrum. Meth. A270 (1988) 110.

[211] S. Alioli, P. Nason, C. Oleari, and E. Re, "NLO vector-boson production matched with shower in POWHEG", JHEP 07 (2008) 060, arXiv:0805.4802.

[212] F. Bock, "Neutral Pion and Eta Meson Production in pp and $\mathrm{Pb}-\mathrm{Pb}$ Collisions at the LHC with the ALICE Detector", Master's thesis, University Heidelberg, December 2012. http://www.physi.uni-heidelberg.de//Publications/Bock-Masterthesis.pdf

[213] ALICE Collaboration, K. Koch, " $\pi^{0}$ and $\eta$ measurement with photon conversions in ALICE in proton-proton collisions at $\sqrt{s}=7 \mathrm{TeV}$ ", Nucl.Phys. A855 (2011) 281-284, arXiv: 1103.2217.

[214] G. D. Lafferty and T. R. Wyatt, "Where to stick your data points: The treatment of measurements within wide bins", Nucl.Instrum.Meth. A355 (1995) 541-547.

[215] C. Tsallis, "Possible Generalization of Boltzmann-Gibbs Statistics", J. Statist. Phys. 52 (1988) 479-487.

[216] A. Bylinkin and M. Ryskin, "Secondary hadron distributions in two component model", Phys. Rev. D 90 (2014), no. 1, 017501, arXiv:1404.4739.

[217] A. Bylinkin and A. Rostovtsev, "Role of quarks in hadroproduction in high energy collisions", Nucl. Phys. B 888 (2014) 65-74, arXiv:1404.7302.

[218] A. Bylinkin, N. S. Chernyavskaya, and A. A. Rostovtsev, "Predictions on the transverse momentum spectra for charged particle production at LHC-energies from a two component model", Eur. Phys. J. C75 (2015), no. 4, 166, arXiv:1501.05235.

[219] R. Hagedorn, "Multiplicities, $p_{T}$ Distributions and the Expected Hadron $\rightarrow$ Quark Gluon Phase Transition", Riv. Nuovo Cim. 6N10 (1983) 1-50.

[220] T. Sjostrand, S. Mrenna, and P. Z. Skands, "A Brief Introduction to PYTHIA 8.1", Comput. Phys. Commun. 178 (2008) 852-867, arXiv:0710.3820.

[221] H.-L. Lai, M. Guzzi, J. Huston, Z. Li, P. M. Nadolsky, J. Pumplin, and C.-P. Yuan, "New parton distributions for collider physics", Phys. Rev. D 82 (2010) 074024, arXiv: 1007.2241. 
[222] D. de Florian, R. Sassot, M. Epele, R. J. Hernández-Pinto, and M. Stratmann, "Parton-to-Pion Fragmentation Reloaded", Phys. Rev. D91 (2015), no. 1, 014035, arXiv: 1410.6027.

[223] C. A. Aidala, F. Ellinghaus, R. Sassot, J. P. Seele, and M. Stratmann, "Global Analysis of Fragmentation Functions for Eta Mesons", Phys. Rev. D83 (2011) 034002, arXiv: 1009.6145.

[224] W. Tung, H. Lai, A. Belyaev, J. Pumplin, D. Stump, and C.-P. Yuan, "Heavy Quark Mass Effects in Deep Inelastic Scattering and Global QCD Analysis", JHEP 02 (2007) 053, hep-ph/0611254.

[225] K. J. Eskola, P. Paakkinen, H. Paukkunen, and C. A. Salgado, "EPPS16: Nuclear parton distributions with LHC data", Eur. Phys. J. C 77 (2017), no. 3, 163, arXiv:1612.05741.

[226] B. Jager, A. Schafer, M. Stratmann, and W. Vogelsang, "Next-to-leading order QCD corrections to high $\mathrm{p}(\mathrm{T})$ pion production in longitudinally polarized pp collisions", Phys. Rev. D 67 (2003) 054005, hep-ph/0211007.

[227] PHENIX Collaboration, A. Adare et al., "Measurement of neutral mesons in $\mathrm{p}+\mathrm{p}$ collisions at $\sqrt{s}=200 \mathrm{GeV}$ and scaling properties of hadron production", Phys. Rev. D $\mathbf{8 3}$ (2011) 052004, arXiv:1005.3674.

[228] PHENIX Collaboration, A. Adare et al., "Neutral pion production with respect to centrality and reaction plane in $\mathrm{Au}+\mathrm{Au}$ collisions at $\sqrt{s_{\mathrm{NN}}}=200 \mathrm{GeV}$ ", arXiv: 1208.2254.

[229] PHENIX Collaboration, A. Adare et al., "Suppression pattern of neutral pions at high transverse momentum in $\mathrm{Au}+\mathrm{Au}$ collisions at $\sqrt{s_{\mathrm{NN}}}=200 \mathrm{GeV}$ and constraints on medium transport coefficients", Phys. Rev. Lett. 101 (2008) 232301, arXiv:0801.4020.

[230] PHENIX Collaboration, S. Adler et al., "High transverse momentum $\eta$ meson production in $p^{+} p, d^{+} \mathrm{Au}$ and $\mathrm{Au}+\mathrm{Au}$ collisions at $\sqrt{s_{\mathrm{NN}}}=200 \mathrm{GeV}$ ", Phys.Rev. C75 (2007) 024909, arXiv:nucl-ex/0611006.

[231] J. Adams, C. Adler, M. Aggarwal, Z. Ahammed, J. Amonett, B. Anderson, M. Anderson, D. Arkhipkin, G. Averichev, S. Badyal, et al., "Photon and neutral pion production in $\mathrm{Au}+\mathrm{Au}$ collisions at $\sqrt{s_{\mathrm{NN}}}=130 \mathrm{GeV}$ ", Physical Review C 70 (2004), no. 4, 44902.

[232] ALICE Collaboration, S. Acharya et al., "Neutral pion and $\eta$ meson production in $\mathrm{p}-\mathrm{Pb}$ collisions at $\sqrt{s_{\mathrm{NN}}}=5.02 \mathrm{TeV}$ ", Eur. Phys. J. C 78 (2018), no. 8, 624, arXiv: 1801.07051.

[233] D. de Florian, R. Sassot, and M. Stratmann, "Global analysis of fragmentation functions for pions and kaons and their uncertainties", Phys.Rev. D75 (2007) 114010, arXiv: hep-ph/0703242.

[234] L. Altenkämper, F. Bock, C. Loizides, and N. Schmidt, "Applicability of transverse mass scaling in hadronic collisions at energies available at the CERN Large Hadron Collider", Phys. Rev. C 96 (2017), no. 6, 064907, arXiv:1710.01933.

[235] R. Hagedorn, "Statistical thermodynamics of strong interactions at high-energies", Nuovo Cim. Suppl. 3 (1965) 147-186. 
[236] British-Scandinavian, B. Alper et al., "The Production of Charged Particles with High Transverse Momentum in Proton-Proton Collisions at the CERN ISR", Nucl. Phys. B $\mathbf{8 7}$ (1975) 19.

[237] M. Bourquin and J.-M. Gaillard, "A Simple Phenomenological Description of Hadron Production", Nucl. Phys. B 114 (1976) 334-364.

[238] STAR Collaboration, B. Abelev et al., "Strange particle production in $\mathrm{p}+\mathrm{p}$ collisions at s** $(1 / 2)=200-\mathrm{GeV}^{*}$, Phys. Rev. C 75 (2007) 064901, nucl-ex/0607033.

[239] P. Khandai, P. Sett, P. Shukla, and V. Singh, "Transverse Mass Spectra and Scaling of Hadrons at RHIC and LHC Energies", arXiv:1205.0648.

[240] Axial Field Spectrometer, T. Akesson et al., "Production of $\pi^{0}$ and Eta0 at 11-degrees in $p p$ Collisions at $\sqrt{s}=63-\mathrm{GeV} "$, Z. Phys. C18 (1983) 5.

[241] Axial Field Spectrometer, T. Akesson et al., "Inclusive $\eta$ Production at Low Transverse Momentum in 63-GeV pp Collisions at the CERN Intersecting Storage Rings", Phys. Lett. B178 (1986) 447.

[242] Axial Field Spectrometer, T. Akesson et al., "A comparison of direct photon, pi0, and eta production in p anti-p and pp interactions at the CERN ISR", Phys. Lett. 158B (1985) 282-288.

[243] C. Kourkoumelis et al., "Inclusive $\pi^{0}$ Production at Very Large $p_{T}$ at the ISR", Phys. Lett. 84B (1979)a 271-276.

[244] C. Kourkoumelis et al., "Inclusive $\eta$ Production at High $p_{T}$ at the ISR", Phys. Lett. 84B (1979)b 277-280.

[245] E. Amaldi et al., "INCLUSIVE ETA PRODUCTION IN P P COLLISION AT ISR ENERGIES", Nucl. Phys. B158 (1979) 1-10.

[246] CERN-Lausanne-Michigan-Rockefeller, UA6, J. Antille et al., "A Measurement of the Inclusive $\pi^{0}$ and $\eta$ Production Cross-sections at High $p_{T}$ in $p \bar{p}$ and $p p$ Collisions at $\sqrt{s}=24.3-\mathrm{GeV}^{\prime}$, Phys. Lett. B194 (1987) 568.

[247] UA2, Bern-CERN-Copenhagen-Orsay-Pavia-Saclay, M. Banner et al., "Inclusive Particle Production in the Transverse Momentum Range Between 0.25-GeV/c and $40-\mathrm{GeV} / c$ at the CERN S pp S Collider", Z. Phys. C27 (1985) 329.

[248] WA70 Collaboration, M. Bonesini et al., "High Transverse Momentum $\eta$ Production in $\pi^{-} p, \pi^{+} p$ and $p p$ Interactions at $280-\{\mathrm{GeV}\} / c^{\prime \prime}$, Z. Phys. C 42 (1989) 527.

[249] M. Aguilar-Benitez et al., "Inclusive particle production in $400-\mathrm{GeV} /$ c p p interactions", Z. Phys. C50 (1991) 405-426.

[250] G. J. Donaldson et al., "Inclusive eta Production at Large Transverse Momenta", Phys. Rev. Lett. 40 (1978) 684.

[251] Fermilab E706, L. Apanasevich et al., "Production of pi0 and eta mesons at large transverse momenta in pp and $\mathrm{p}$ Be interactions at $530-\mathrm{GeV} / c$ and $800-\mathrm{GeV} / c "$, Phys. Rev. D68 (2003) 052001, arXiv:hep-ex/0204031.

[252] G. Agakishiev et al., "Neutral meson production in p Be and p Au collisions at 450-GeV beam energy", Eur. Phys. J. C4 (1998) 249-257. 
[253] J. Povlis et al., "Nuclear Enhancement of pi0 and eta mesons Produced at Large Transverse Momenta", Phys. Rev. Lett. 51 (1983) 967.

[254] Fermilab E706 Collaboration, G. Alverson et al., "Production of direct photons and neutral mesons at large transverse momenta by $\pi^{-}$and $p$ beams at $500-\mathrm{GeV} / c$ ", Phys. Rev. D48 (1993) 5-28.

[255] Fermilab E706, L. Apanasevich et al., "Production of pi0 and eta mesons at large transverse momenta in pi- $\mathrm{p}$ and pi- Be interactions at $515-\mathrm{GeV} / c^{\prime \prime}$, Phys. Rev. D69 (2004) 032003, arXiv:hep-ex/0308022.

[256] ALICE Collaboration, M. Sas, "Light neutral meson production in heavy ion collisions with ALICE in the era of precision physics at the LHC", arXiv:2008.02036.

[257] ALICE Collaboration, S. Acharya et al., "Transverse momentum spectra and nuclear modification factors of charged particles in $\mathrm{pp}, \mathrm{p}-\mathrm{Pb}$ and $\mathrm{Pb}-\mathrm{Pb}$ collisions at the LHC", JHEP 11 (2018) 013, arXiv:1802.09145.

[258] CMS Collaboration, V. Khachatryan et al., "Charged-particle nuclear modification factors in $\mathrm{PbPb}$ and $\mathrm{p}-\mathrm{Pb}$ collisions at $\sqrt{s_{\mathrm{NN}}}=5.02 \mathrm{TeV}^{\prime}$, JHEP 04 (2017) 039, arXiv: 1611.01664.

[259] I. Ravasenga, "Multiplicity and centre-of-mass energy dependence of light-flavor hadron production in pp, p-Pb, and $\mathrm{Pb}-\mathrm{Pb}$ collisions with ALICE", PoS EPS-HEP2019 (2020) 290.

[260] J. Gao, M. Guzzi, J. Huston, H.-L. Lai, Z. Li, P. Nadolsky, J. Pumplin, D. Stump, and C.-P. Yuan, "CT10 next-to-next-to-leading order global analysis of QCD", Phys. Rev. D 89 (2014), no. 3, 033009, arXiv:1302.6246.

[261] M. Guzzi, P. Nadolsky, E. Berger, H.-L. Lai, F. Olness, and C.-P. Yuan, "CT10 parton distributions and other developments in the global QCD analysis", arXiv:1101.0561.

[262] T. Lappi and H. Mäntysaari, "Single inclusive particle production at high energy from HERA data to proton-nucleus collisions", Phys. Rev. D 88 (2013) 114020, arXiv:1309.6963.

[263] I. Balitsky, "Operator expansion for high-energy scattering", Nucl. Phys. B 463 (1996) 99-160, hep-ph/9509348.

[264] Y. V. Kovchegov, "Small x F(2) structure function of a nucleus including multiple pomeron exchanges", Phys. Rev. D 60 (1999) 034008, hep-ph/9901281.

[265] F. Arleo, F. Cougoulic, and S. Peigné, "Fully coherent energy loss effects on light hadron production in pA collisions", JHEP 09 (2020) 190, arXiv:2003.06337.

[266] J. Bjorken and S. J. Brodsky, "Statistical Model for electron-Positron Annihilation Into Hadrons", Phys. Rev. D 1 (1970) 1416-1420.

[267] I. W. Stewart, F. J. Tackmann, and W. J. Waalewijn, "N-Jettiness: An Inclusive Event Shape to Veto Jets", Phys. Rev. Lett. 105 (2010) 092002, arXiv:1004.2489.

[268] F. Bock, "Measurement of direct photons in p-pb collisions at $\sqrt{s_{\mathrm{NN}}}=5.023$ tev using the pcm, emc and pcm-emc reconstruction techniques", 2018.

https://alice-notes.web.cern.ch/node/813 
[269] D. Peresunko, "Multiplicity dependence of direct photon production in p-pb collisions at 5.02 tev with alice phos", 2018.

https://alice-notes.web.cern.ch/node/857

[270] F. Bock, "Combination of different neutral meson measurements in p-pb collisions at 5 tev - centrality dependent", 2018.

https://alice-notes.web.cern.ch/node/858

[271] ALICE Collaboration, J. Adam et al., "Production of $\mathrm{K}^{*}(892)^{0}$ and $\phi(1020)$ in $\mathrm{p}-\mathrm{Pb}$ collisions at $\sqrt{s_{\mathrm{NN}}}=5.02 \mathrm{TeV}$ ", Eur. Phys. J. C 76 (2016)a, no. 5, 245, arXiv: 1601.07868 .

[272] ALICE Collaboration, J. Adam et al., "Multi-strange baryon production in $\mathrm{p}-\mathrm{Pb}$ collisions at $\sqrt{s_{\mathrm{NN}}}=5.02 \mathrm{TeV}$ ", Phys. Lett. B 758 (2016)b 389-401, arXiv:1512.07227.

[273] ALICE Collaboration, B. B. Abelev et al., "Multiplicity Dependence of Pion, Kaon, Proton and Lambda Production in p-Pb Collisions at $\sqrt{s_{\mathrm{NN}}}=5.02 \mathrm{TeV}$ ", Phys. Lett. B 728 (2014) 25-38, arXiv:1307.6796.

[274] N. S. Annika Passfeld, "Multiplicity dependent neutral meson measurements via photon conversions in p-pb collisions at $\sqrt{s_{\mathrm{NN}}}=5.02$ tev", 2018 .

https://alice-notes.web.cern.ch/node/856

[275] M. Sas, "Centrality dependent neutral meson measurements with emc, pcm-emc, and pcm-phos in alice in p-pb collisions(run1) at $\sqrt{s}=5.023$ tev", 2018.

https://alice-notes.web.cern.ch/node/830

[276] E. Schnedermann, J. Sollfrank, and U. W. Heinz, "Thermal phenomenology of hadrons from 200-A/GeV S+S collisions", Phys. Rev. C 48 (1993) 2462-2475, nucl-th/9307020.

[277] G. D'Agostini, "A multidimensional unfolding method based on bayes' theorem", Nuclear Instruments and Methods in Physics Research Section A: Accelerators, Spectrometers, Detectors and Associated Equipment 362 (1995), no. 2, 487 - 498.

[278] A. Dale, "Chapter 15 - thomas bayes, an essay towards solving a problem in the doctrine of chances (1764)", in "Landmark Writings in Western Mathematics 1640-1940",

I. Grattan-Guinness, R. Cooke, L. Corry, P. Crépel, and N. Guicciardini, eds., pp. 199 207. Elsevier Science, Amsterdam, 2005.

[279] T. Adye, "Unfolding algorithms and tests using RooUnfold", in "Proceedings, PHYSTAT 2011 Workshop on Statistical Issues Related to Discovery Claims in Search Experiments and Unfolding, CERN,Geneva, Switzerland 17-20 January 2011", pp. 313-318, CERN. CERN, Geneva, 2011. arXiv:1105.1160.

[280] A. Hocker and V. Kartvelishvili, "SVD approach to data unfolding", Nucl. Instrum. Meth. A372 (1996) 469-481, arXiv:hep-ph/9509307.

[281] R. Barlow, "Systematic errors: Facts and fictions", in "Conference on Advanced Statistical Techniques in Particle Physics", pp. 134-144. 7 2002. hep-ex/0207026.

[282] C. Shen, J.-F. Paquet, G. S. Denicol, S. Jeon, and C. Gale, "Collectivity and electromagnetic radiation in small systems", Phys. Rev. C 95 (2017), no. 1, 014906, arXiv: 1609.02590. 
[283] M. Gluck, E. Reya, and A. Vogt, "Parton fragmentation into photons beyond the leading order", Phys. Rev. D 48 (1993) 116, [Erratum: Phys.Rev.D 51, 1427 (1995)].

[284] D. G. d'Enterria, "Hard scattering cross-sections at LHC in the Glauber approach: From pp to pA and AA collisions", nucl-ex/0302016. 


\title{
Nicolas Schmidt
}

\section{Curriculum Vitae}

\author{
Personal Information \\ Date of birth 7th April 1992 \\ Place of Starnberg, Germany \\ birth \\ Nationality German \\ Mother Britta Roth \\ Father Gosbert Roth

\section{Education}

Apr. 2017- PhD, Physics, Johann-Wolfgang-Goethe-Universitat, Institut fur Kernphysik, present Frankfurt and Oak Ridge National Laboratory, Oak Ridge, TN.

Planned thesis in experimental particle physics about high momentum light meson measurements and multiplicity dependence of direct photon production.

Oct. 2014- Master of Science, Physics, Ruprecht-Karls-University, Heidelberg.

Apr. 2017 Thesis in experimental particle physics with the title "Neutral meson and direct photon measurements with conversions in ALICE in proton-proton collisions at $\sqrt{s}=0.9,7$ and $8 \mathrm{TeV}^{\prime \prime}$

Oct. 2011- Bachelor of Science, Physics, Ruprecht-Karls-University, Heidelberg.

Oct. 2014 Thesis in experimental particle physics with the title "Neutral Pion Measurements with Conversions in ALICE in pp Collisions at $\sqrt{s}=8 \mathrm{TeV} "$

May 2011 Higher Education Entrance Qualification, Dietrich-Bonhoeffer Gymnasium, Wertheim, Germany.

\section{Publications}

- For the following publications significant contributions have been made:

1. Neutral pion and $\eta$ meson production in proton-proton collisions at $\sqrt{s}=8 \mathrm{TeV}$, ALICE Collaboration, EPJC 78 (2018) 263 (39 citations);

2. Direct photon production at low transverse momentum in proton-proton collisions at $\sqrt{s}=2.76$ and $8 \mathrm{TeV}$, ALICE Collaboration, PRC 99, 024912 (2019) (24 citations)

3. Applicability of transverse mass scaling in hadronic collisions at energies available at the CERN Large Hadron Collider, PRC 96 (2017) 6, 064907 (16 citations)

4. Nuclear modification factor of light neutral mesons up to high transverse momentum in $p-P b$ collisions at $\sqrt{s_{N N}}=8.16 \mathrm{TeV}$, ALICE Collaboration, in preparation

5. Neutral pion and $\eta$ meson production in proton-proton collisions at $\sqrt{s}=5.02 \mathrm{TeV}$, ALICE Collaboration, in preparation 
6. Multiplicity dependence of direct photon production in $p-P b$ collisions at $\sqrt{s_{N N}}=5.02 \mathrm{TeV}$, ALICE Collaboration, in preparation

7. Performance of the ALICE Electromagnetic Calorimeter, ALICE Collaboration, in preparation

- The full list of 129 papers (status 22.10.2020) can be found at: https://inspirehep.net/ search? ln=en\&ln=en\&p=a+N.V.Schmidt .1

- Lead author of 7 internal documents (more information can be provided on request)

\section{Presentations at conferences}

Oct. $2019 \mathbf{1 0}^{\text {th }}$ Hard Probes, Houston, TX, United States.

Oral presentation: Light meson nuclear modification factor in $p-P b$ collisions over an unprecedented $p_{\mathrm{T}}$ range with ALICE, [slides] [proceedings]

Oct. $2018 \mathbf{9}^{\text {th }}$ Hard Probes, Aix-Les-Bains, France.

Oral presentation: Direct photon production and flow at low transverse momenta in $p p$, $p-P b$ and $P b-P b$ collisions, [slides] [proceedings]

Mar. 2018 34 $^{\text {th }}$ Winter Workshop on Nuclear Dynamics, Guadeloupe, France.

Oral presentation: Direct photon production at low transverse momentum in pp collisions at $\sqrt{s}=2.76$ and $8 \mathrm{TeV}$, [slides]

Nov. 20172017 LHC Users Association Meeting, Fermilab.

Flash talk: Neutral pion and $\eta$ meson production in ALICE in proton-proton collisions at $\sqrt{s}=8 \mathrm{TeV}$, [slides]

Mar. 2017 Annual Meeting of the German Physical Society, Devision: Nuclear Physics, Darmstadt, Germany.

Oral presentation: Neutral meson and direct photon measurements with conversions in ALICE in proton-proton collisions at $\sqrt{s}=0.9,7$ and $8 \mathrm{TeV}$

Mar. 2016 Annual Meeting of the German Physical Society, Devision: Nuclear Physics, Darmstadt, Germany.

Oral presentation: Neutral Meson Measurements using Conversions in Proton-Proton Collisions at $\sqrt{s}=8 \mathrm{TeV}$ in ALICE 


\section{Acknowledgements}

I'd like to express my gratitude to Prof. Dr. Henner Büsching and my supervisor Dr. Constantinos Loizides for giving me the opportunity to carry out my doctoral studies as part of their working group and institutes. There was always an open ear for any matter as well as continuous support for my growth in the scientific environment.

Further appreciation goes towards Dr. Friederike Bock, who i value as a friend and colleague alike. She open-heartedly and persistently kept me focused on my work and provided valuable discussions and inputs to further advance my studies.

I would also like to thank all members of the ORNL working group for their fruitful discussions and persistent interest in my work. Special thanks go to Martin Poghosyan and Alex Rusu, who worked tirelessly on improving our understanding of the EMCal detector, which allowed for the presented measurements in this thesis to extend into previously unexplored regions.

I'd also like to thank my office-mate Florian Jonas for his open ear and occasional distractions from my work, which created an enjoyable work environment.

I'm thankful for my whole family and their never-ending support during my time at CERN. Our frequent communication made the spatial distance between us less significant.

Lastly, i'd like to thank Mike Sas for his cooperative efforts in multiple analyses and for proofreading my thesis alongside others. 